\title{
The Anisotropic Effect of Thiocarbonyl and Selenocarbonyl Functional Groups and the Interplay of \\ Thio(seleno)amide/Vinylogous Thio(seleno)amide "Resonance"
}

\author{
Erich Kleinpeter, ${ }^{\dagger} *$ Anja Schulenburg, ${ }^{\dagger}$ Ines $\mathrm{Zug}^{\ddagger}$ and Horst Hartmann ${ }^{\ddagger}$ \\ ${ }^{\dagger}$ Universität Potsdam, Chemisches Institut, P.O. Box 6015 53, D-14415 Potsdam, Germany \\ ${ }^{\ddagger}$ Fachhochschule Merseburg, Fachbereich Chemie, Geusaer Str., D-06217 Merseburg, Germany \\ kp@chem.uni-potsdam.de
}

\section{Supporting Information}

Table of Contents:

General procedures for the synthesis of $1-4$.

Table S1. ${ }^{1} \mathrm{H}$ NMR chemical shifts and ${ }^{3} J_{\mathrm{H}, \mathrm{H}}$ coupling constants for compounds $1-4$

$(\delta / \mathrm{ppm}, J / \mathrm{Hz}), \mathrm{R}_{2} \mathrm{~N}-\mathrm{C}_{1} \mathrm{H}=\mathrm{X}_{2}-\mathrm{C}_{3}(\mathrm{~S} / \mathrm{Se})-\mathrm{NR}_{2}^{\prime}(\mathrm{X}=\mathrm{CH}, \mathrm{N})$.

Table S2. ${ }^{13} \mathrm{C}$ NMR chemical shifts for compounds $1-4(\delta / \mathrm{ppm})$,

$\mathrm{R}_{2} \mathrm{~N}-\mathrm{C}_{1} \mathrm{H}=\mathrm{X}_{2}-\mathrm{C}_{3}(\mathrm{~S} / \mathrm{Se})-\mathrm{NR}_{2}^{\prime}(\mathrm{X}=\mathrm{CH}, \mathrm{N})$.

Table S3. Ground state occupation numbers of various orbitals by NBO analysis of the amino-substituted thio(seleno)acrylamides $1-4$ as calculated at the HF/6-31G* level of theory.

Table S4. Energies of both the GS and TS of the restricted rotation about the $\mathrm{C}_{1}-\mathrm{N}$ and $\mathrm{C}_{3}-\mathrm{N}$ partial double bonds and the corresponding barriers to rotation for $1 \mathrm{~b}$ and $3 \mathrm{~b} \ldots \ldots . . \mathrm{S} 9$

Table S5. Ground state bond lengths of amino-substituted thio(seleno)acrylamides 1-4 as calculated at the $\mathrm{HF} / 6-31 \mathrm{G}^{*}$ level of theory.

Table S6. Barriers to rotation of compounds 1-4 as theoretically calculated at the HF/6$31 \mathrm{G}^{*}$ and $\mathrm{B} 3 \mathrm{LYP} / 6-31 \mathrm{G}^{*}$ levels of theory.....

Table S7. Calculated ground state energies and transition state energies for the restricted rotations about the $\mathrm{C}_{1}-\mathrm{N}$ and $\mathrm{C}_{3}-\mathrm{N}$ partial double bonds at various levels of theory

Figure S1. The four isomers of the amino-substituted thioacrylamide $\mathbf{3 b}$ and their 
relative energies.

Figure S2. The conformers resulting from the restricted rotation about the $\mathrm{C}^{1}-\mathrm{N}$ partial double bond and their relative energies. S19

Figure S3. The conformers resulting from the restricted rotation about the $\mathrm{C}^{3}, \mathrm{~N}$ partial

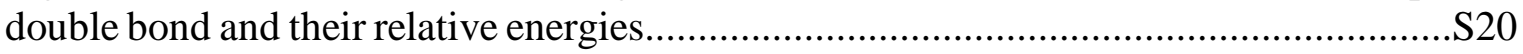

Figure S4. The calculated global minimum structures of $1 \mathrm{e}, 1 \mathrm{~g}$, and 2......................S21

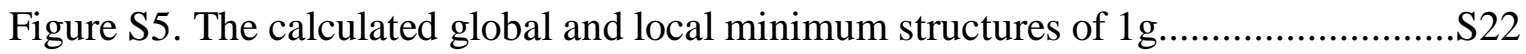

Figure S6. Correlation of experimental and theoretically calculated barriers to restricted rotations about $\mathrm{C}^{1}-\mathrm{N}$ and $\mathrm{C}^{3}-\mathrm{N}$ partial double bonds on the SCIPCM-HF/6-31G* and B3LYP/6-31G* levels of theory of amino-substituted thia(seleno)acrylamides 1-4.....S24

Table S8. Coordinates and absolute energies of substituted 1,3-diheterocyclohexanes (cf. Scheme 1) at HF/6-31G*-level of theory. 


\section{General procedures for the synthesis of 1-4}

Preparation of 3-amino-thioacrylamides 1 (general procedure according to reference 44 and Scheme 11)

A solution of a $N, N^{\prime}$-persubstituted 1-chlorovinamidinium salt 6 ( $\left.0.1 \mathrm{~mol}\right)$ in acetonitrile (100 $\mathrm{mL}$ ) was added with stirring at r.t. to an aqueous solution $(100 \mathrm{~mL})$ of sodium sulphide $(0.1 \mathrm{~mol}$, $7.8 \mathrm{~g}$ ). The products of the general structure $\mathbf{1}$ formed crystallized and were isolated by filtration and purified by recrystallisation.

Preparation of 3-amino-2-aza-thioacrylamides 3 (general procedures)

Method A: A solution of a $N, N$ '-persubstituted 1-chloro-2-azavinamidinium salt 8 ( $0.1 \mathrm{~mol})$, prepared according to ref. 48 , in acetonitrile $(100 \mathrm{~mL})$ was added with stirring at r.t. to an aqueous solution $(100 \mathrm{~mL})$ of sodium sulphide $(0.1 \mathrm{~mol}, 7.8 \mathrm{~g})$. The products formed crystallized and were isolated by filtration and purified by recrystallisation.

Method B: A mixture of $N, N$-disubstituted thiourea $9(20 \mathrm{mmol})$, triethyl orthoformiate $(4.5 \mathrm{~g}, 30$ $\mathrm{mmol}$ ), and morpholine $(2.6 \mathrm{~g}, 30 \mathrm{mmol})$ was heated at $120^{\circ} \mathrm{C}$ under removal of ethanol formed. After cooling to r.t., the solid products were filtered off and recrystallized.

Preparation of 3-amino-2-aza-selenoacrylamides 4 (general procedures)

Method A: In analogy to method A, previously described, a solution of a $N, N$ '-persubstituted 1chloro-2-azavinamidinium salt $8(0.1 \mathrm{~mol})$ in acetonitrile $(100 \mathrm{~mL})$ was added with stirring at r.t. to an ethanolic solution of sodium selenide, freshly prepared by addition of $\mathrm{NaBH}_{4}(0.2 \mathrm{~mol}, 7.6$ $\mathrm{g})$ to a stirred solution of elemental selenium $(0.1 \mathrm{~mol}, 7.9 \mathrm{~g})$ in ethanol $(100 \mathrm{~mL})$. The products formed crystallized and were isolated by filtration and purified by recrystallisation.

Method A1: In analogy to the method A to an ethanolic solution of sodium selenide, freshly prepared by addition of $\mathrm{NaBH}_{4}(0.1 \mathrm{~mol}, 3.8 \mathrm{~g})$ to a stirred solution of elemental selenium $(0.05$ mol, $4.0 \mathrm{~g})$ in ethanol $(100 \mathrm{~mL})$, an ethanolic solution $(50 \mathrm{~mL})$ of a 1-methylmercapto-2azavinamidinium iodide, prepared by reaction of a $N, N^{\prime}$-persubstituted 3-amino-2azathioacrylamide $3(0.05 \mathrm{~mol})$ with methyl iodide $(0.05 \mathrm{~mol}, 7.2 \mathrm{~g})$ was added with stirring at r.t.. The products formed crystallized and were isolated by filtration and purified by recrystallisation.

Method B: In analogy to method B, previously described, a mixture of $N, N$-disubstituted selenourea $10(20 \mathrm{mmol})$, triethyl orthoformiate $(4.5 \mathrm{~g}, 30 \mathrm{mmol})$, and morpholine $(2.6 \mathrm{~g}, 30$ mmol) was heated at $120{ }^{\circ} \mathrm{C}$ under removal of ethanol formed. After cooling to r.t., the solid products were filtered off and recrystallized. 
Table 5. Characteristic data of compounds 1 - 4 prepared

\begin{tabular}{|c|c|c|c|c|c|c|c|c|c|c|c|c|}
\hline KPCode & $X$ & $\mathrm{Y}$ & $\mathrm{R}_{2} \mathrm{~N}$ & $\mathrm{R}_{2} \mathrm{~N}^{\prime}$ & $\begin{array}{l}\text { Yield } \\
\text { [\%] }\end{array}$ & Method & $\begin{array}{l}\text { m.p. } \\
{\left[{ }^{\circ} \mathrm{C}\right]}\end{array}$ & $\begin{array}{l}\text { Formula } \\
\text { calcd. } \\
\text { (m.w.) } \\
\text { found }\end{array}$ & C & $\mathrm{H}$ & $\mathrm{N}$ & $\mathrm{S}$ \\
\hline $1 \mathrm{a}$ & $\mathrm{S}$ & $\mathrm{CH}$ & Pip & $\mathrm{Me}_{2} \mathrm{~N}$ & 61 & A & 98-101 & $\begin{array}{l}\mathrm{C}_{10} \mathrm{H}_{18} \mathrm{~N}_{2} \mathrm{~S} \\
(198.33)\end{array}$ & $\begin{array}{l}60.56 \\
59.71\end{array}$ & $\begin{array}{l}9.15 \\
9.14\end{array}$ & $\begin{array}{l}14.12 \\
14.10\end{array}$ & $\begin{array}{l}16.17 \\
15.98\end{array}$ \\
\hline $1 \mathrm{~b}$ & $\mathrm{~S}$ & $\mathrm{CH}$ & $\mathrm{NMe}_{2}$ & $\mathrm{Me}_{2} \mathrm{~N}$ & 88 & A & $\begin{array}{l}116- \\
120\end{array}$ & $\mathrm{C}_{7} \mathrm{H}_{14} \mathrm{~N}_{2} \mathrm{~S}$ & 53.12 & 8.92 & 17.70 & 20.26 \\
\hline $1 \mathrm{c}$ & $\mathrm{S}$ & $\mathrm{CH}$ & Morph & $\mathrm{Me}_{2} \mathrm{~N}$ & 71 & A & $\begin{array}{c}132- \\
134\end{array}$ & $\begin{array}{l}(158.27) \\
\mathrm{C}_{9} \mathrm{H}_{16} \mathrm{~N}_{2} \mathrm{OS}\end{array}$ & $\begin{array}{l}53.19 \\
53.97\end{array}$ & $\begin{array}{l}8.90 \\
8.05\end{array}$ & $\begin{array}{l}17.55 \\
13.99\end{array}$ & $\begin{array}{l}20.26 \\
16.01\end{array}$ \\
\hline 1d & $\mathrm{S}$ & $\mathrm{CH}$ & Pyrr & $\mathrm{Me}_{2} \mathrm{~N}$ & 94 & A & $\begin{array}{l}104- \\
107\end{array}$ & $\begin{array}{l}(200.30) \\
\mathrm{C}_{9} \mathrm{H}_{16} \mathrm{~N}_{2} \mathrm{~S}\end{array}$ & $\begin{array}{l}53.99 \\
58.65\end{array}$ & $\begin{array}{l}7.96 \\
8.75\end{array}$ & $\begin{array}{l}13.78 \\
15.20\end{array}$ & $\begin{array}{l}16.17 \\
17.40\end{array}$ \\
\hline $1 e$ & $\mathrm{~S}$ & $\mathrm{CH}$ & $\mathrm{NPh}_{2}$ & $\mathrm{Me}_{2} \mathrm{~N}$ & 73 & A & $\begin{array}{l}210- \\
211\end{array}$ & $\begin{array}{l}(184.30) \\
\mathrm{C}_{17} \mathrm{H}_{18} \mathrm{~N}_{2} \mathrm{~S}\end{array}$ & $\begin{array}{l}57.60 \\
72.30\end{array}$ & $\begin{array}{l}8.60 \\
6.42\end{array}$ & $\begin{array}{c}15.19 \\
9.92\end{array}$ & $\begin{array}{l}17.21 \\
11.35\end{array}$ \\
\hline 1f & $\mathrm{S}$ & $\mathrm{CH}$ & $\mathrm{NMePh}$ & $\mathrm{Me}_{2} \mathrm{~N}$ & 88 & A & $61-63$ & $\begin{array}{l}(282.40) \\
\mathrm{C}_{12} \mathrm{H}_{16} \mathrm{~N}_{2} \mathrm{~S} \\
(220.33)\end{array}$ & $\begin{array}{l}72.42 \\
65.41 \\
65.37\end{array}$ & $\begin{array}{l}6.39 \\
7.32 \\
7.34\end{array}$ & $\begin{array}{c}9.66 \\
12.71 \\
12.62\end{array}$ & $\begin{array}{l}11.74 \\
14.55 \\
14.51\end{array}$ \\
\hline $1 \mathrm{~g}$ & $\mathrm{~S}$ & $\mathrm{CH}$ & $\begin{array}{l}\text { NPh- } \\
\text { 1Naph }\end{array}$ & $\mathrm{Me}_{2} \mathrm{~N}$ & 62 & A & $\begin{array}{l}228- \\
231\end{array}$ & $\mathrm{C}_{21} \mathrm{H}_{20} \mathrm{~N}_{2} \mathrm{~S}$ & 75.87 & 6.06 & 8.43 & 9.64 \\
\hline 2 & $\mathrm{Se}$ & $\mathrm{CH}$ & Phenoth & $\mathrm{Me}_{2} \mathrm{~N}$ & 75 & A & $\begin{array}{c}156- \\
159\end{array}$ & $\begin{array}{l}(332.46) \\
\mathrm{C}_{17} \mathrm{H}_{16} \mathrm{~N}_{2} \mathrm{~S}_{2}\end{array}$ & $\begin{array}{l}75.55 \\
65.35\end{array}$ & $\begin{array}{l}6.03 \\
5.16\end{array}$ & $\begin{array}{l}8.50 \\
8.97\end{array}$ & $\begin{array}{c}9.24 \\
20.53\end{array}$ \\
\hline 3b & $S$ & $\mathrm{~N}$ & $\mathrm{Me}_{2} \mathrm{~N}$ & $\mathrm{Me}_{2} \mathrm{~N}$ & 70 & A & $92-93$ & $\begin{array}{l}(312.45) \\
\mathrm{C}_{6} \mathrm{H}_{13} \mathrm{~N}_{3} \mathrm{~S} \\
(159.25)\end{array}$ & $\begin{array}{l}65.44 \\
45.25 \\
45.33\end{array}$ & $\begin{array}{l}5.20 \\
8.23 \\
8.07\end{array}$ & $\begin{array}{c}8.88 \\
26.29 \\
26.22\end{array}$ & $\begin{array}{l}20.34 \\
20.13 \\
20.22\end{array}$ \\
\hline $3 c$ & $\mathrm{~S}$ & $\mathrm{~N}$ & Morph & $\mathrm{Me}_{2} \mathrm{~N}$ & 45 & A & $78-80$ & $\begin{array}{l}\mathrm{C}_{8} \mathrm{H}_{15} \mathrm{~N}_{3} \mathrm{OS} \\
(201.29)\end{array}$ & $\begin{array}{l}47.73 \\
47.77\end{array}$ & $\begin{array}{l}7.51 \\
7.06\end{array}$ & $\begin{array}{l}20.88 \\
20.52\end{array}$ & $\begin{array}{l}15.93 \\
15.83\end{array}$ \\
\hline 3d & S & $\mathrm{N}$ & Pyrr & $\mathrm{Me}_{2} \mathrm{~N}$ & 55 & A & $74-76$ & $\begin{array}{l}\mathrm{C}_{8} \mathrm{H}_{15} \mathrm{~N}_{3} \mathrm{~S} \\
(185.29)\end{array}$ & $\begin{array}{l}51.86 \\
51.92\end{array}$ & $\begin{array}{l}8.16 \\
8.05\end{array}$ & $\begin{array}{l}22.68 \\
22.44\end{array}$ & $\begin{array}{l}17.31 \\
17.46\end{array}$ \\
\hline $3 h$ & $\mathrm{~S}$ & $\mathrm{~N}$ & $\mathrm{Me}_{2} \mathrm{~N}$ & $\mathrm{NMePh}$ & 60 & A & $56-58$ & $\begin{array}{l}\mathrm{C}_{11} \mathrm{H}_{15} \mathrm{~N}_{3} \mathrm{~S} \\
(221.32)\end{array}$ & $\begin{array}{l}59.69 \\
59.77\end{array}$ & $\begin{array}{l}6.83 \\
6.51\end{array}$ & $\begin{array}{l}18.99 \\
18.67\end{array}$ & $\begin{array}{l}14.49 \\
14.34\end{array}$ \\
\hline $3 \mathbf{i}$ & S & $\mathrm{N}$ & $\mathrm{Me}_{2} \mathrm{~N}$ & Morph & 72 & A & $94-96$ & $\begin{array}{l}\mathrm{C}_{8} \mathrm{H}_{15} \mathrm{~N}_{3} \mathrm{OS} \\
(201.29)\end{array}$ & $\begin{array}{l}47.73 \\
47.55\end{array}$ & $\begin{array}{l}7.51 \\
7.10\end{array}$ & $\begin{array}{l}20.88 \\
20.25\end{array}$ & $\begin{array}{l}15.93 \\
15.80\end{array}$ \\
\hline $3 k$ & $\mathrm{~S}$ & $\mathrm{~N}$ & Morph & $\mathrm{NMePh}$ & 32 & A & $\begin{array}{l}110- \\
110\end{array}$ & $\mathrm{C}_{13} \mathrm{H}_{17} \mathrm{~N}_{3} \mathrm{OS}$ & 59.29 & 6.51 & 15.56 & 12.18 \\
\hline 31 & S & $\mathrm{N}$ & Pyrr & Morph & 35 & A & $\begin{array}{l}122- \\
124\end{array}$ & $\begin{array}{l}(263.36) \\
\mathrm{C}_{13} \mathrm{H}_{17} \mathrm{~N}_{3} \mathrm{OS}\end{array}$ & $\begin{array}{l}59.28 \\
52.83\end{array}$ & $\begin{array}{l}6.47 \\
7.54\end{array}$ & $\begin{array}{l}15.48 \\
18.48\end{array}$ & $\begin{array}{l}11.10 \\
14.11\end{array}$ \\
\hline $3 m$ & S & $\mathrm{N}$ & Pyrr & $\mathrm{NMePh}$ & 82 & A & $\begin{array}{l}135- \\
136\end{array}$ & $\begin{array}{l}(227.33) \\
\mathrm{C}_{13} \mathrm{H}_{17} \mathrm{~N}_{3} \mathrm{~S}\end{array}$ & $\begin{array}{l}52.99 \\
63.12\end{array}$ & $\begin{array}{l}7.49 \\
6.93\end{array}$ & $\begin{array}{l}18.27 \\
16.99\end{array}$ & $\begin{array}{l}14.07 \\
12.96\end{array}$ \\
\hline $3 n$ & $\mathrm{~S}$ & $\mathrm{~N}$ & $\mathrm{NPh}_{2}$ & Morph & 93 & B & $\begin{array}{l}242- \\
244\end{array}$ & $\begin{array}{l}(247.36) \\
\mathrm{C}_{18} \mathrm{H}_{19} \mathrm{~N}_{3} \mathrm{OS}\end{array}$ & $\begin{array}{l}62.60 \\
66.43\end{array}$ & $\begin{array}{l}6.65 \\
5.88\end{array}$ & $\begin{array}{l}16.66 \\
12.91\end{array}$ & $\begin{array}{l}12.90 \\
9.85\end{array}$ \\
\hline 30 & S & $\mathrm{N}$ & $\mathrm{NMePh}$ & Morph & 88 & B & $98-100$ & $\begin{array}{l}(325.44) \\
\mathrm{C}_{13} \mathrm{H}_{17} \mathrm{~N}_{3} \mathrm{OS} \\
(263.36)\end{array}$ & $\begin{array}{l}66.30 \\
59.29 \\
59.62 \\
\end{array}$ & $\begin{array}{l}5.99 \\
6.51 \\
6.54\end{array}$ & $\begin{array}{l}12.54 \\
15.56 \\
15.71 \\
\end{array}$ & $\begin{array}{l}10.12 \\
12.18 \\
12.57\end{array}$ \\
\hline
\end{tabular}


Table 5. Continued

\begin{tabular}{|c|c|c|c|c|c|c|c|c|c|c|c|c|}
\hline KPCode & $\mathrm{X}$ & $\mathrm{Y}$ & $\mathrm{R}_{2} \mathrm{~N}$ & $\mathrm{R}_{2} \mathrm{~N}^{\prime}$ & $\begin{array}{l}\text { Yield } \\
{[\%]}\end{array}$ & Method & $\begin{array}{l}\text { m.p. } \\
{\left[{ }^{\circ} \mathrm{C}\right]}\end{array}$ & $\begin{array}{l}\text { Formula } \\
\text { (m.w.) }\end{array}$ & $\mathrm{C}$ & $\mathrm{H}$ & $\mathrm{N}$ & $\mathrm{S}$ \\
\hline \multirow[t]{2}{*}{$3 \mathbf{p}$} & $\mathrm{S}$ & $\mathrm{N}$ & Morph & Morph & 50 & B & $156-158$ & $\mathrm{C}_{10} \mathrm{H}_{17} \mathrm{~N}_{3} \mathrm{O}_{2} \mathrm{~S}$ & 49.36 & 7.04 & 17.24 & 13.18 \\
\hline & & & & & & & & $(243.33)$ & 49.31 & 6.91 & 17.14 & 13.25 \\
\hline \multirow[t]{2}{*}{$4 b$} & $\mathrm{Se}$ & $\mathrm{N}$ & $\mathrm{Me}_{2} \mathrm{~N}$ & $\mathrm{Me}_{2} \mathrm{~N}$ & 55 & A & $106-108$ & $\mathrm{C}_{6} \mathrm{H}_{13} \mathrm{~N}_{3} \mathrm{Se}$ & 34.96 & 6.36 & 20.38 & \\
\hline & & & & & & & & $(206.15)$ & 35.13 & 5.95 & 20.02 & \\
\hline \multirow[t]{2}{*}{$4 c$} & $\mathrm{Se}$ & $\mathrm{N}$ & Morph & $\mathrm{Me}_{2} \mathrm{~N}$ & 25 & A & $65-67$ & $\mathrm{C}_{6} \mathrm{H}_{13} \mathrm{~N}_{3} \mathrm{Se}$ & 38.72 & 6.09 & 16.93 & \\
\hline & & & & & & & & (248.18) & 38.81 & 5.69 & 16.59 & \\
\hline \multirow[t]{2}{*}{$4 d$} & $\mathrm{Se}$ & $\mathrm{N}$ & Pyrr & $\mathrm{Me}_{2} \mathrm{~N}$ & 58 & A & $90-92$ & $\mathrm{C}_{8} \mathrm{H}_{15} \mathrm{~N}_{3} \mathrm{Se}$ & 41.38 & 6.51 & 18.10 & \\
\hline & & & & & & & & (232.19) & 41.38 & 6.35 & 17.68 & \\
\hline \multirow[t]{2}{*}{$4 e$} & $\mathrm{Se}$ & $\mathrm{N}$ & $\mathrm{NPh}_{2}$ & $\mathrm{Me}_{2} \mathrm{~N}$ & 10 & A1 & $212-213$ & $\mathrm{C}_{16} \mathrm{H}_{17} \mathrm{~N}_{3} \mathrm{Se}$ & 58.18 & 5.19 & 12.72 & \\
\hline & & & & & & & & $(330.29)$ & 58.13 & 5.60 & 12.46 & \\
\hline \multirow[t]{2}{*}{$4 f$} & $\mathrm{Se}$ & $\mathrm{N}$ & $\mathrm{NMePh}$ & $\mathrm{Me}_{2} \mathrm{~N}$ & 48 & A1 & $156-158$ & $\mathrm{C}_{11} \mathrm{H}_{15} \mathrm{~N}_{3} \mathrm{Se}$ & 49.26 & 5.64 & 15.67 & \\
\hline & & & & & & & & $(268.22)$ & 49.45 & 5.33 & 15.65 & \\
\hline \multirow[t]{2}{*}{$4 i$} & $\mathrm{Se}$ & $\mathrm{N}$ & $\mathrm{Me}_{2} \mathrm{~N}$ & Morph & 50 & A & $98-100$ & $\mathrm{C}_{8} \mathrm{H}_{15} \mathrm{~N}_{3} \mathrm{OSe}$ & 38.72 & 6.09 & 16.93 & \\
\hline & & & & & & & & (248.18) & 38.72 & 5.66 & 16.53 & \\
\hline \multirow[t]{2}{*}{41} & $\mathrm{Se}$ & $\mathrm{N}$ & Pyrr & Morph & 44 & A & $126-128$ & $\mathrm{C}_{10} \mathrm{H}_{17} \mathrm{~N}_{3} \mathrm{OSe}$ & 43.80 & 6.25 & 15.32 & \\
\hline & & & & & & & & (274.22) & 43.86 & 6.14 & 14.88 & \\
\hline \multirow[t]{2}{*}{$4 m$} & $\mathrm{Se}$ & $\mathrm{N}$ & Pyrr & $\mathrm{NMePh}$ & 60 & A & $127-129$ & $\mathrm{C}_{13} \mathrm{H}_{17} \mathrm{~N}_{3} \mathrm{Se}$ & 53.06 & 5.82 & 14.28 & \\
\hline & & & & & & & & $(294.26)$ & 52.98 & 5.75 & 14.21 & \\
\hline \multirow[t]{2}{*}{$4 p$} & $\mathrm{Se}$ & $\mathrm{N}$ & Morph & Morph & 45 & A1 & $155-157$ & $\mathrm{C}_{10} \mathrm{H}_{17} \mathrm{~N}_{3} \mathrm{O}_{2} \mathrm{Se}$ & 41.38 & 5.90 & 14.48 & \\
\hline & & & & & & & & $(290.22)$ & 41.42 & 5.77 & 14.23 & \\
\hline
\end{tabular}




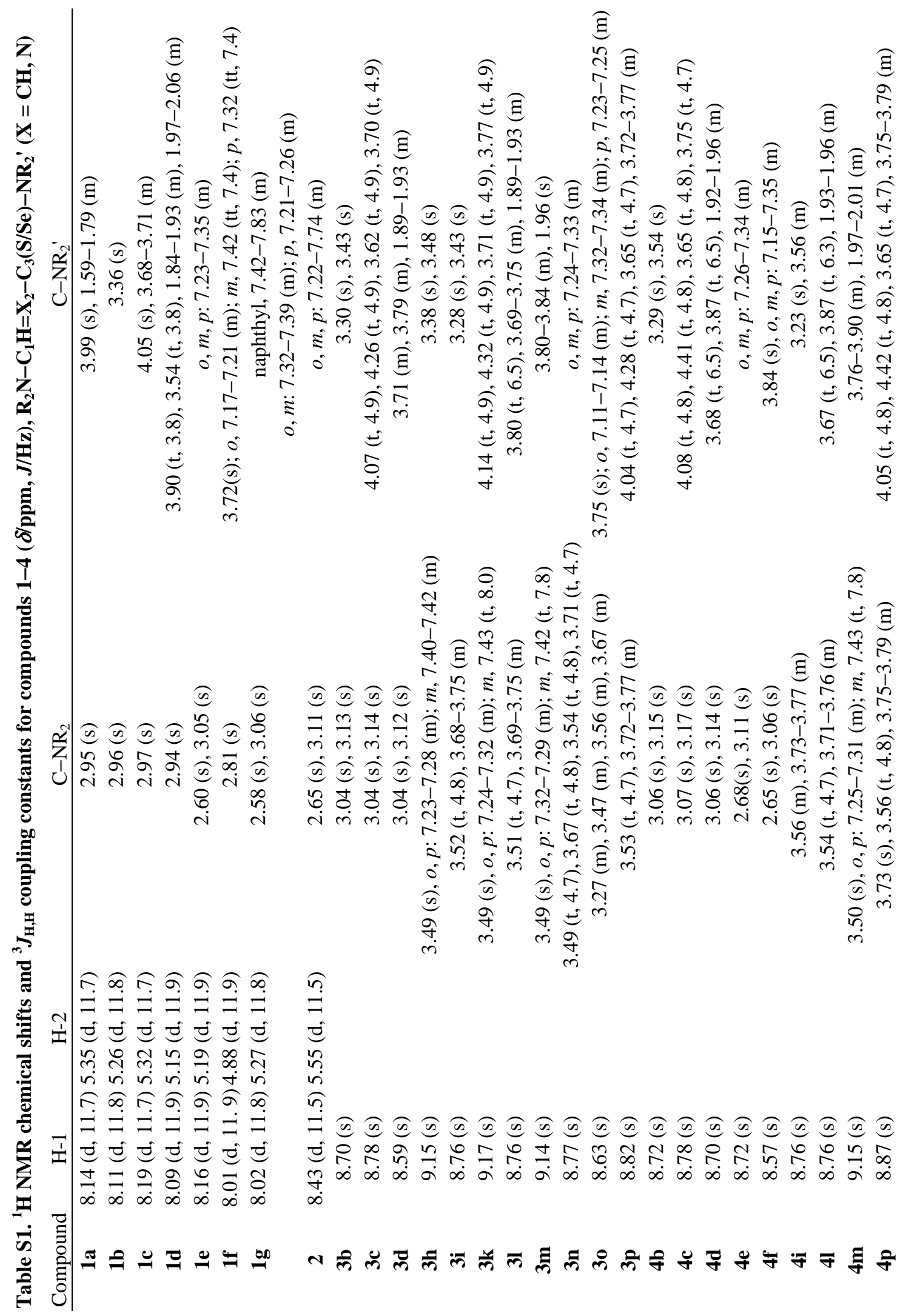


is

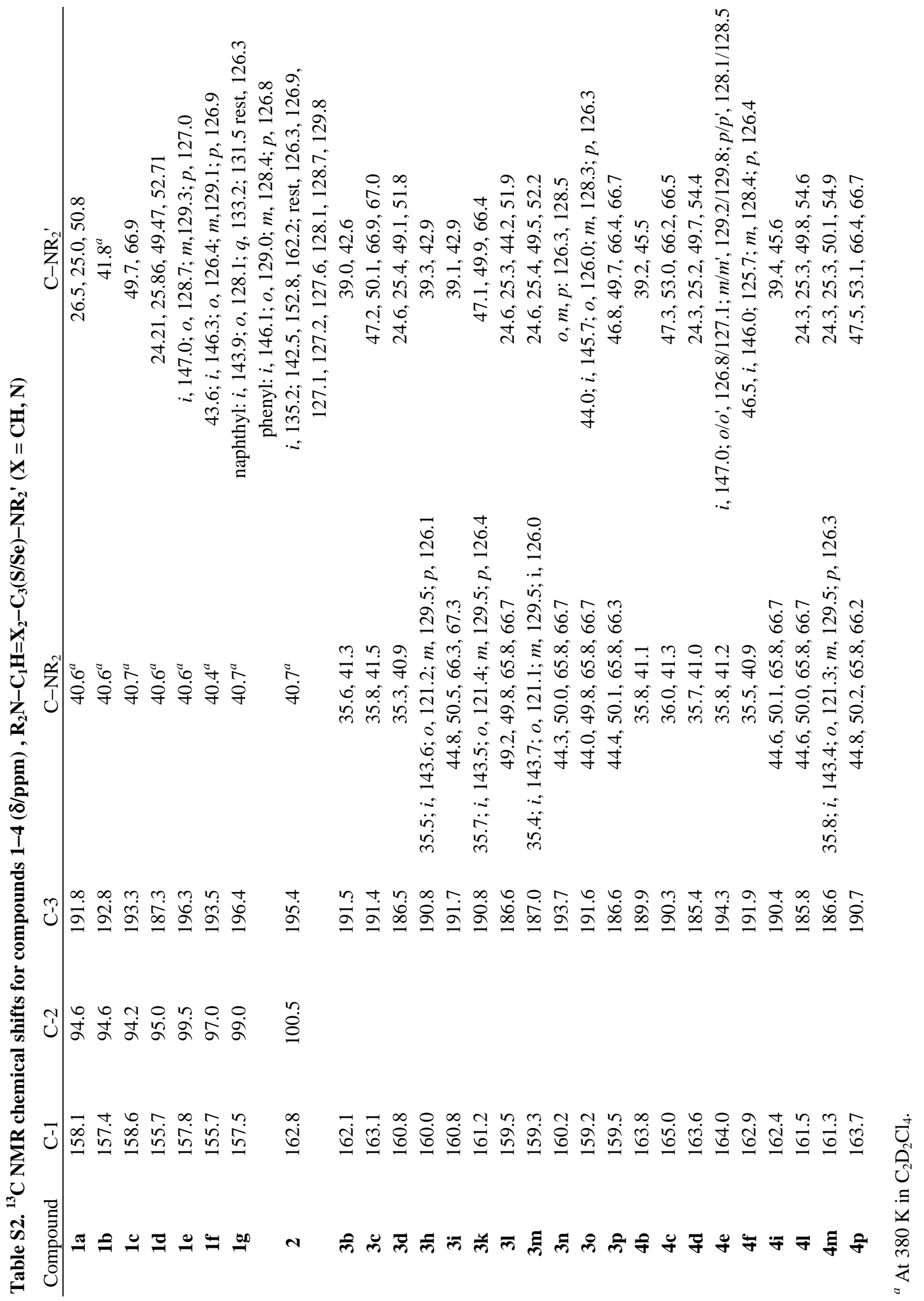




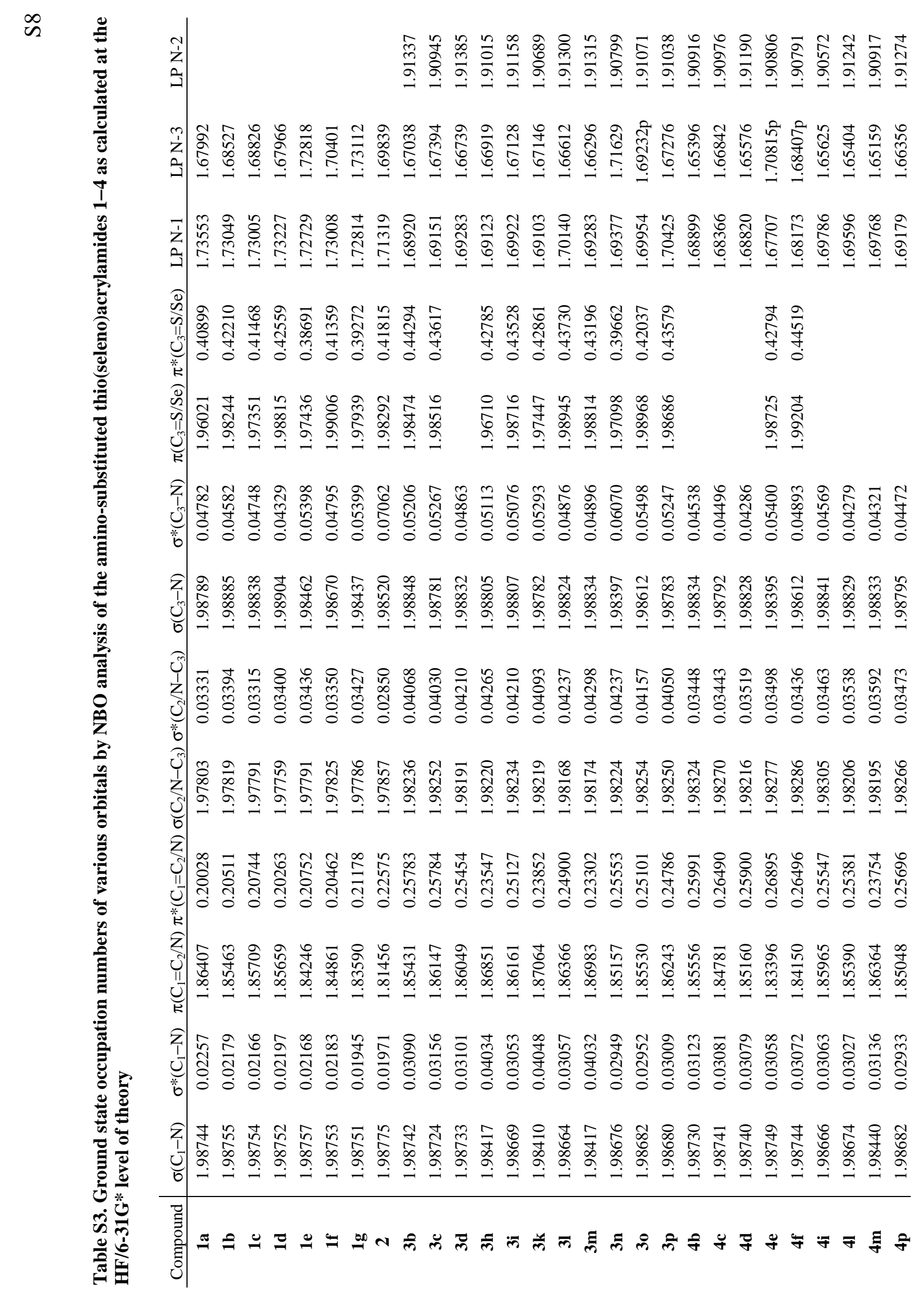




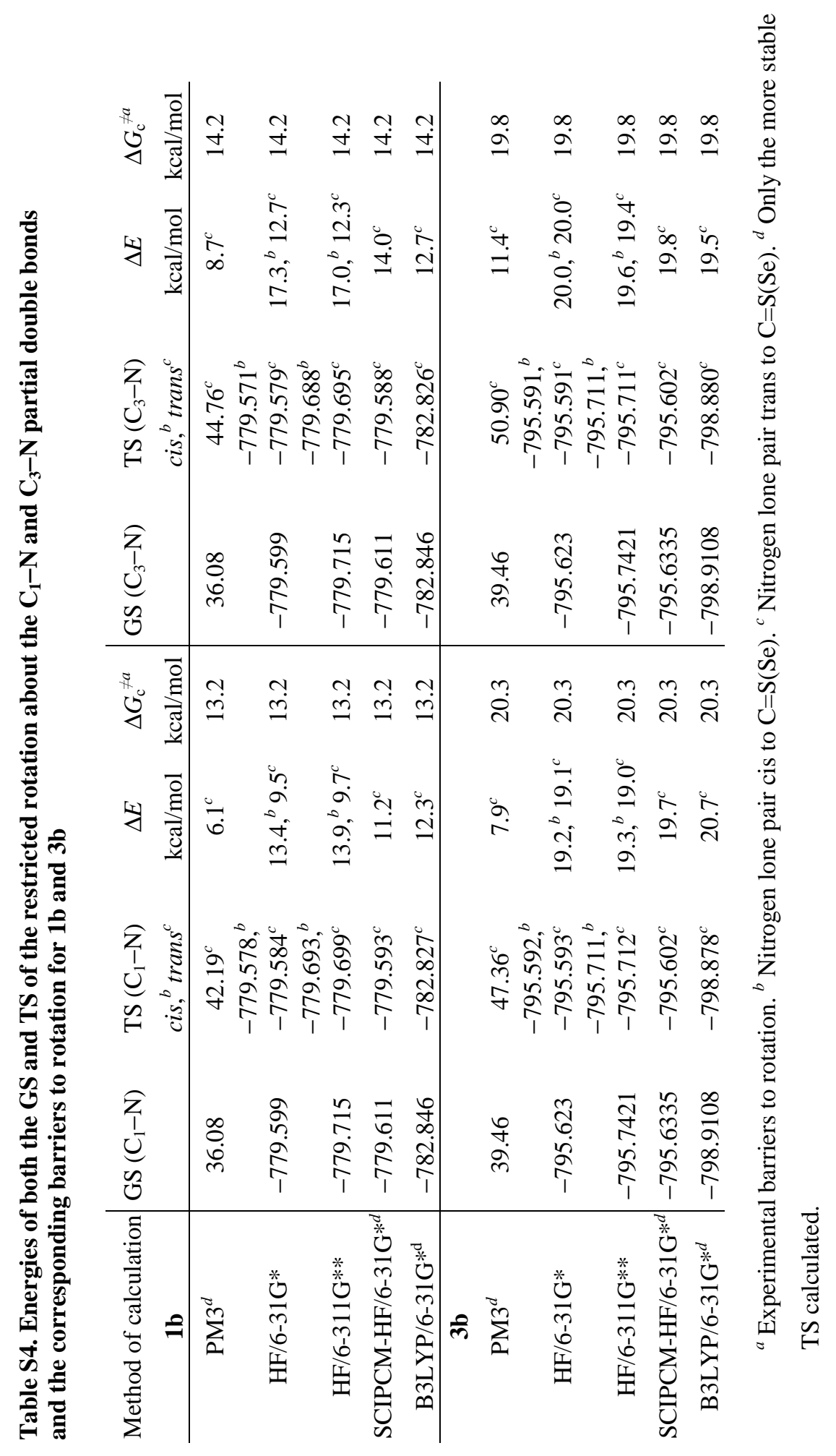


$\frac{0}{n}$

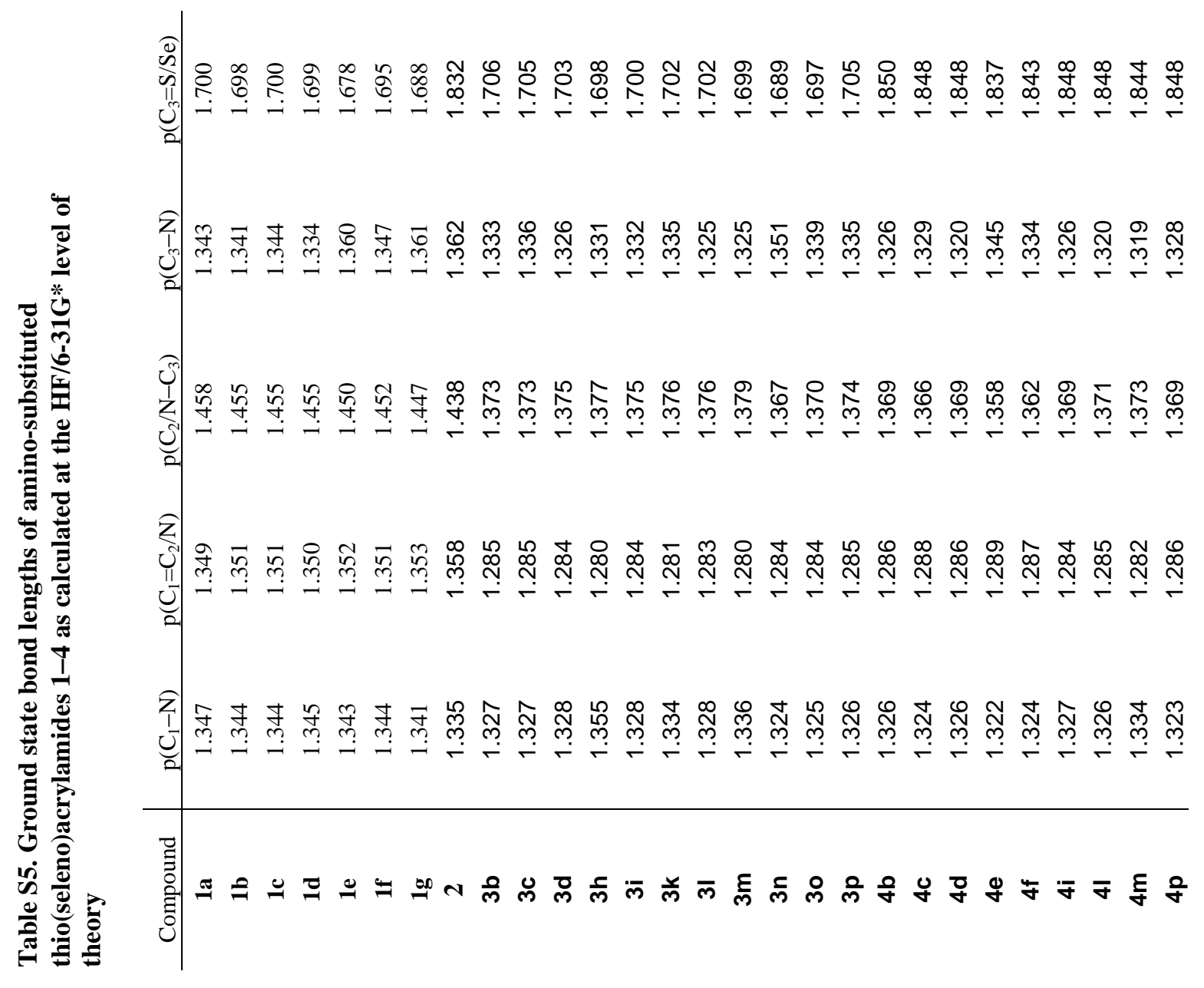




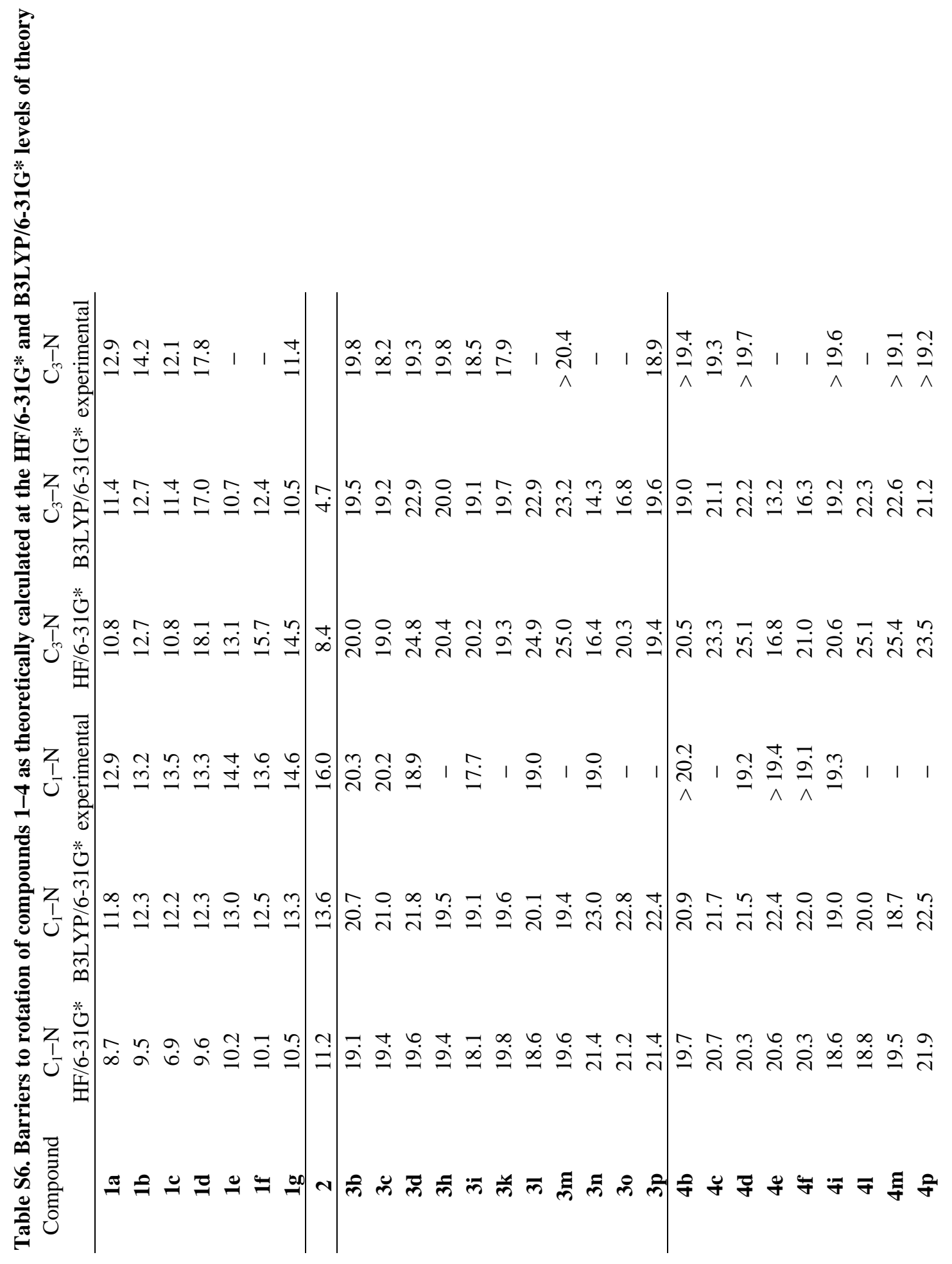


के

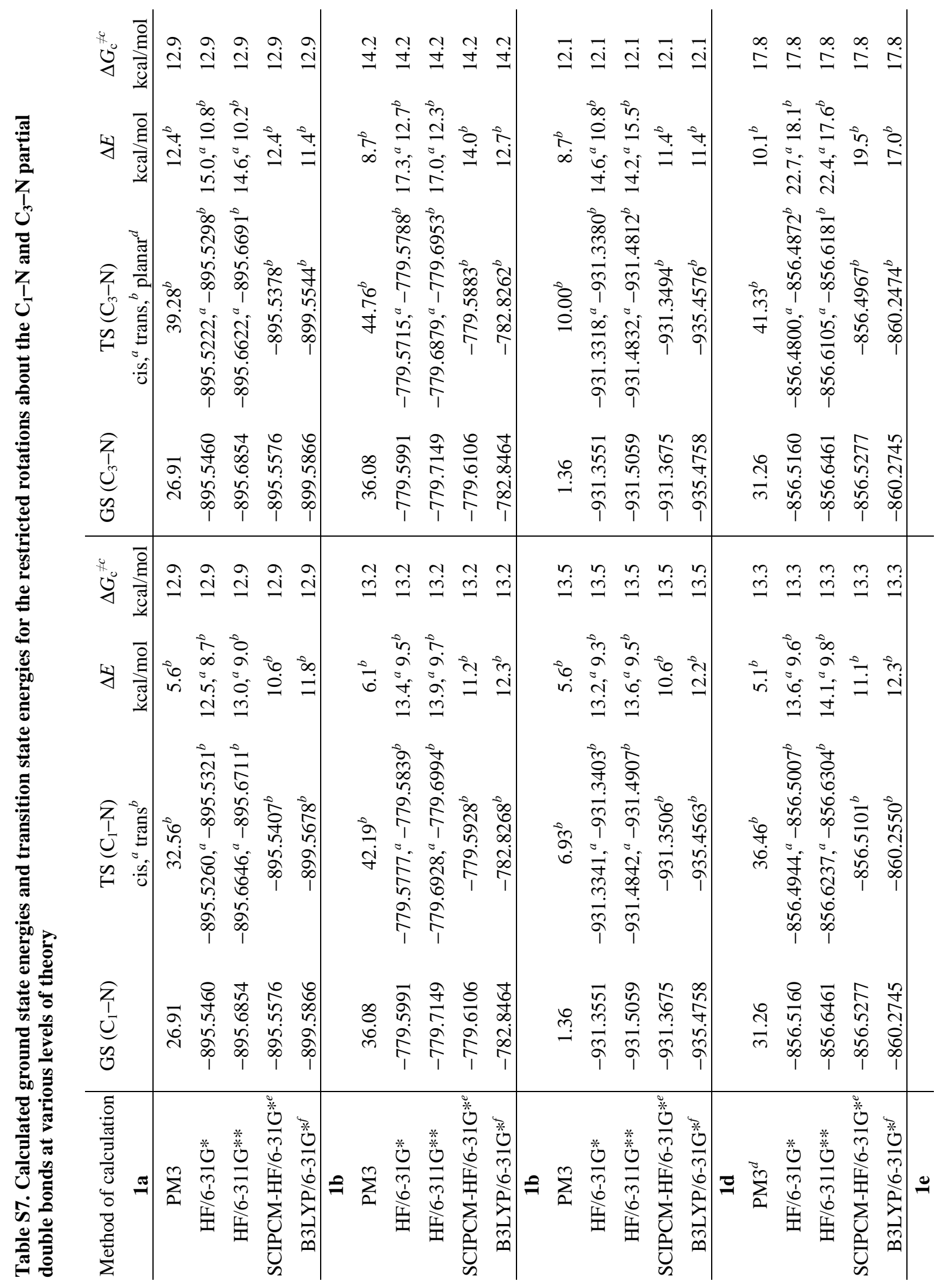


$\frac{m}{n}$

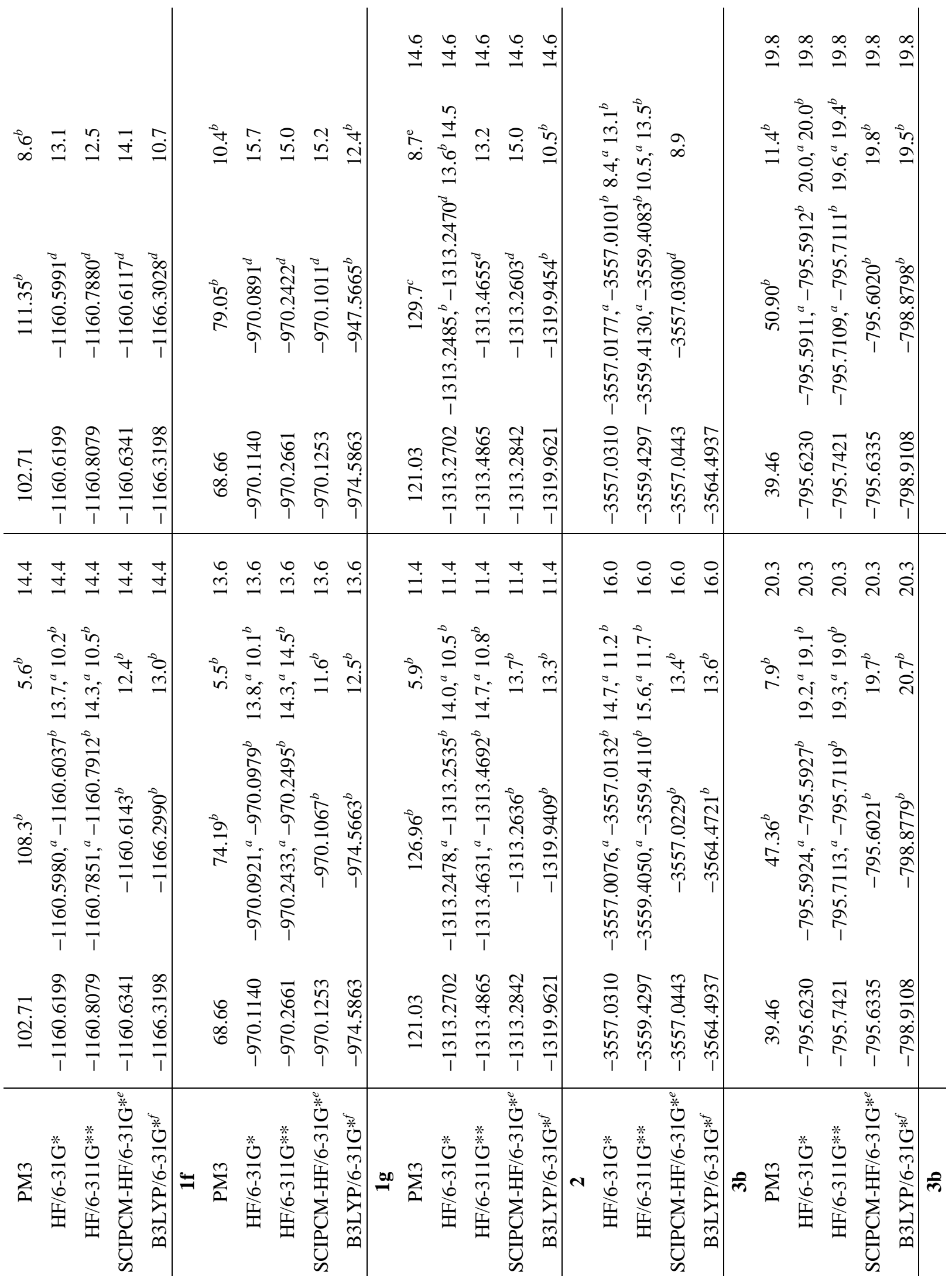




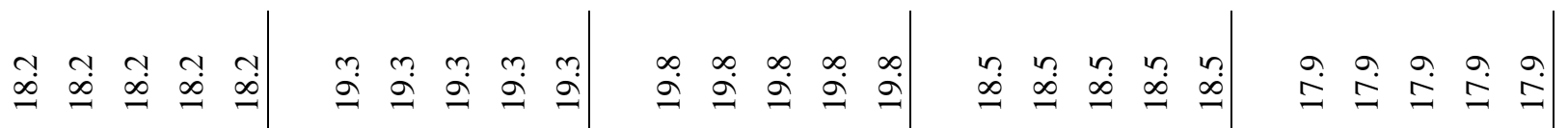

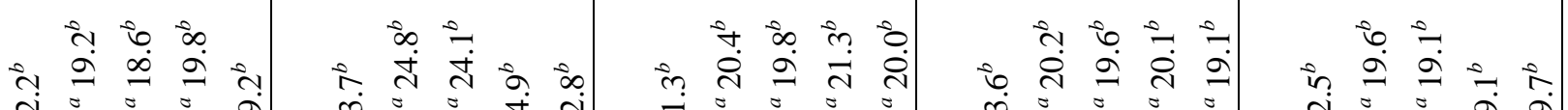

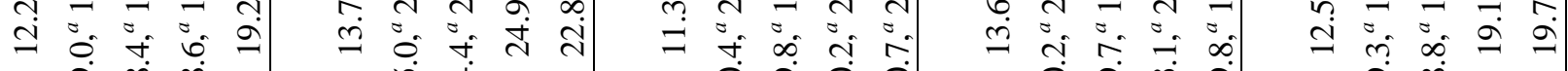
$\stackrel{\infty}{\infty} \underline{\underline{m}}$

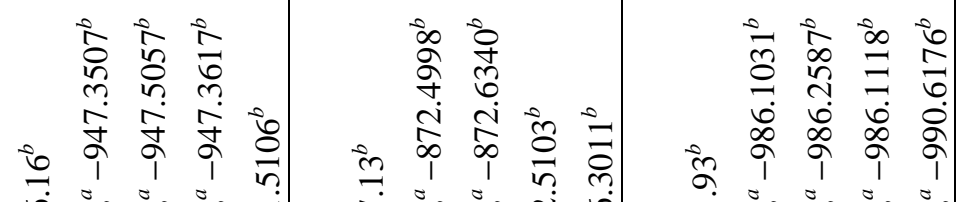

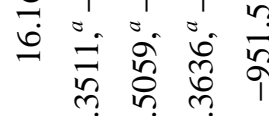
等等早

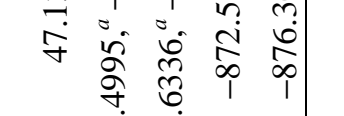
क्षे क्ष

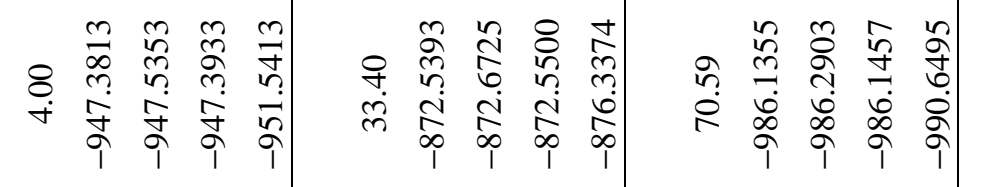

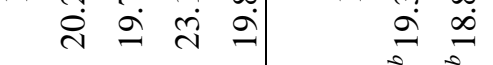

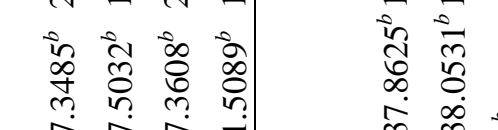

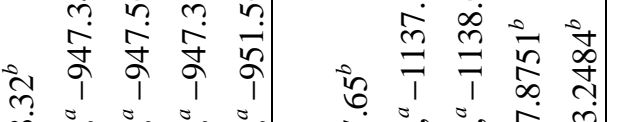

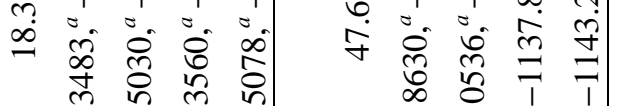

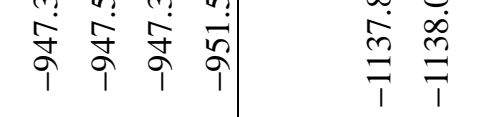

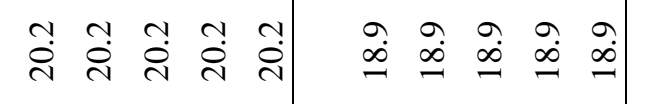

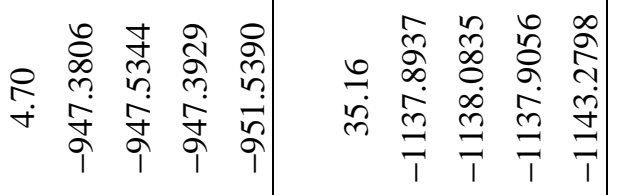

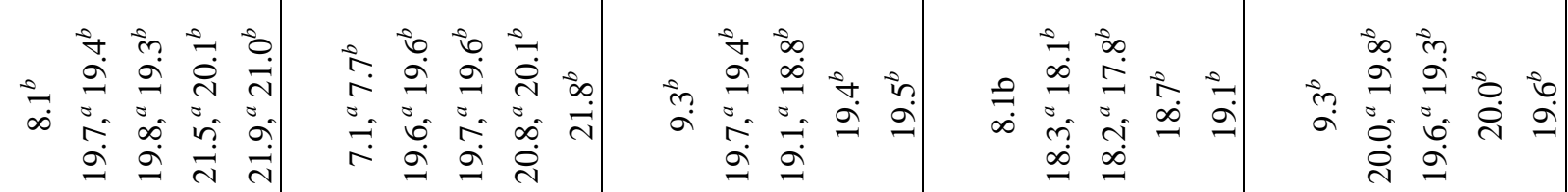

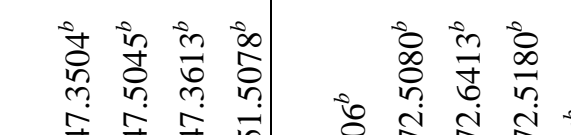

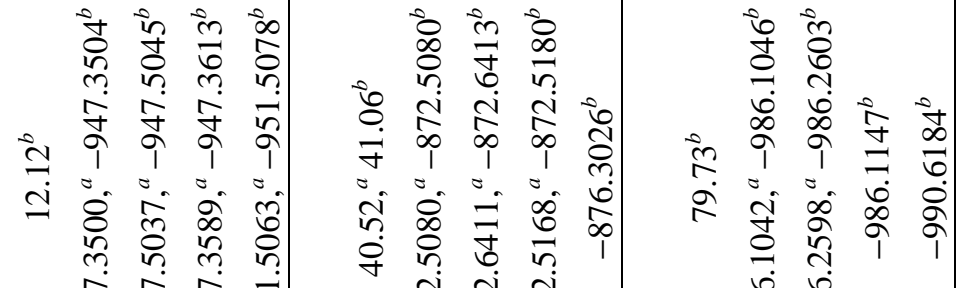

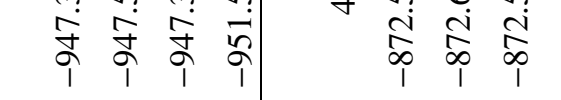

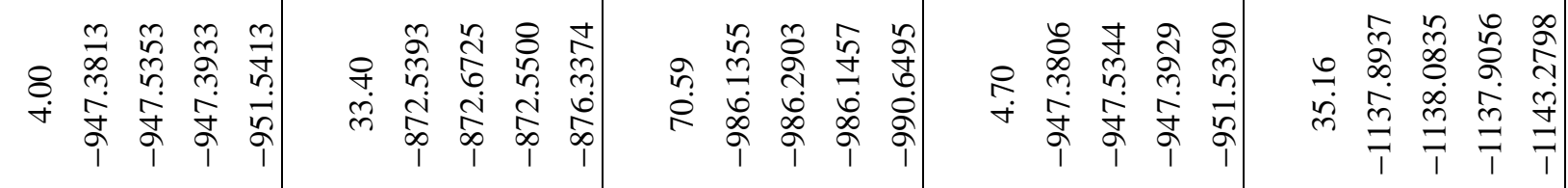

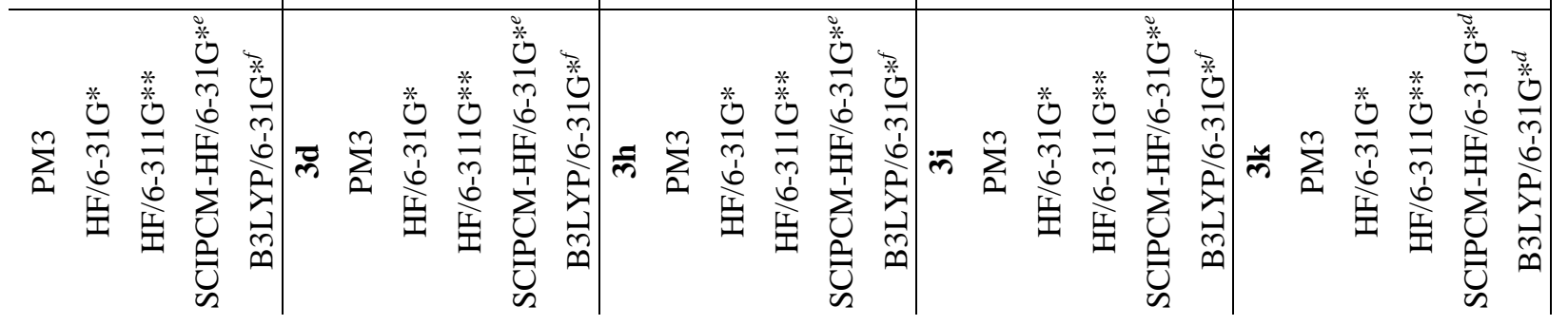


$\frac{n}{n}$

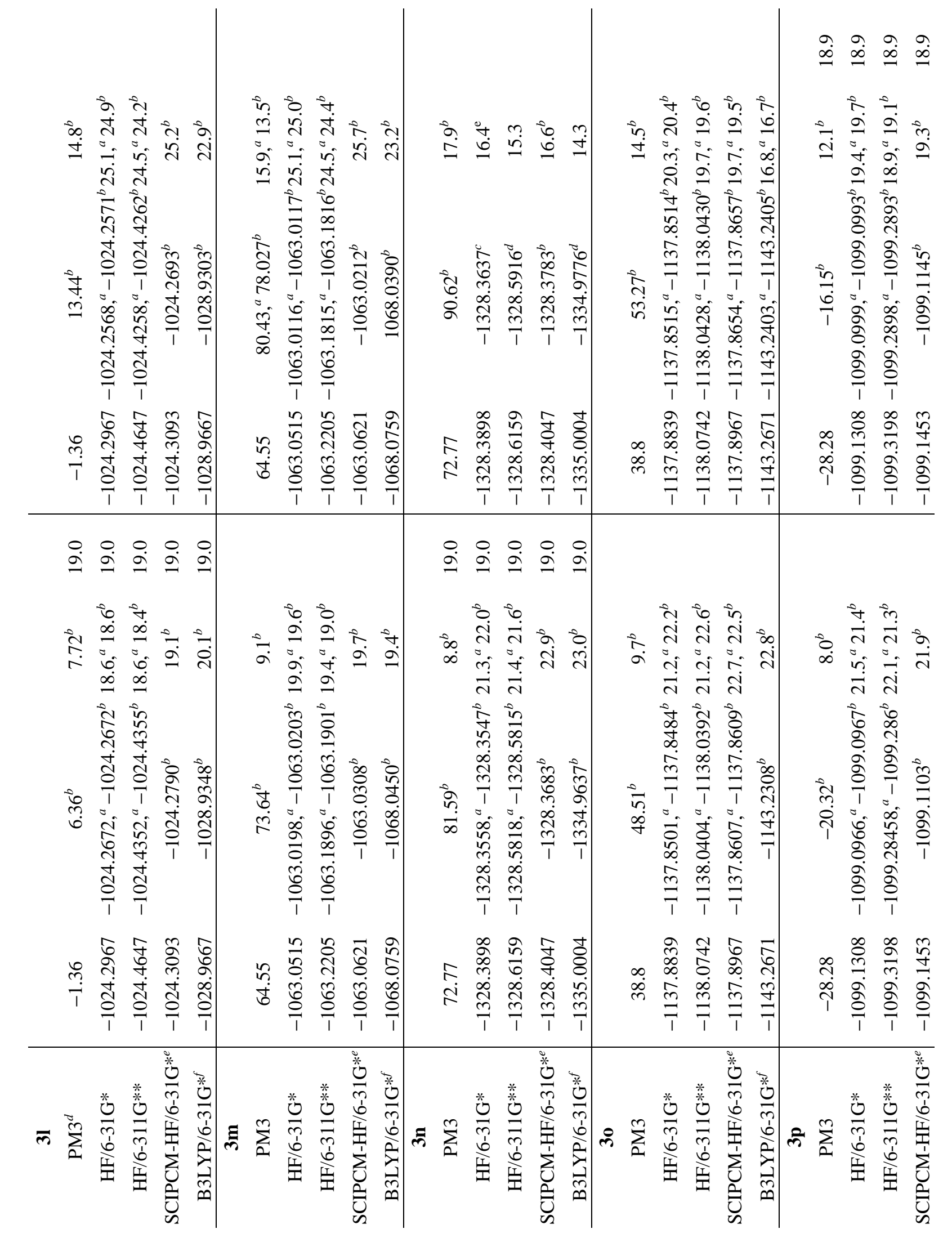


$\frac{6}{n}$

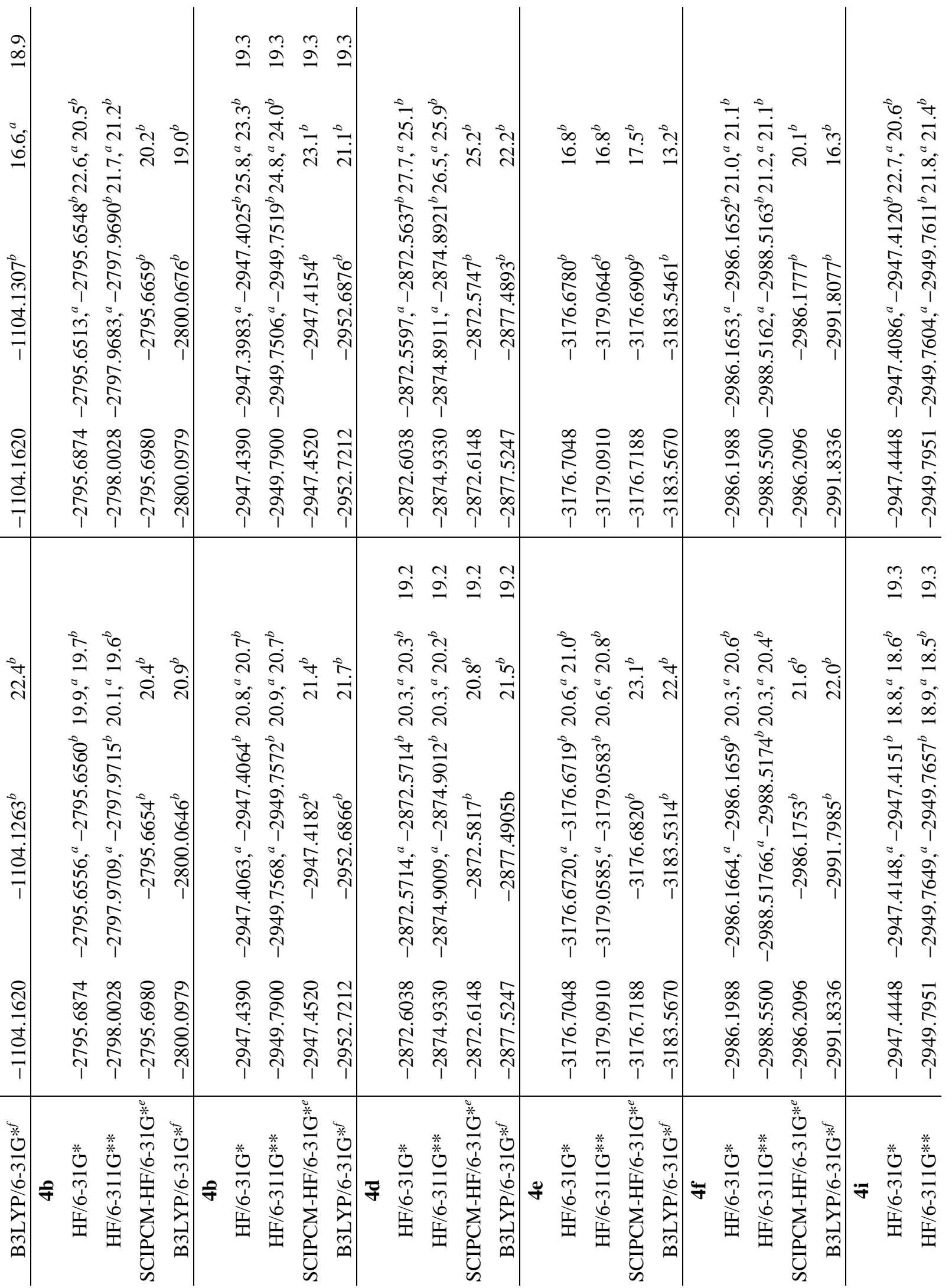


क

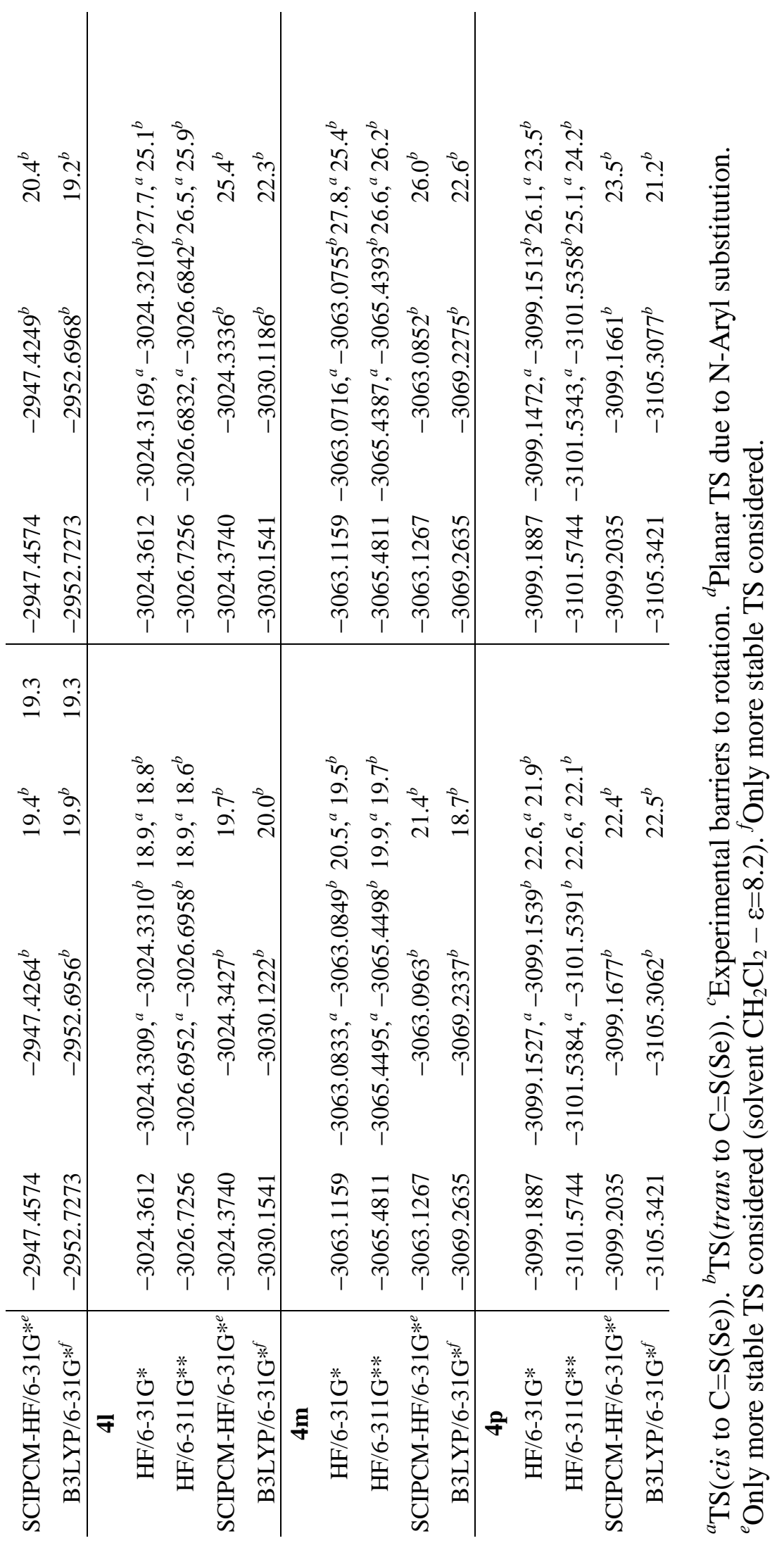


$\frac{\infty}{\sim}$

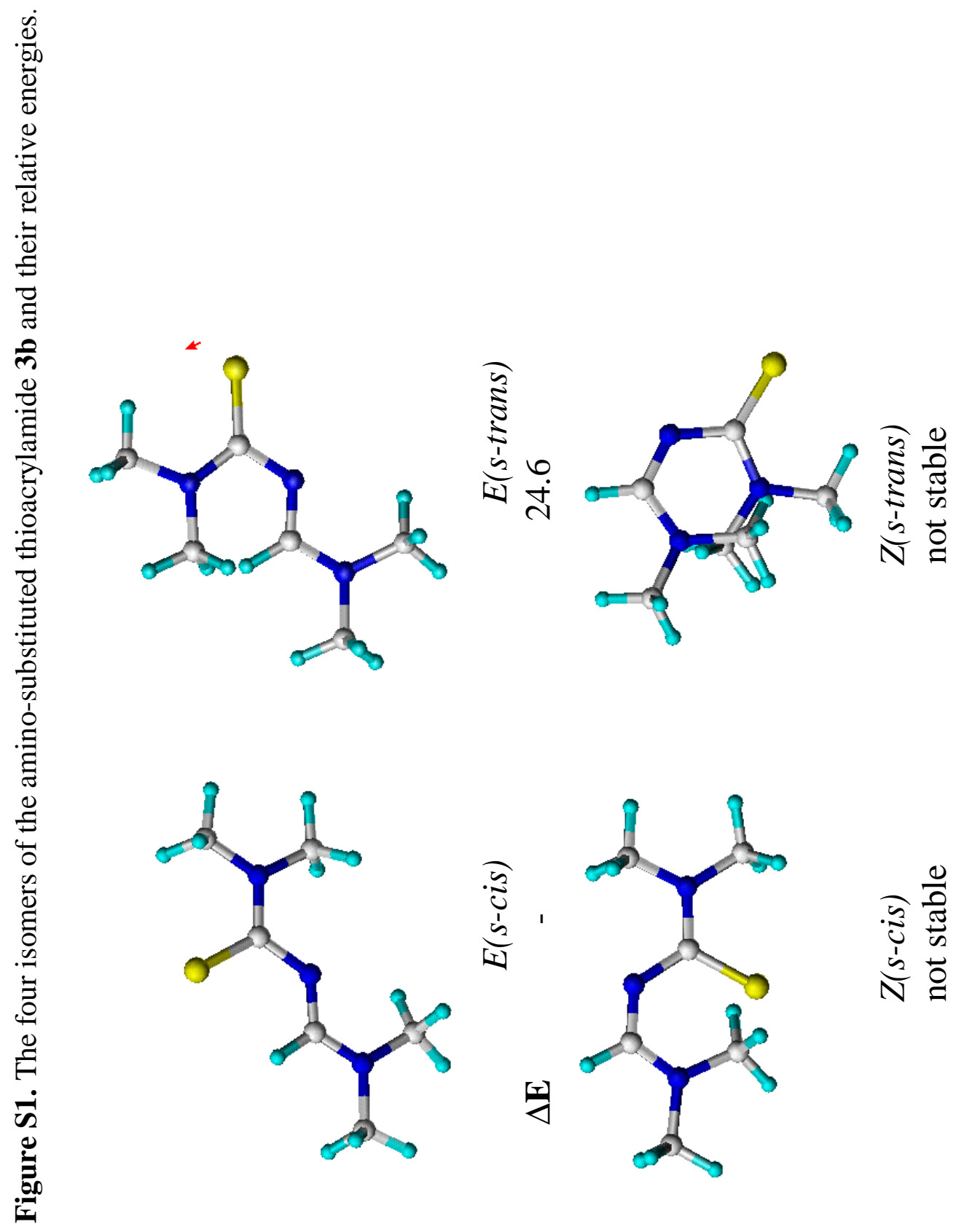


के

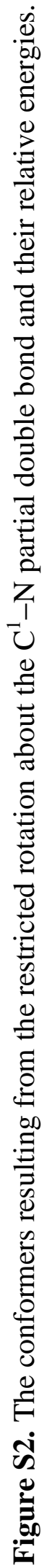
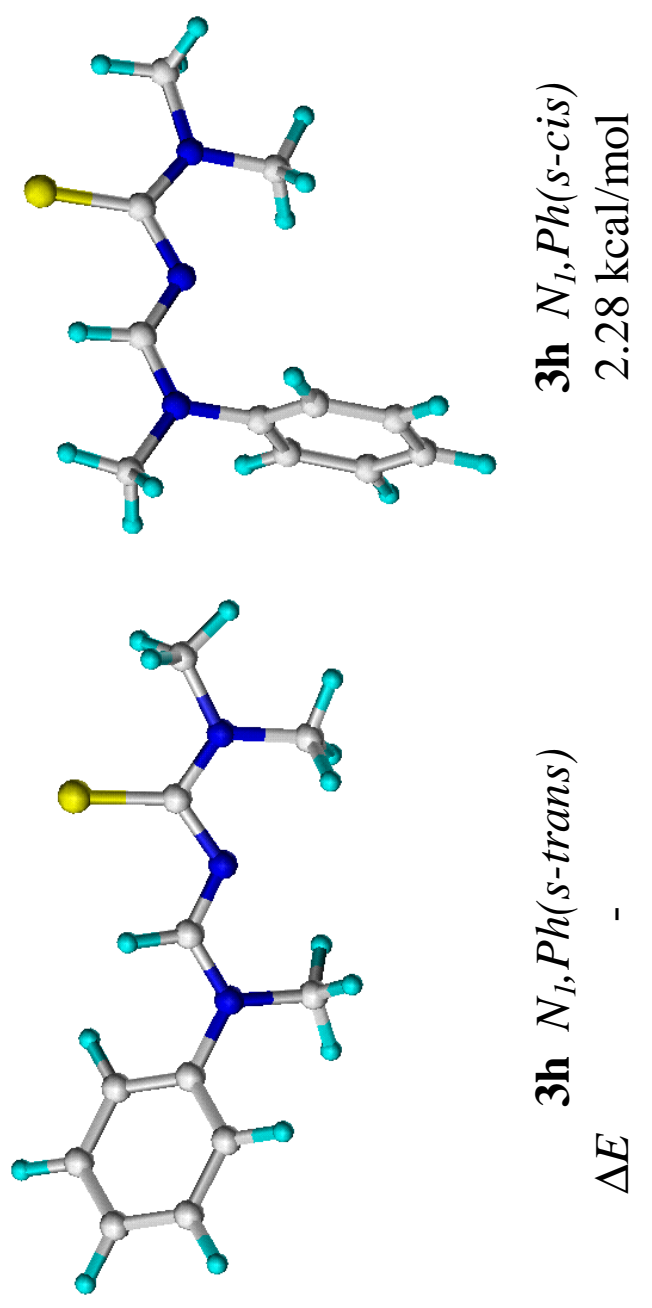
ฉิ

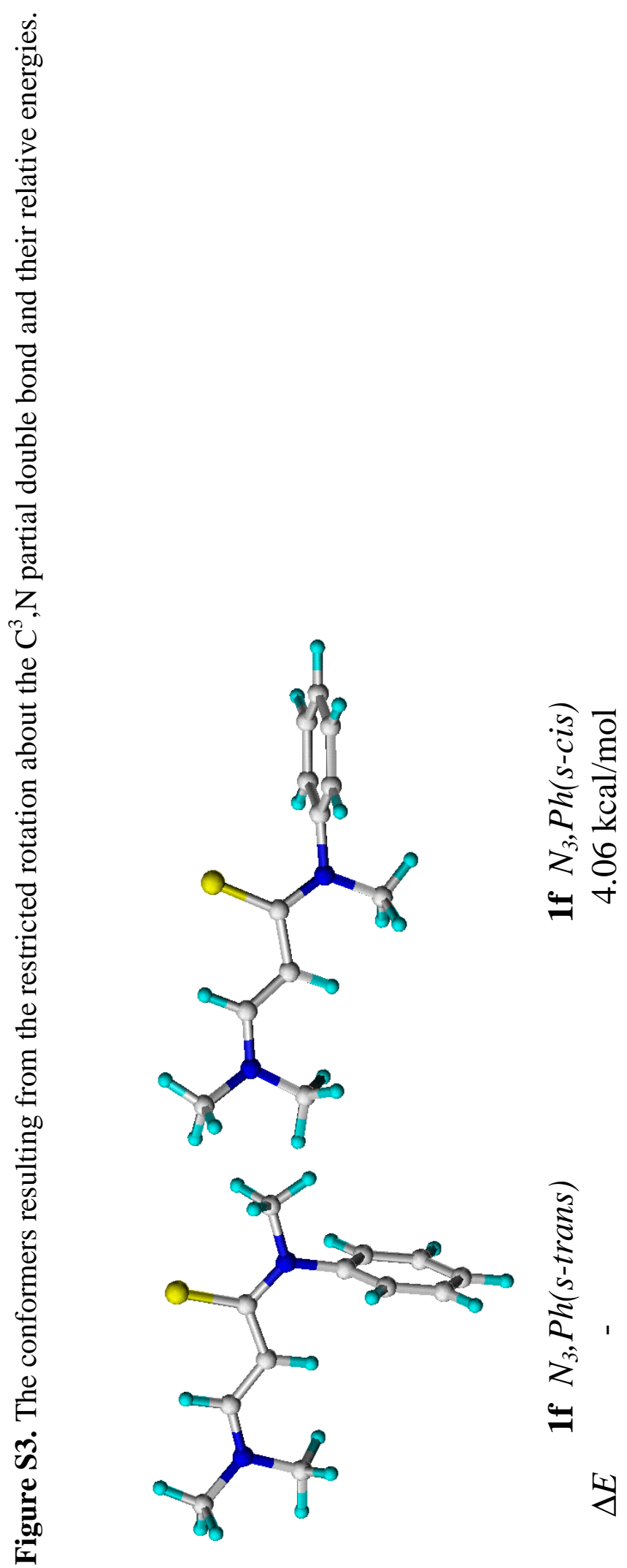


$\vec{\infty}$

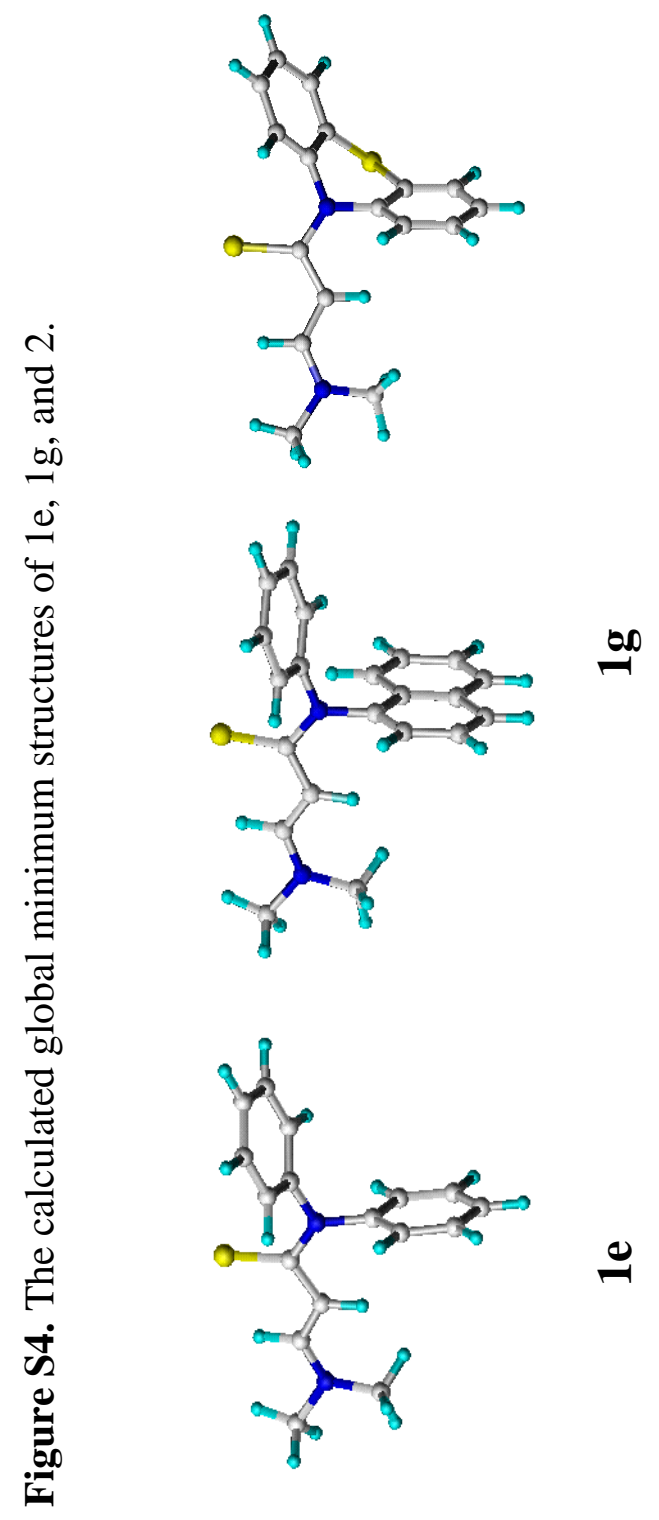


ฮี
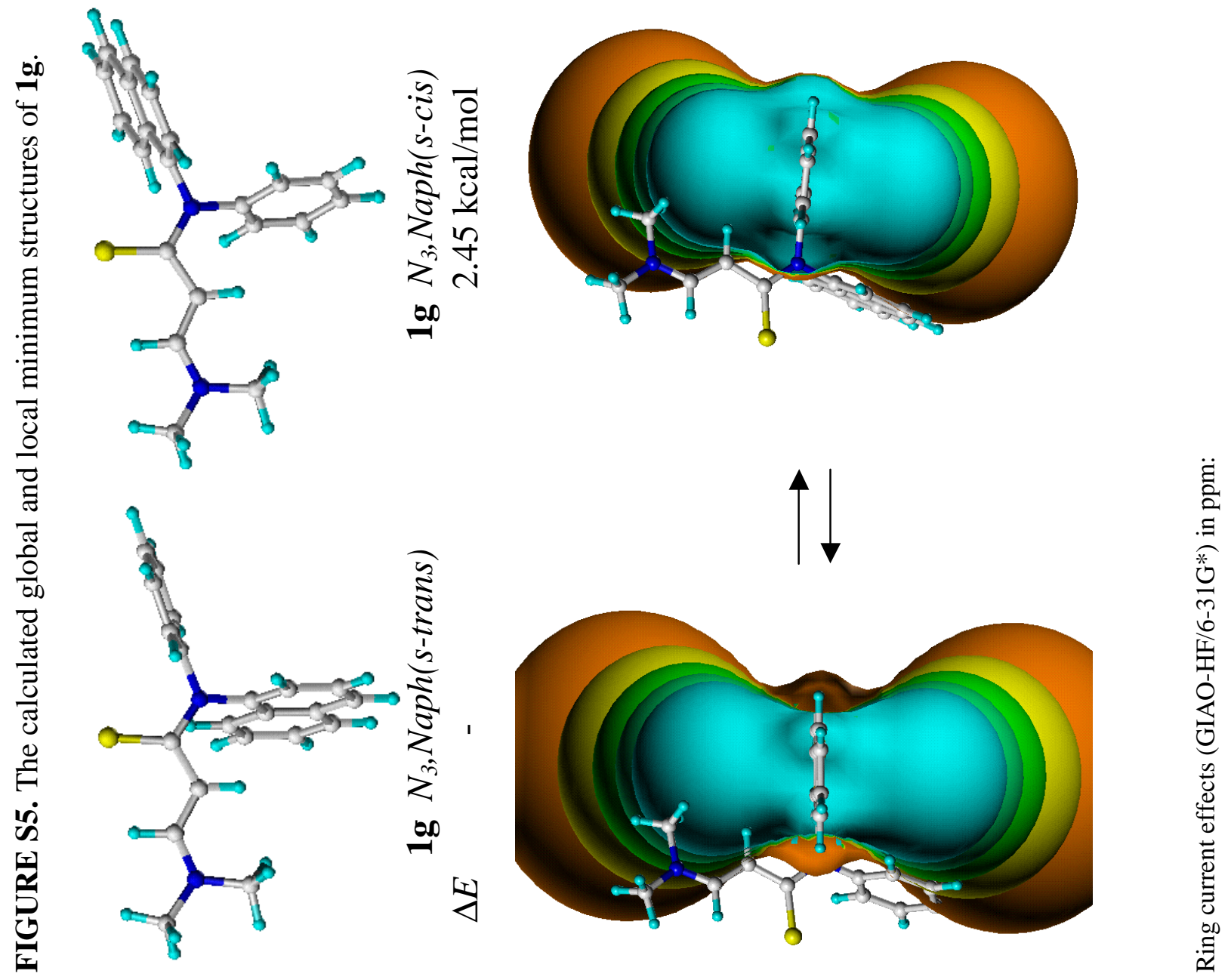
$\approx$

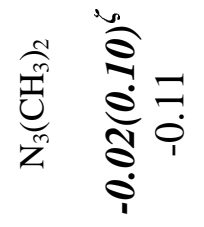

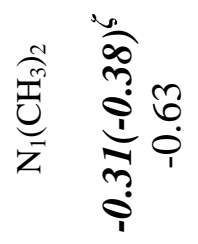

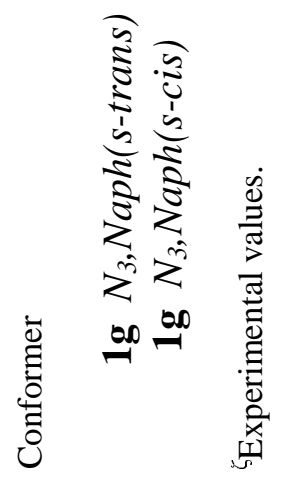




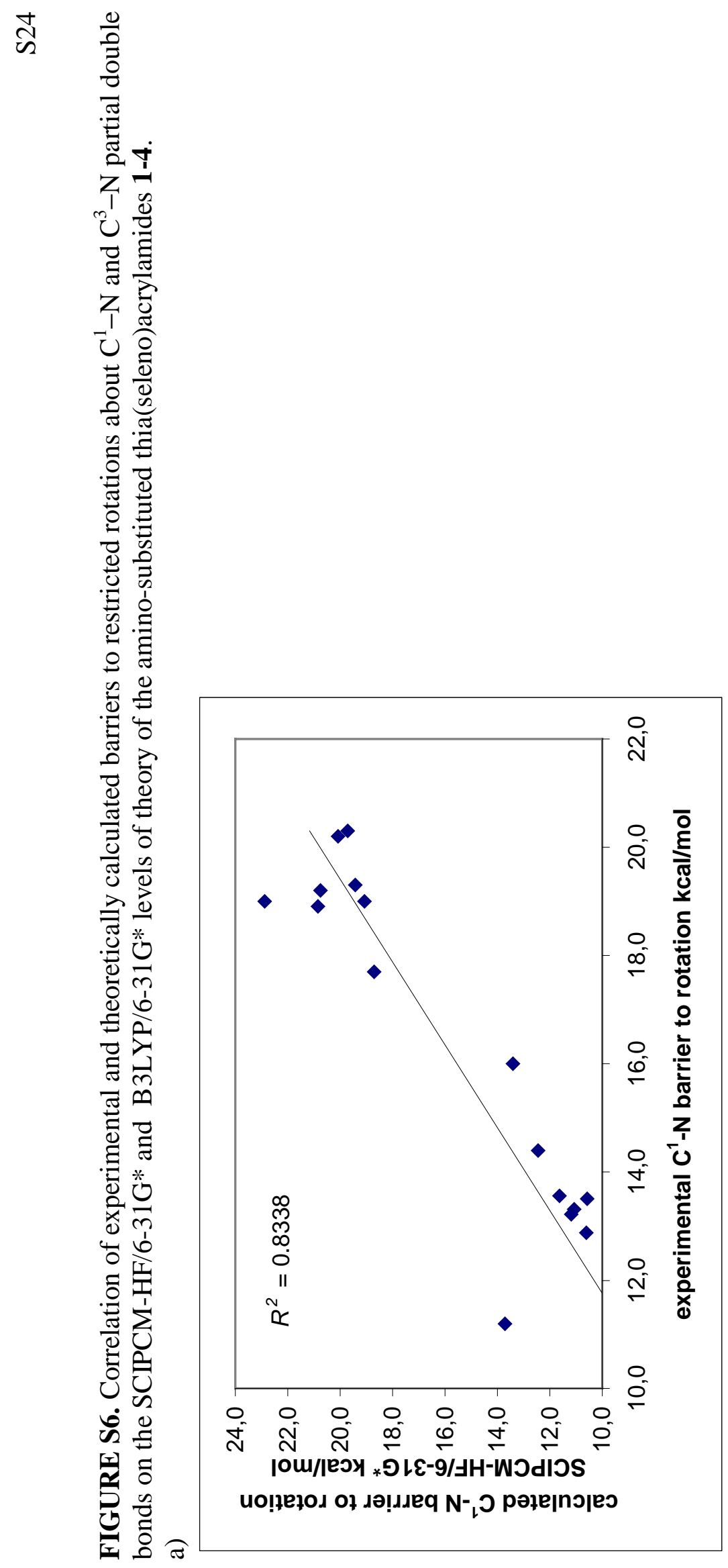




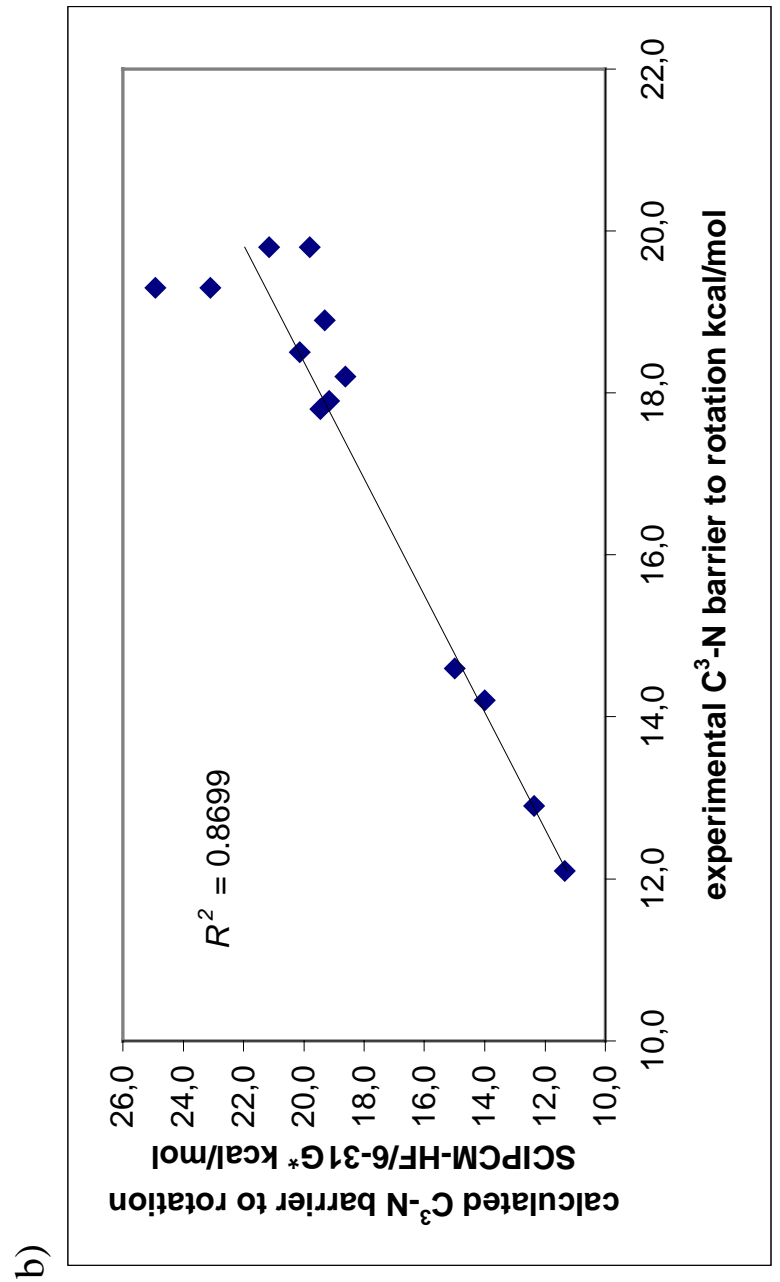



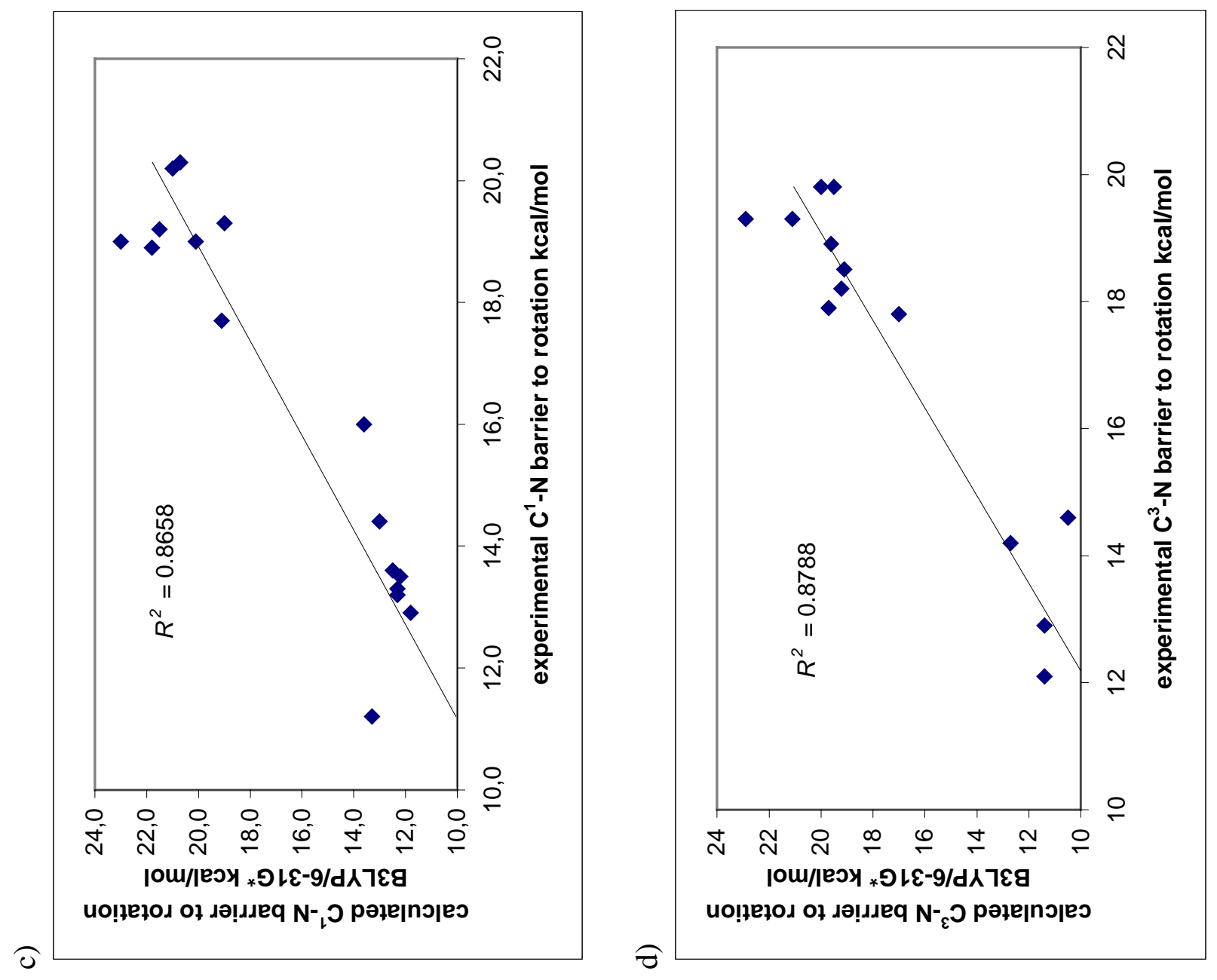


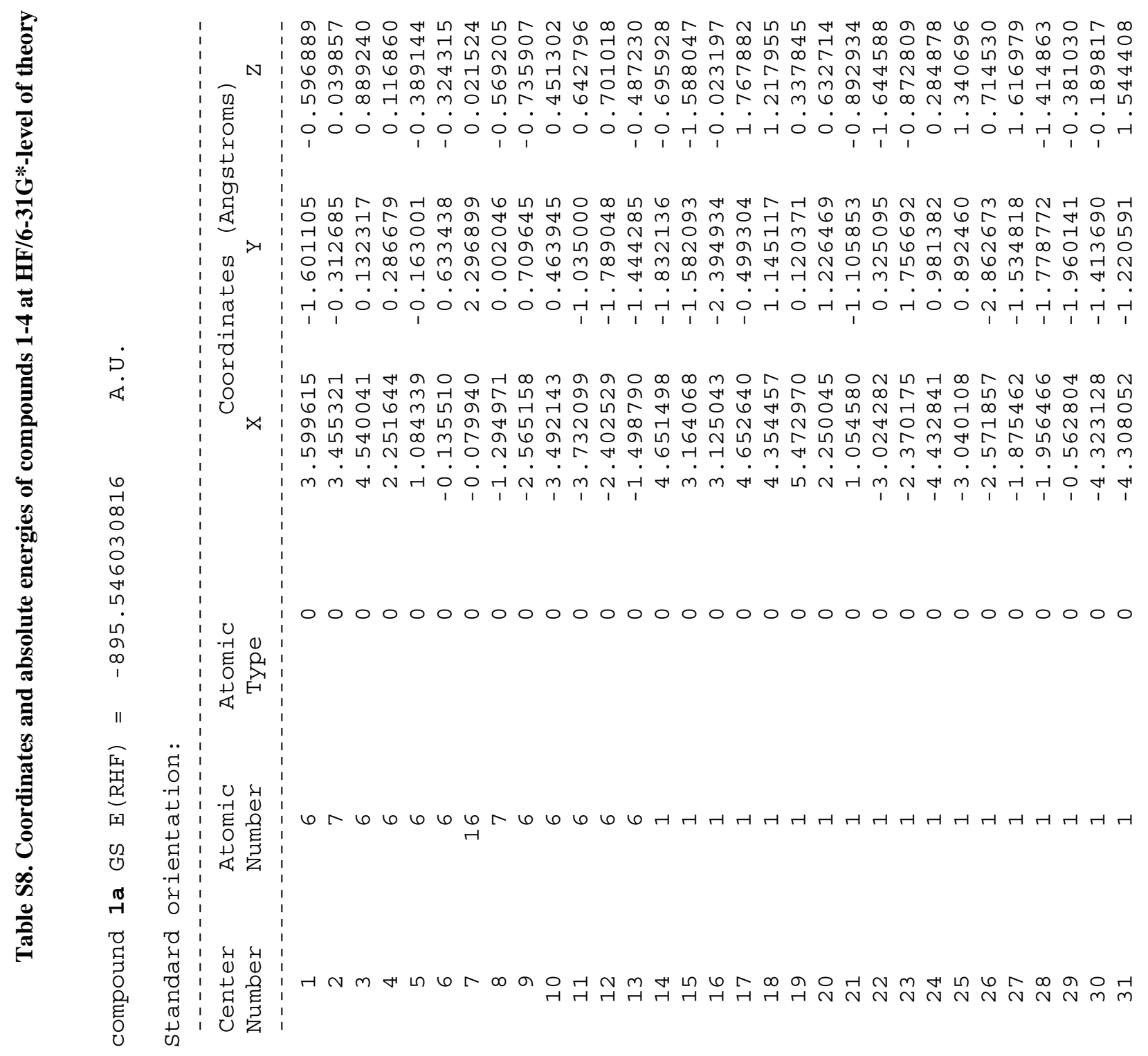




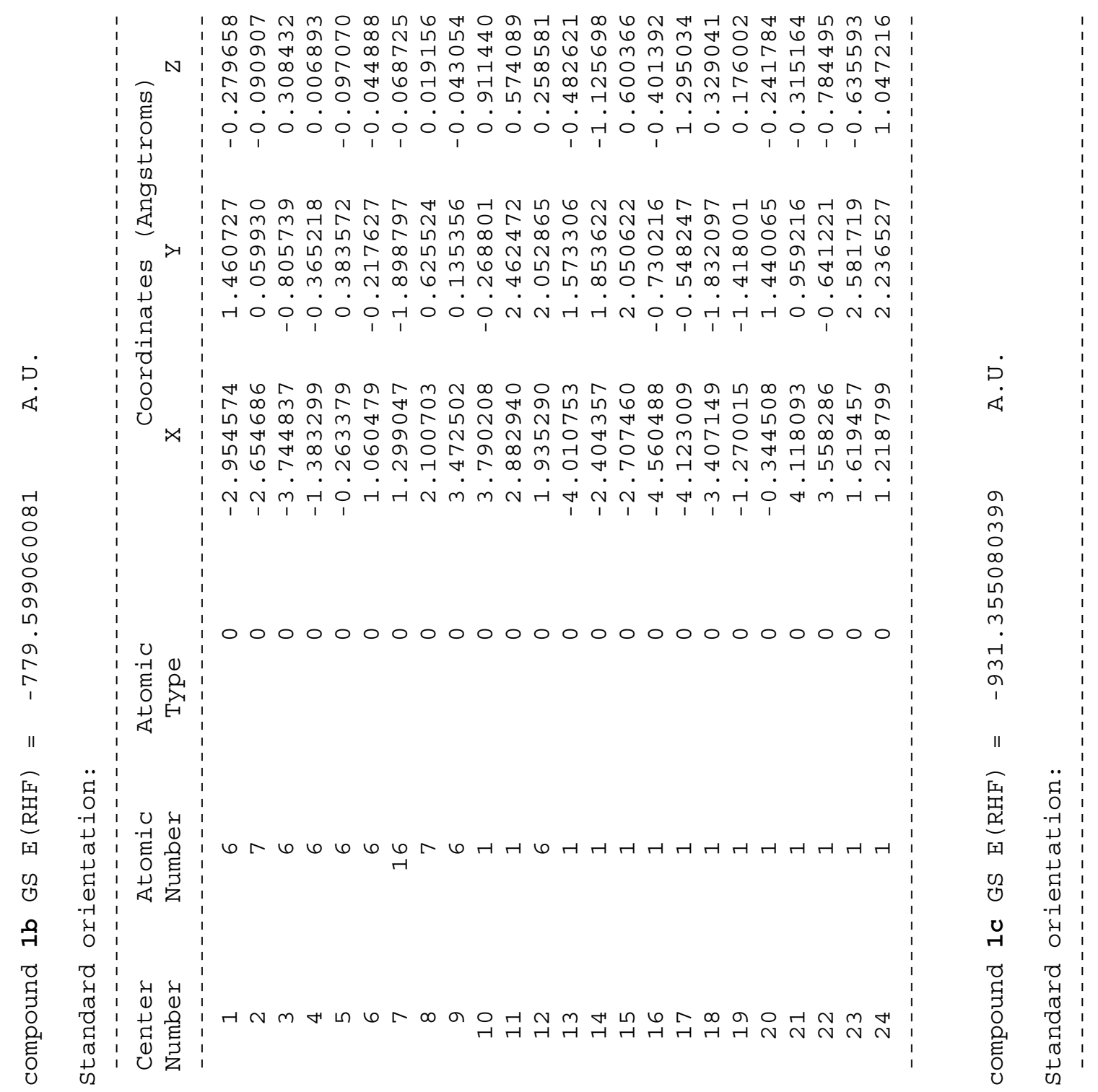


I

। $\infty N$ N $N$ H

$N$ i $\infty$ ब

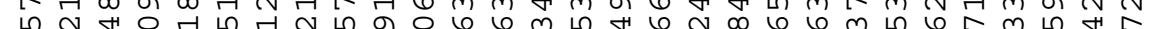
- 0 เ

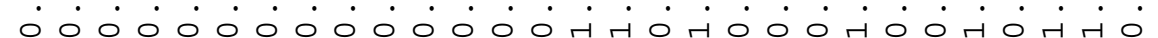

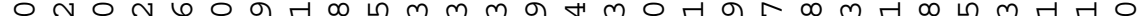

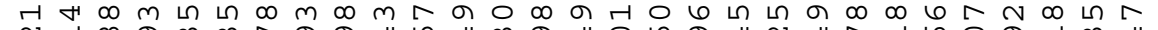

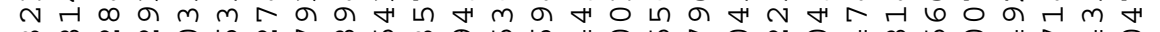

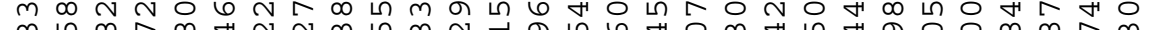

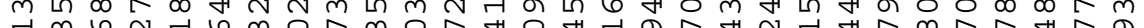

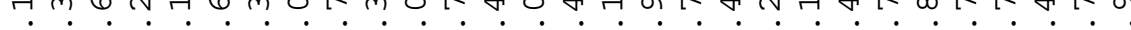

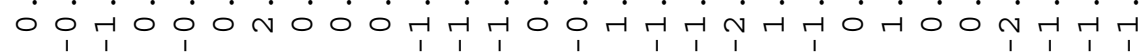

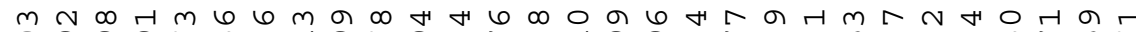
, व ब ब 6 ॠ I N

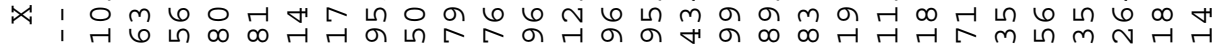
6 Hmm $\mathrm{m}$ - 00 H

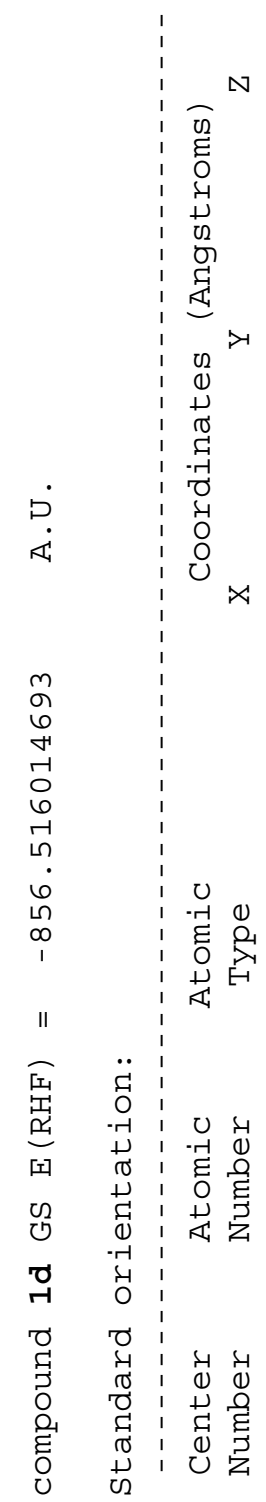



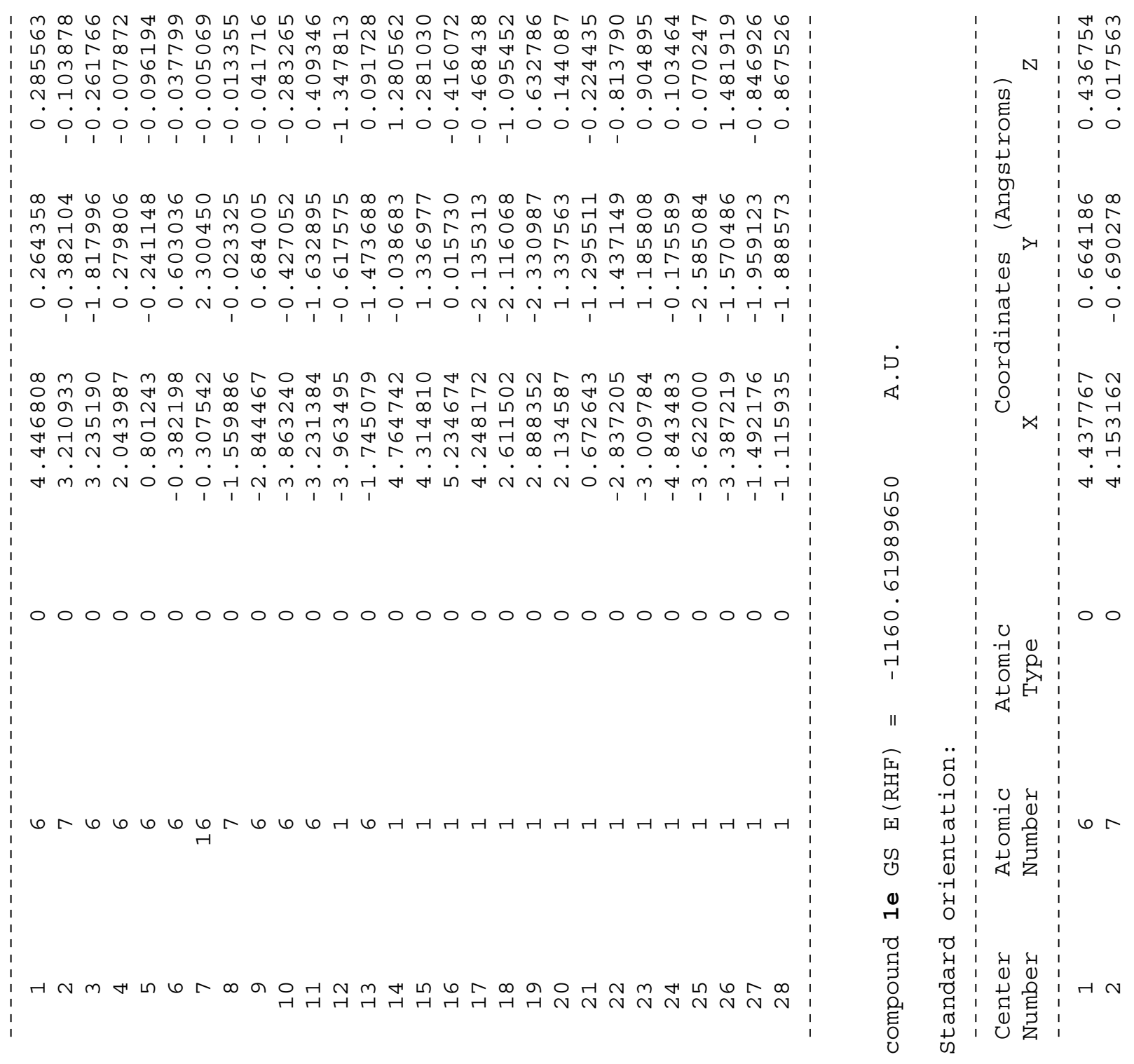
N

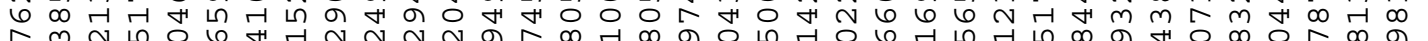

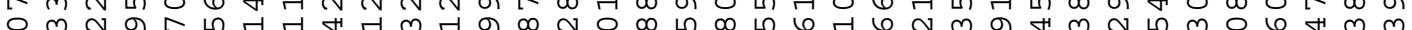

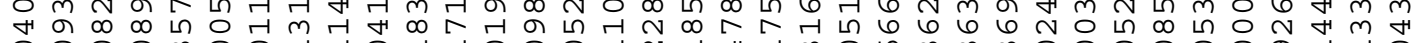

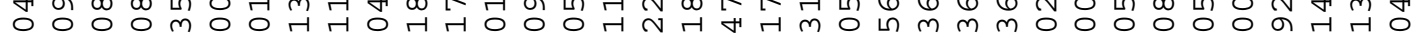

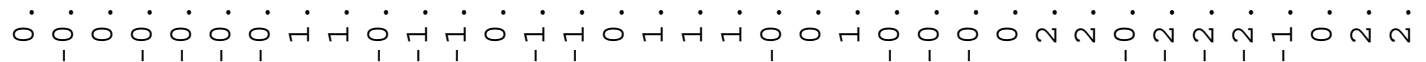

$\infty$ ம

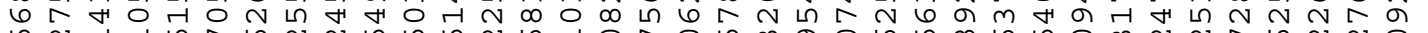

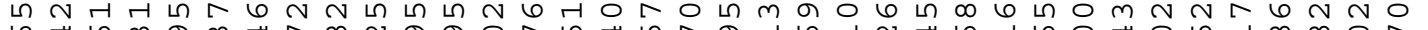
ம

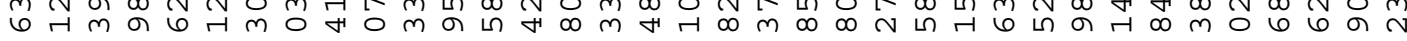

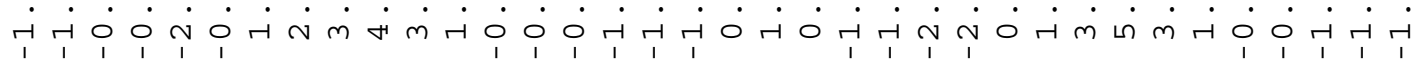

H H

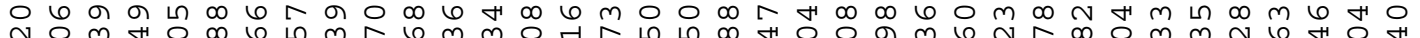

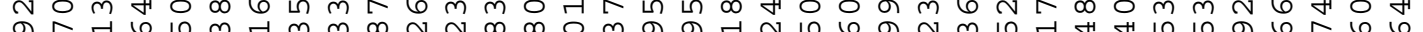

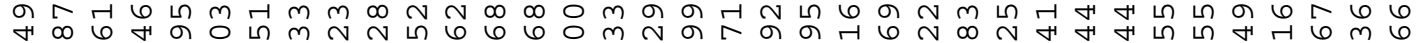
N $\infty$ H

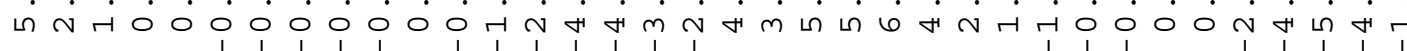
000000000000000000000000000000000000

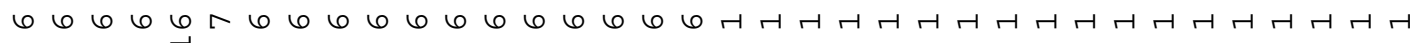
m 


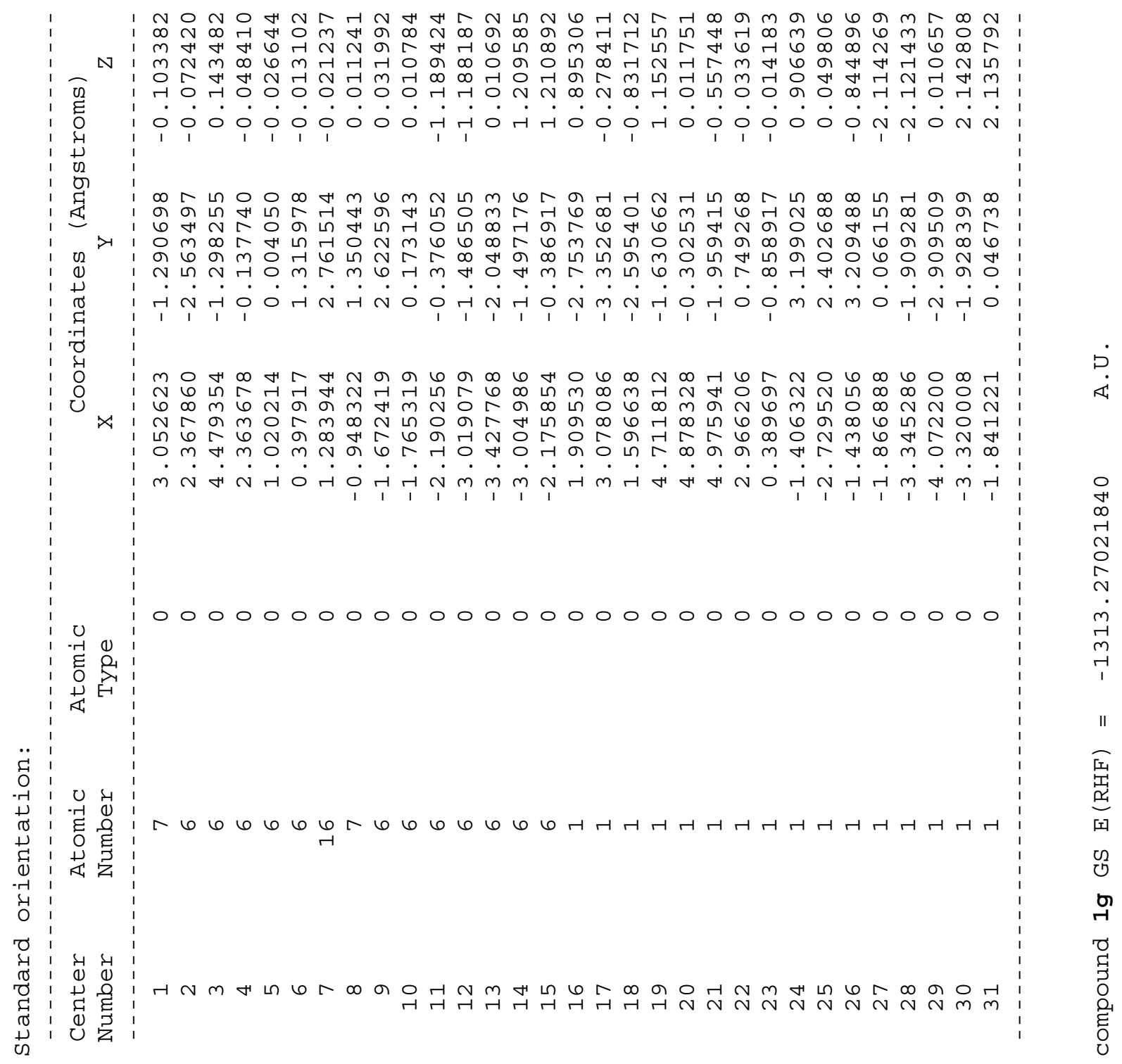




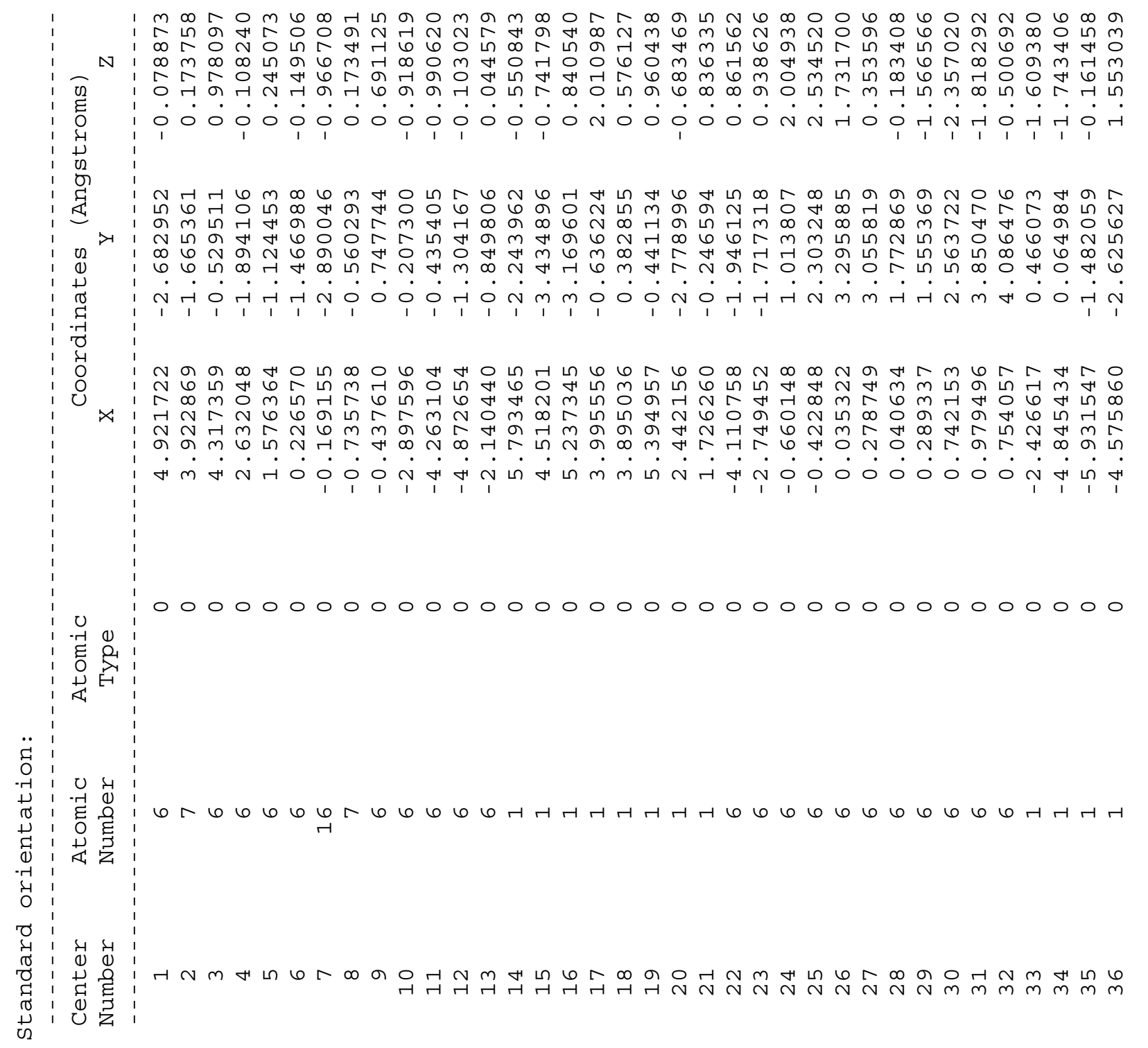


- $\infty \infty m$ เ 다 त्र $\circ m m \backsim N$ ก

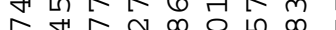
66 เ $\dot{\sim} \dot{\sim} \dot{m} \dot{\sim} \dot{m} \dot{0}$

6 अ 6 ก $\mathrm{m}$ - 0 अ $m$ ब

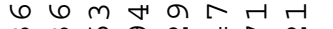
$m m\llcorner$ व $N$ H $N$ i $N m \infty \Gamma \infty \infty m b$

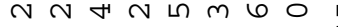
$\dot{\sim} \dot{\circ} \dot{\sim} \dot{\circ} \dot{O} \dot{H} \dot{0}$

$6 \mathrm{~T}$. 0 त 6 क 0 \% ก म

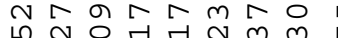

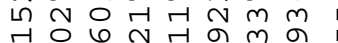

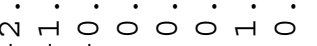

00000000

म 4 म

$\hat{m} \infty m$ の

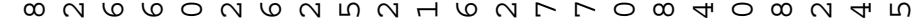

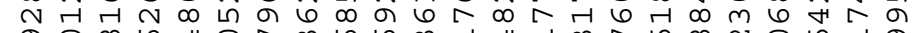

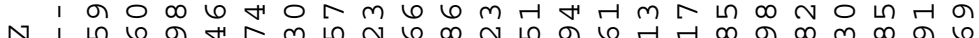

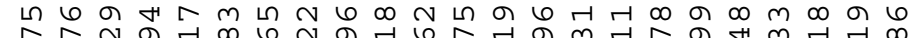
N A 00 H 0 H N

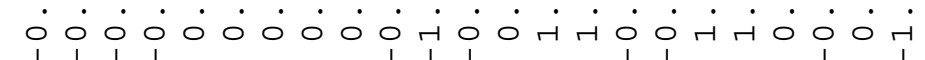

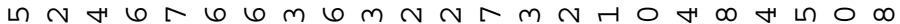

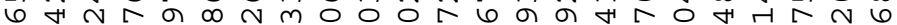

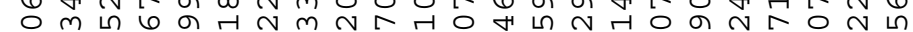

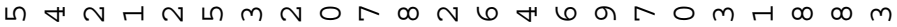

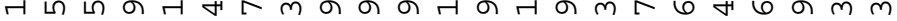
两

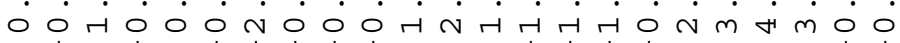

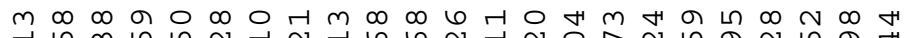

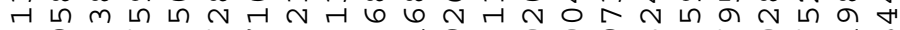
स

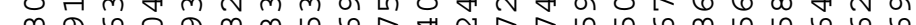

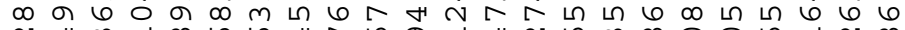

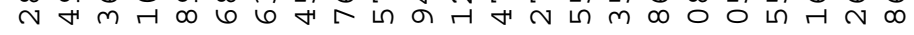

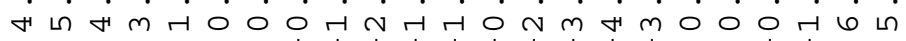




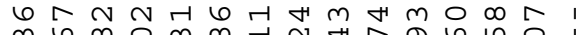

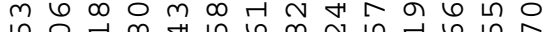

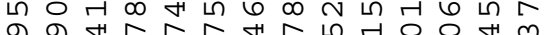
ब बे $m$ म $m 000$ ब

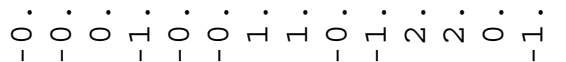

$\infty$ எ

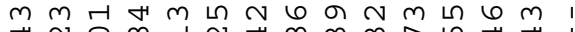

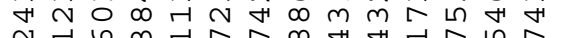
N

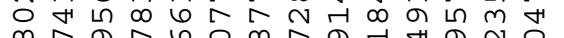
$\infty$ の

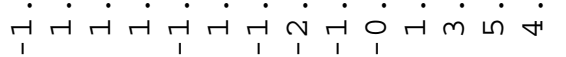

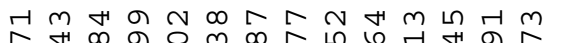

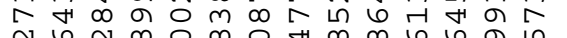

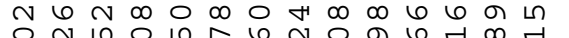

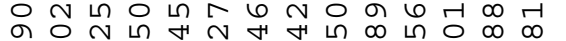
ฟ

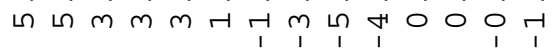

00000000000000

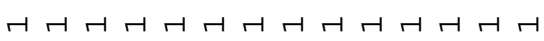

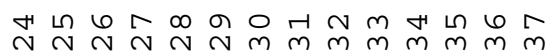

I 6 の N $10 N$ in $0 m$ H $m$ H 6

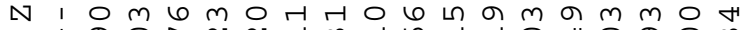
ब $0 N N$ H $m$ म

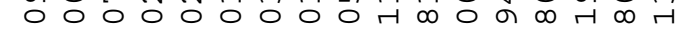

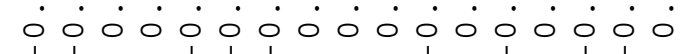

$m m 066$ m $6 \mathrm{~m}$ の

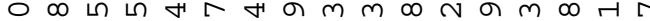

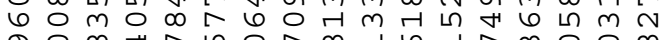

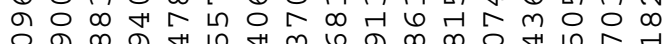
$\circ \sigma \infty \sigma$ ॠ

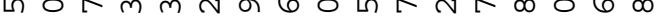

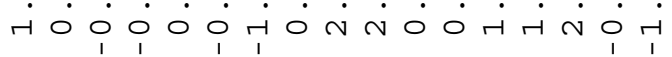

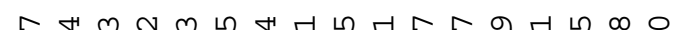

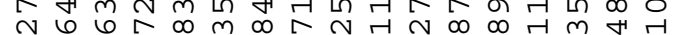

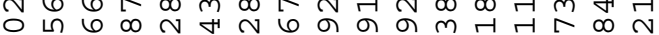

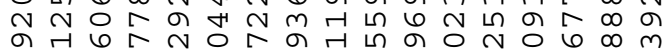
の $\dot{N} \dot{m} \overrightarrow{0} \dot{H} \vec{H} \dot{H} \dot{m} \dot{m} \dot{m} \vec{r} \dot{m}$

II

焉

द्व U H 鼻 0

00000000000000000 H 


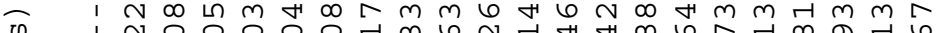
, N O O O O 0 H m 6 N

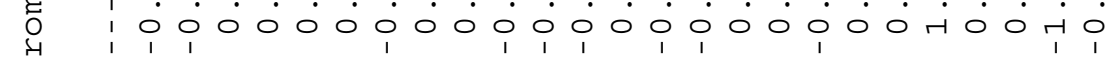
+ U

4

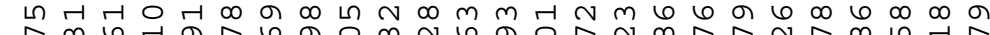
ก $m 6$ त

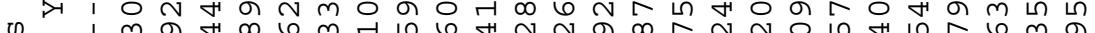
(1) $m$ n

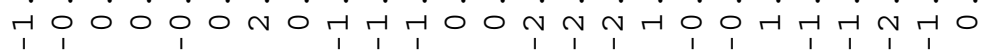

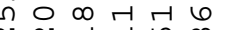
$N \propto \mu r$ L $\infty$ मतन

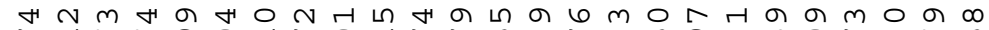
त

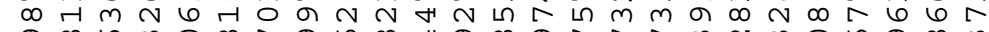
$m$ เा बढ ब मत r $N$ L

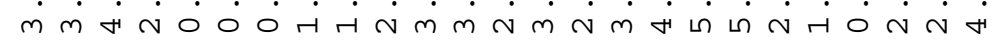

H $斤$ -

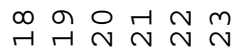

II

$\overparen{5}$

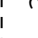

0000000000000000000000000

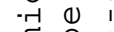
Oे

$\ddot{1}$

Ci

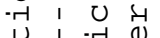

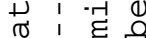

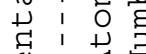

ช

0

.

б

空

6 


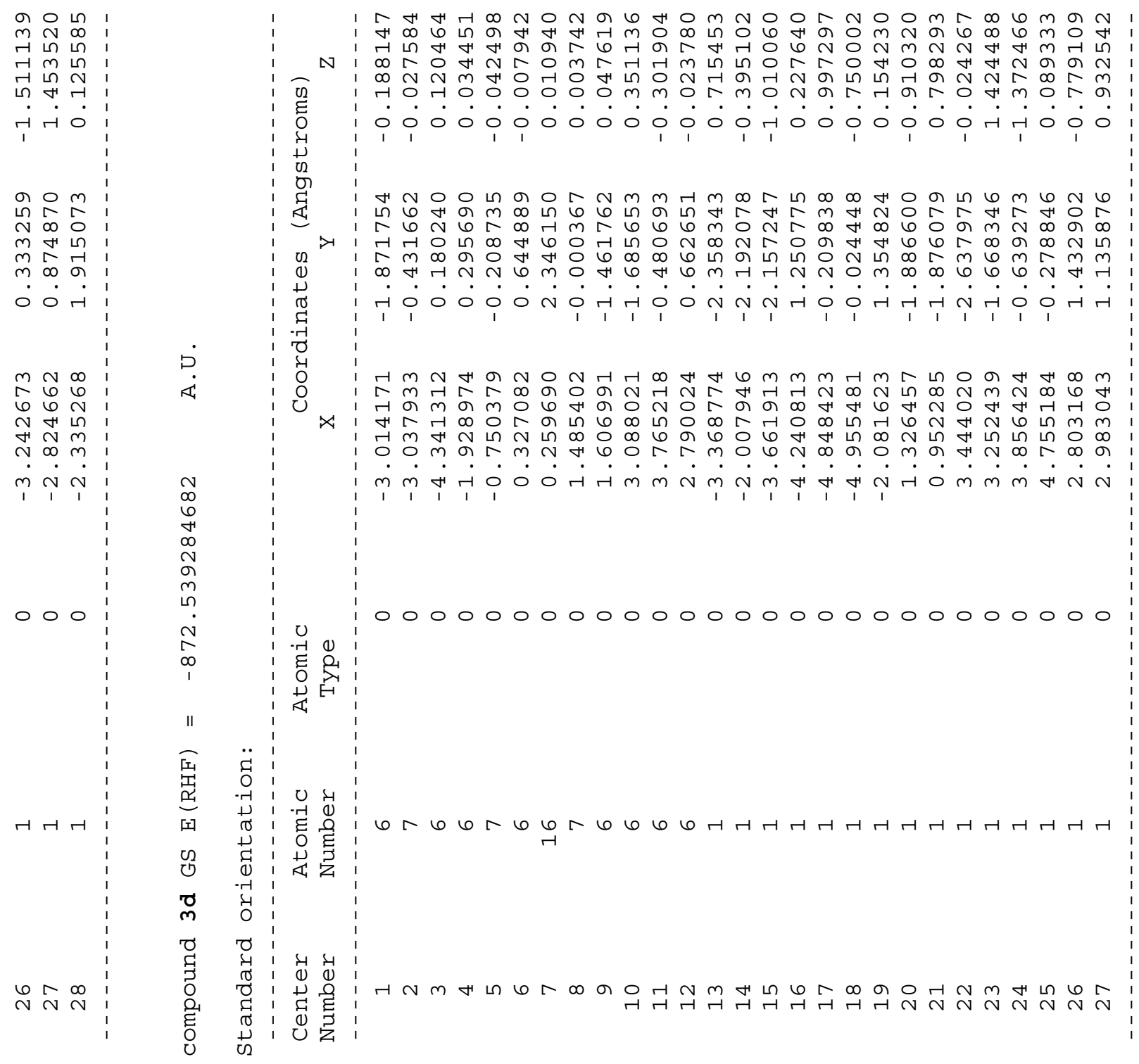




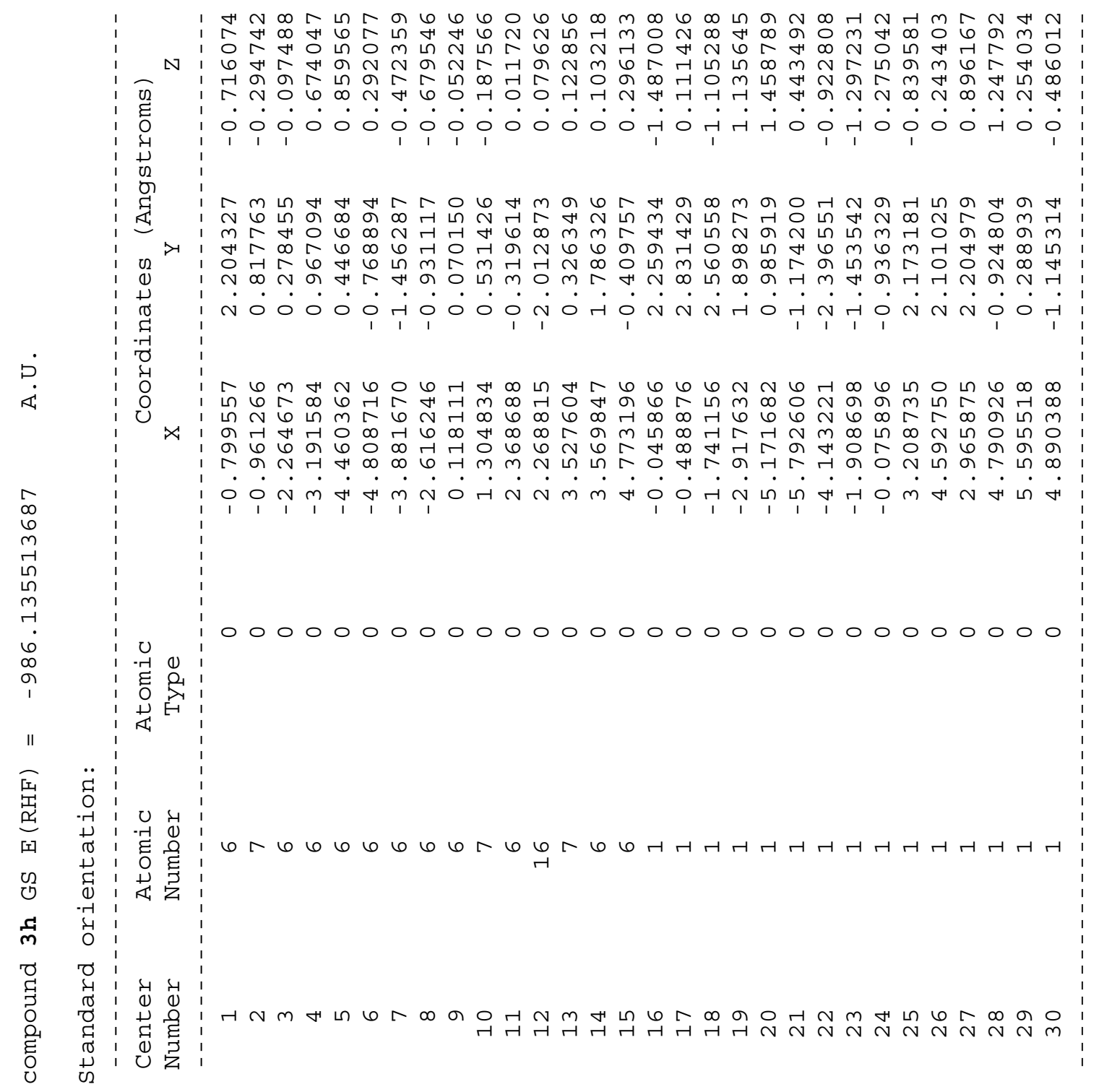




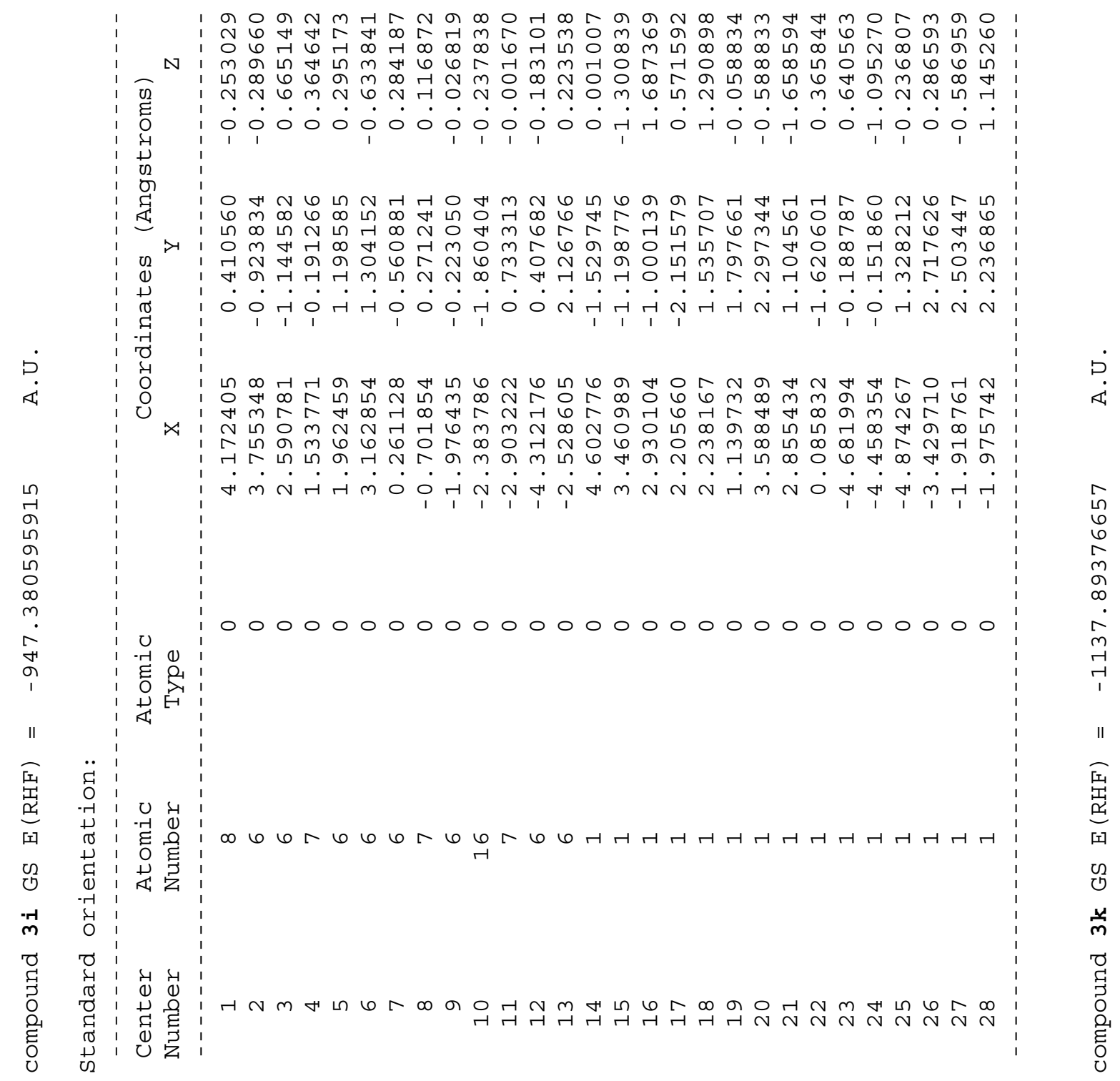




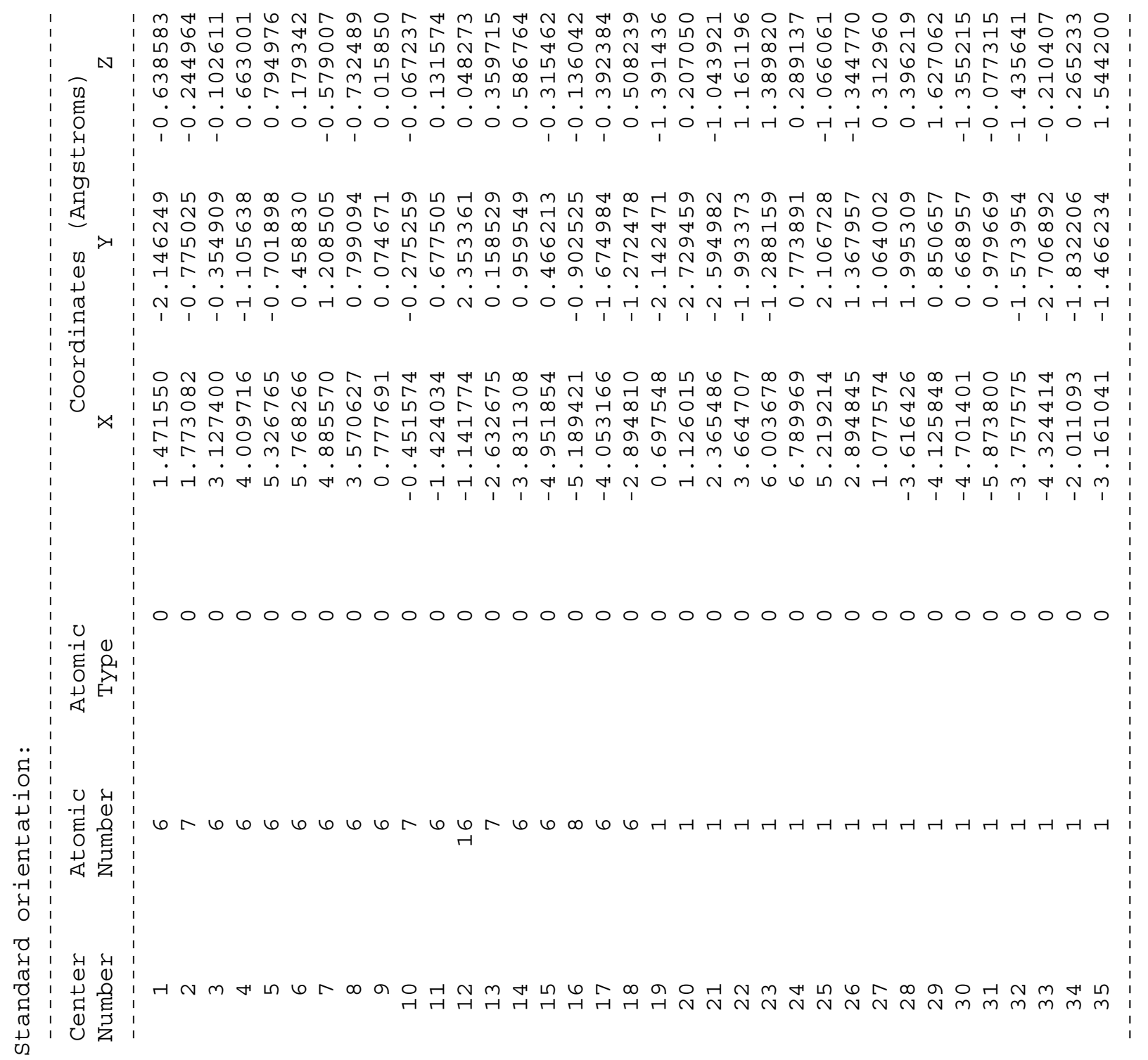




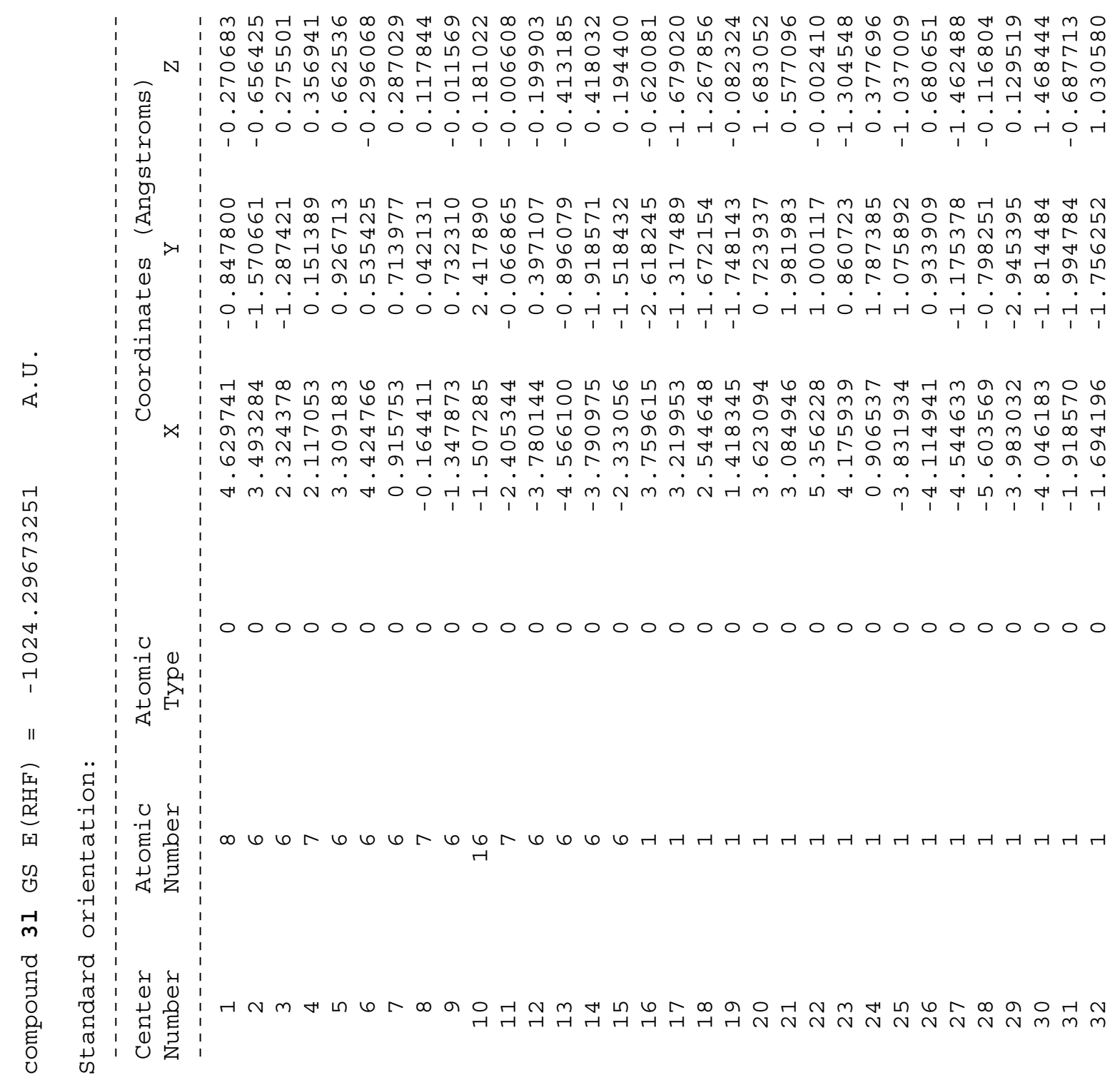




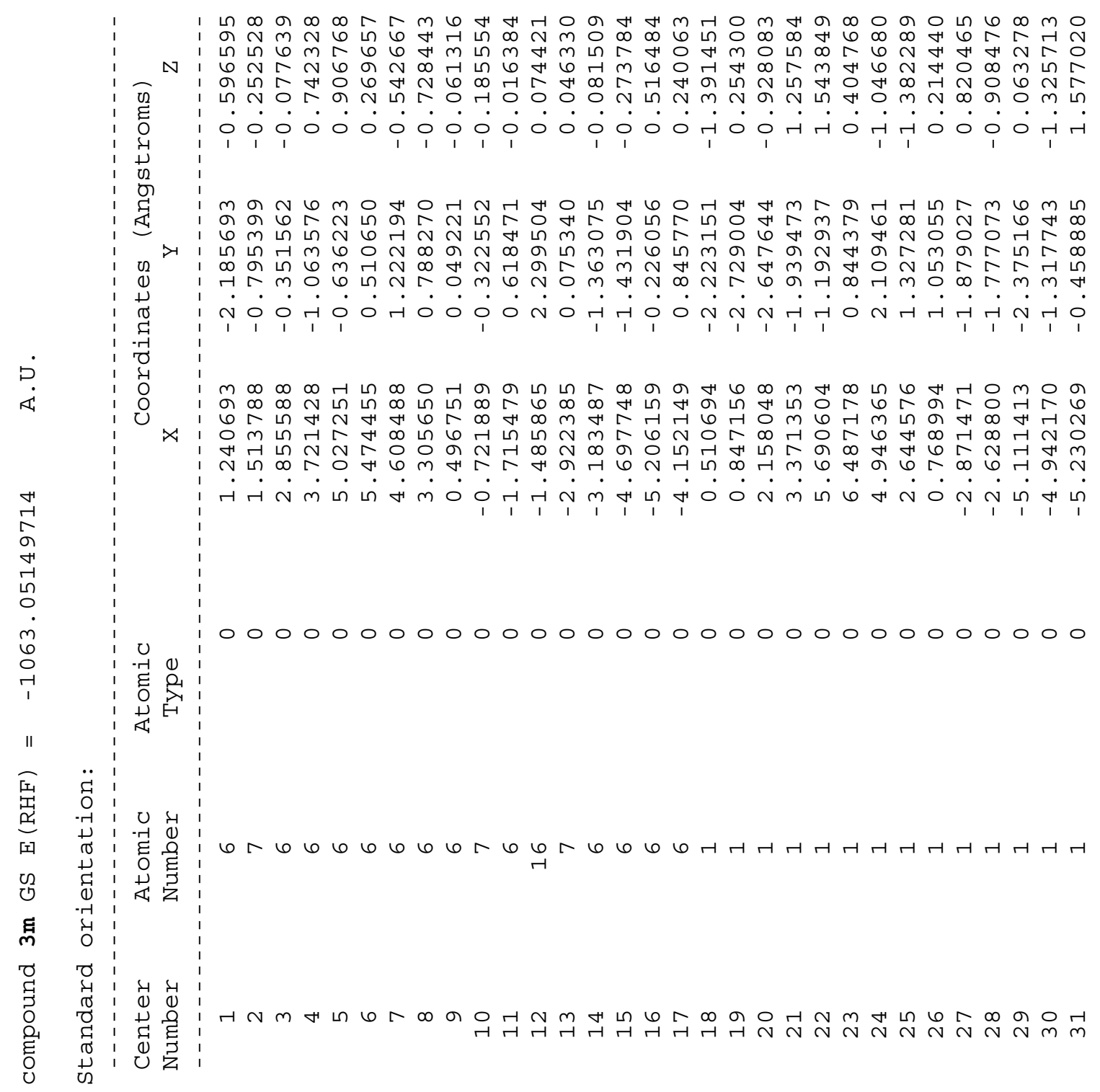




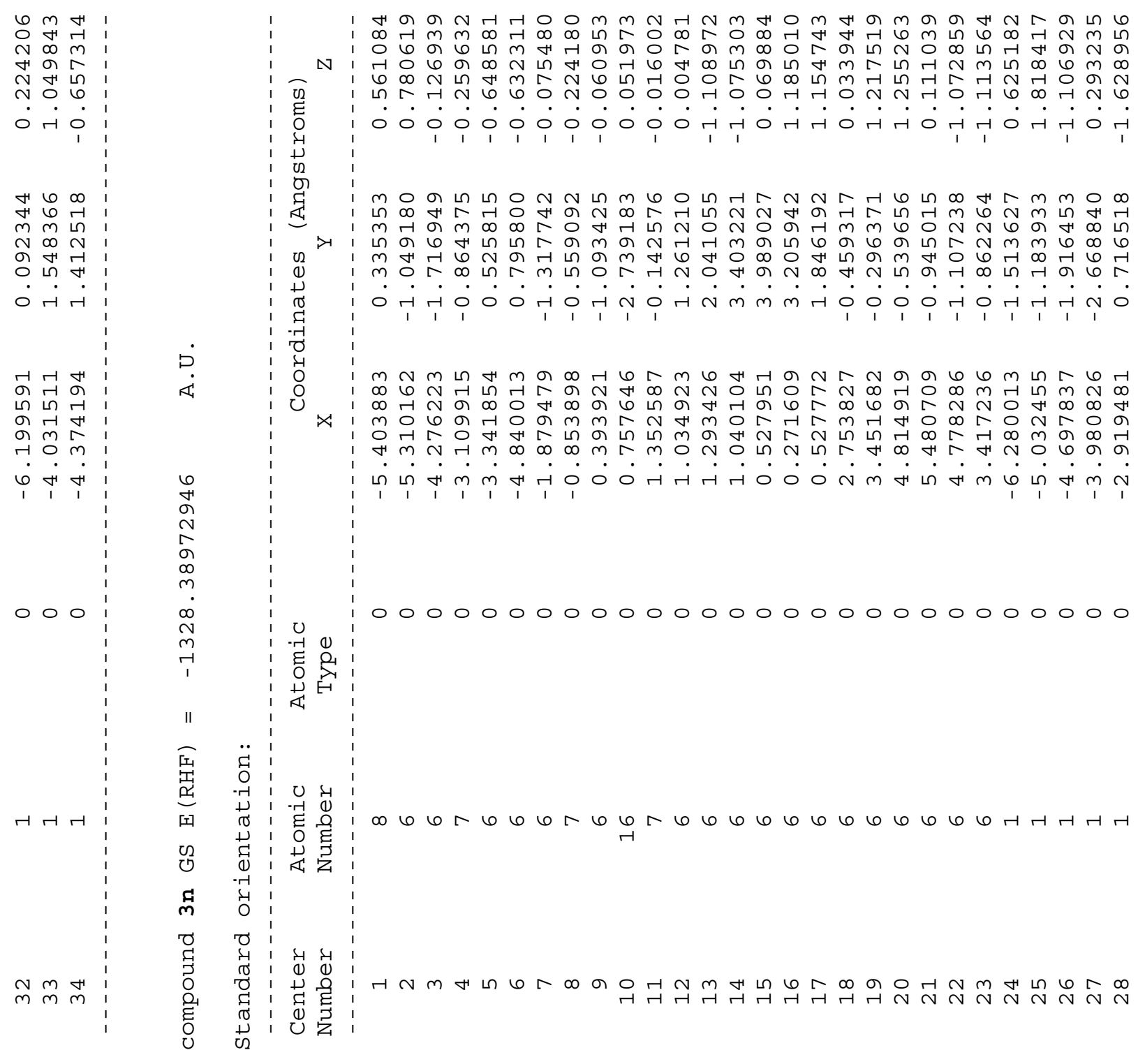


○ 06 เ

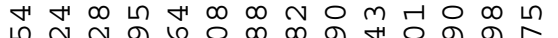

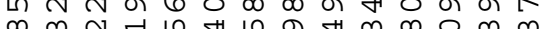

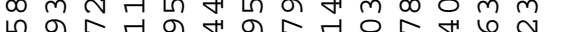
0 म 6 ब न 000 त

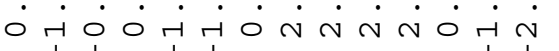

$0 \infty$ a N N H MONOMN $\infty$ L 6 H

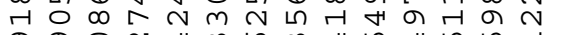

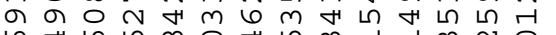
6 अ 6 เ $\infty 0$ अ न $m \infty m$ 几 $006 \mathrm{~N}$ म

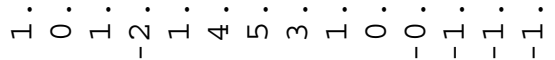

$N O \infty \operatorname{dn}$ N $\infty$ G $m \circ m$ L $\infty \infty m+6$ म $\infty$

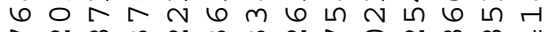

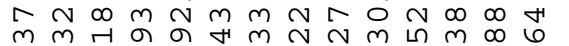

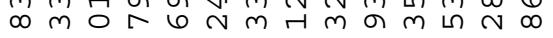
त

00000000000000

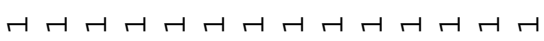

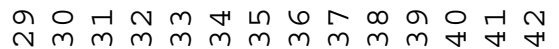

1 $N m 6+66 N$ \%

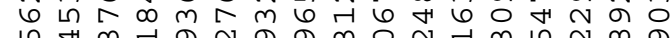

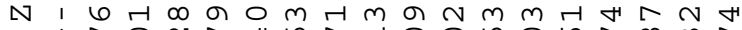

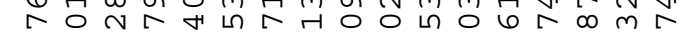
N

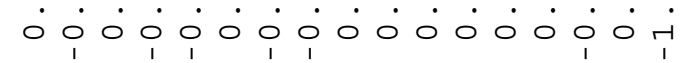

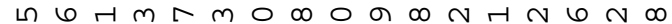
0 म ก

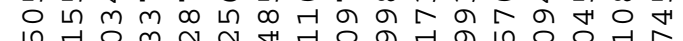
П $m \infty \pi m \infty$ म

ம

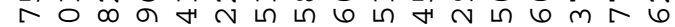

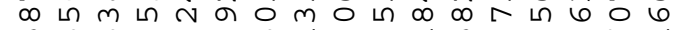

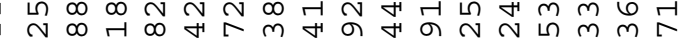

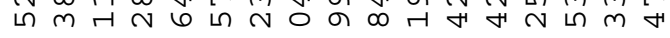

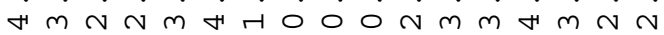

II

5

崖

O 1 U \& 
0 เ स

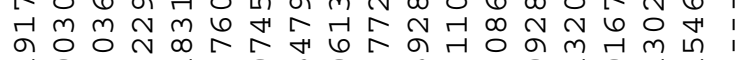
H $O N N$ H $N$ मी 0 m मी

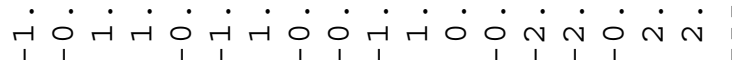

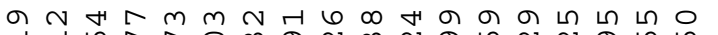
त

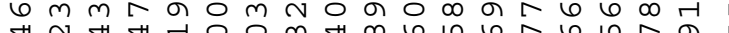
H $N$ H स $\infty \pi r+r \circ$ मी अ

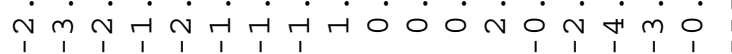

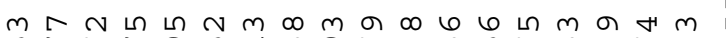

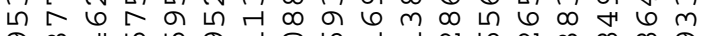
の $m$ म

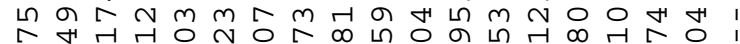

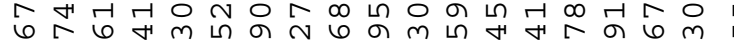

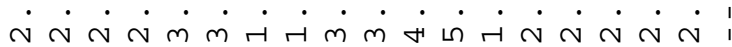

000000000000000000

6 6

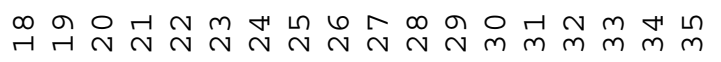

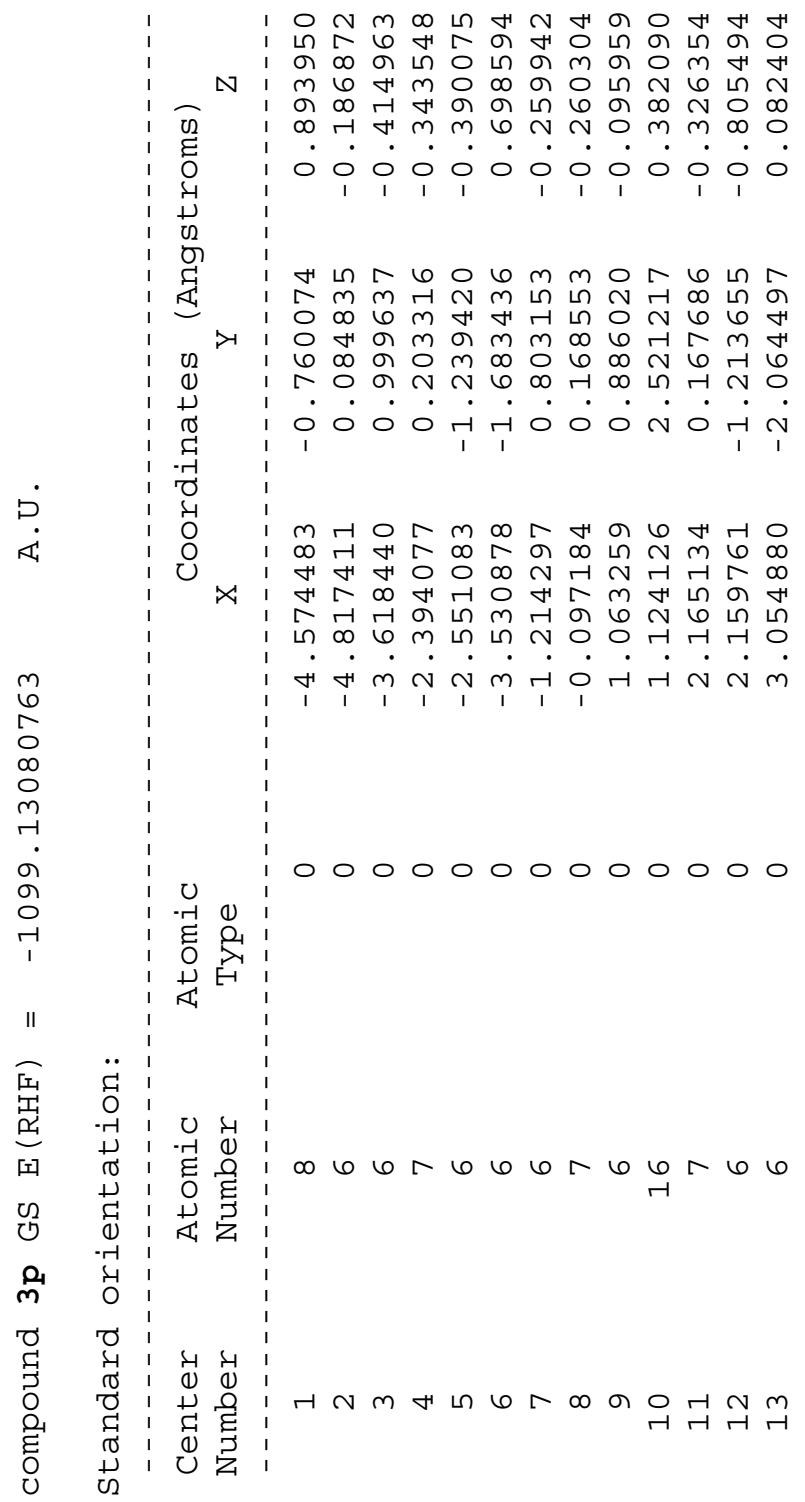


เ 6 \%

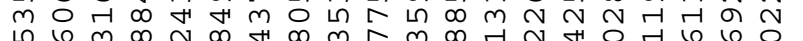

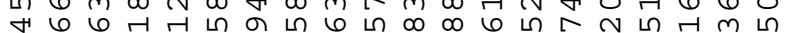

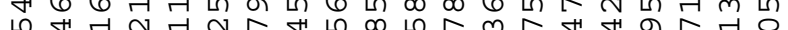

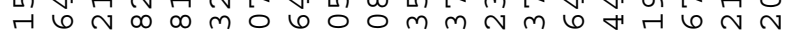

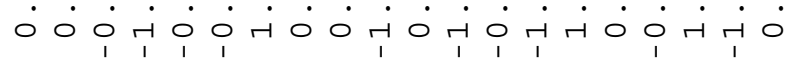

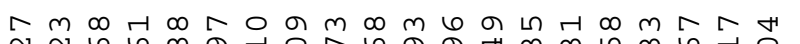
$\sim \sim 66 \infty$ ब H $\infty 6$ の

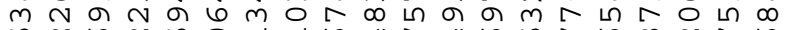

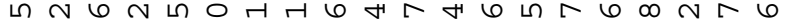
ت

अ

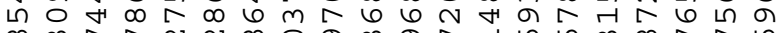

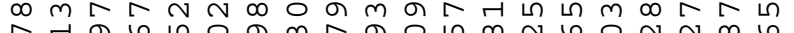
F $m m$ เ $\forall \dot{m} \sim \vec{m} \dot{m} \dot{\omega} \dot{m} \dot{m} \vec{m} \dot{m} \dot{m} \dot{m} \dot{m}$ 00000000000000000000 $\infty 6$

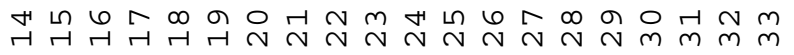

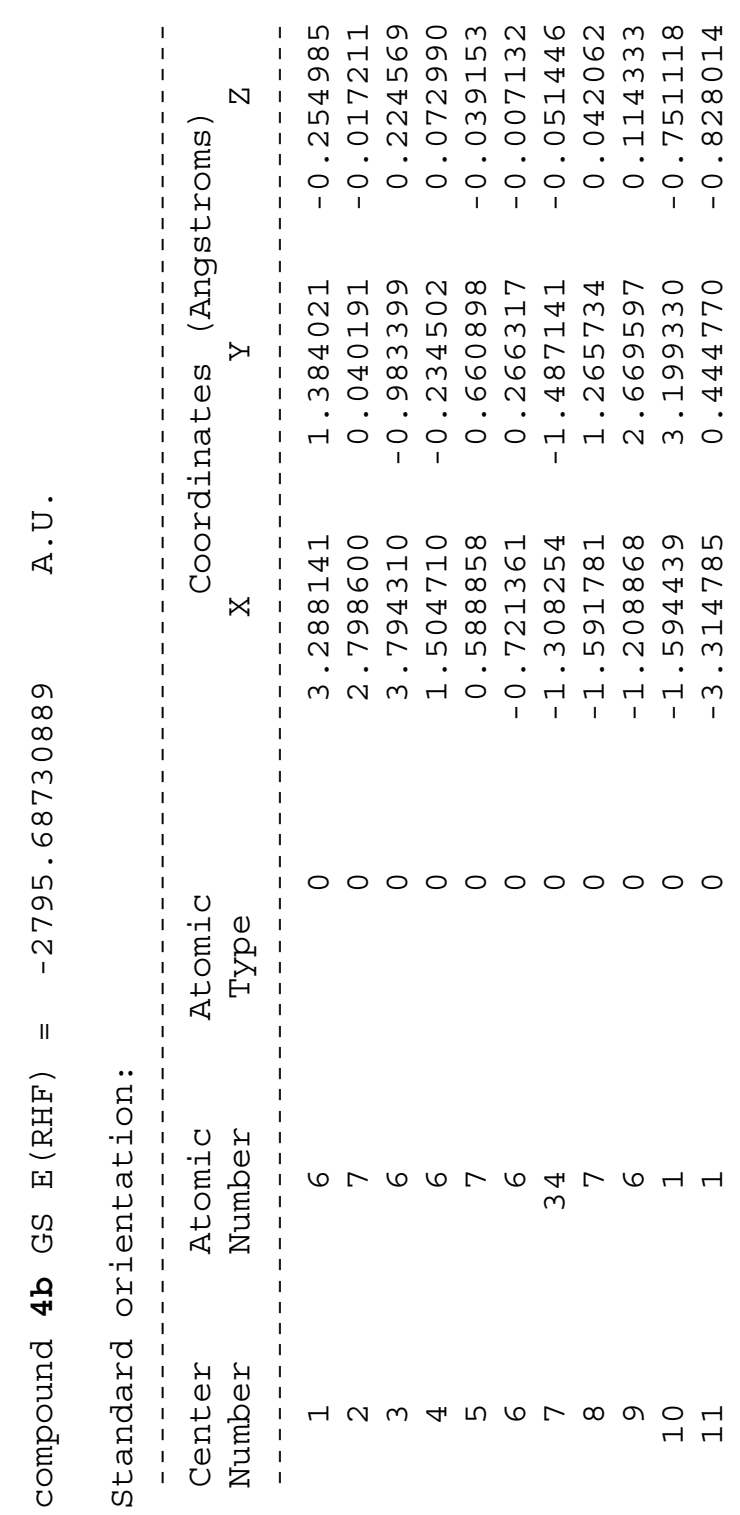


$\mathrm{N} N \mathrm{O}$ H 으대 각

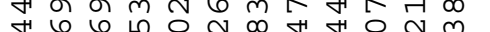
066 ब 6 $6 \mathrm{~m}$ त 0 ब 0

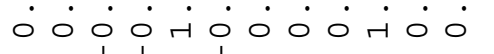

ब

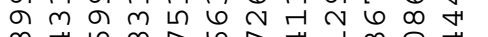
$\infty$ \& $\infty \curvearrowright 6 \wedge$ अ

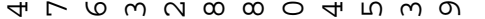

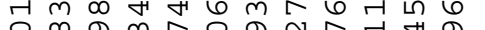
$0 \infty$ の $m$ の

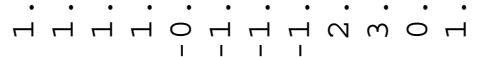

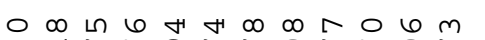
m

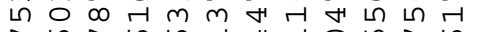
$\pi \quad \pi 66 \pi+40$ म $N m \sigma \sqcap \infty 6 \sqcap 6+m+m$

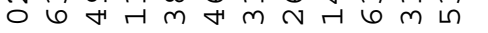

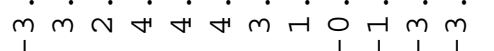

000000000000



त

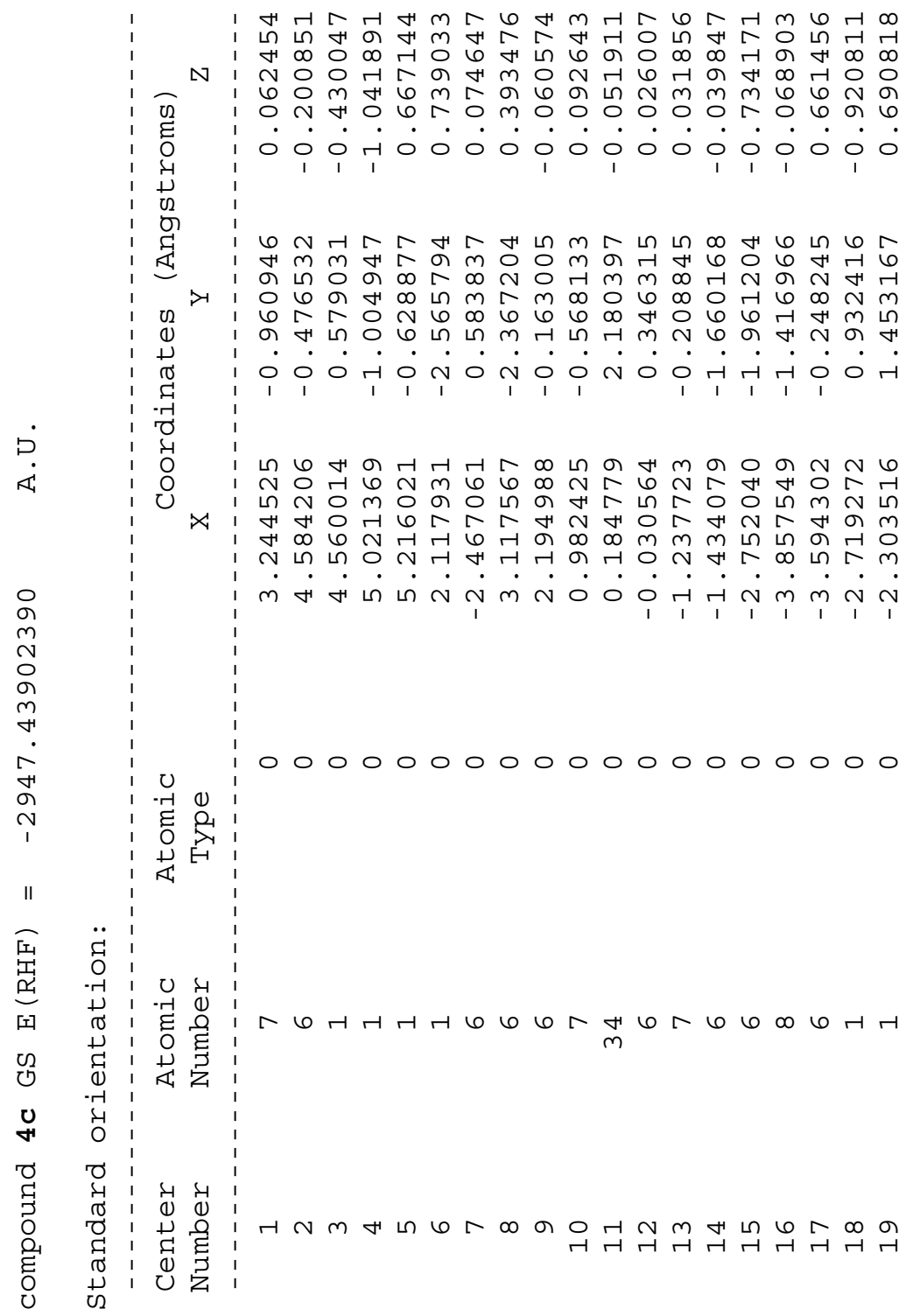

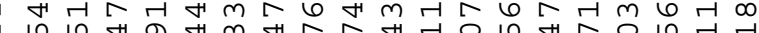
\&m $N \circ 0$ 다

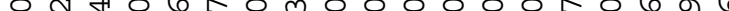

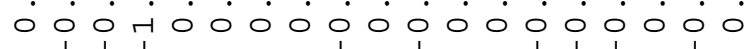

$6 N$ H

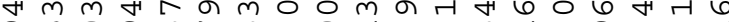
. 
N $N$ H

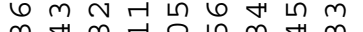

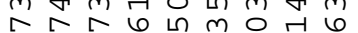

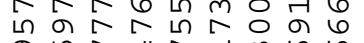

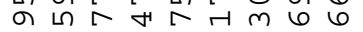

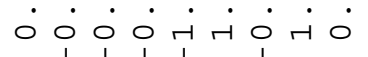

A 0 4 मे $N$ त $\infty$ ब

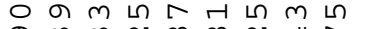
の $m m \sim \infty \infty \sim$ $\infty \circ m \infty \infty \sqcap \omega \circ m$

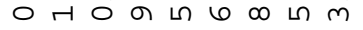

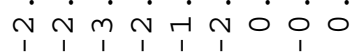

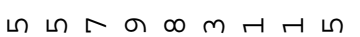
ब $N$ म $\infty \circ \infty \sim$ $\sim \sim N \sim N \sim ⿻$ ง $\forall 6 \infty m-\infty \quad+\infty$ $\vec{H}_{1} \dot{0}_{1} \dot{N} \dot{m} \dot{m} \dot{N} \dot{m} \dot{1}$

000000000

म

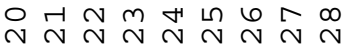

I ก

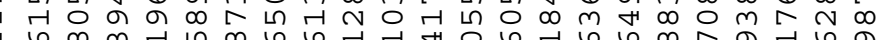

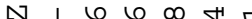

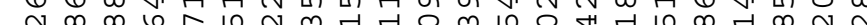
००

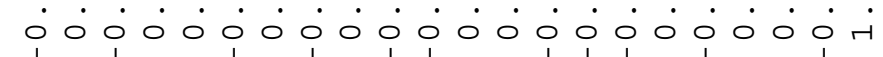

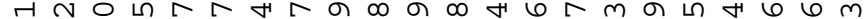
स

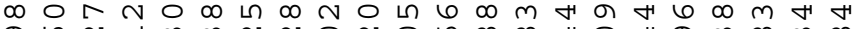

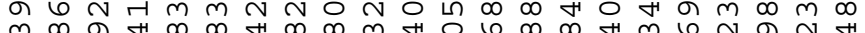

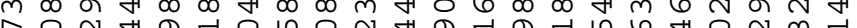

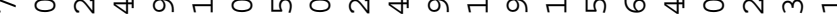

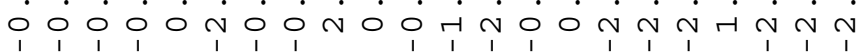

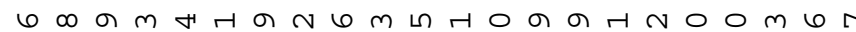
त म $n$
$\pi$ ○ $\infty$ ब

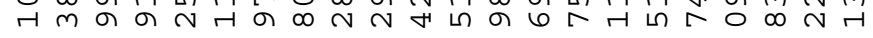

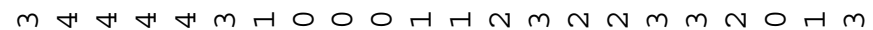

II

ב:

U

0000000000000000000000 है 


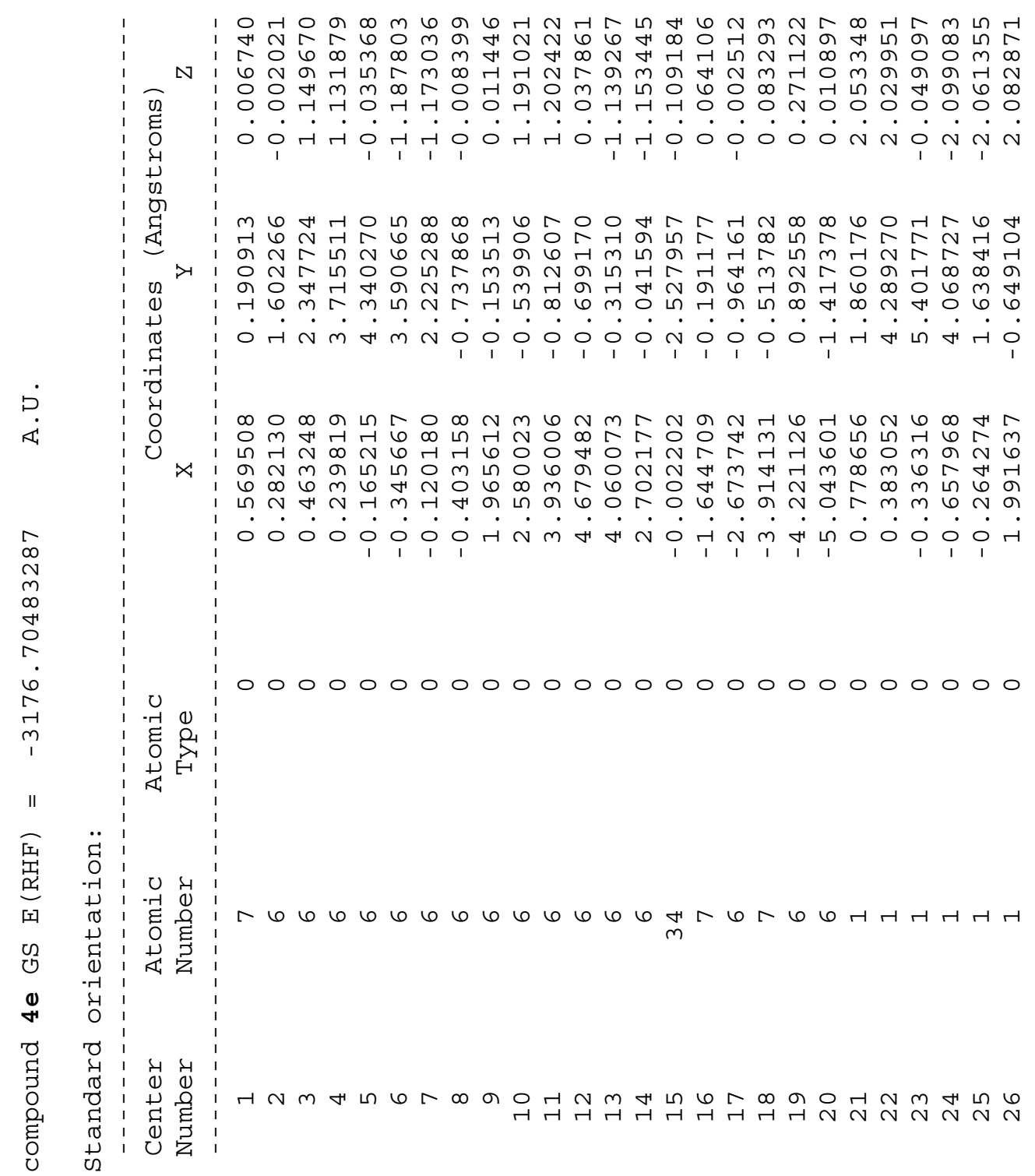




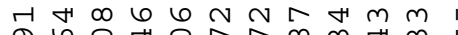

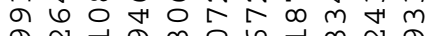
ब $N$ त म $+6 \mathrm{~m} m \sim$ N $N$ N

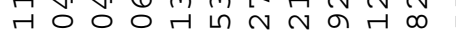

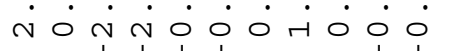

L $\infty$ L 4 4 स

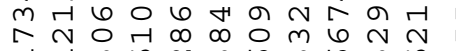
H $\mathrm{m}$ เ $N \mathrm{~m} 6 \mathrm{~m} 6 \mathrm{~m}$ ก H

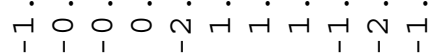

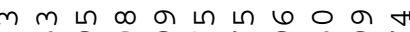
$N$ ட $○$ の 6 の

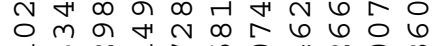

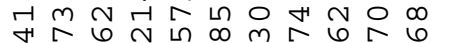

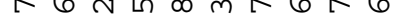
म 00000000000 म 4 म 4 म 4

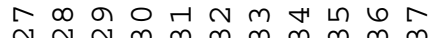

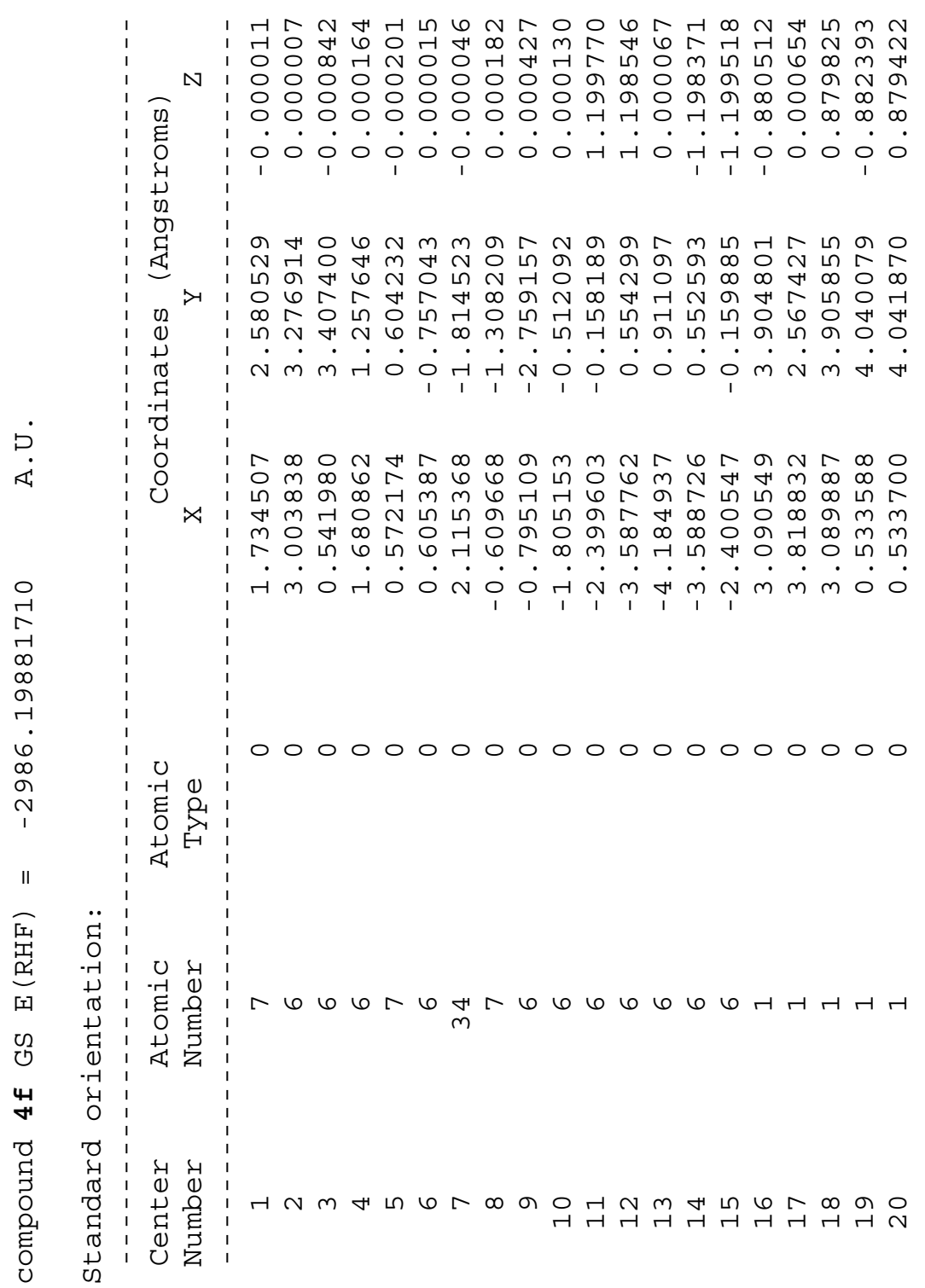


बतनONTHa H 6
L $N$ 당

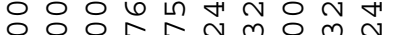
000000 म 00 H

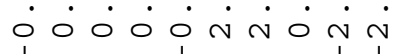

$6 \infty 4+4 m N \hat{m}$ त $00 \infty 0$ त 0 の 6 の $6 \mathrm{~m} m$ 에

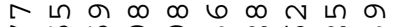
$\sim$ ก $\curvearrowleft$ の $m \sim 6 \sim m$ ก

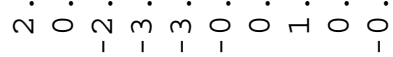

$\infty 6 m \infty \circ 6$ \% 6 ம $m$ の 几 न बि कित

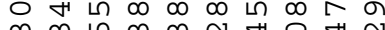

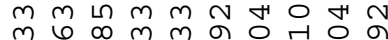

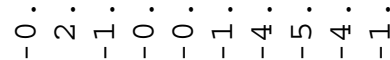
0000000000 $444-44-44$

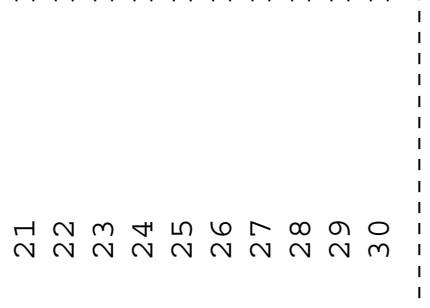

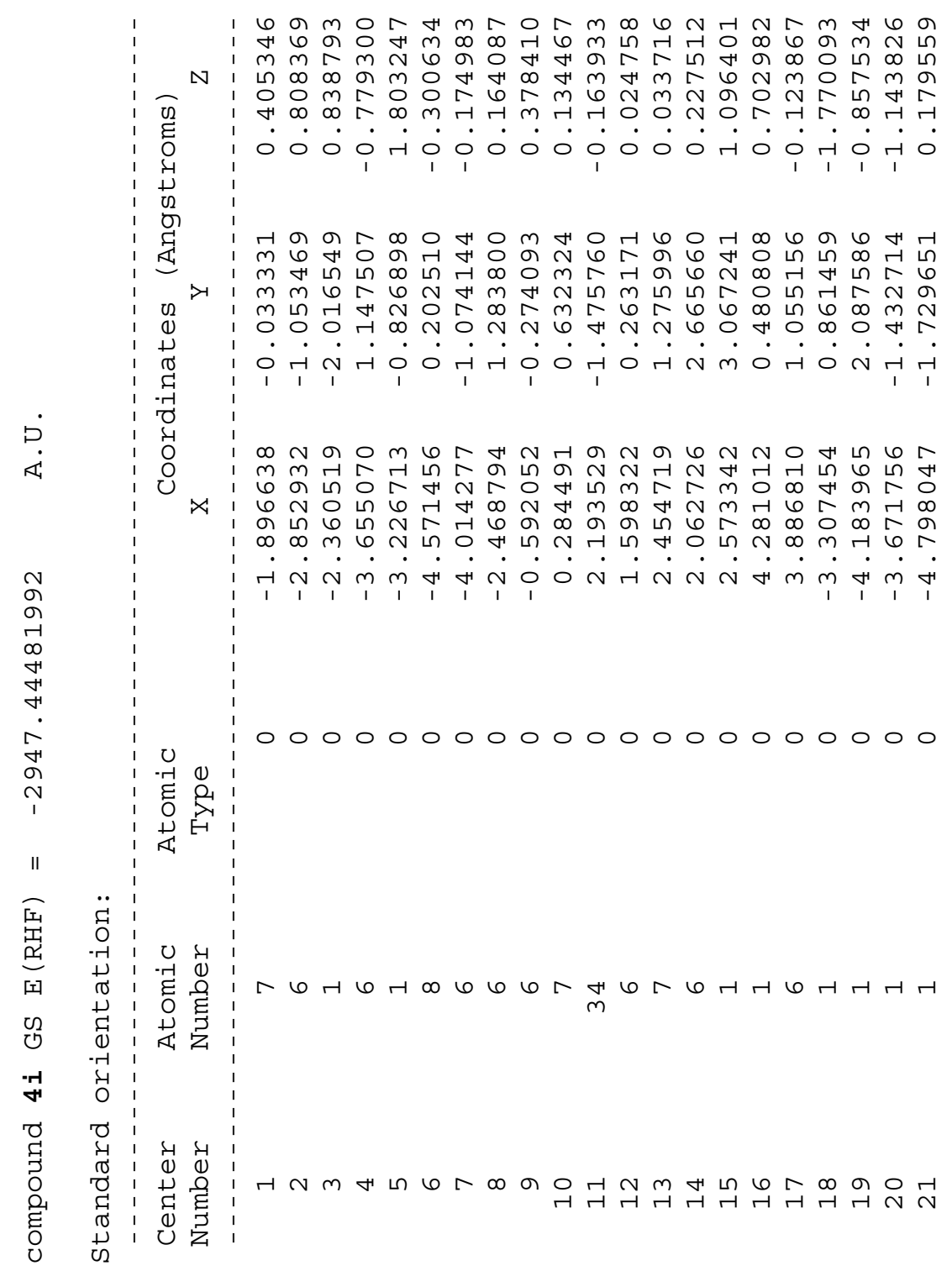

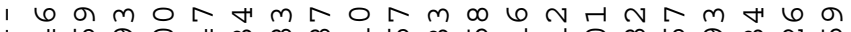

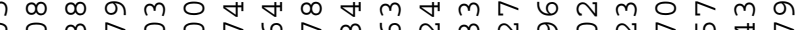

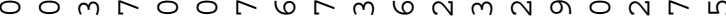

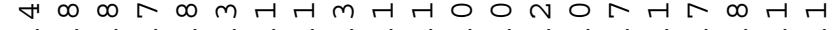

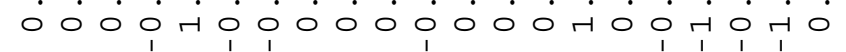

- $20 \infty 00 \%$ ( ( 西 $\begin{array}{lll}1 & 1 \\ 0 & 1 & 1\end{array}$

๓

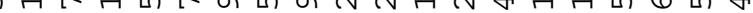

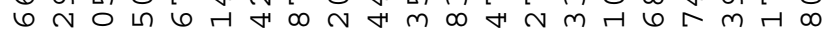

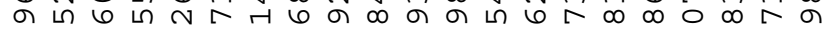

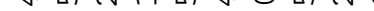




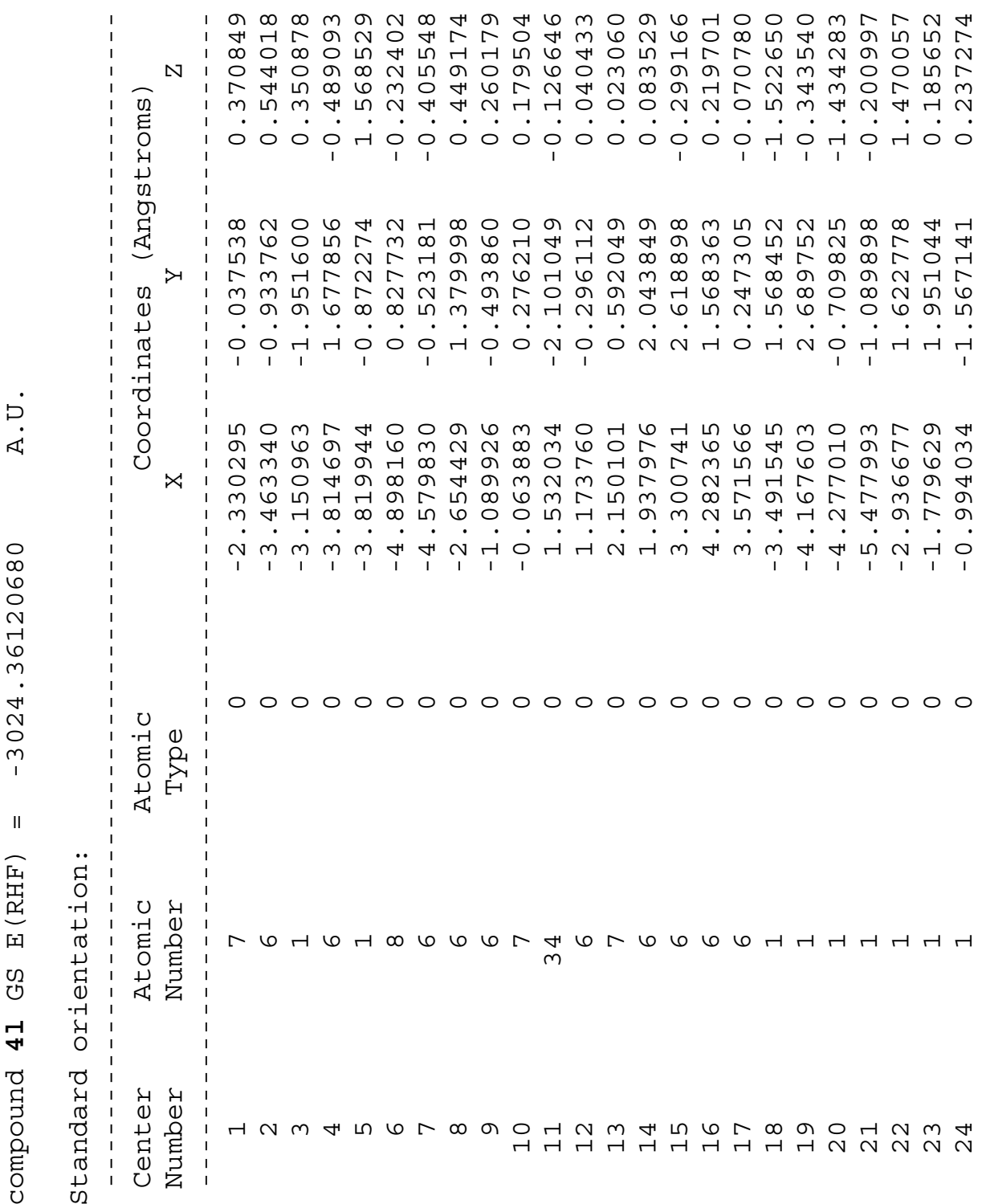


$40 \infty N \circ \pi$ a

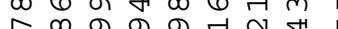

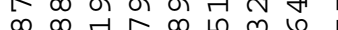
0 เ $\mathrm{m} N \mathrm{~N} 0$ में 0 में

$m \infty a \circ+m \circ$ เ $\forall m \infty \forall \forall M N$ $m\llcorner N \sim \infty \pi n$

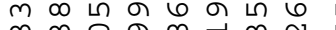
$m m \circ \sigma \infty r m \sim$

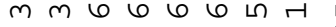
$\dot{\sim} \dot{m} \dot{\sim} \dot{H} \dot{r} \dot{0} 0$

$\circ 00 n \infty$

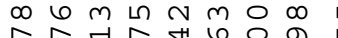
†ิ

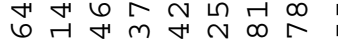
तं तं $\dot{m} \dot{m} \dot{\forall} \dot{m} \dot{m}$

00000000

$44-44-44$

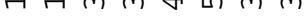
○ - $ब \infty 6000 \%$

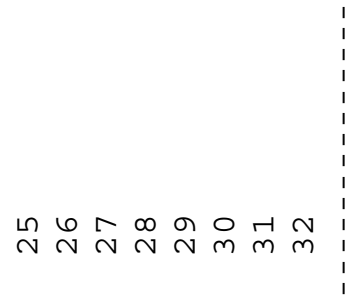

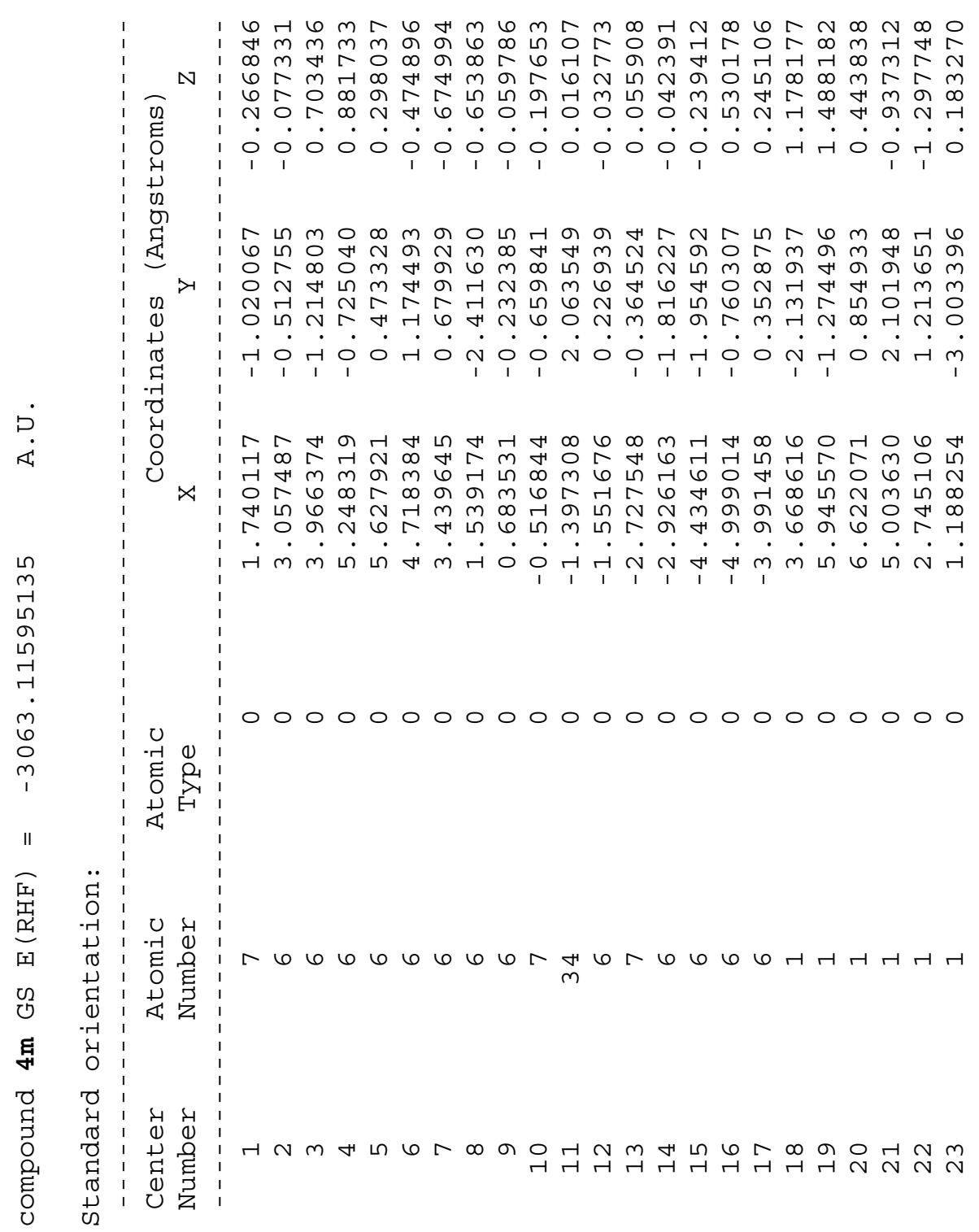

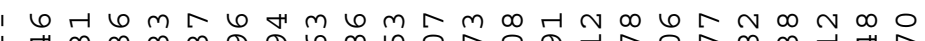

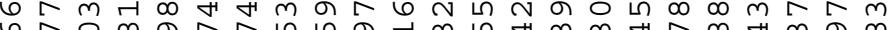

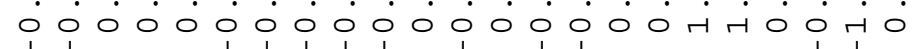

न

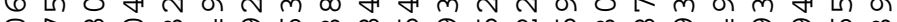
年

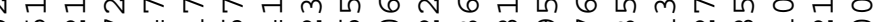

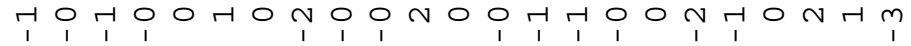

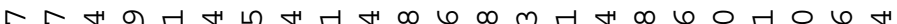
每 स 人 0 の 6 H

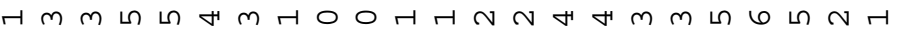




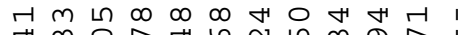
H $m$ O $\pi 0 N N$ N H $4 \hat{4}$ in 0 H $\infty \infty 4 N, \infty$ تं

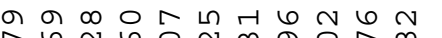

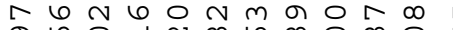
の 10 ก 0 N $\infty$ \& $N$ N $\dot{\sim} \dot{0} \dot{\sim} \dot{\sim} \dot{\sim} \dot{0} \dot{0} \dot{0} \dot{0}$

ก 0 H

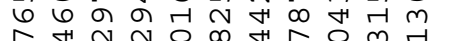

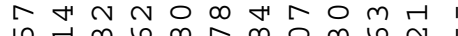

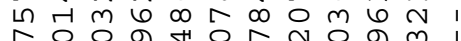

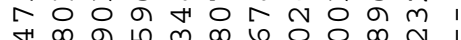
अ $\infty$ न म $m \infty 600 \infty \mathrm{N}$

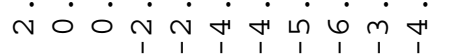

00000000000

Н

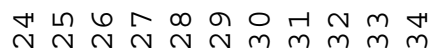

$m$ ㄷ の $\infty$ r त० $N, \infty \infty \infty, \infty)$

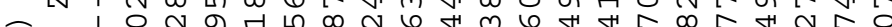
U I $m N$ N $\infty$ H 0 FN

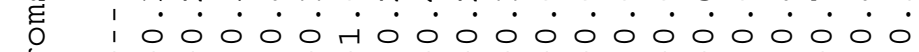
(1)

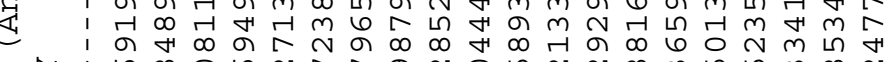

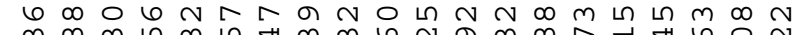

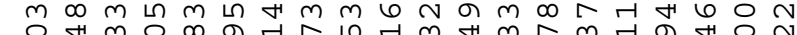
0 म 0 ब न ن

H $\infty$ เ 6 เ

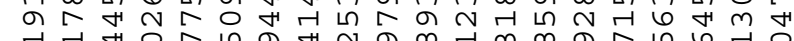
1

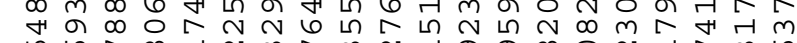

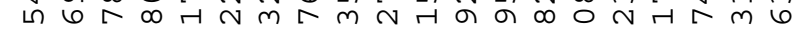

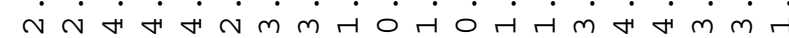




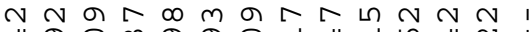
स ब $0 \mathrm{~m}$ ब व 0 म न 1 मी

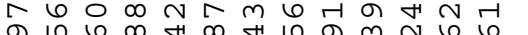
$6 \curvearrowright 66$ ㄴ $\infty$ ब $N$ त $\mathrm{m}$

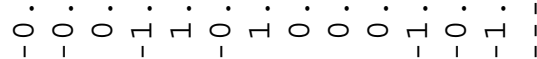

ก I m

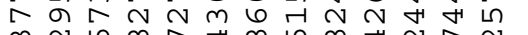

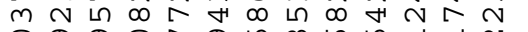

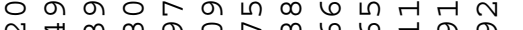

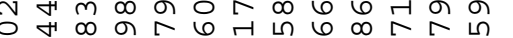

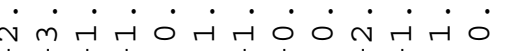

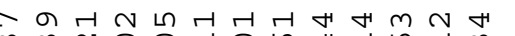
$m m N O 0$ H 06 H

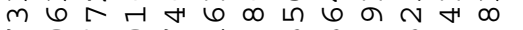

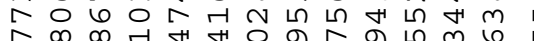
बे ब

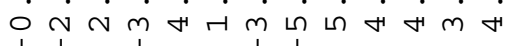
0000000000000 44444444444

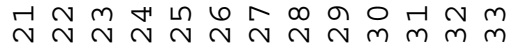

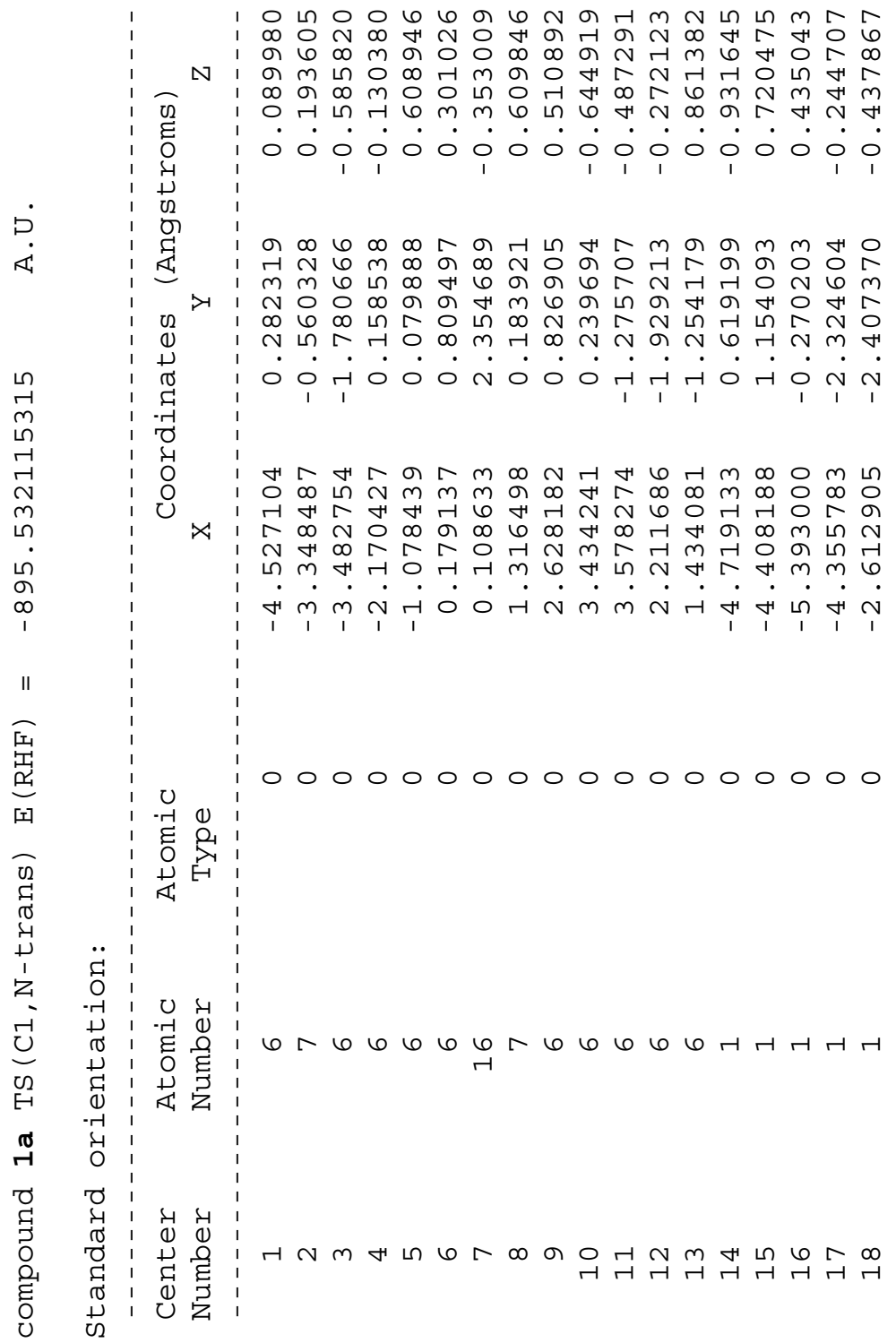


$m$ 다 $m$ 대 6 म $m \circ$ व

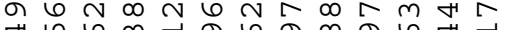
अ

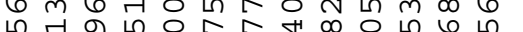
60 म त

No $\circ 006$ ब $\sim$ म 그

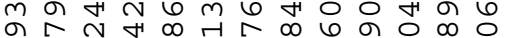

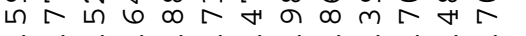

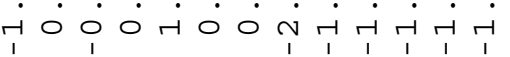

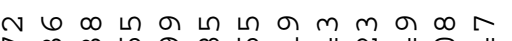

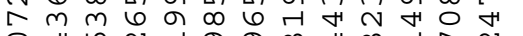
○ म $\mathrm{N}$ न

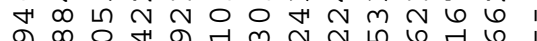

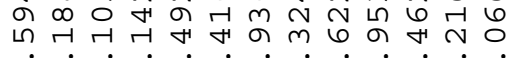

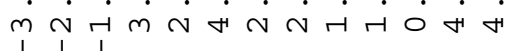
0000000000000

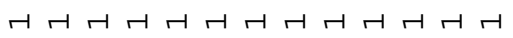

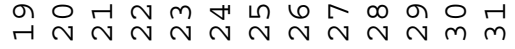

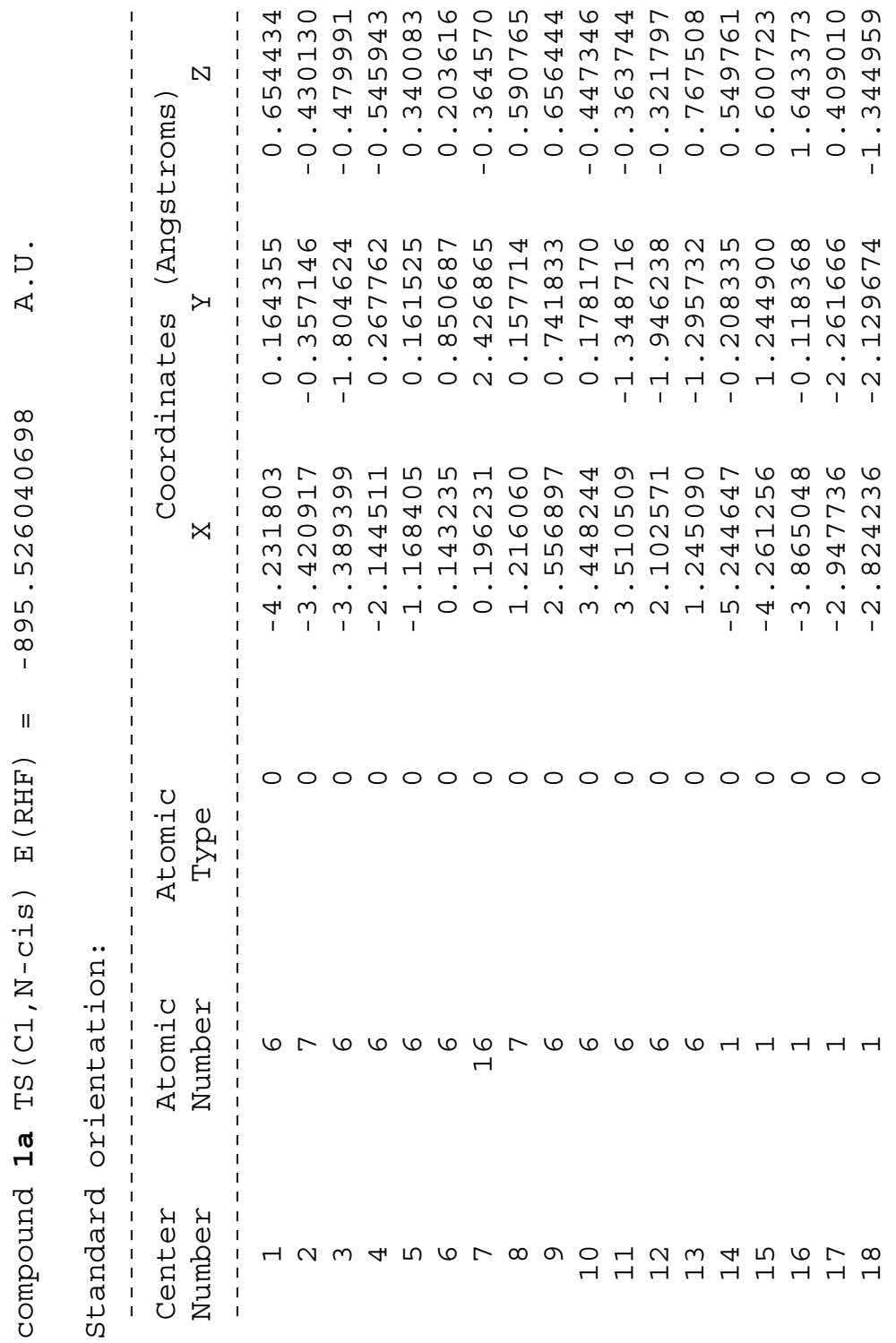


त

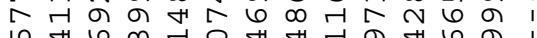
பी

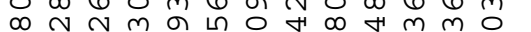

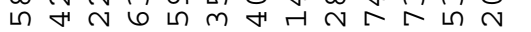

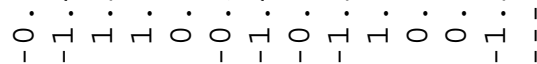

Hก $ก$ ก $\infty \kappa \sigma+m m$ ก

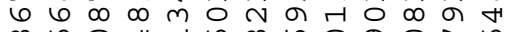
$\infty$ L 0 म

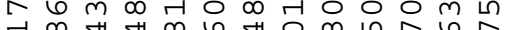

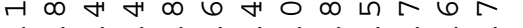

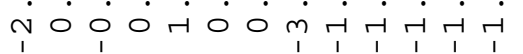

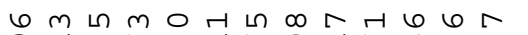

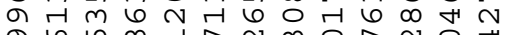

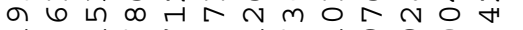

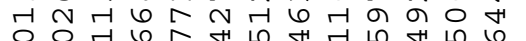
म० मi $\dot{\sim} \dot{\sim} \dot{\sim} \dot{m} \dot{n} \vec{r} \dot{0} \dot{4}$ 0000000000000

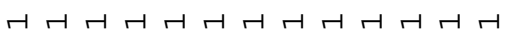

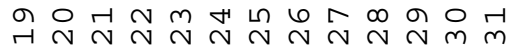

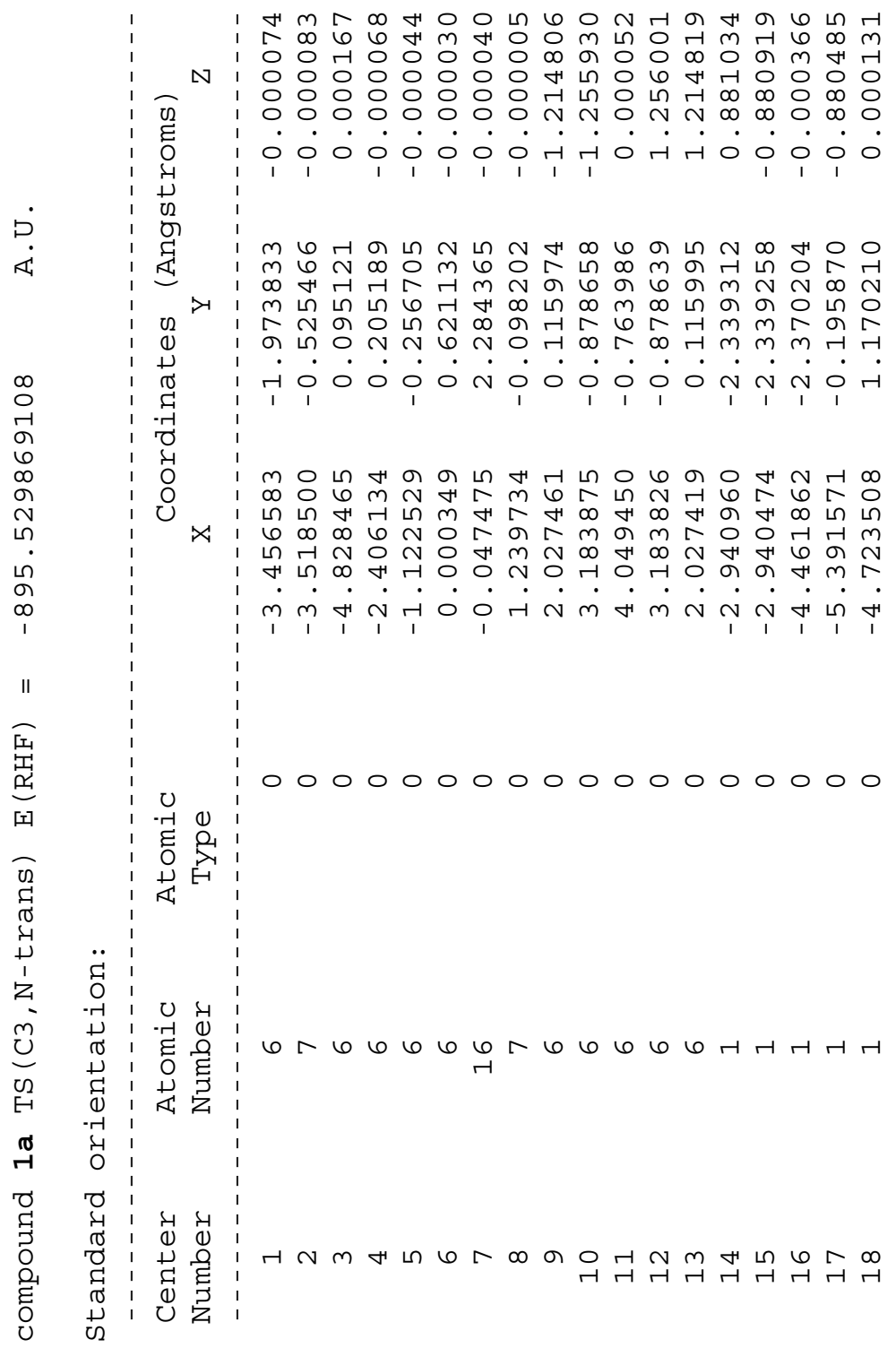


$\infty 6$ เ $\infty$ ब

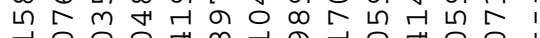

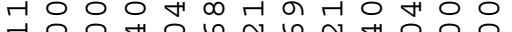

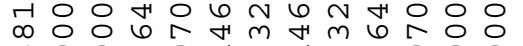
$\infty$ O $0 N 0$ H $\mathrm{M} M N 000$ $\dot{0} \dot{0} \dot{H} \dot{N} \dot{\sim} \dot{\sim} \dot{H} \dot{H} \dot{0} \dot{0}$

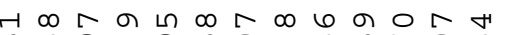

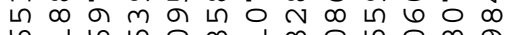

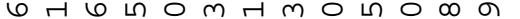

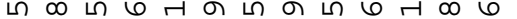
の $6 \circ m m \sigma \infty \sigma \infty m m \sigma N$

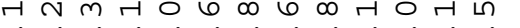
○ म

स ए $6 \curvearrowright \infty 6 \%$ म $\pi N m m m \infty N$ न่

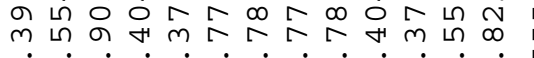
ن 0000000000000 44444444444

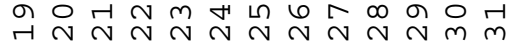

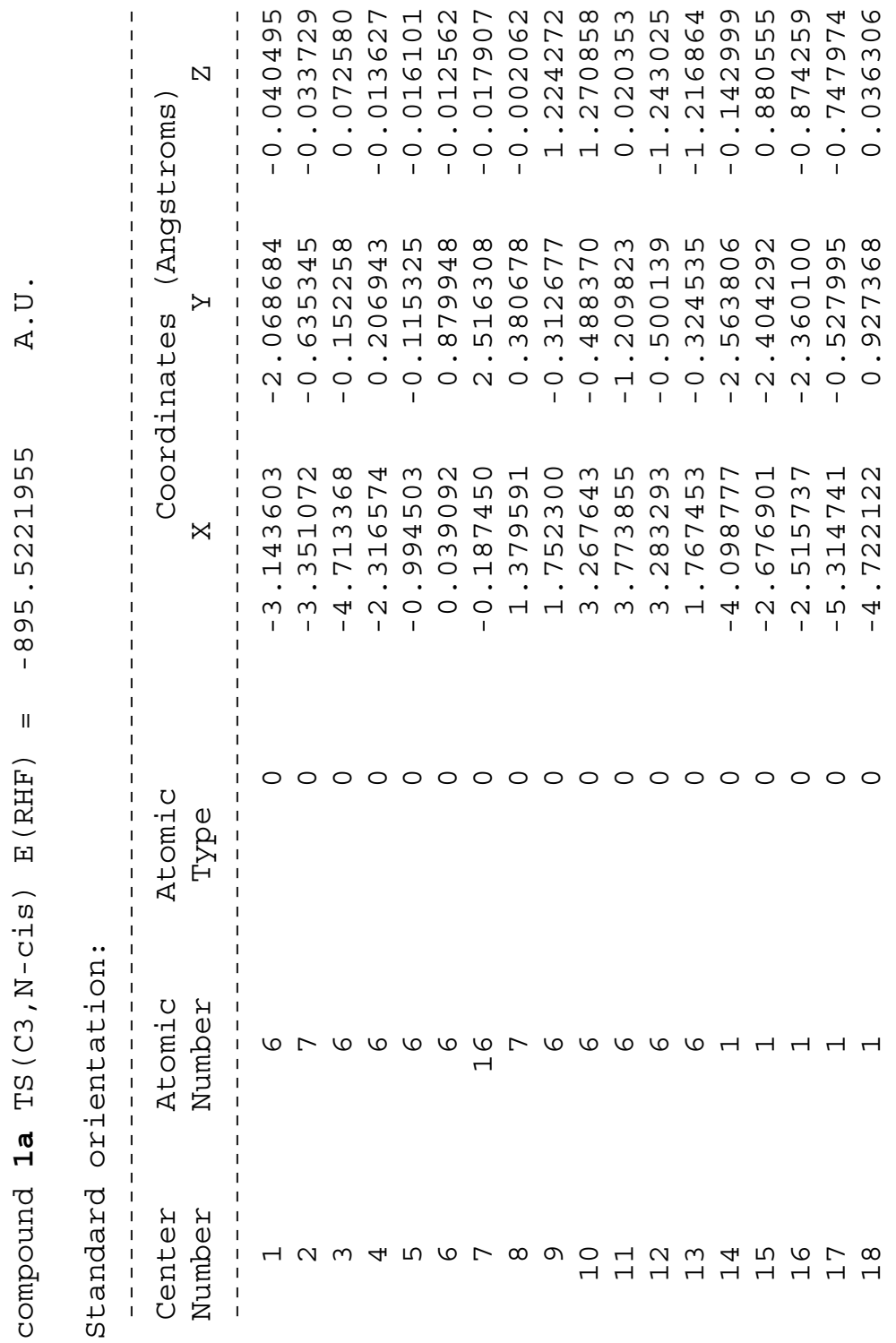




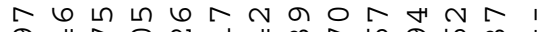
ब HN $N \in N$ H

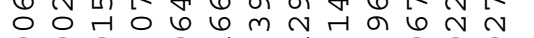
$000 \mathrm{~m} 0 \mathrm{~m} M \mathrm{~m} N 000$

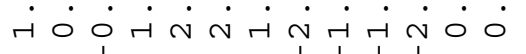

人

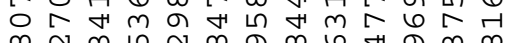

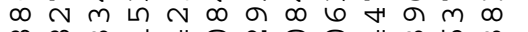
$\infty \quad \infty m$ r 6 अ A N

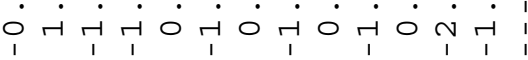

$\infty 6$ ก เ

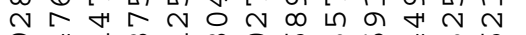
ब मत ๘ก

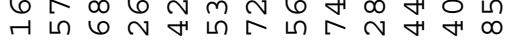
$\dot{0} \dot{0} \dot{0} \dot{m} \dot{m} \dot{m} \dot{r} \dot{r} \dot{m} \dot{~}$ 0000000000000

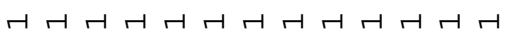

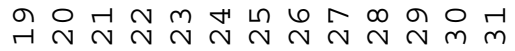

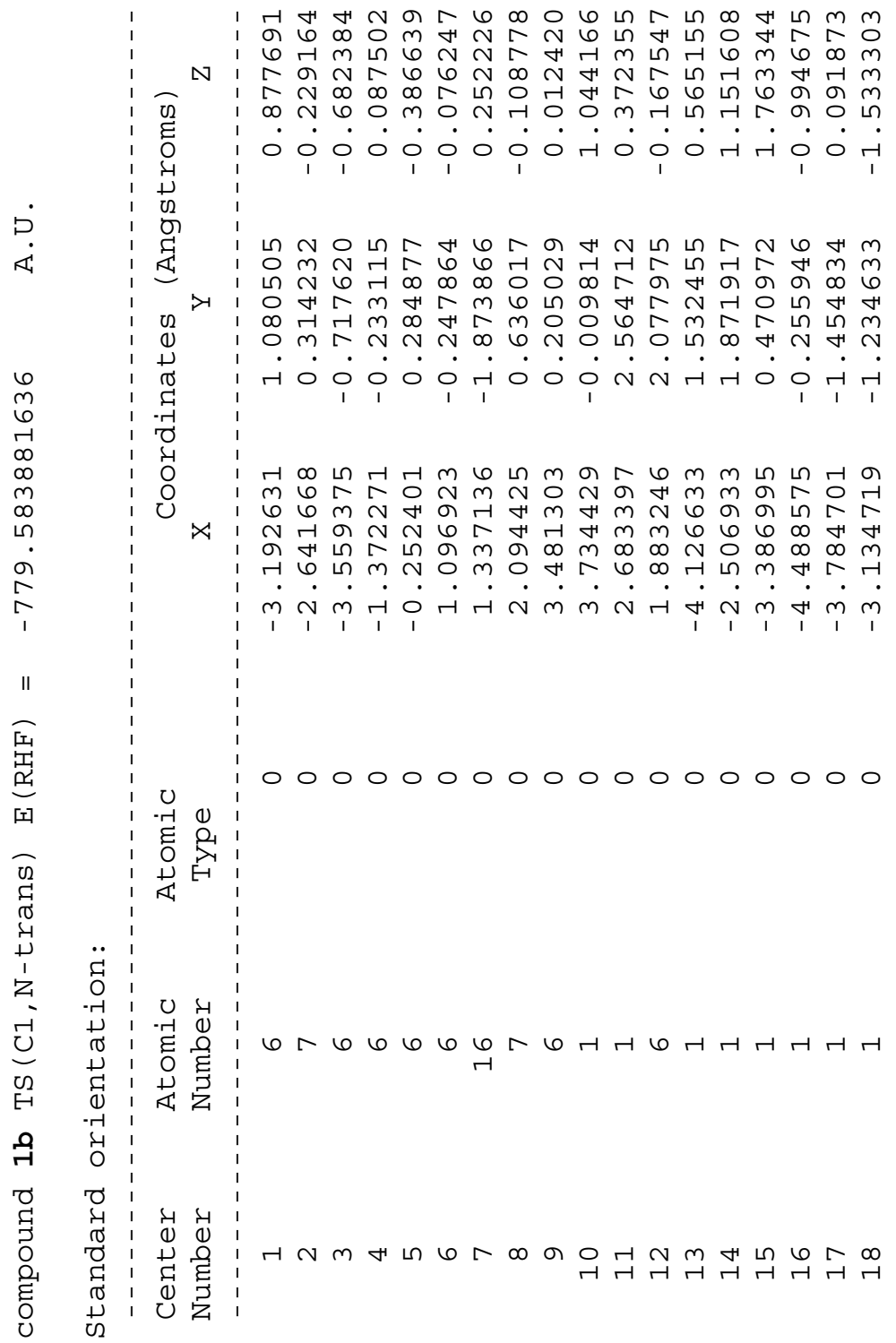




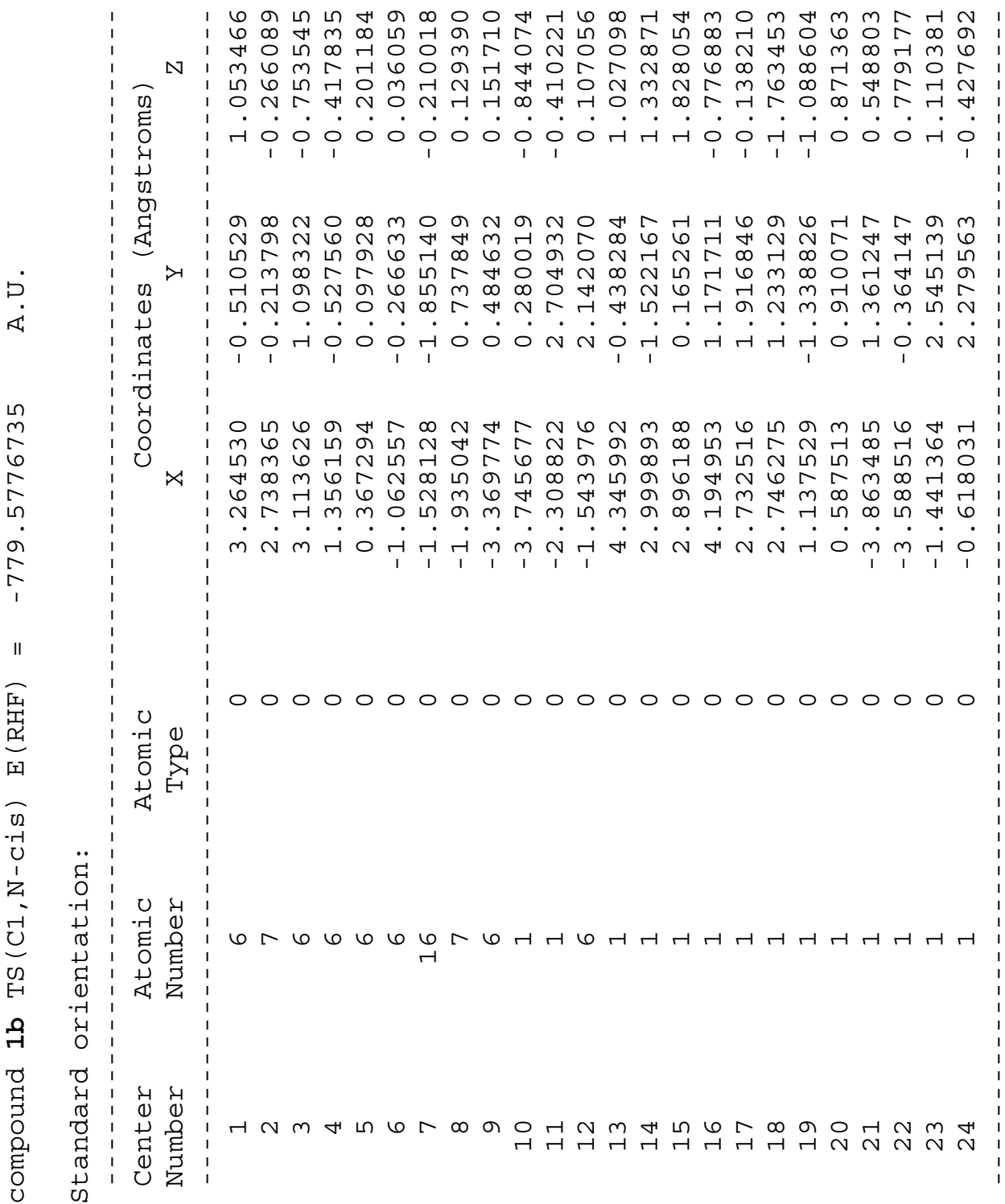




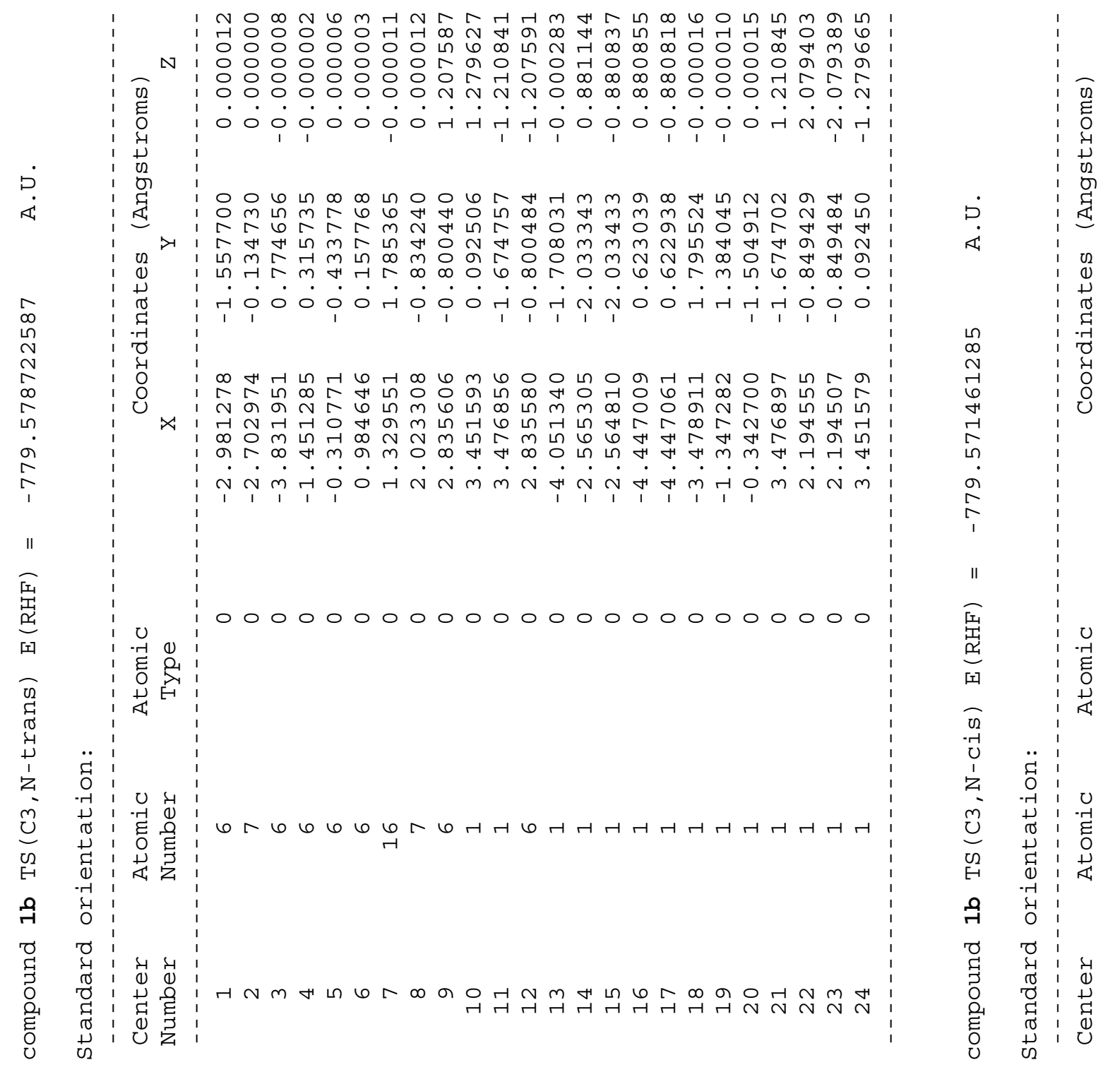


06 H

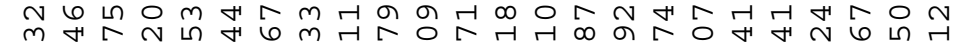

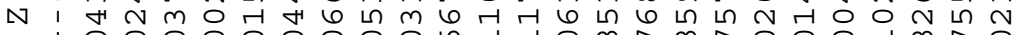

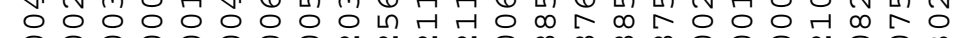

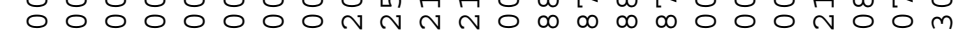
$\dot{0} \dot{0} \dot{0} \dot{0} \dot{0} \dot{0} \dot{0} \dot{1} \dot{1} \dot{0} \dot{0} \dot{0} \dot{0} \dot{0} \dot{0} \dot{0} \dot{0} \dot{0}$

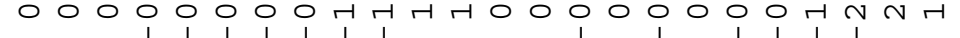

H⿻上丨 0 เ $\ln$ H

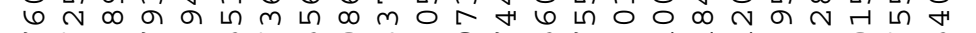

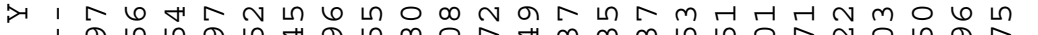
G เก

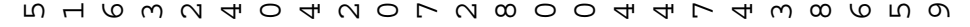

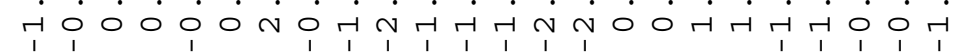

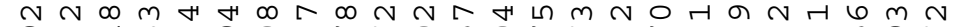
ब $N$ H $\infty$ स 0 म

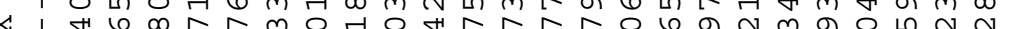
i

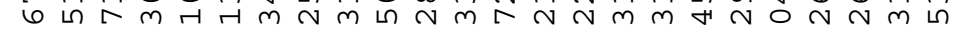

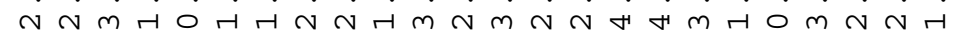

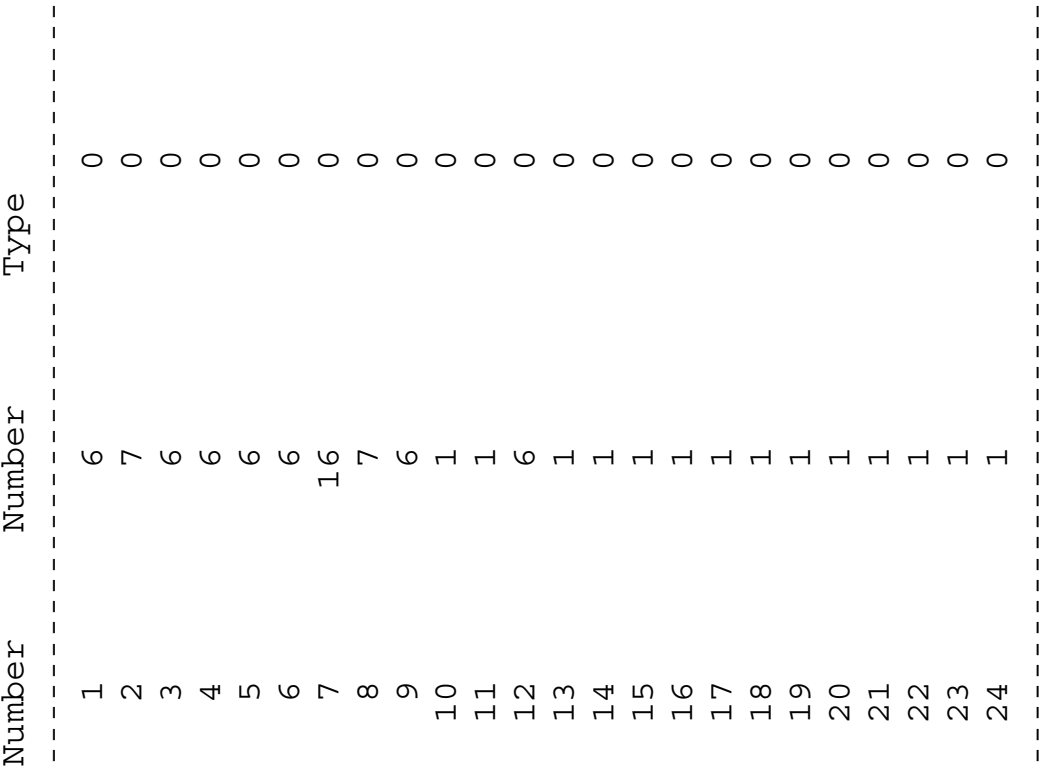

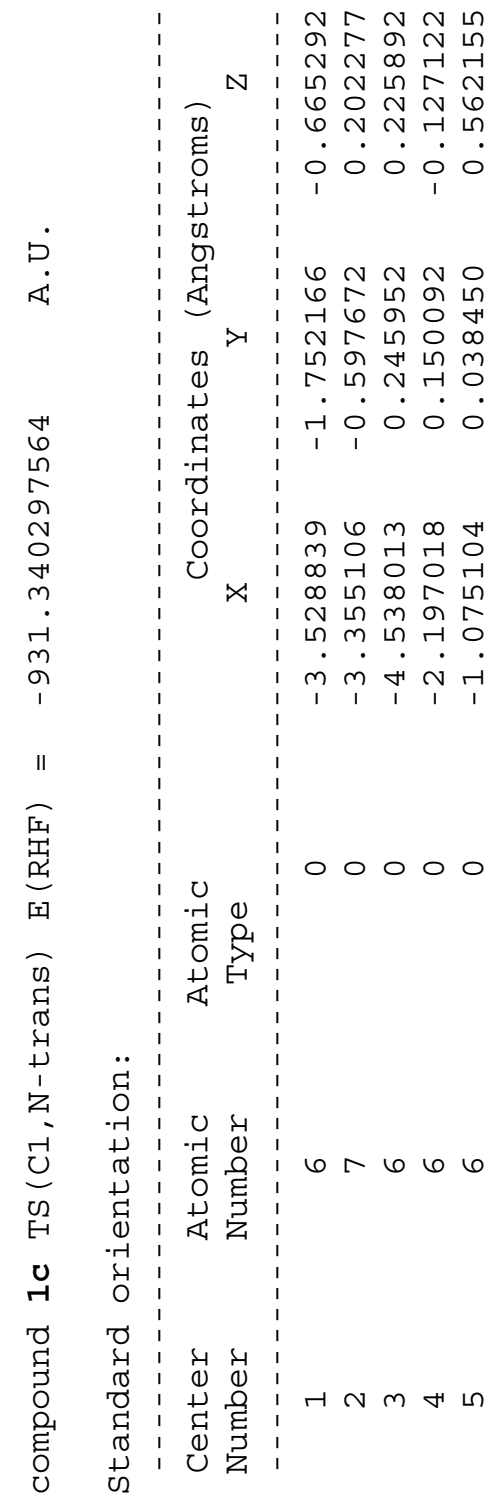




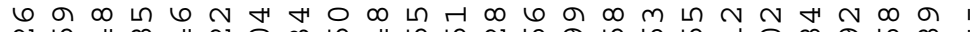
$N$ N 岁 अ $m$ の N $m$ H

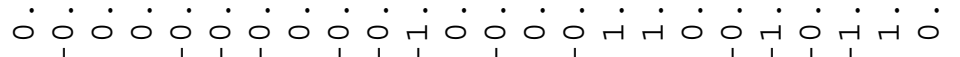

(4) H

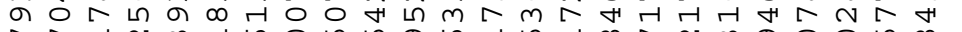

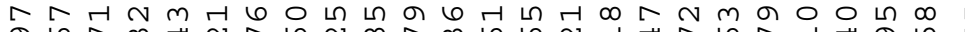

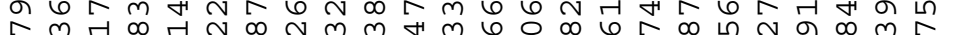

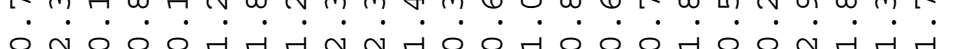

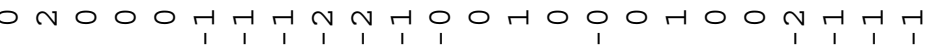

ए

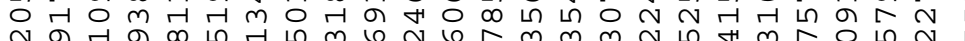
$N$ ब

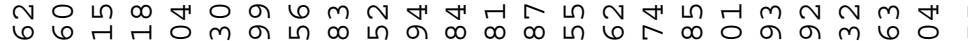

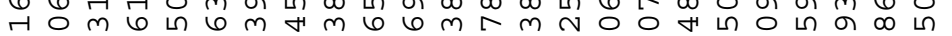

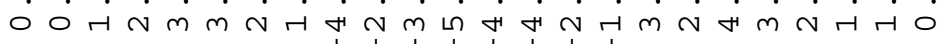

000000000000000000000000

6

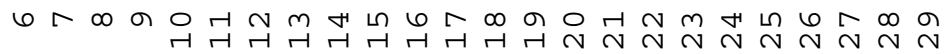

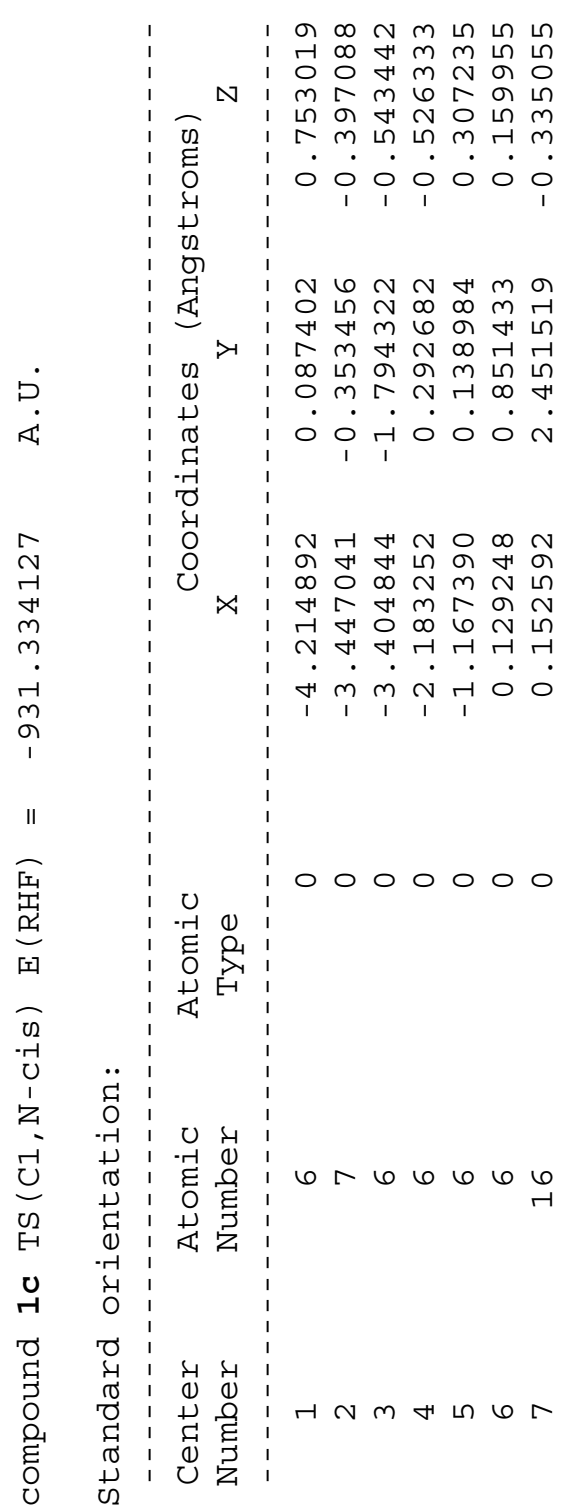




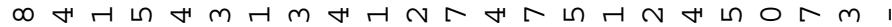

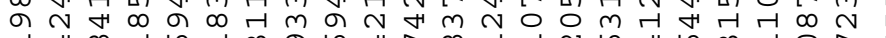

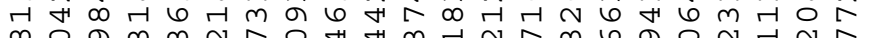

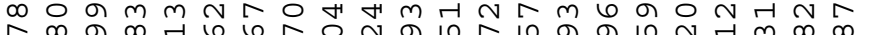

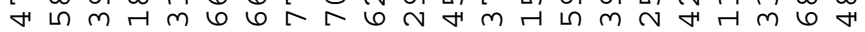

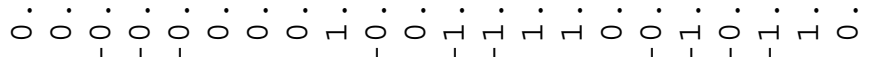

06 の

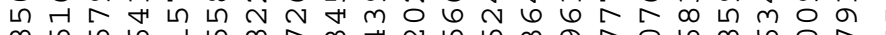

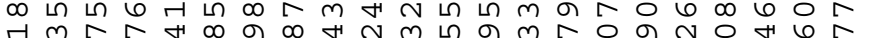
ए

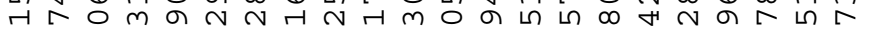

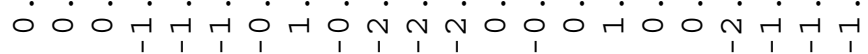

の $\mathrm{Nm}$ ต

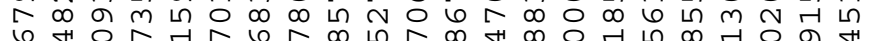

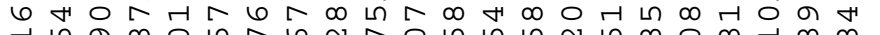
간

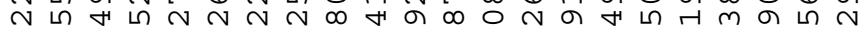

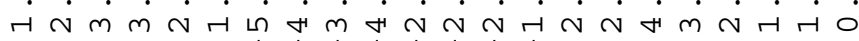
0000000000000000000000 $\lambda$.

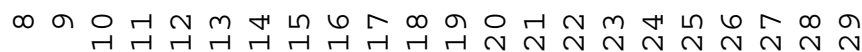

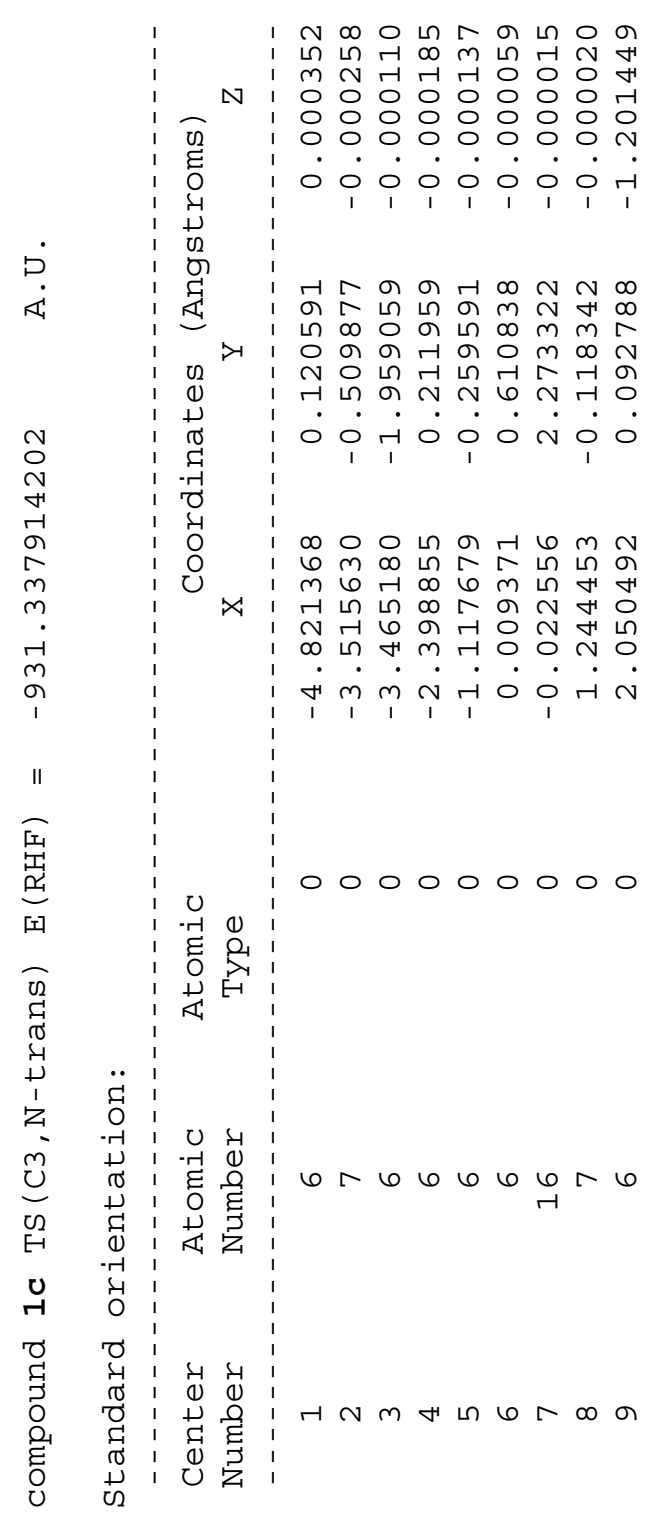


त

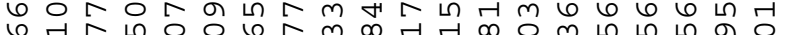

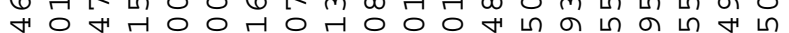

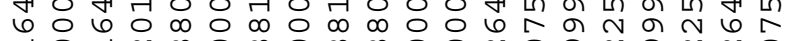

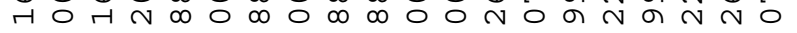

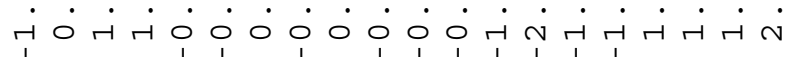

$\circ$ เ

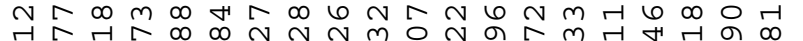

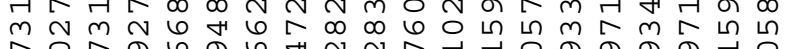

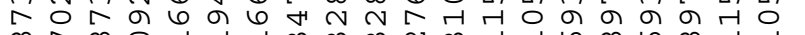

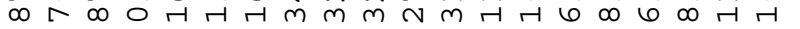

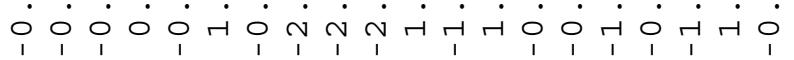

H $\infty m m$ h ก $N$ O

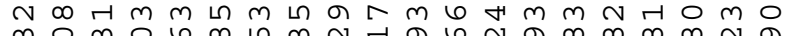

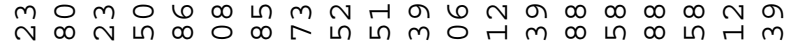

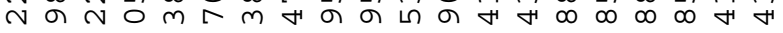

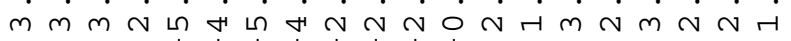
00000000000000000000 e 6 ש

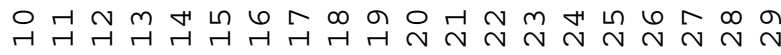

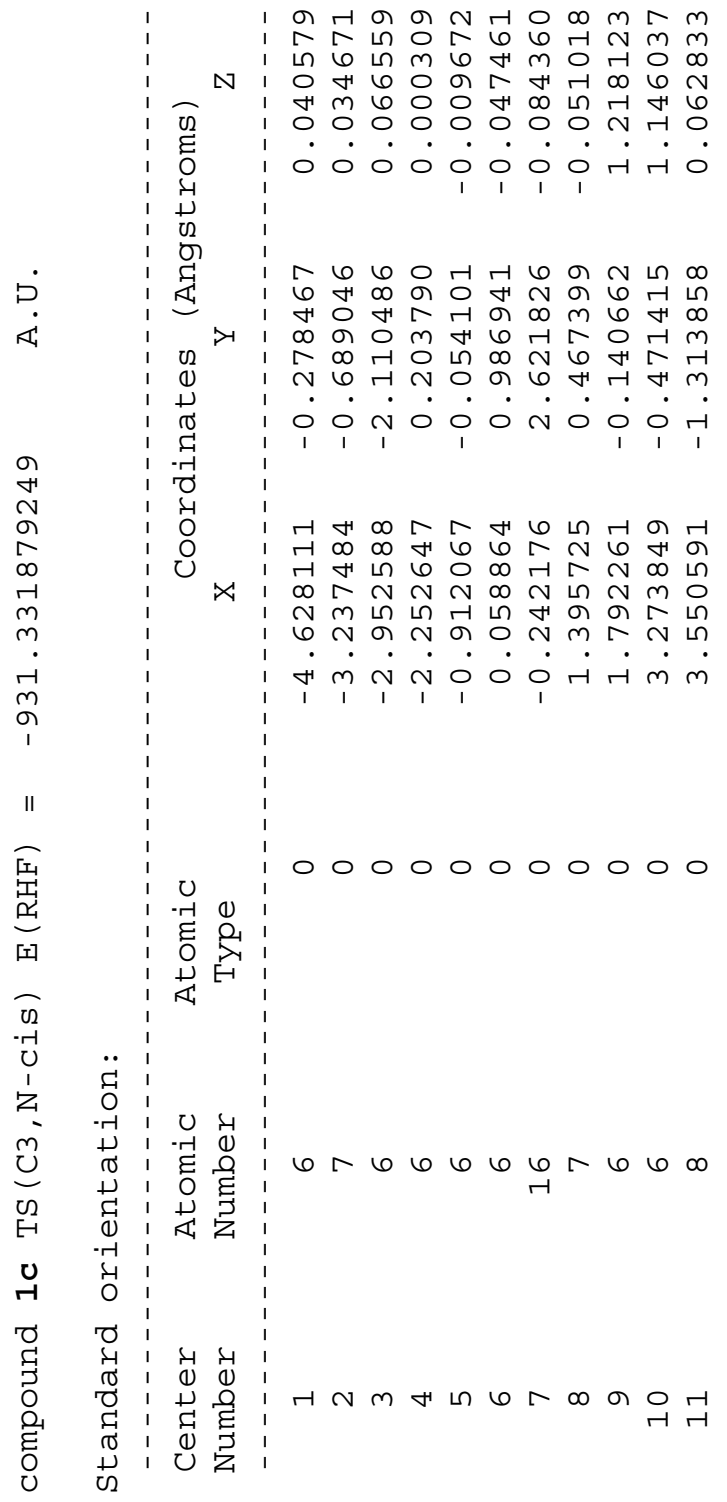


$m \varphi \propto \varphi \varphi 6 \circ N \infty m \circ \infty \Gamma \sim m \varphi m b$ ।

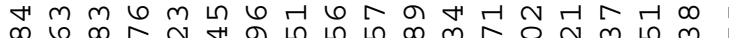

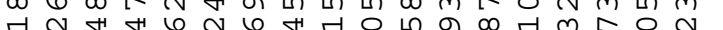

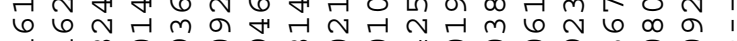
त

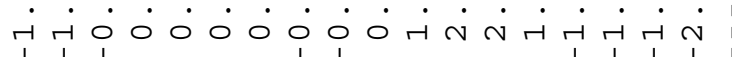

$m$ の $m \infty m$ 다

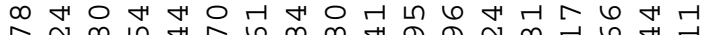
$[N \infty$ เ

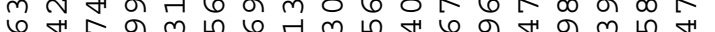

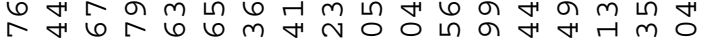

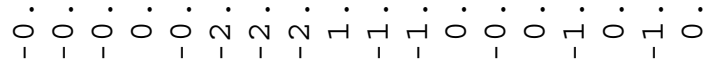

$m 6 m$ m 100 a $m 00$ a

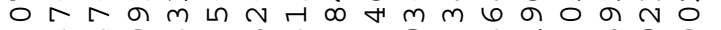

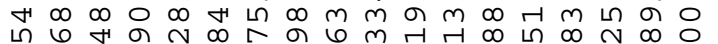

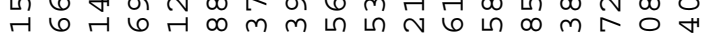

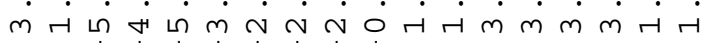
000000000000000000 6

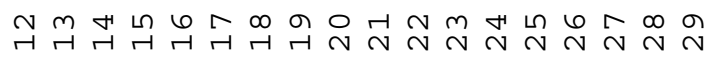

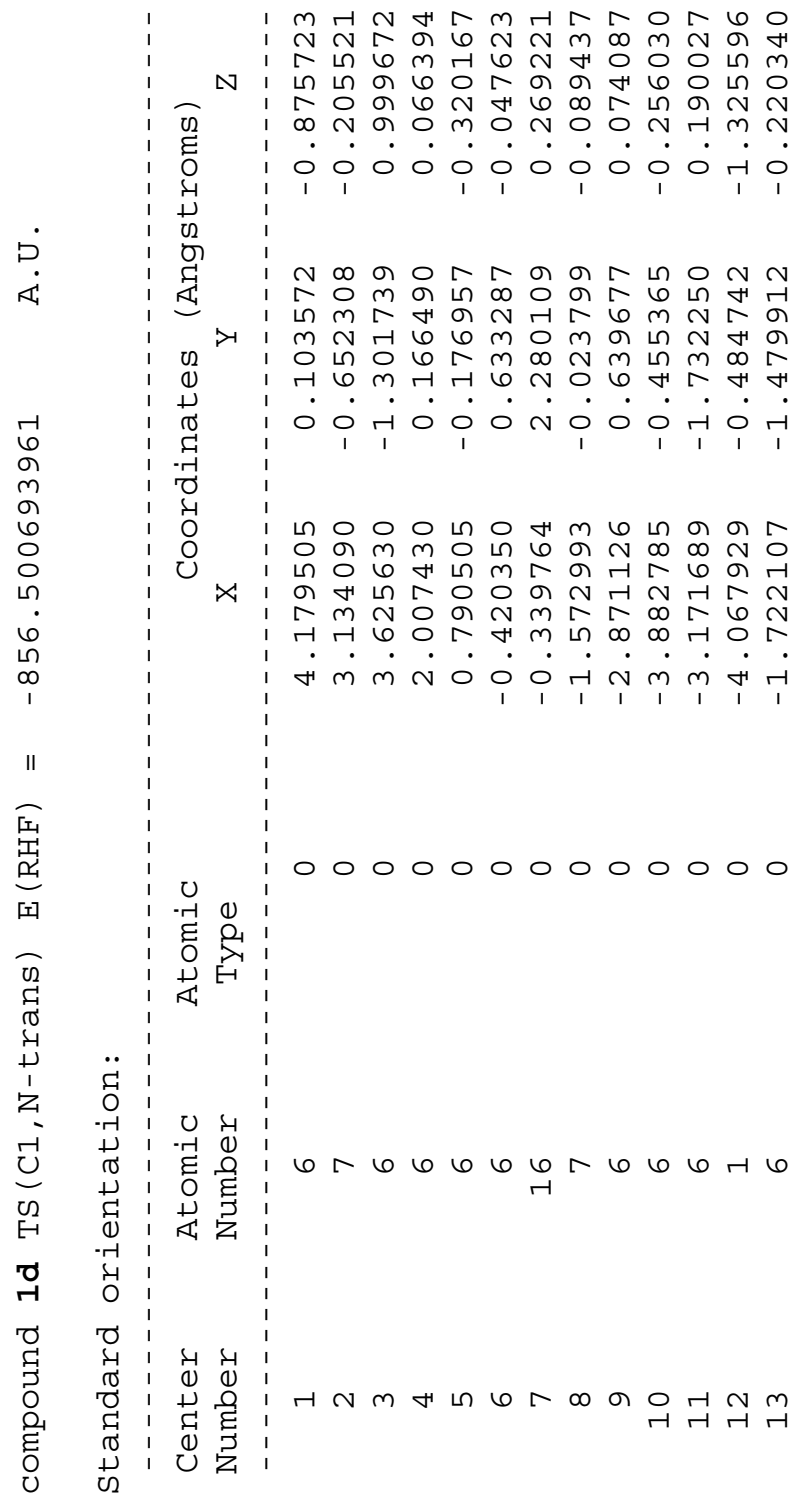


๓

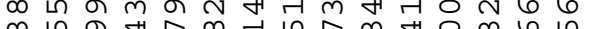

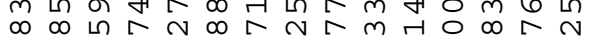

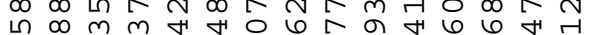
N 1 H H

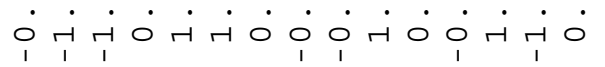

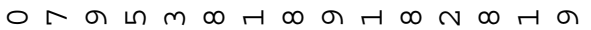

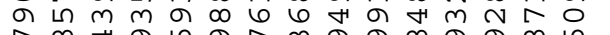

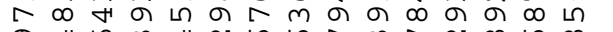
の ○ $m 6$ の

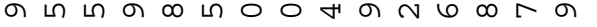

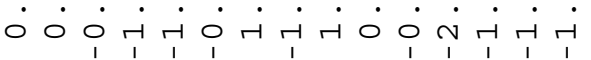

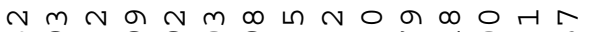
$\infty$ ब 6 の $N$ m

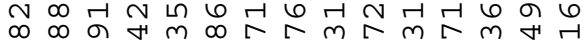

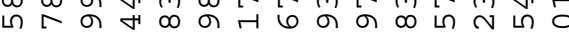

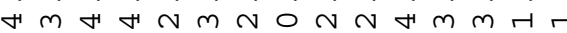
000000000000000

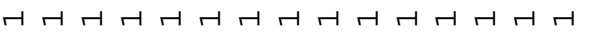

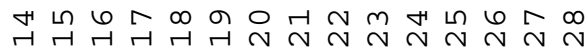

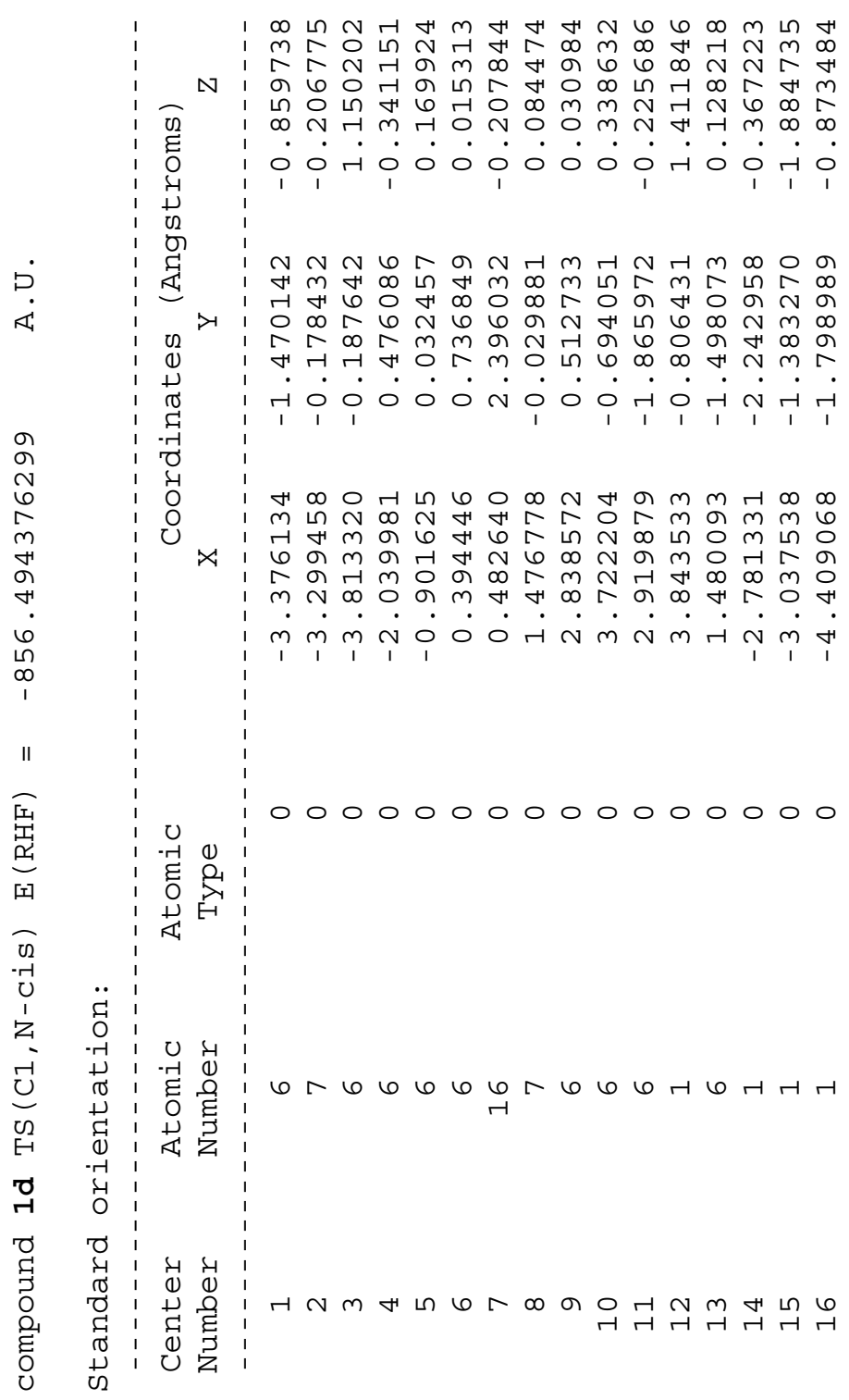




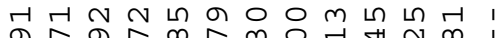

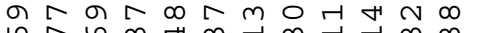
n m $r$ เ $\infty$ बर $\begin{array}{rllllllll}4 & 0 & 0 & 0 & 0 & 0 & 0 & 0 & 0\end{array}$

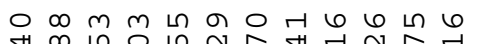

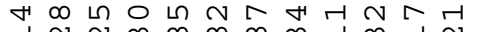

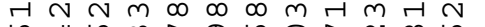
6 स 6 m

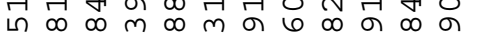

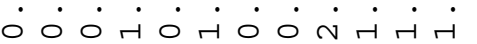

$m+6 \circ \wedge \infty m \wedge 6$ H० A 4 小 अ 10 เ

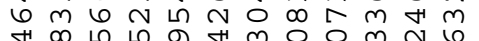

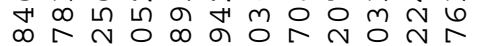

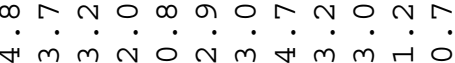
000000000000 $44444-4944-4$

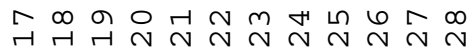
imm $n$ on m

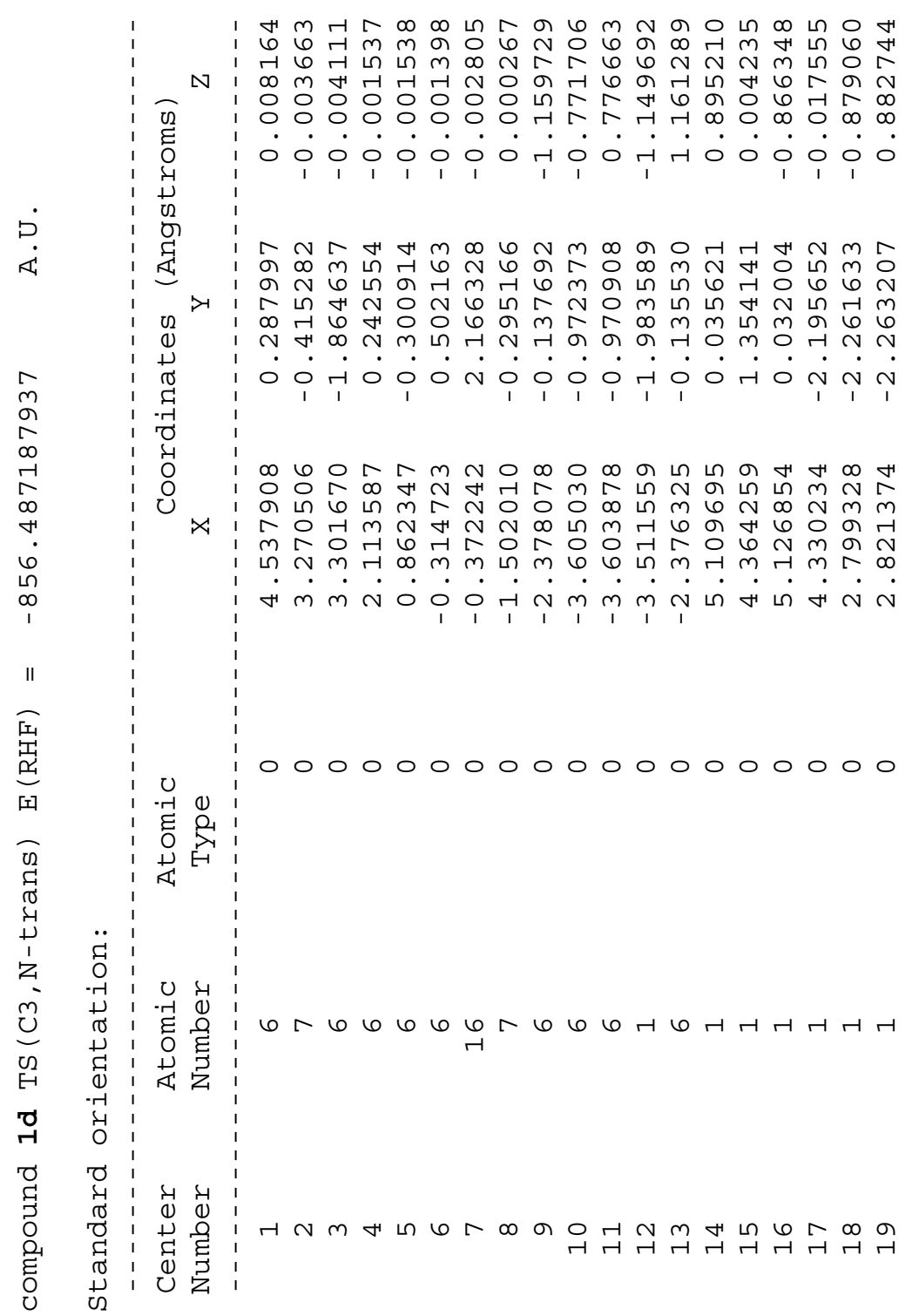

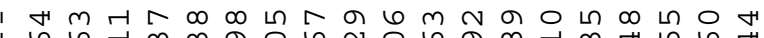
$\infty \mathrm{m}$ ॠ

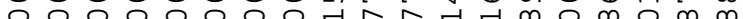
$\dot{0} \dot{0} \dot{0} \dot{0} \dot{0} \dot{0} \dot{0} \dot{0} \dot{0} \dot{0} \dot{0} \dot{0} \dot{0}$

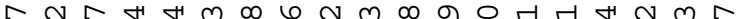

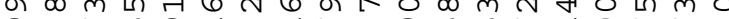

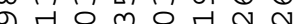

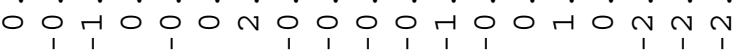

$\circ \infty \circ \infty$ の ம $\infty N N$ N

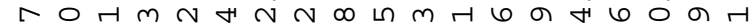

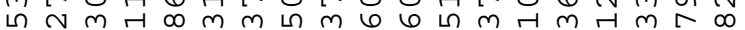
Hm n O O 


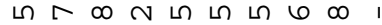
$m$ เ $०$ म $\circ \infty$ ก

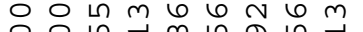
00 ए . 0 m $म$ मी $\dot{0} 0 \dot{0} \dot{H} \dot{H} \dot{r} \dot{\sim}$

$m \wedge 6$ เ $\sim \circ \infty$ ก ก 26 म $\infty \infty \curvearrowleft m$ ก 거 $m m$ म

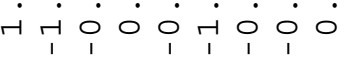

드 の の $\infty$ 구 $\infty$ 대 ब त त $1 \infty 6$ เ $\infty$ เก $\infty 6$

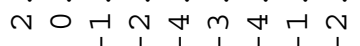

000000000

44444444

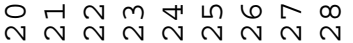

I $\infty$ เ

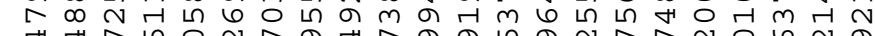

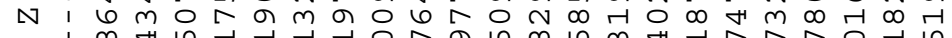
(2) 1 मान E 100 O 000 O 0 -

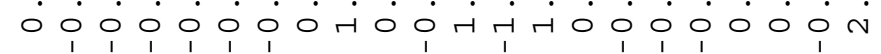

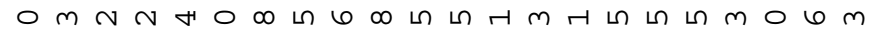

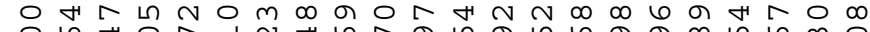
06 H 0 H $N 6$ \%

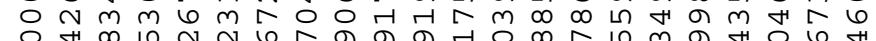

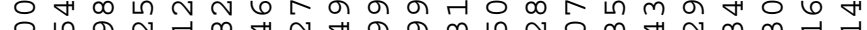

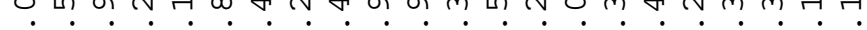

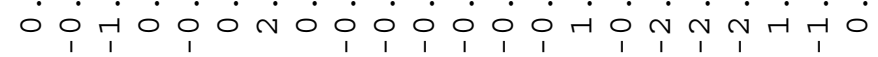

H ○ म $N \circ \mathrm{m} \omega$ เ ब 4 म $\infty$ म

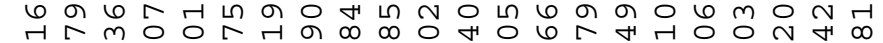

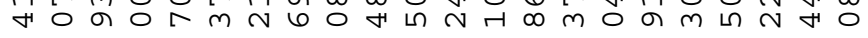

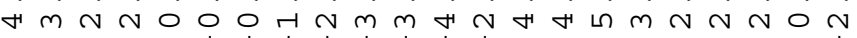




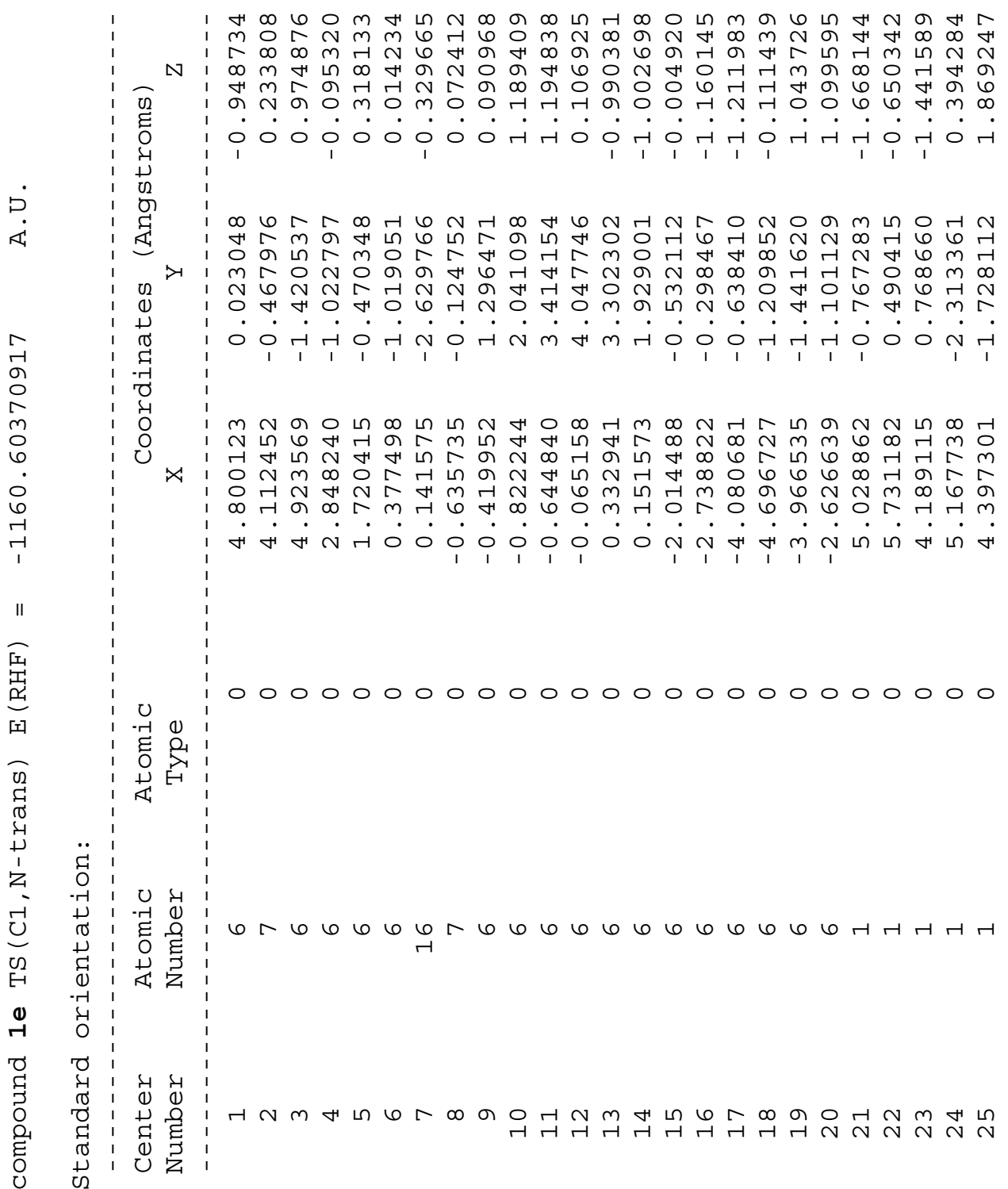


ก เ 당

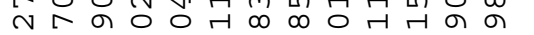

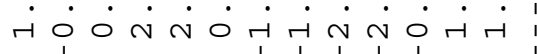

6ก

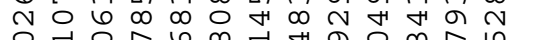
0 त $06 \infty$ म

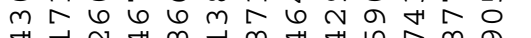
F

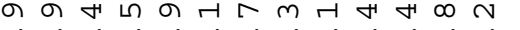
0

ब ब $\infty N$ औ

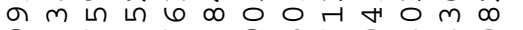
\%

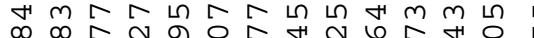
$\infty \infty \pi n$ a $\infty$ म

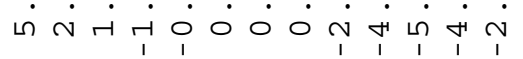

0000000000000

4444444444444

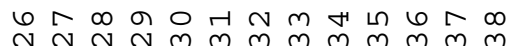

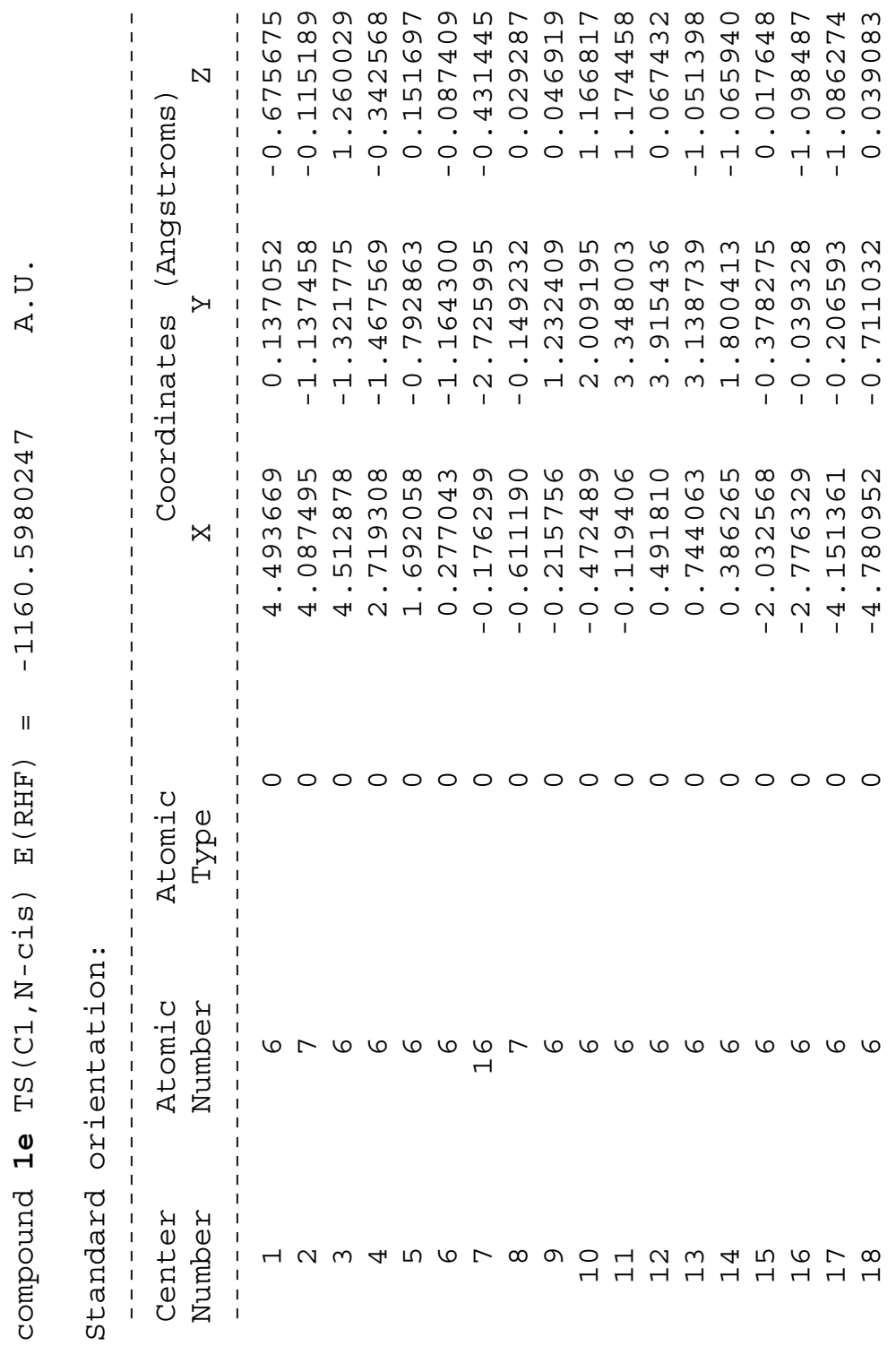


H 4 小

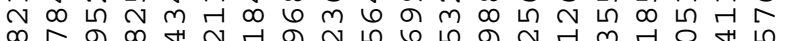

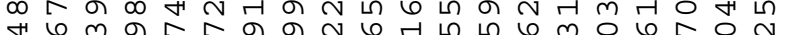
मे त

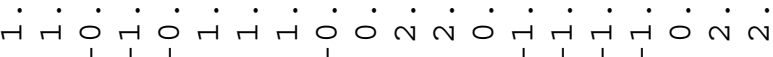

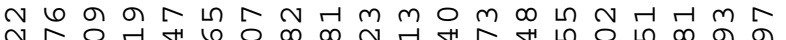

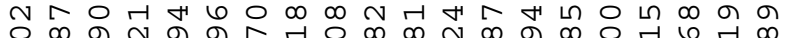

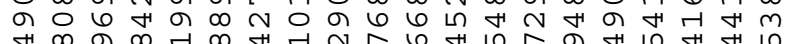

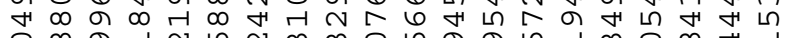

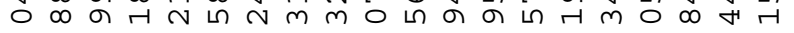

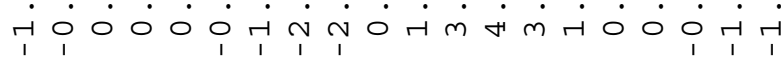

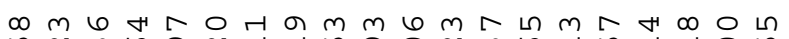

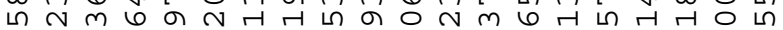

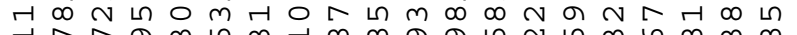
닷ํ

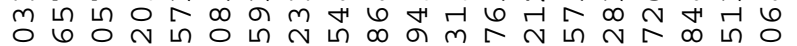

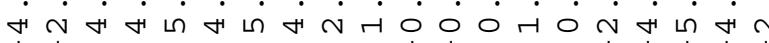
00000000000000000000 ש の

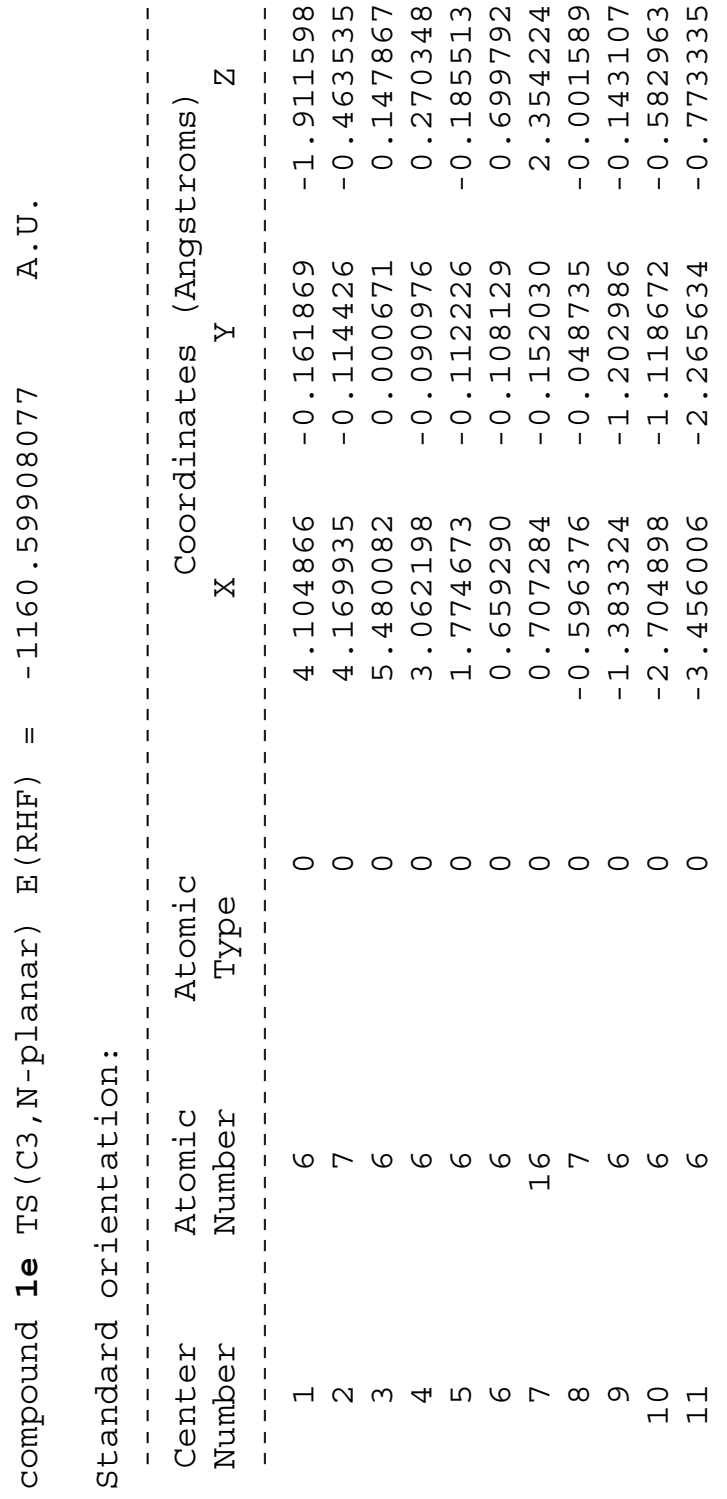


H 0600 の

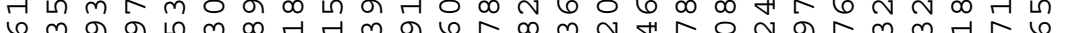

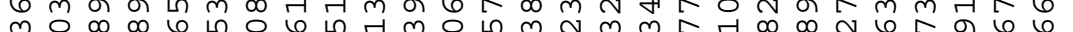

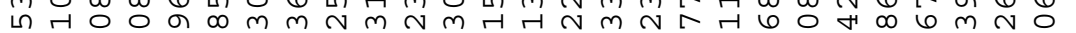

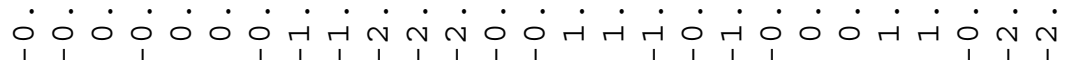

$\circ \infty$ の

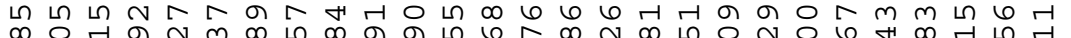

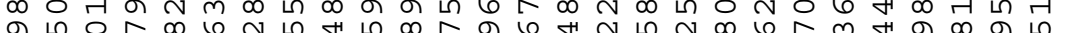

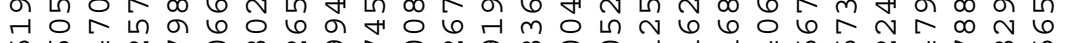

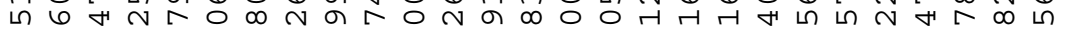

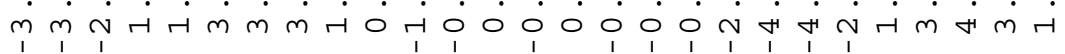

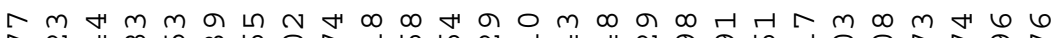

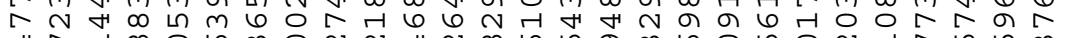

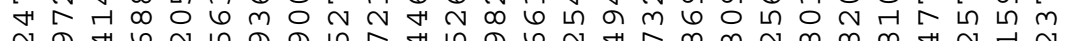
N

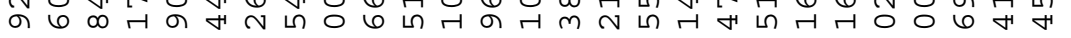

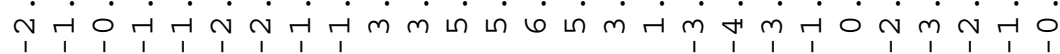

000000000000000000000000000

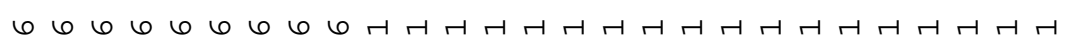

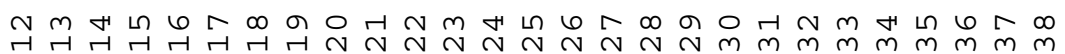

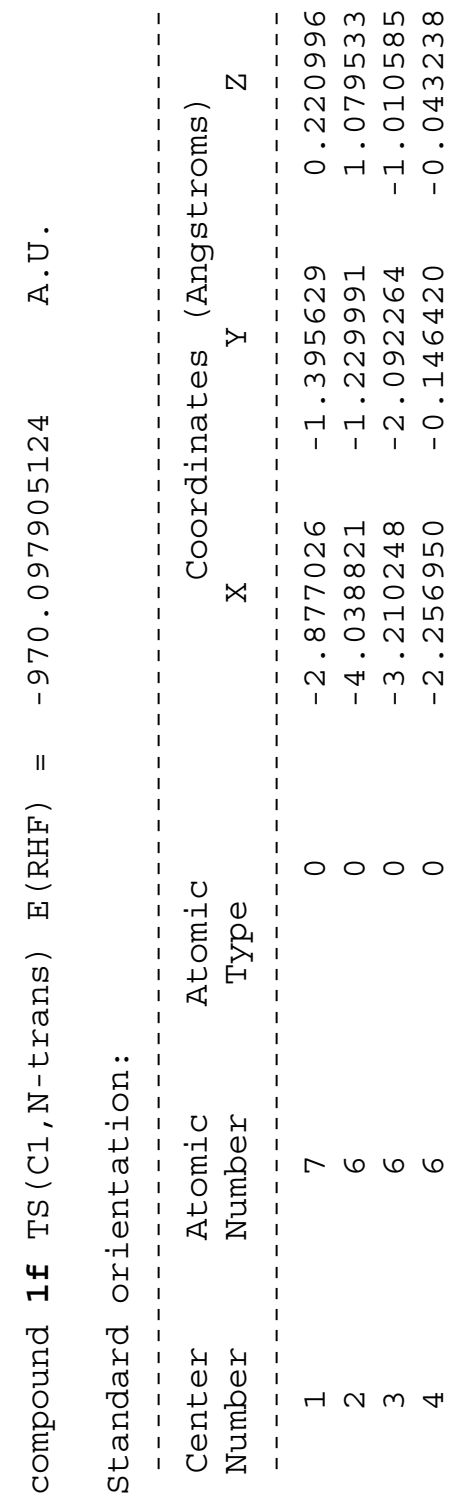


N $\infty$ O ब

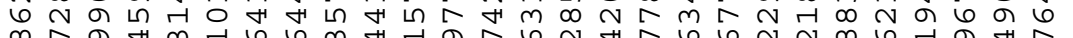

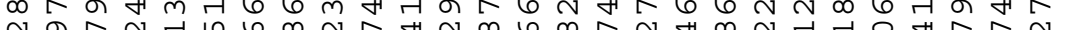
$\infty$ ๙ N 0 H 0 H 0 H

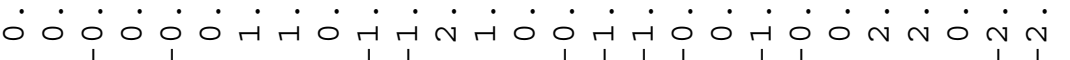

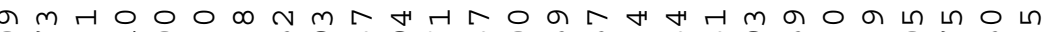
$\circ \Gamma$ H の 6 म $N$ ब $\infty 6$ ब $\infty 6$ の $N$ म $06 \mathrm{~N}$ त

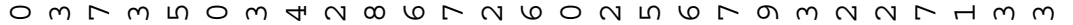

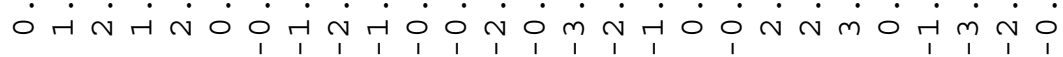

の

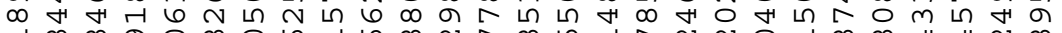

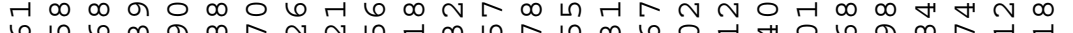
б

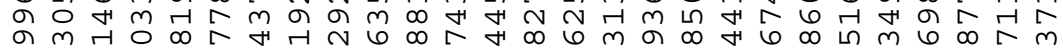

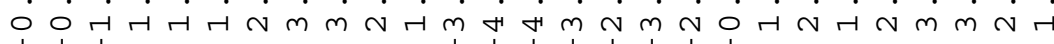
000000000000000000000000000 ๑

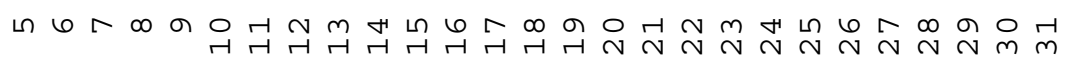

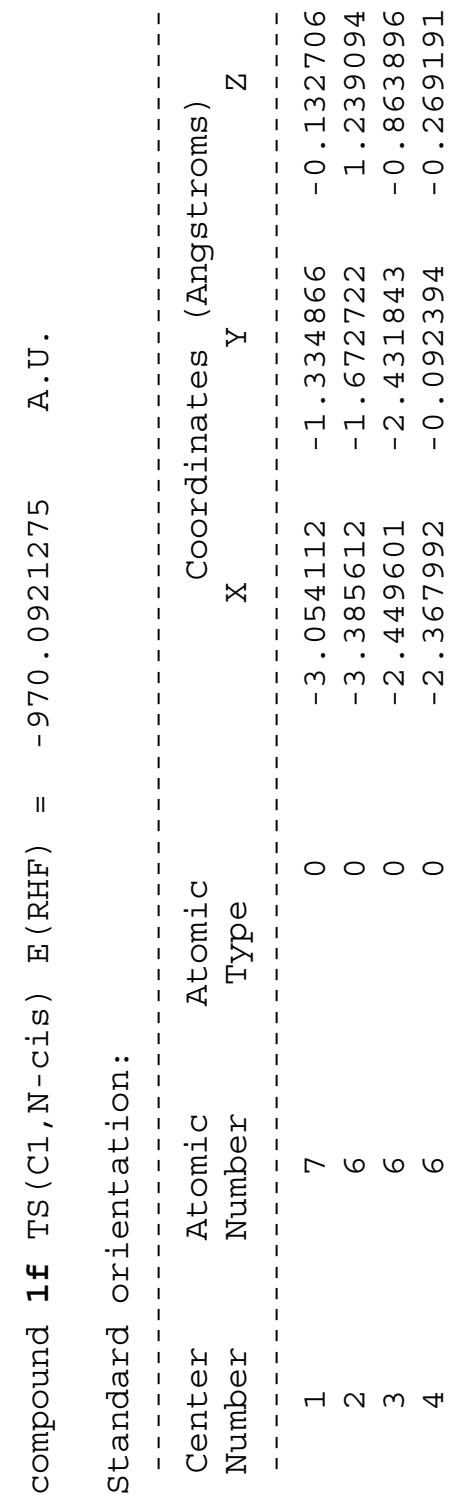


$\infty 0$ 대 $\infty$ เ

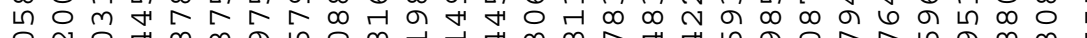

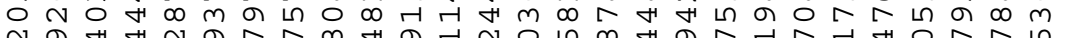
ब न

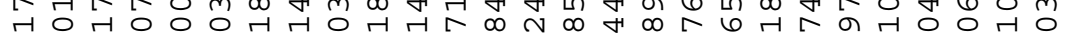

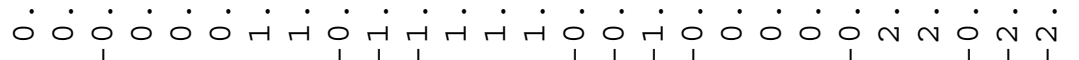

ब m 다

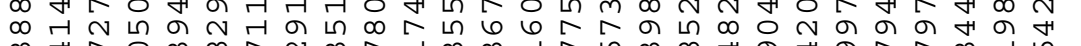

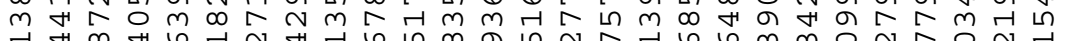

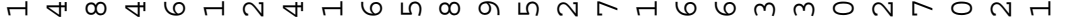

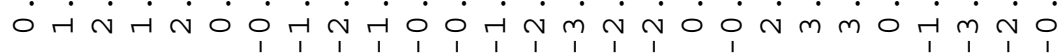

N 6 G

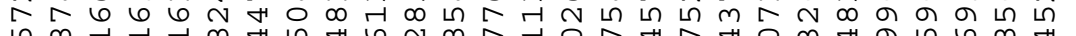

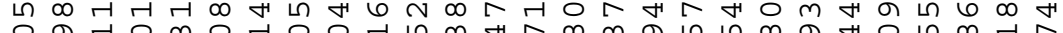
$\circ$ ॠ

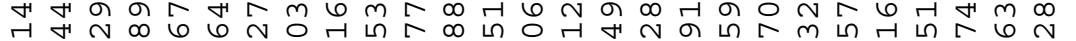

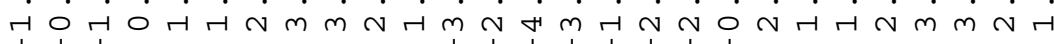

000000000000000000000000000

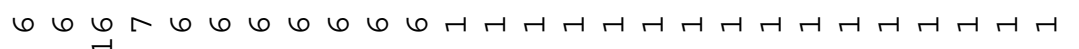

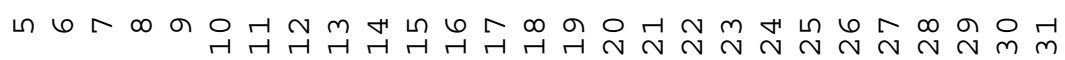

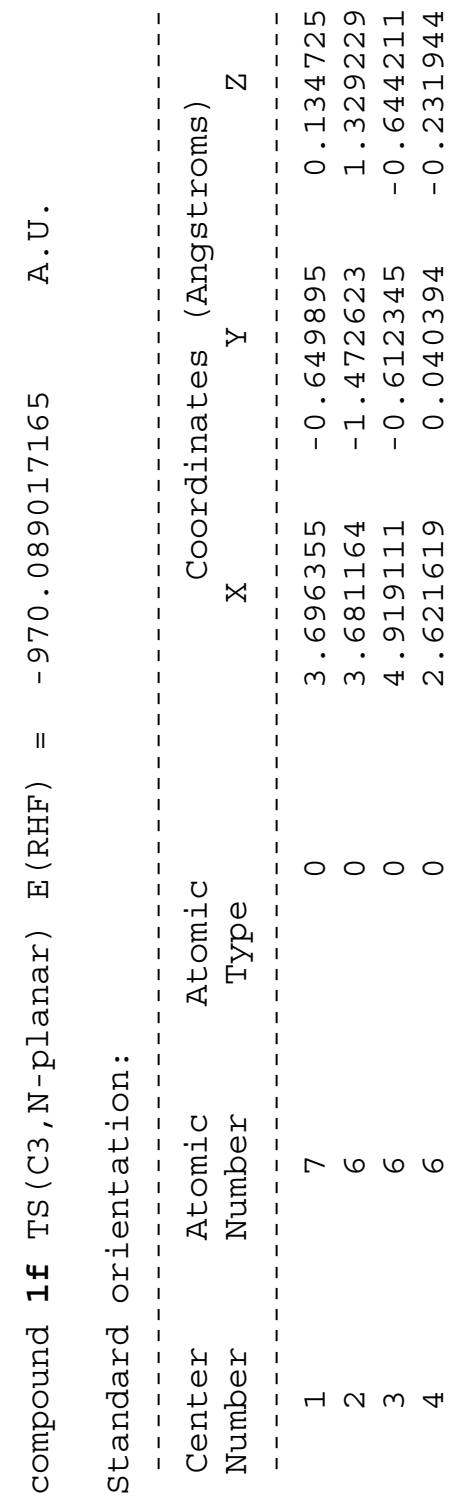


- のm

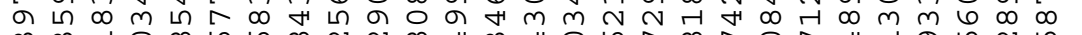

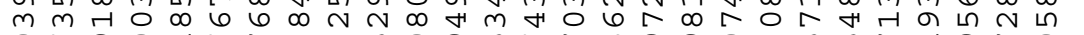

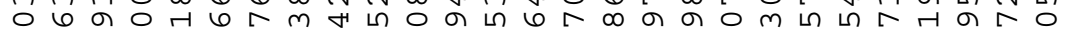
तर H०

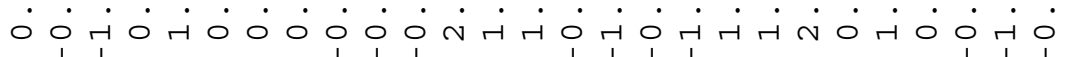
ח H $r$ H 小山 $\infty 6 m \Gamma$ 6 -

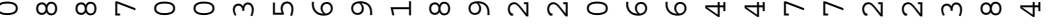

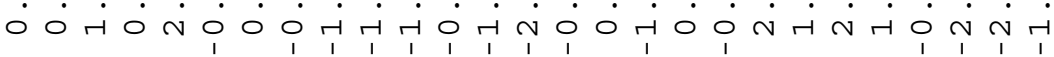

N 6 H

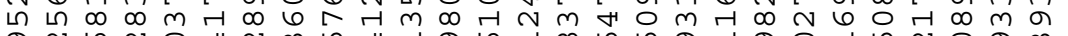

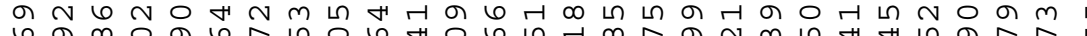

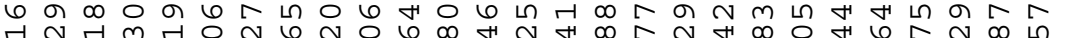

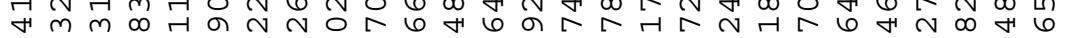

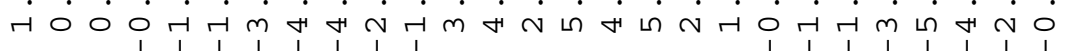
000000000000000000000000000 ๑ ம

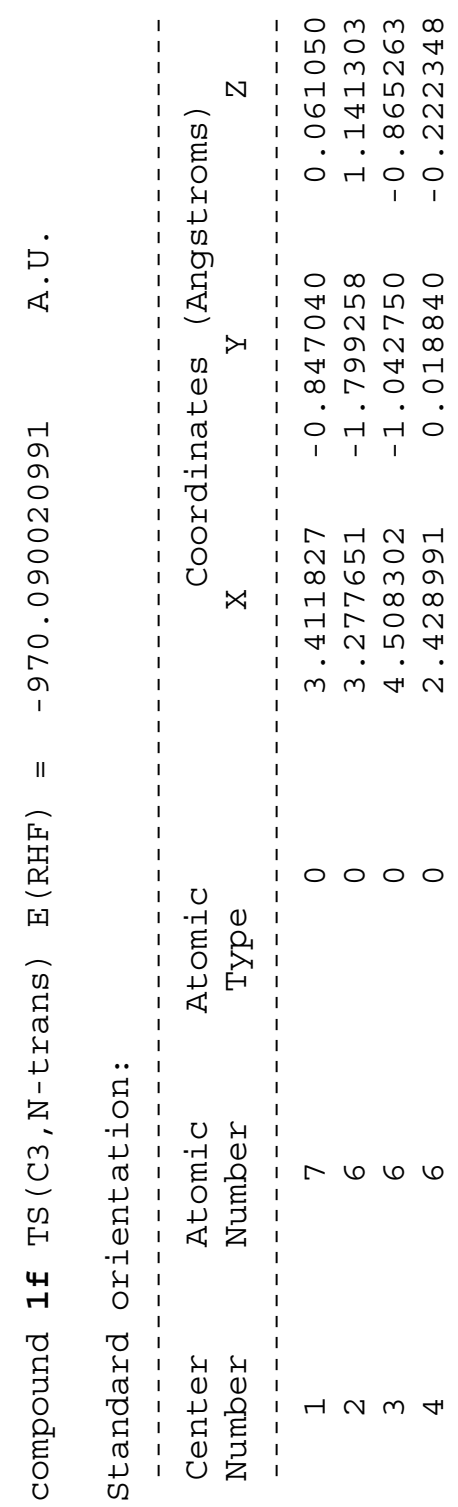




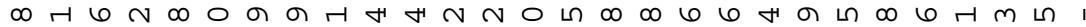

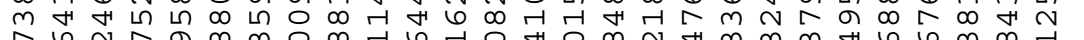

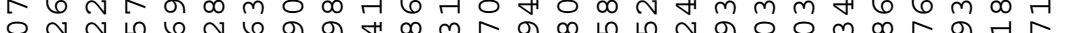
구 0 ก अ

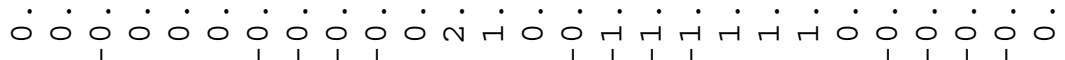

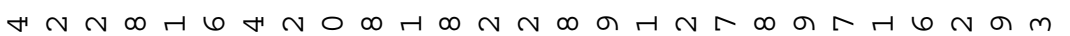

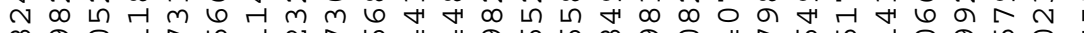
$\infty$ ब 0 त $m \sim\llcorner m+6$ ब

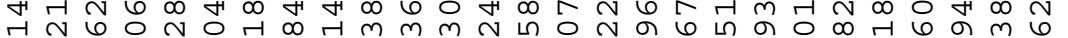

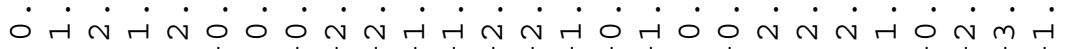

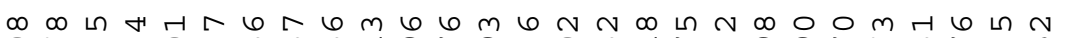
06 स

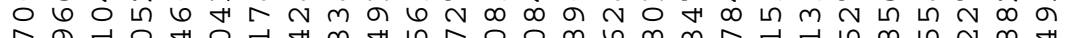
$\hat{m}$ न

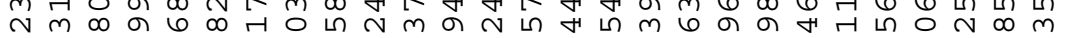

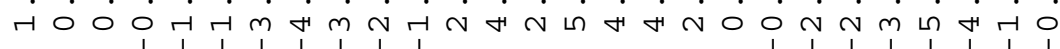

000000000000000000000000000

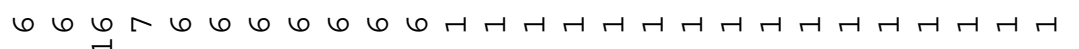

๓ $\curvearrowright$ の

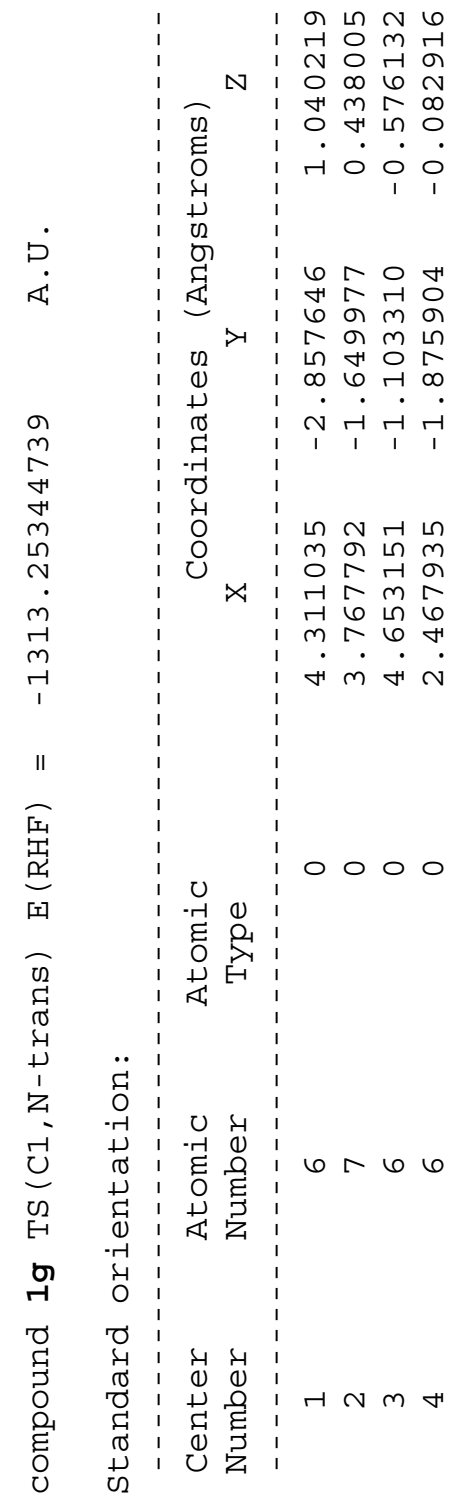




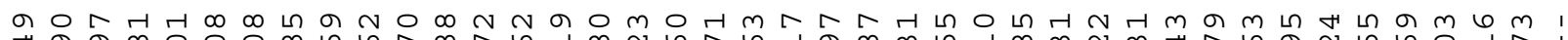
ॠ a 각

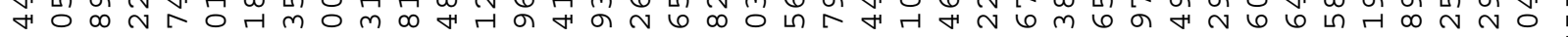

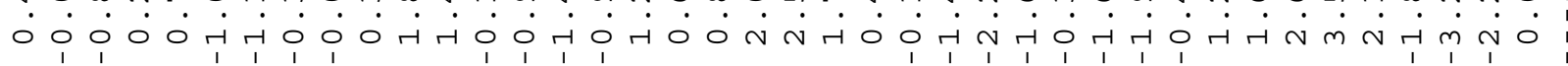

L m L L H L $\sim$ เ 6 अ

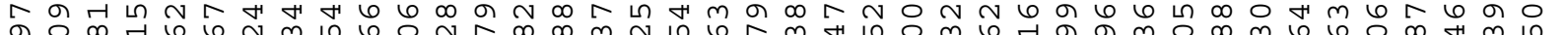

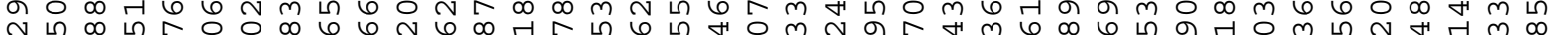

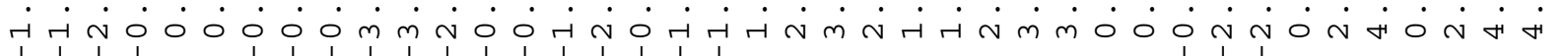

人

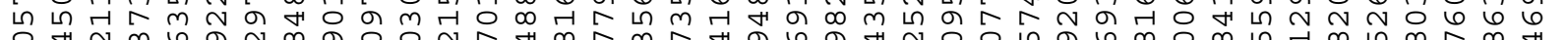

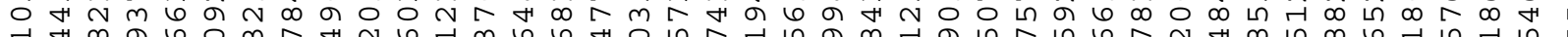

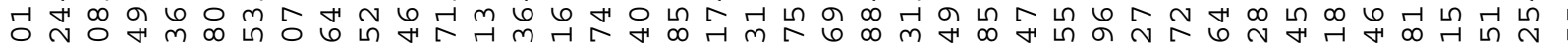

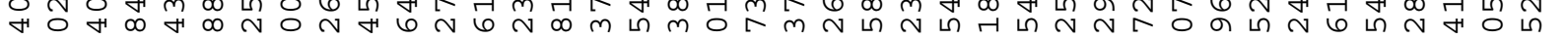

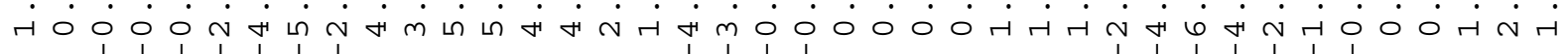
0000000000000000000000000000000000000000

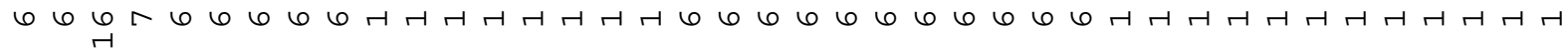
ம $6 \wedge$ व 


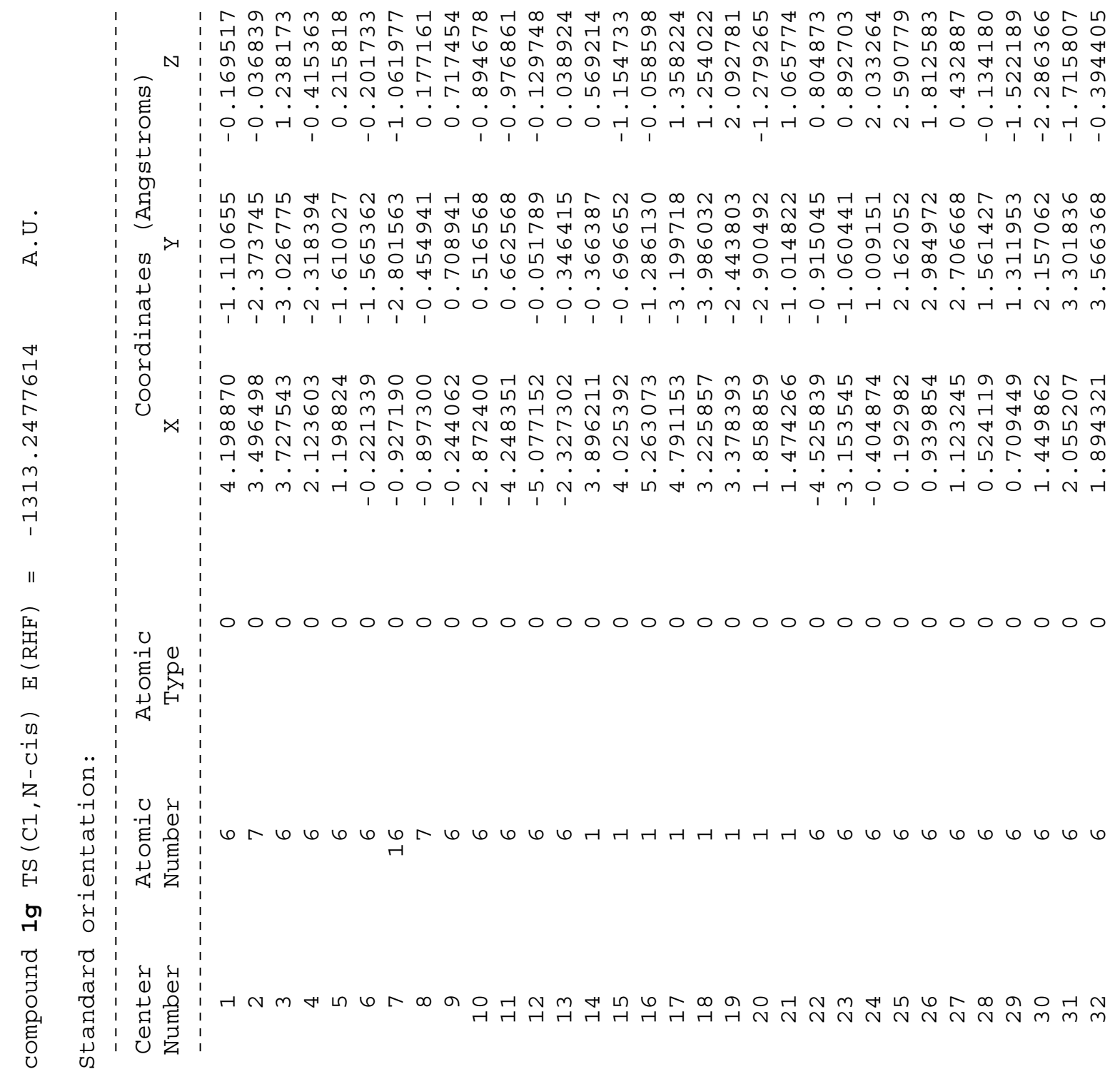


ก

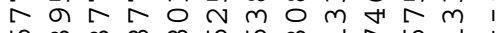

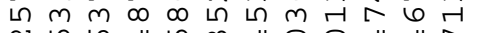
$N$ ก

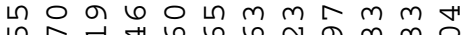

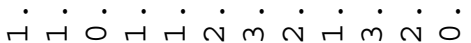

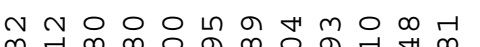

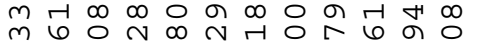

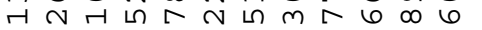

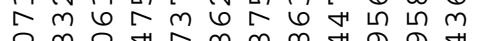

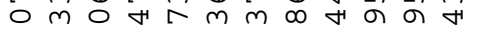

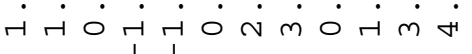

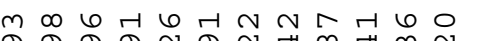

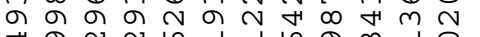
स $N N 6$ त $N 6$ ब $m$ ते

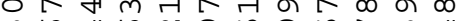
$m 6+6 N$ \% N 6 त

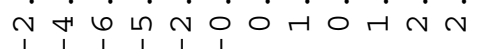

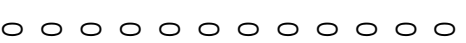

$44444-4944-4$

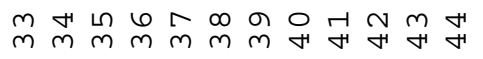

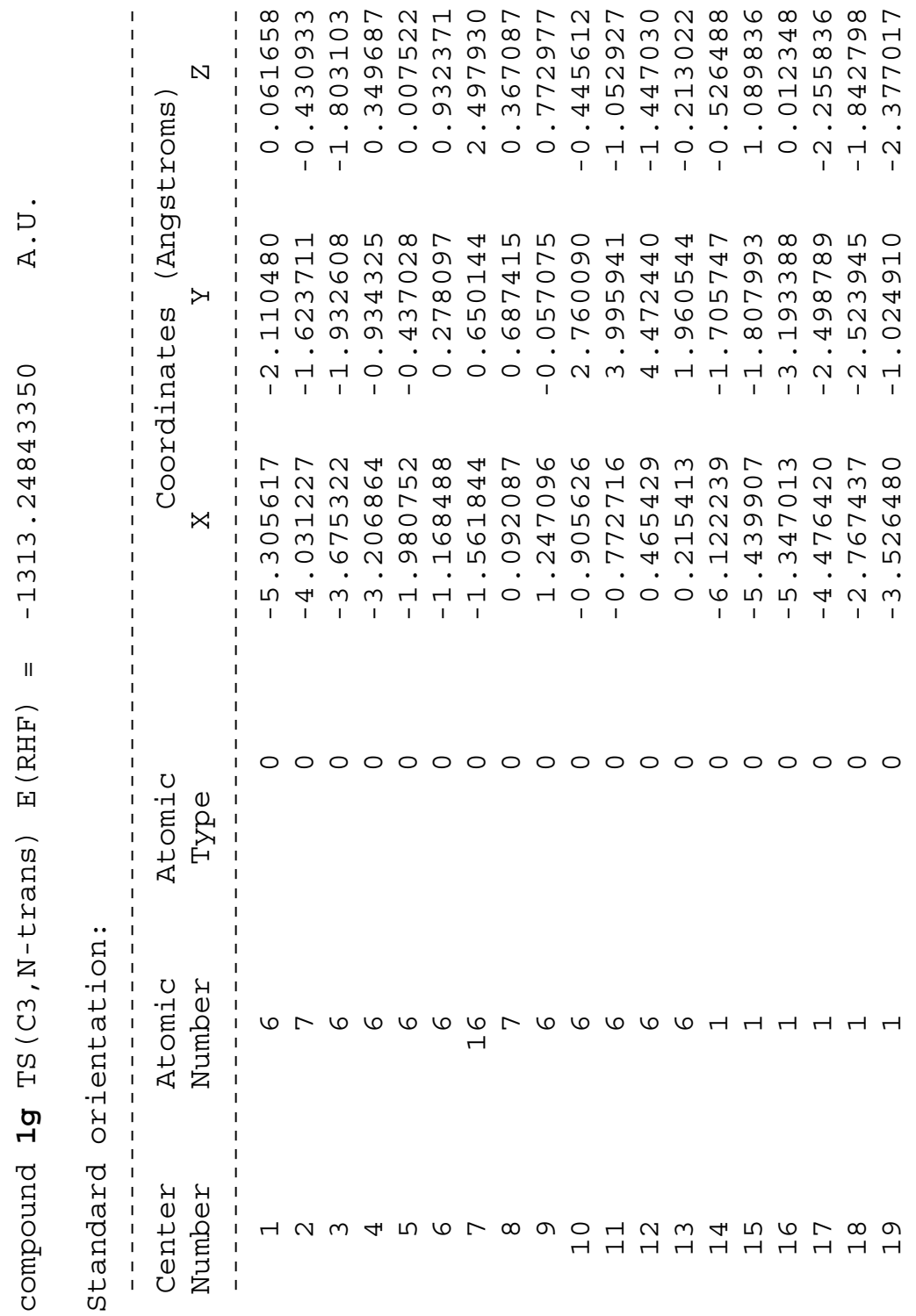

$\infty m m \Gamma N+O N N N$ N

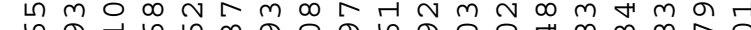
1

.


$m+4 \pi$ म नु の 4⿵

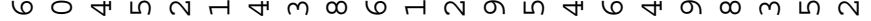

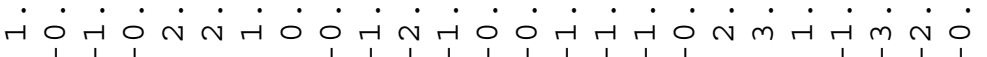

N 6 अ ए

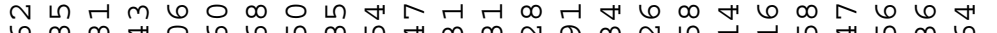

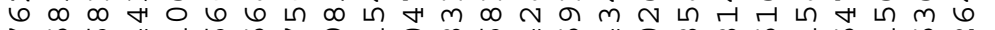
ก म 6 म

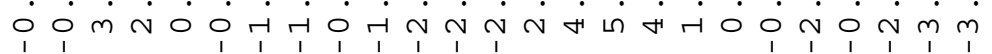

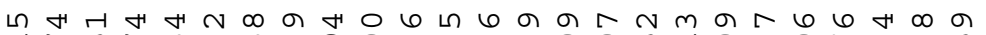

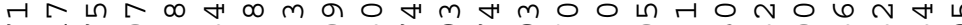

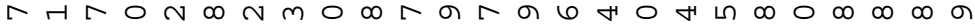

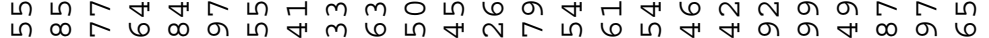
เก ก

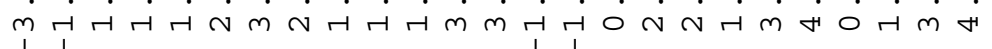
0000000000000000000000000 म

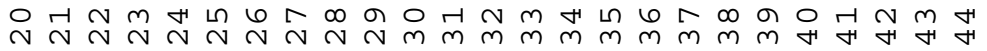

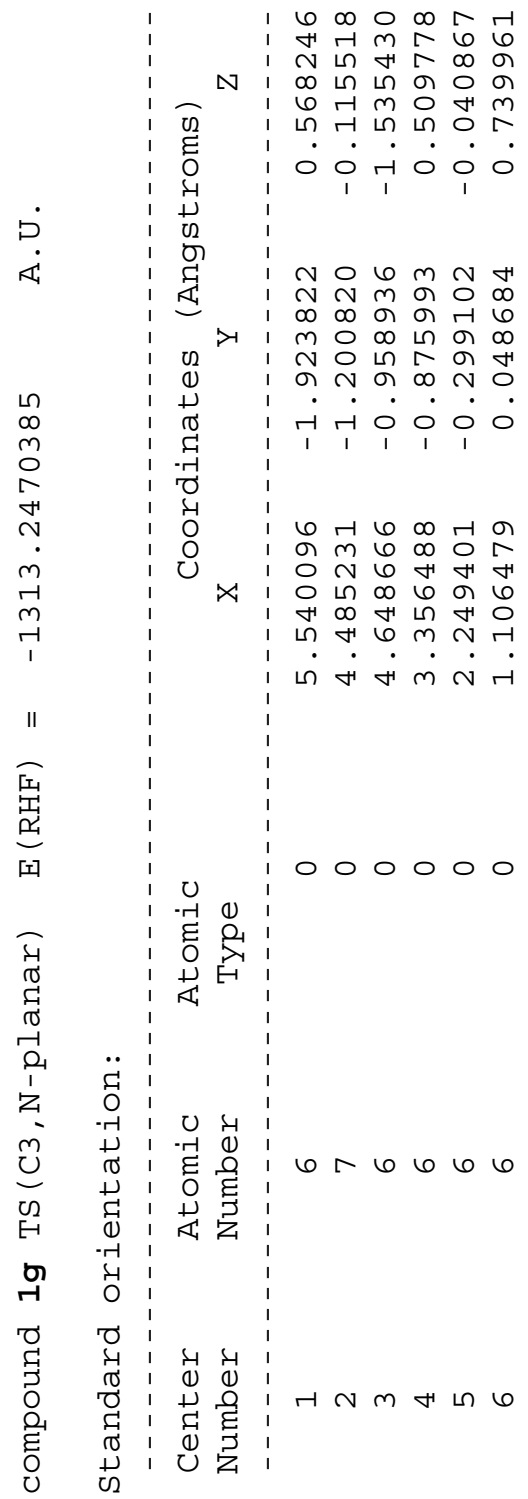


- - H

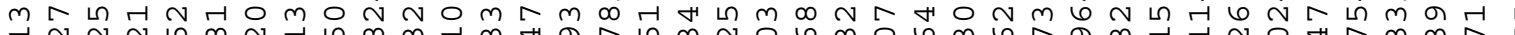

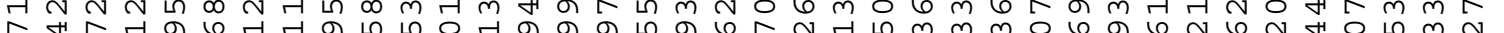
$\infty$ 군

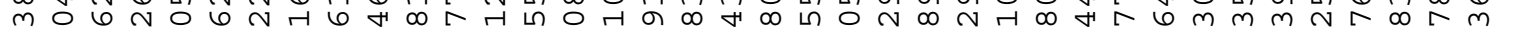

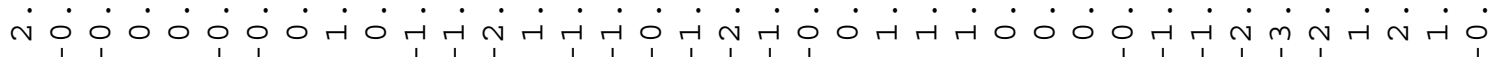

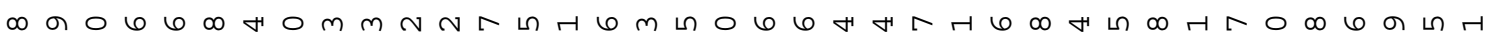

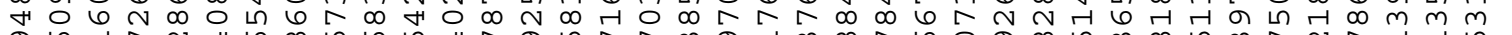

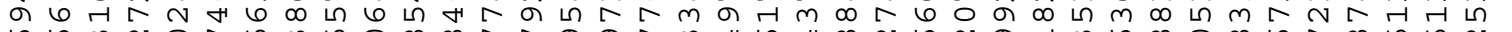

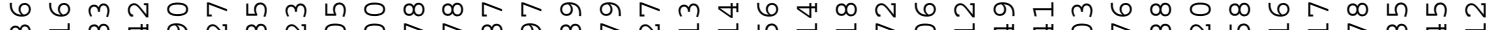

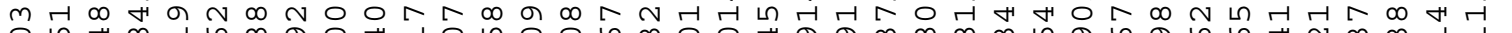
0 เ

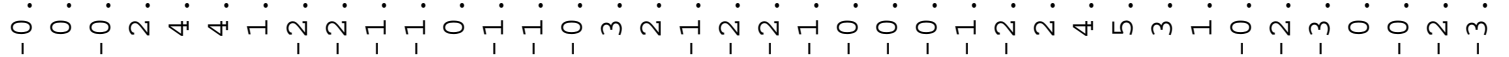

a $6 \operatorname{th}$ H

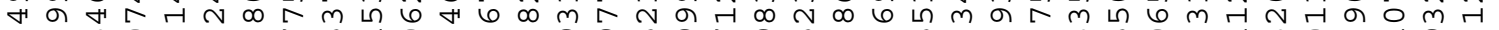
N 으유 の 0 ○

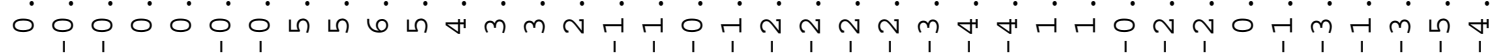
00000000000000000000000000000000000000 6

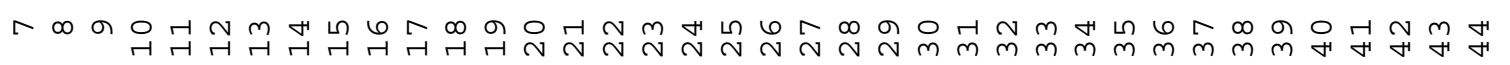




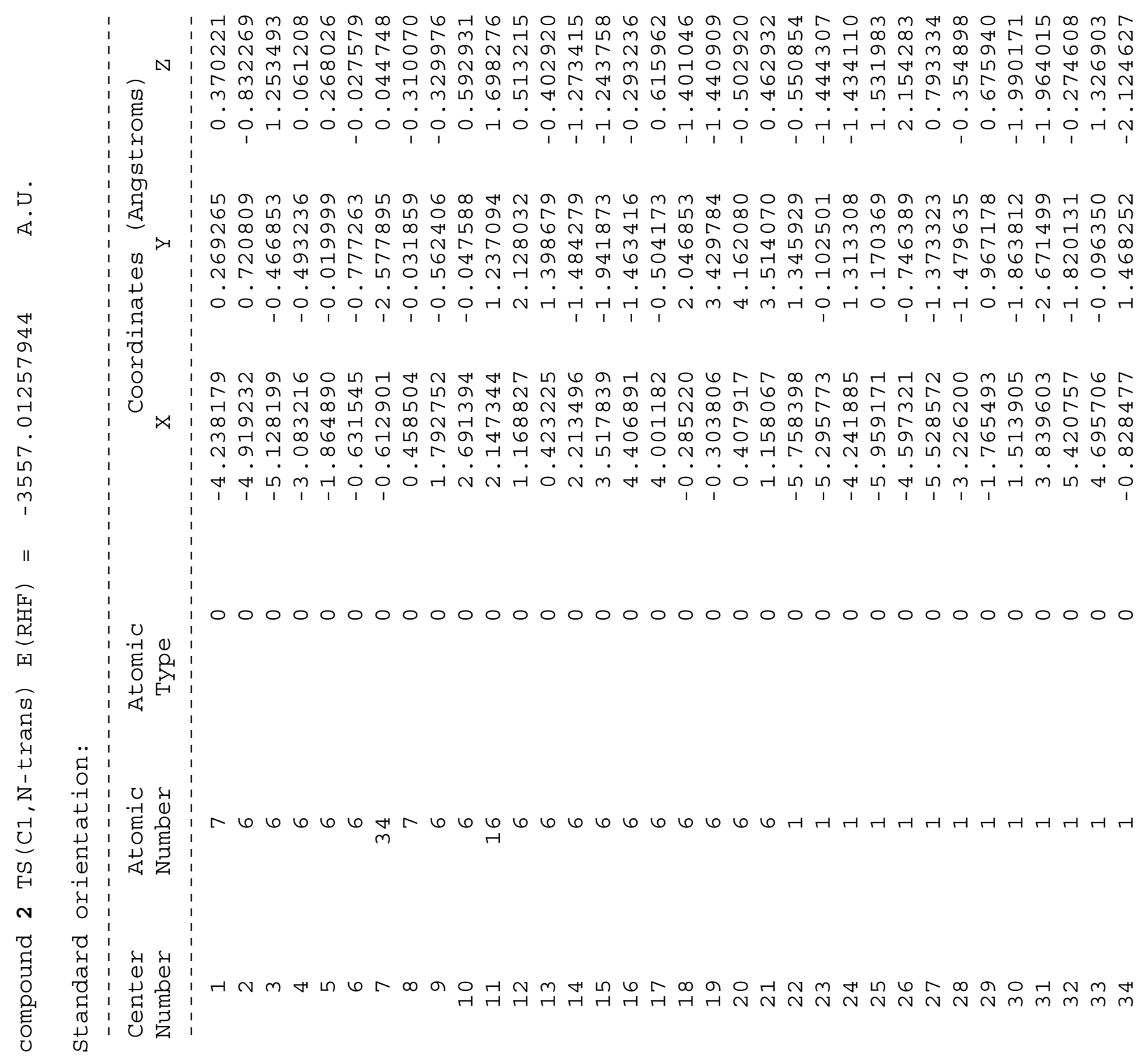




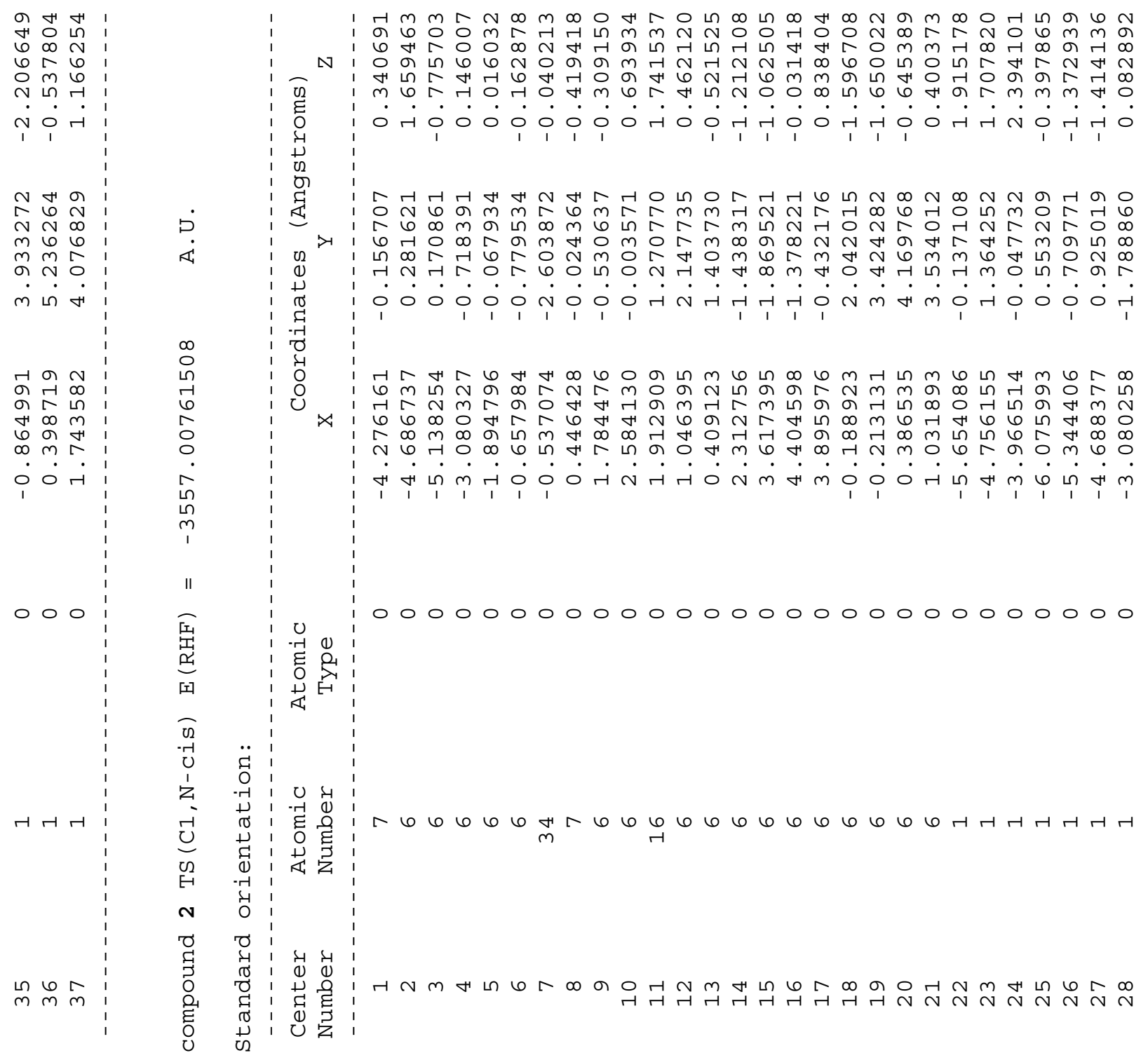


अの $\circ \mathrm{m}$ \% $\mathrm{m}$ / +6

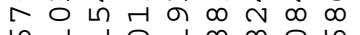

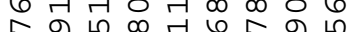
व $106 \mathrm{~m}+6$. $\dot{0} \dot{H} \dot{0} \dot{\sim} \dot{\sim} \dot{0}$

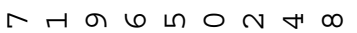
$\circ \infty \infty$ の $m \circ m$ 다

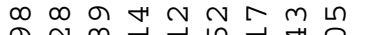
ब $\infty$ म の $\infty$ เ

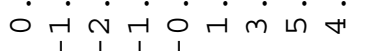

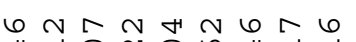
$A 40, \pi$,

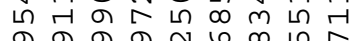
ब

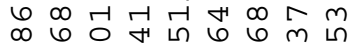
ते

000000000

Н

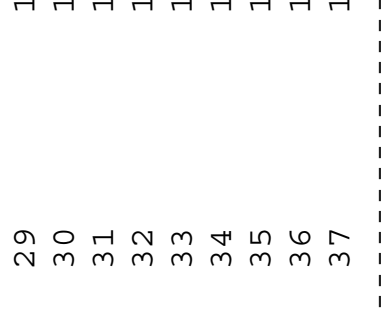
$\begin{array}{lll}1 & 1 & 0 \\ 1 & 1 & 1\end{array}$

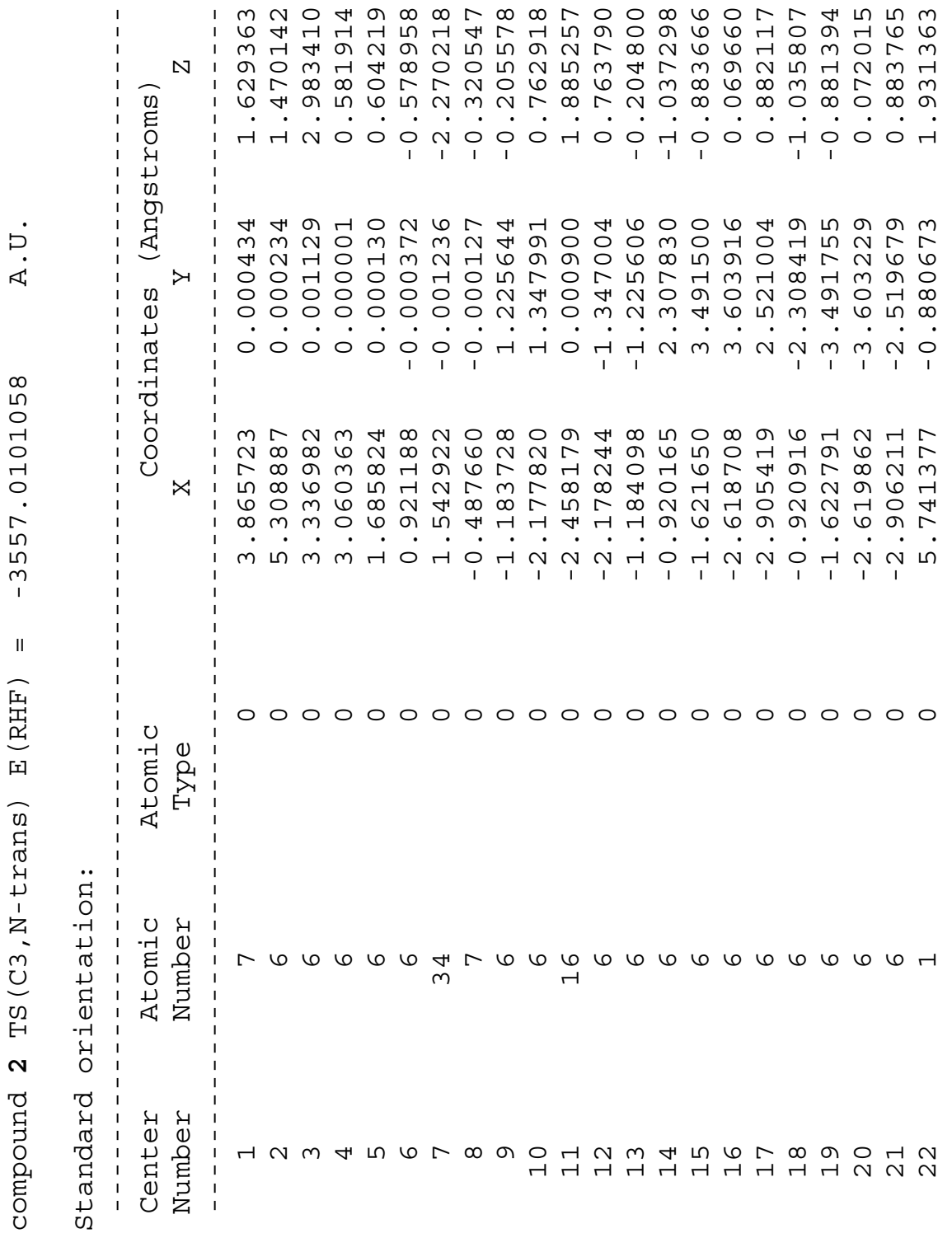

m N

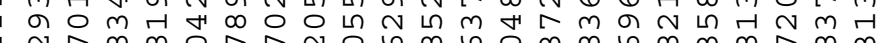

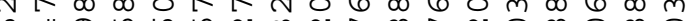

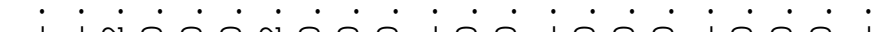
H $m m N \circ m \pi m N$ H म $N$ H 0 . 西

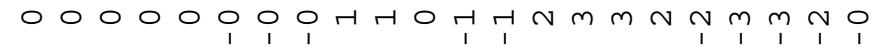

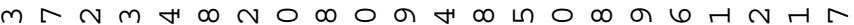

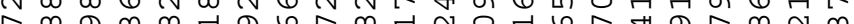
ก $\infty 60, \infty$

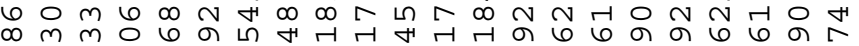

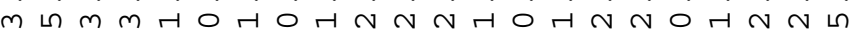


の $0 N \sim$ N अ $6 m$ 다 0 - $\sigma$

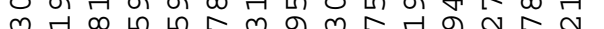
ब

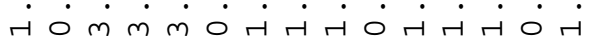

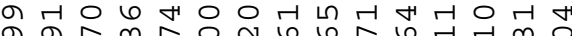

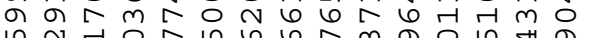

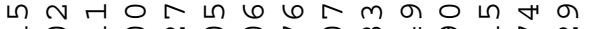
익

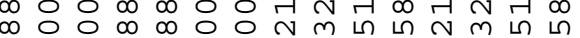

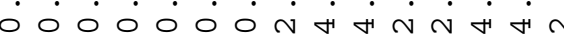

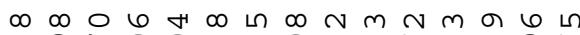
$N$ G $\mathrm{N} \circ \mathrm{m} m \circ \mathrm{N}$ \%

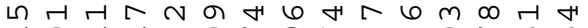
다

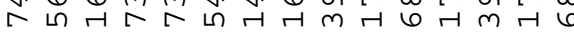

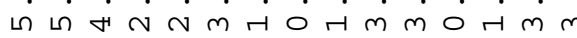
000000000000000

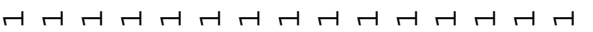

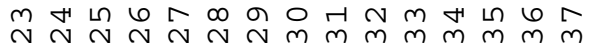

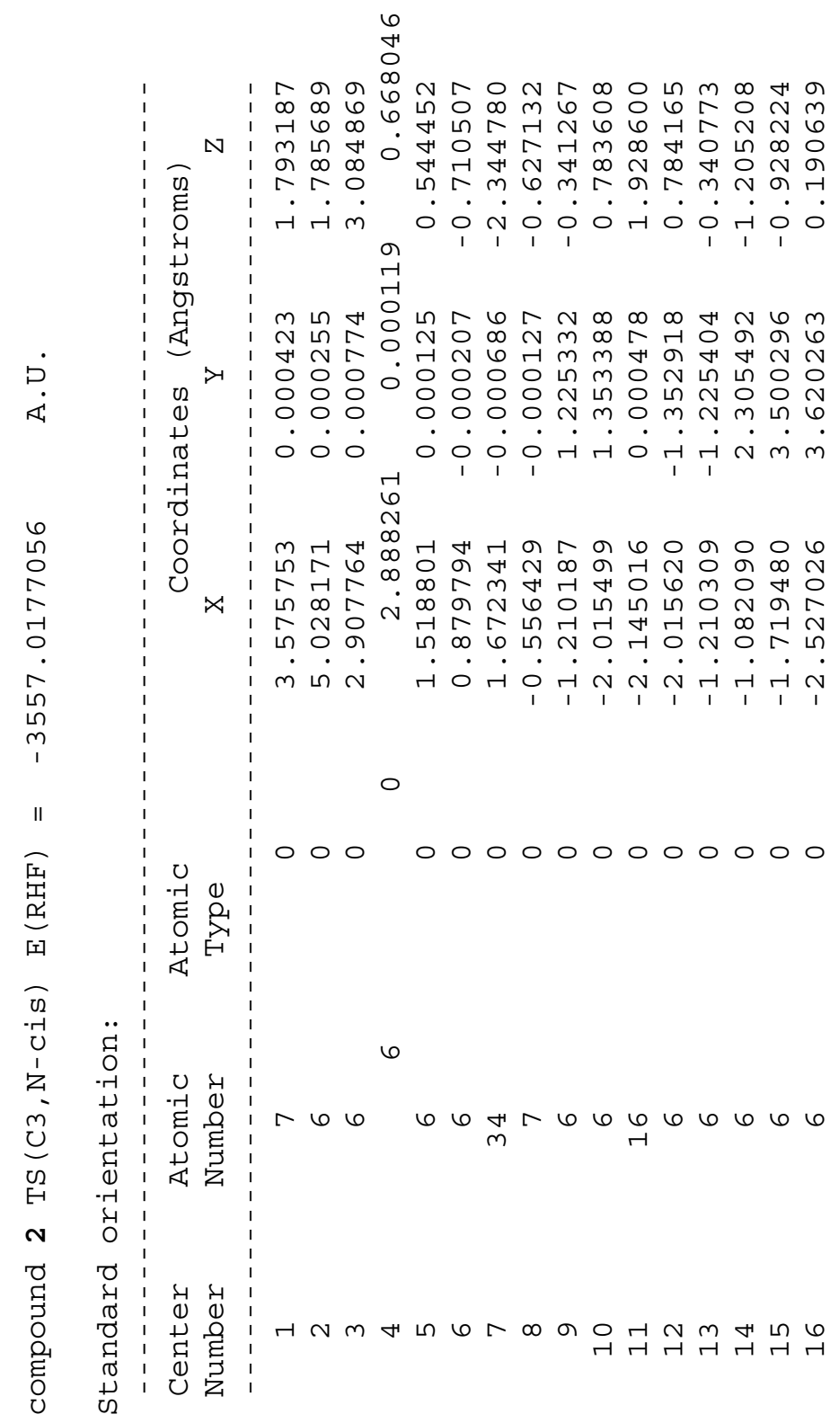


$m$ m $m 0$ \%

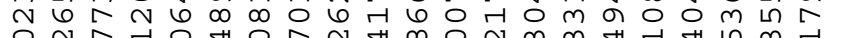

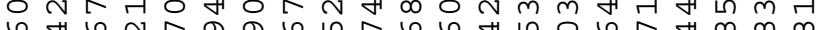

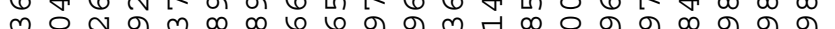
0 ๙ $\dot{H} \dot{0} \dot{0} \dot{0} \dot{\sim} \dot{0} \dot{m} \dot{m} \dot{m} \dot{0} \dot{0} \dot{0} \dot{0} \dot{0}$

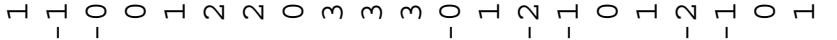

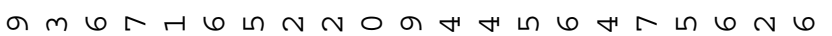

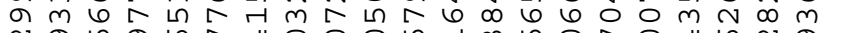

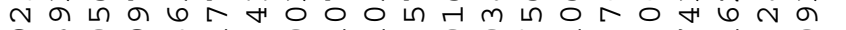
ब

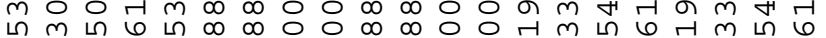

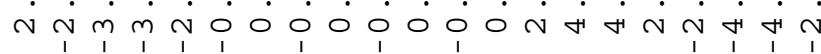

$m$ m 2 ก

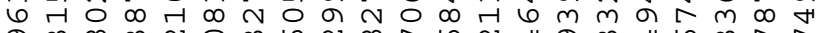

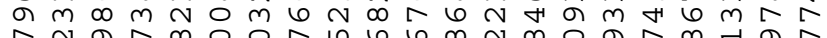

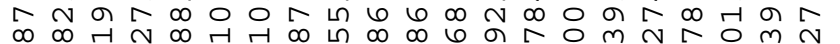

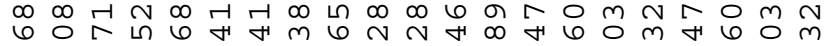

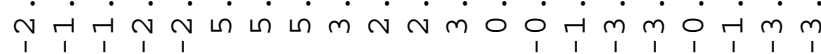

000000000000000000000

6 6 6

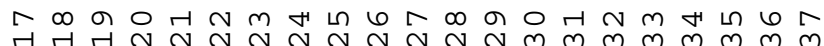

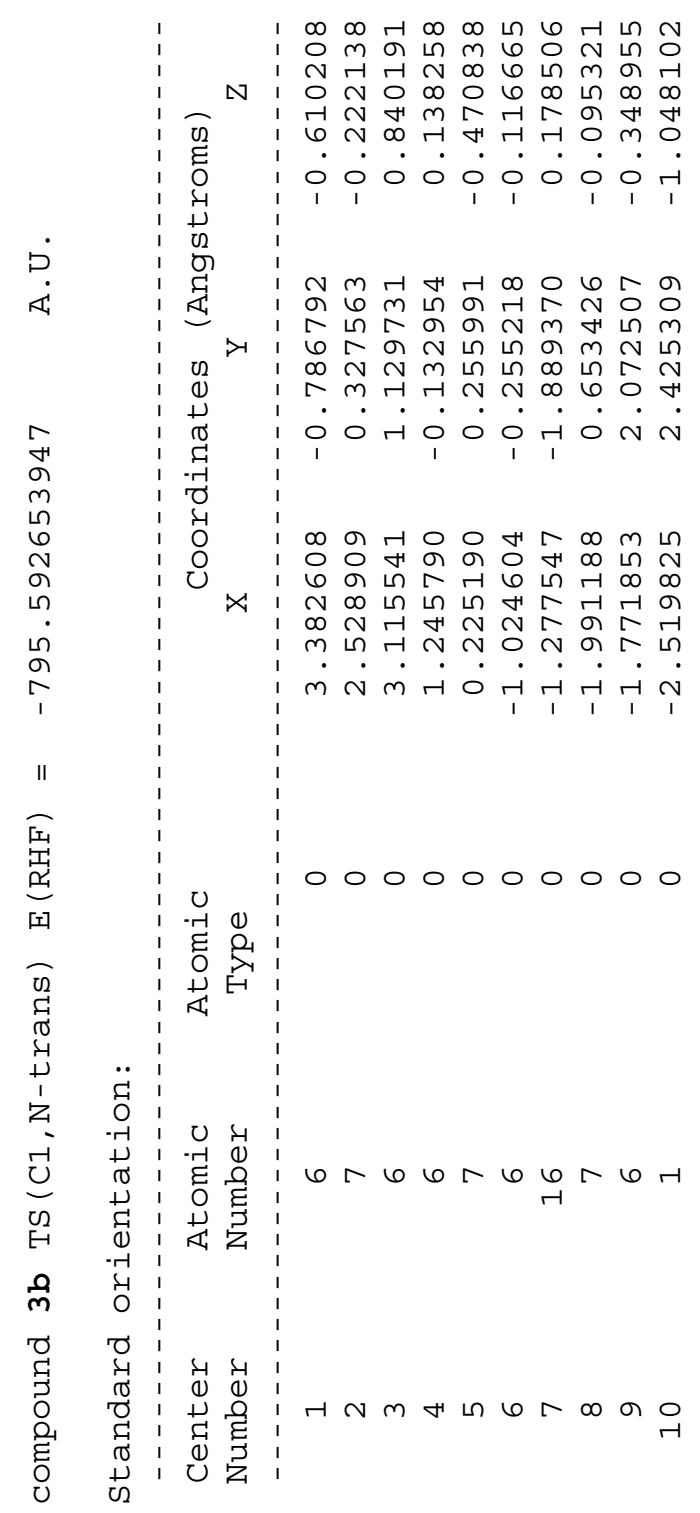


の $m 6 \infty 6 \infty 0$ H人OH

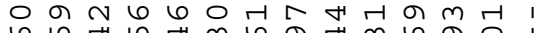

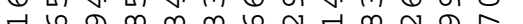

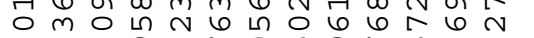
N N $\mathrm{N}$ ศ $\mathrm{r} 0$ เ

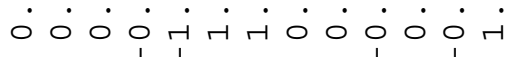

न ब $N$ - $N m m$ ब $N \infty$ 게 $m$ ब

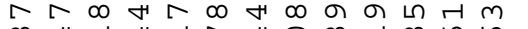
$\infty$ सं

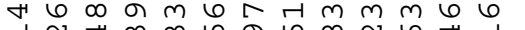
H $\mathrm{m} m$ เी

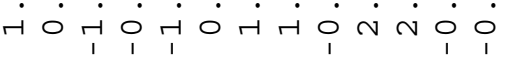

$04 \infty \pi$, N

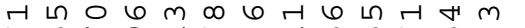

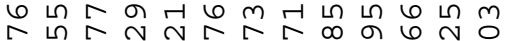
๙ิ ब $m$ เ $m$ ब $\dot{m} \dot{m} \dot{m} \dot{\sim} \dot{m} \dot{\sim} \dot{H} \dot{0} \vec{r} \dot{m} \dot{m}$ 0000000000000 4.44444444444

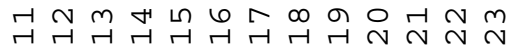

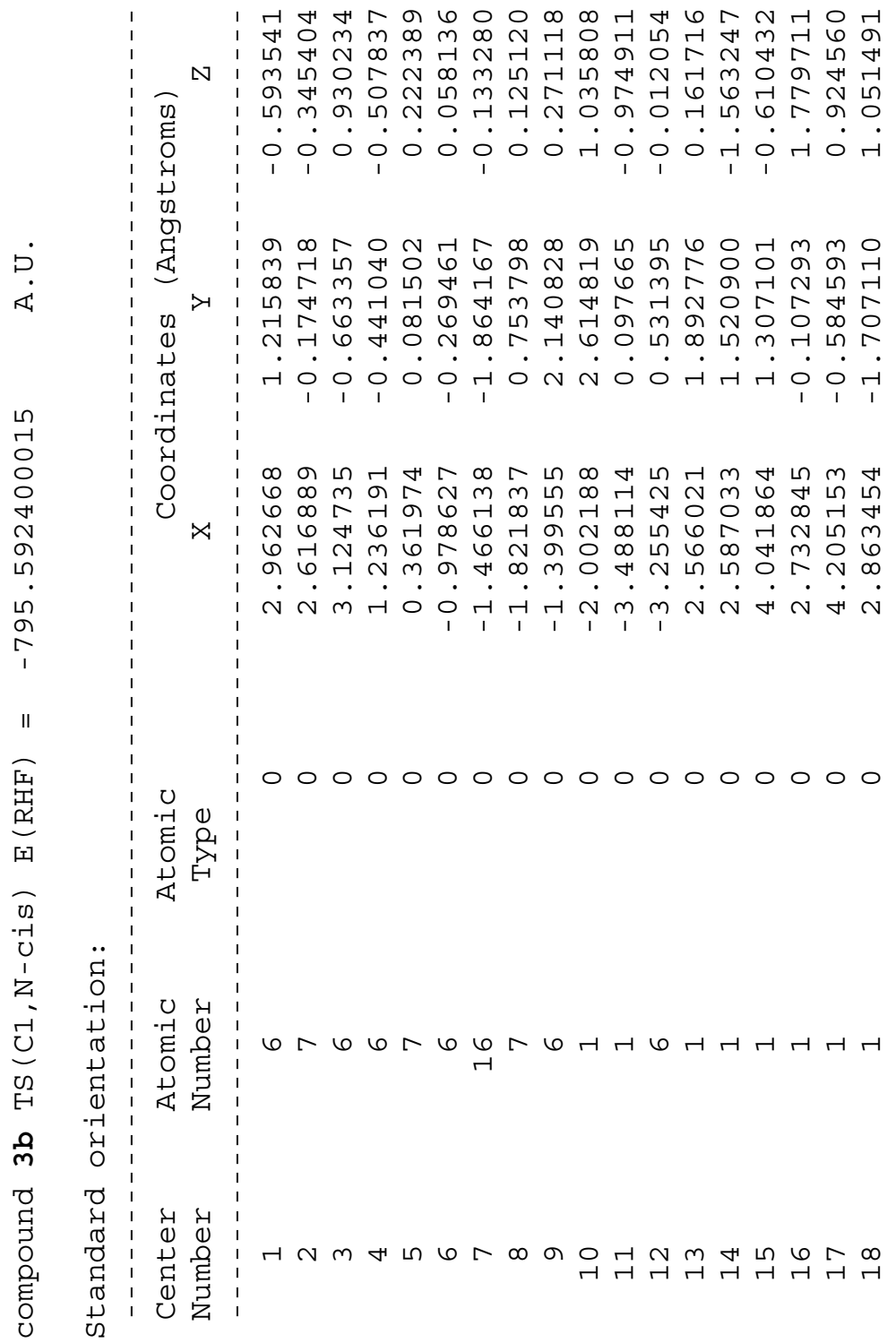




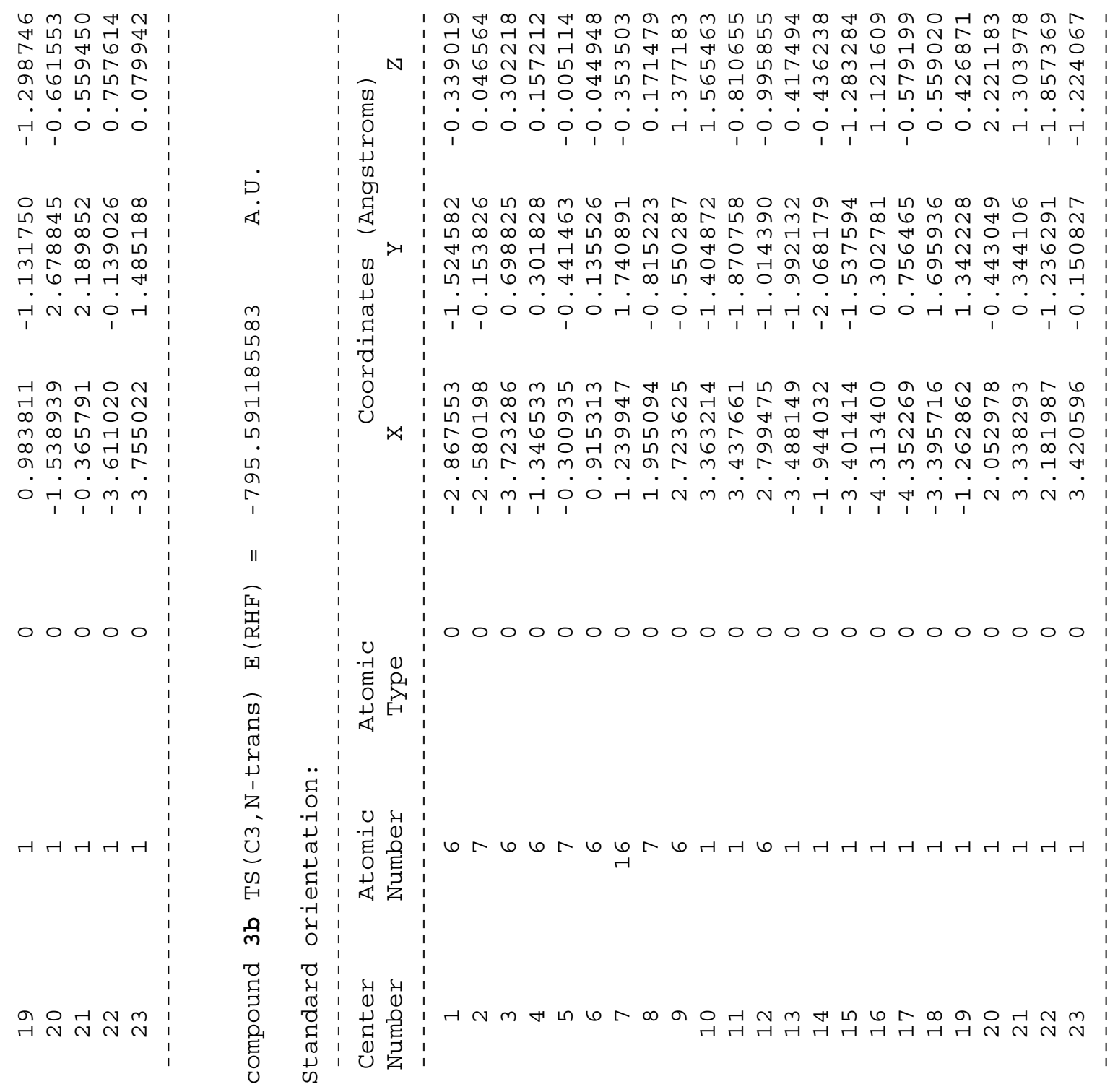




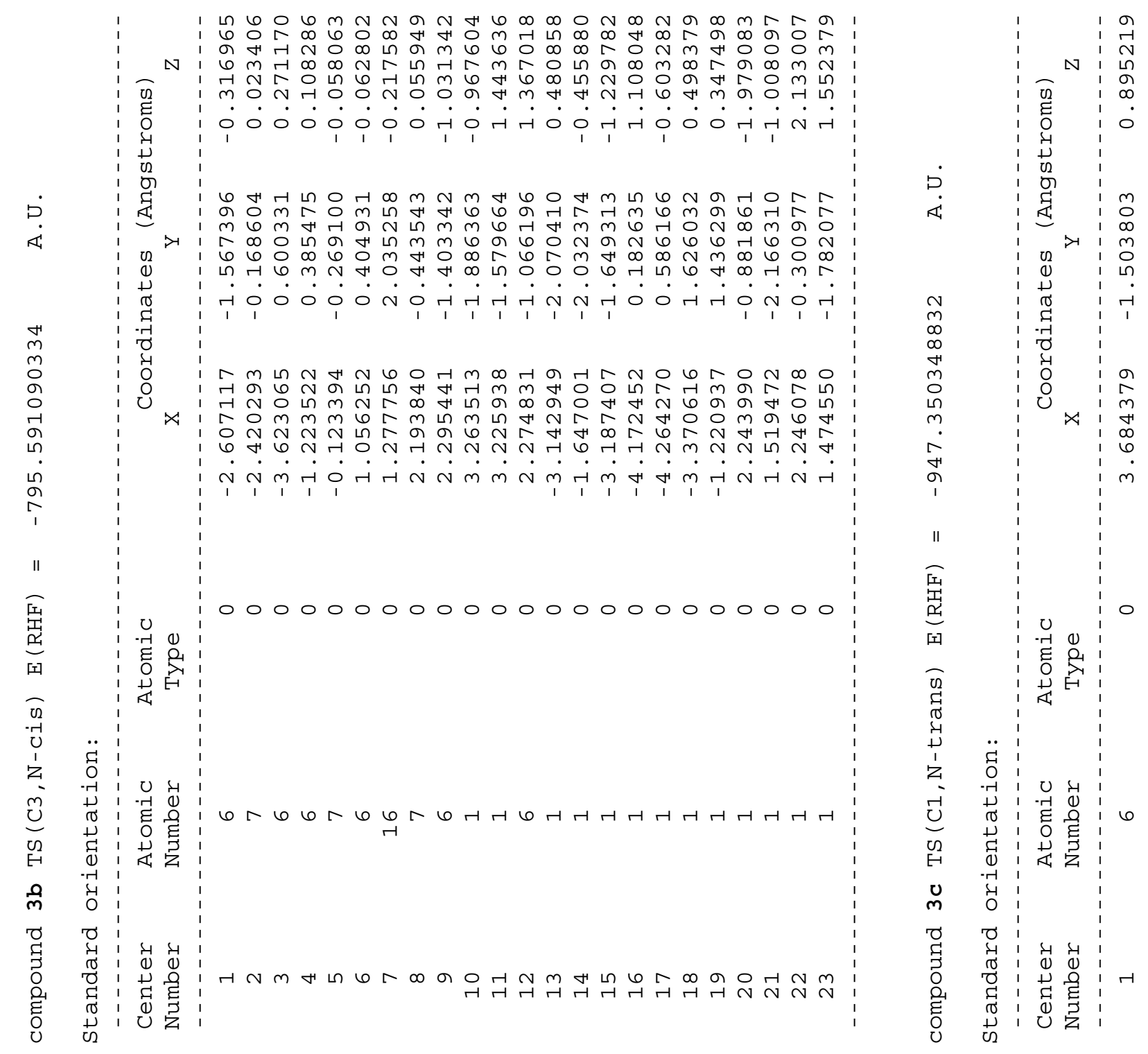


$\infty m \circ m$ m

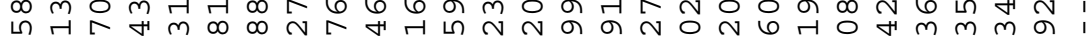

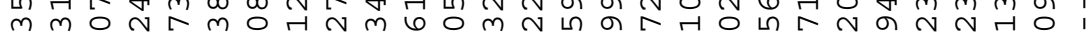

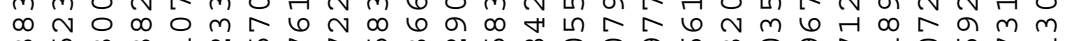

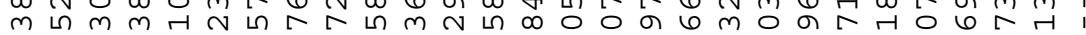

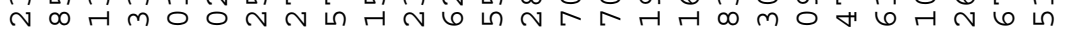

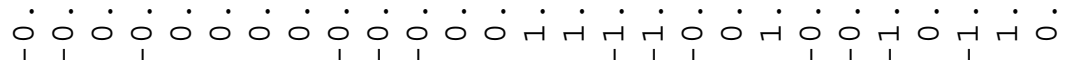

К

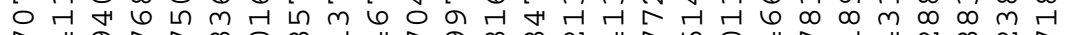
A

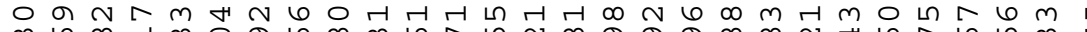

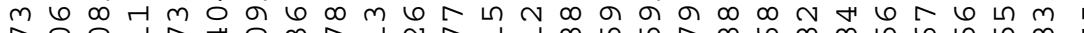
N 0 H O

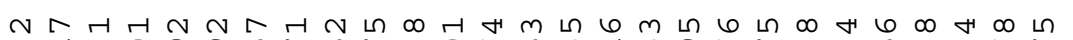

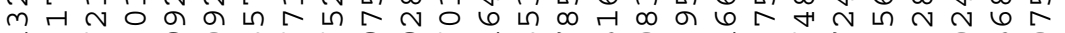

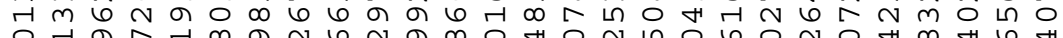
잉

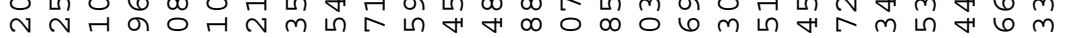

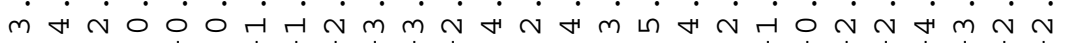
000000000000000000000000000 ^ 6 ช N m

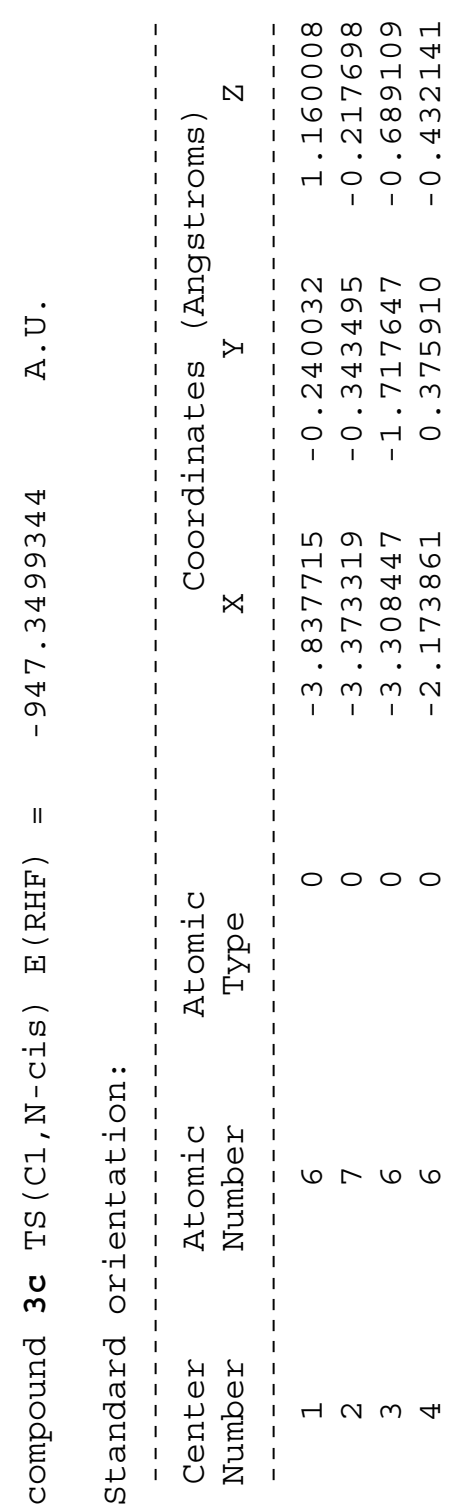




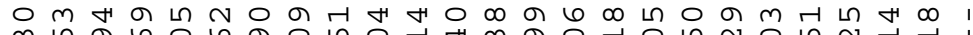

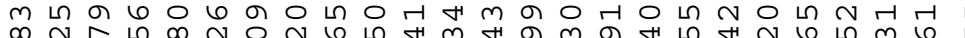

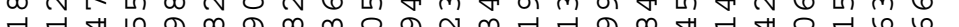
각 H 000 H

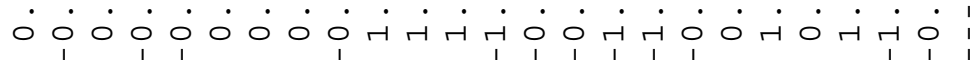

a $N$ t Hos $n$ H

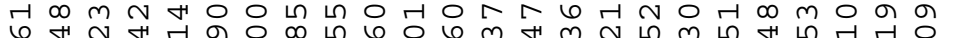

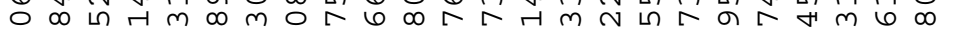

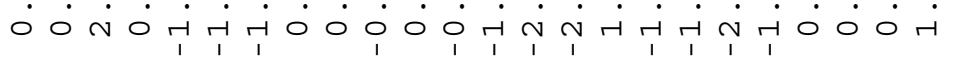

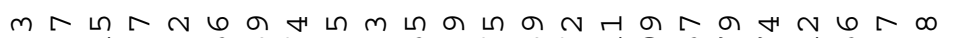

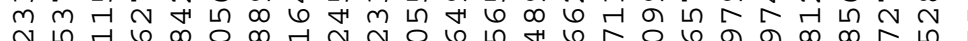
$N$ L ॠँ

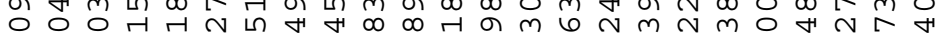

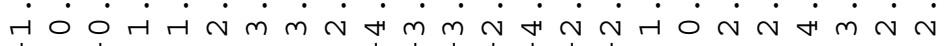

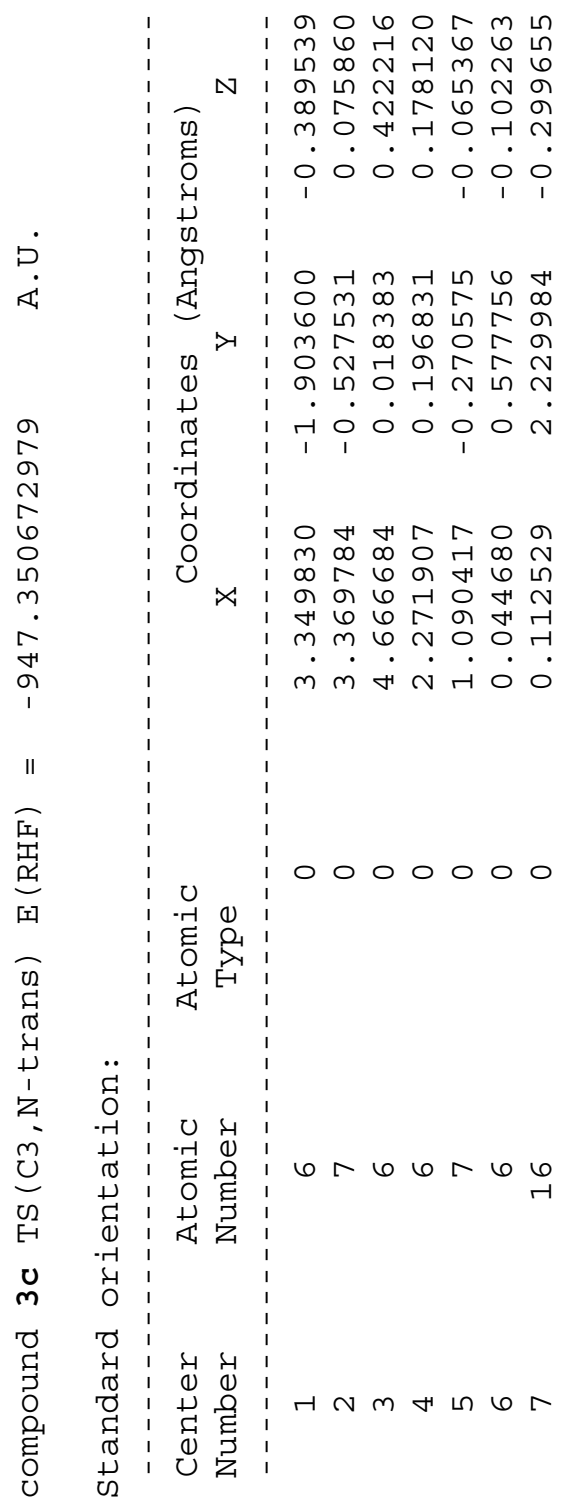




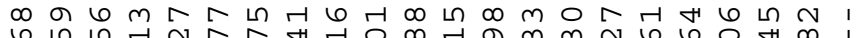
అ

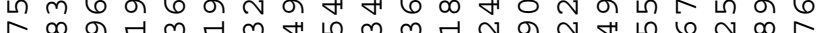
$\hat{0}$ の

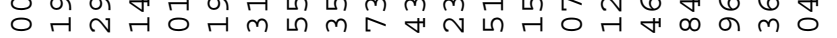

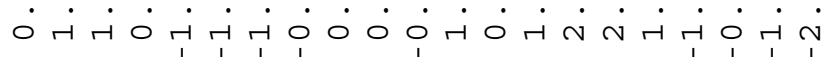

$\infty 6$ म

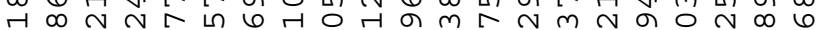

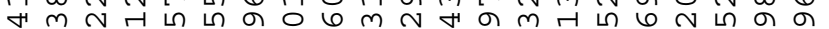

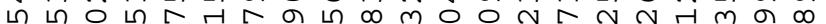

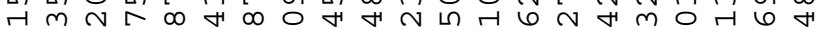

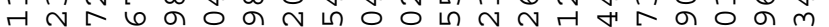

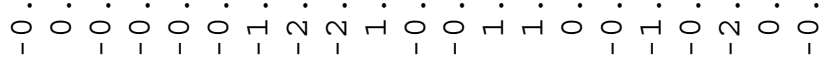

$\infty$ h $N$ L $N$ m $00 \%$ H 0 H

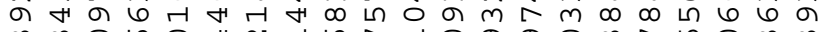

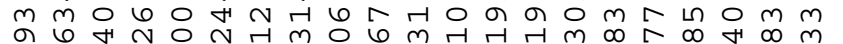

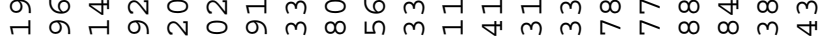

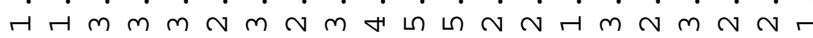
000000000000000000000 $\lambda$. の

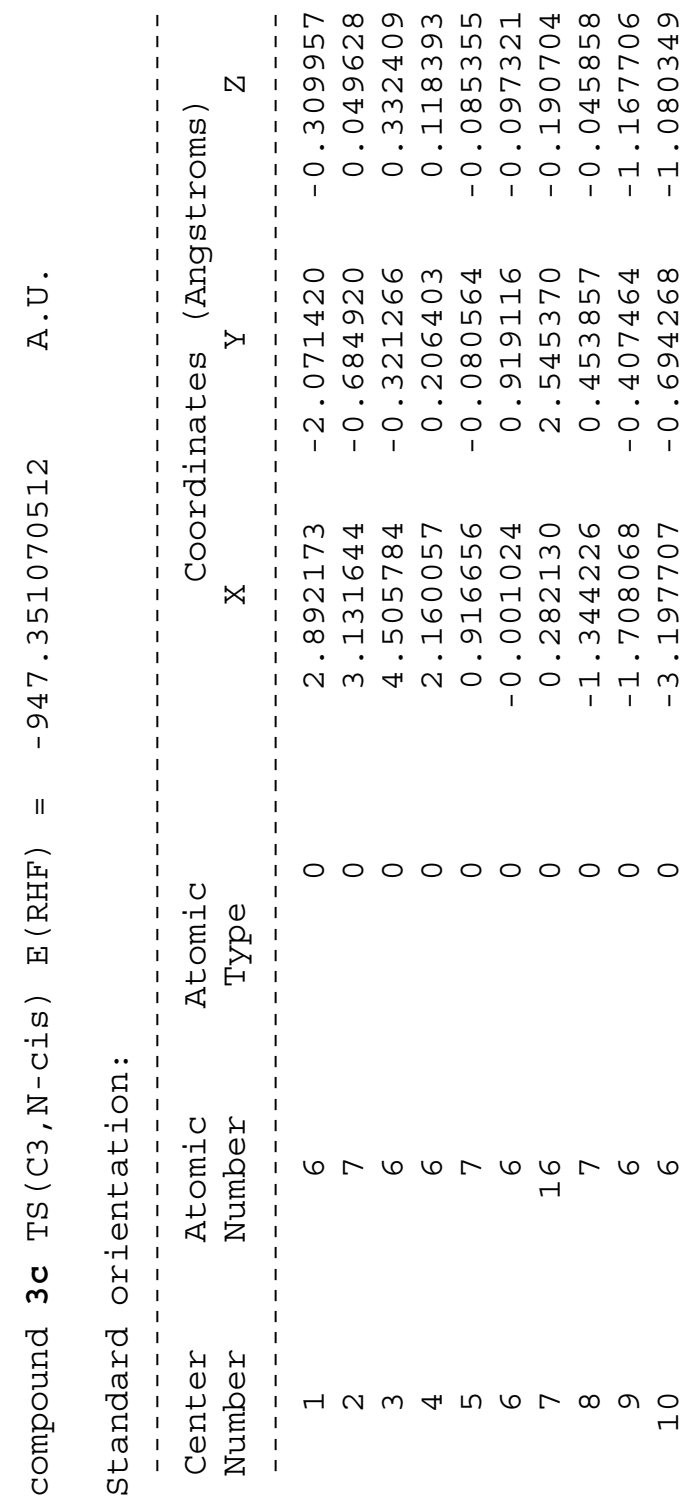


$6 \infty m$ の

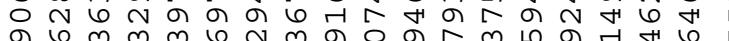

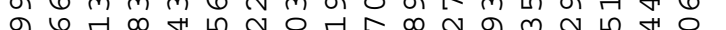
ॠ HN N $N$ H

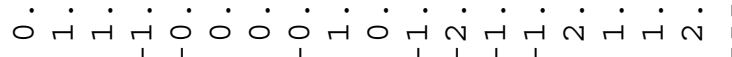

6 の 6 ก

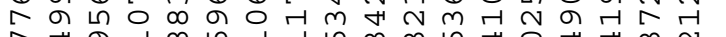

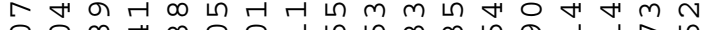
$\circ \circ \infty \quad+m \circ 0$ 대

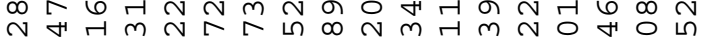

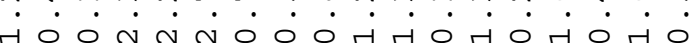

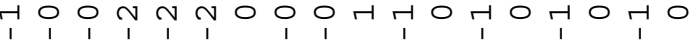

ก $\infty$ N

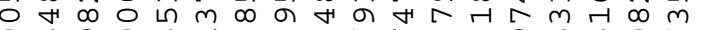

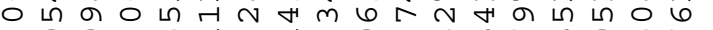

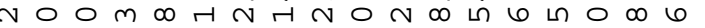

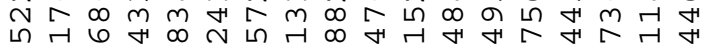

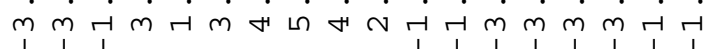

000000000000000000

$\infty 6,4$ 0 -

Hत

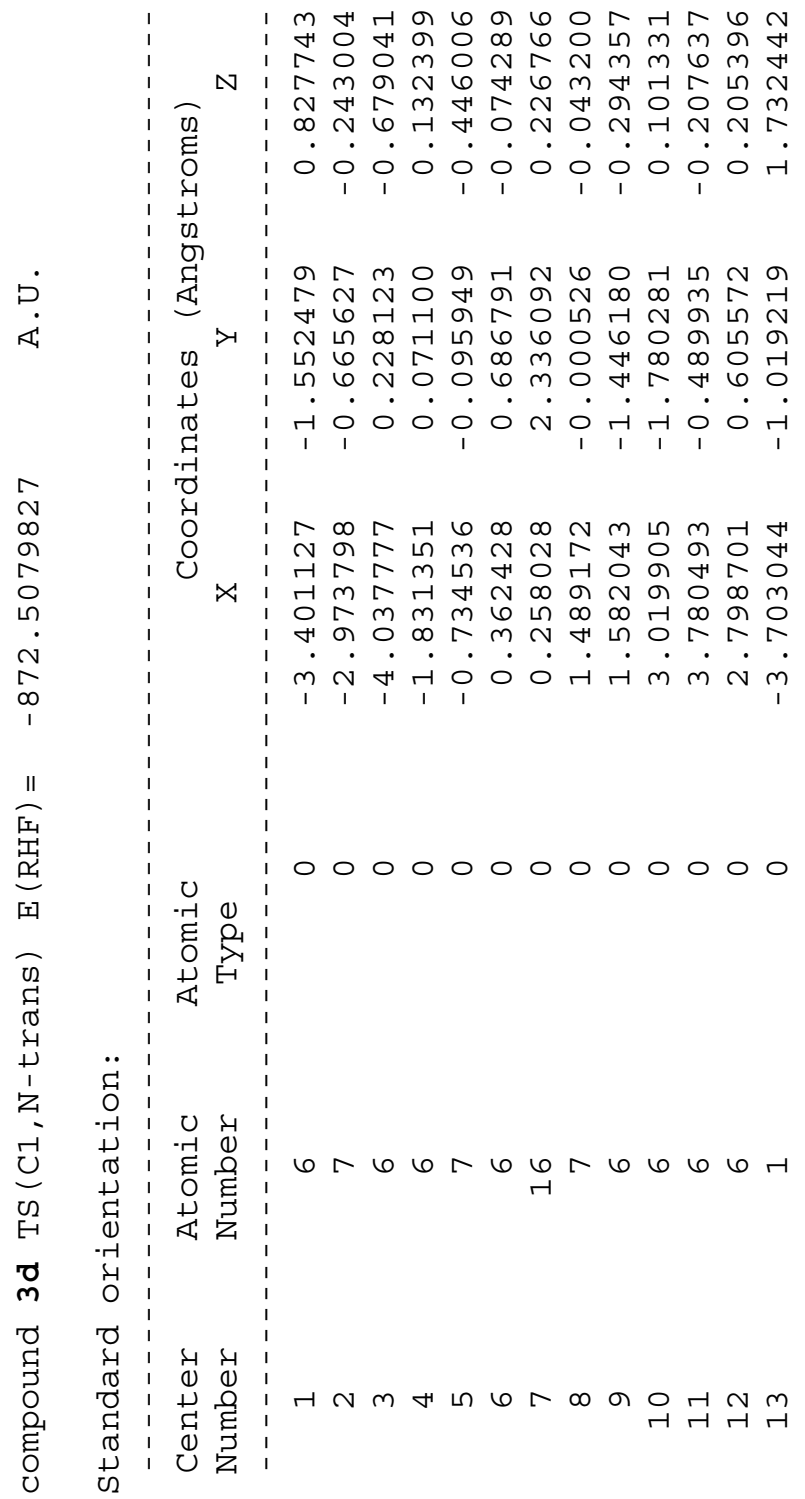


$4 N \Lambda$ H

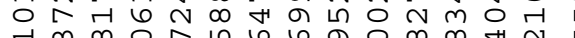
तु

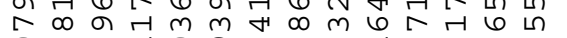
0 H

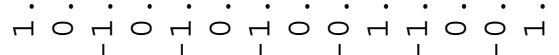

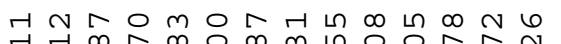

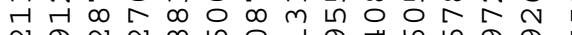

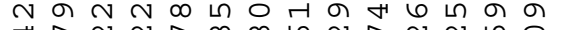

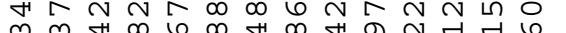

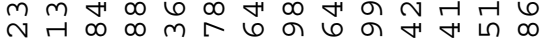

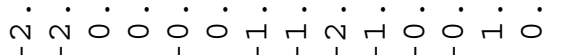

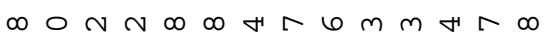
H $\infty$ ब $N$ N

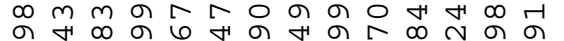

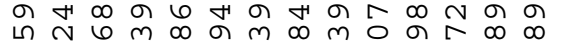

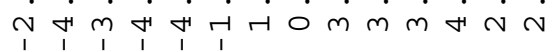

00000000000000

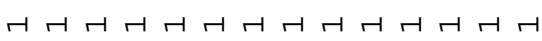

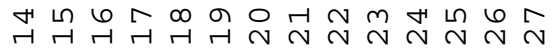

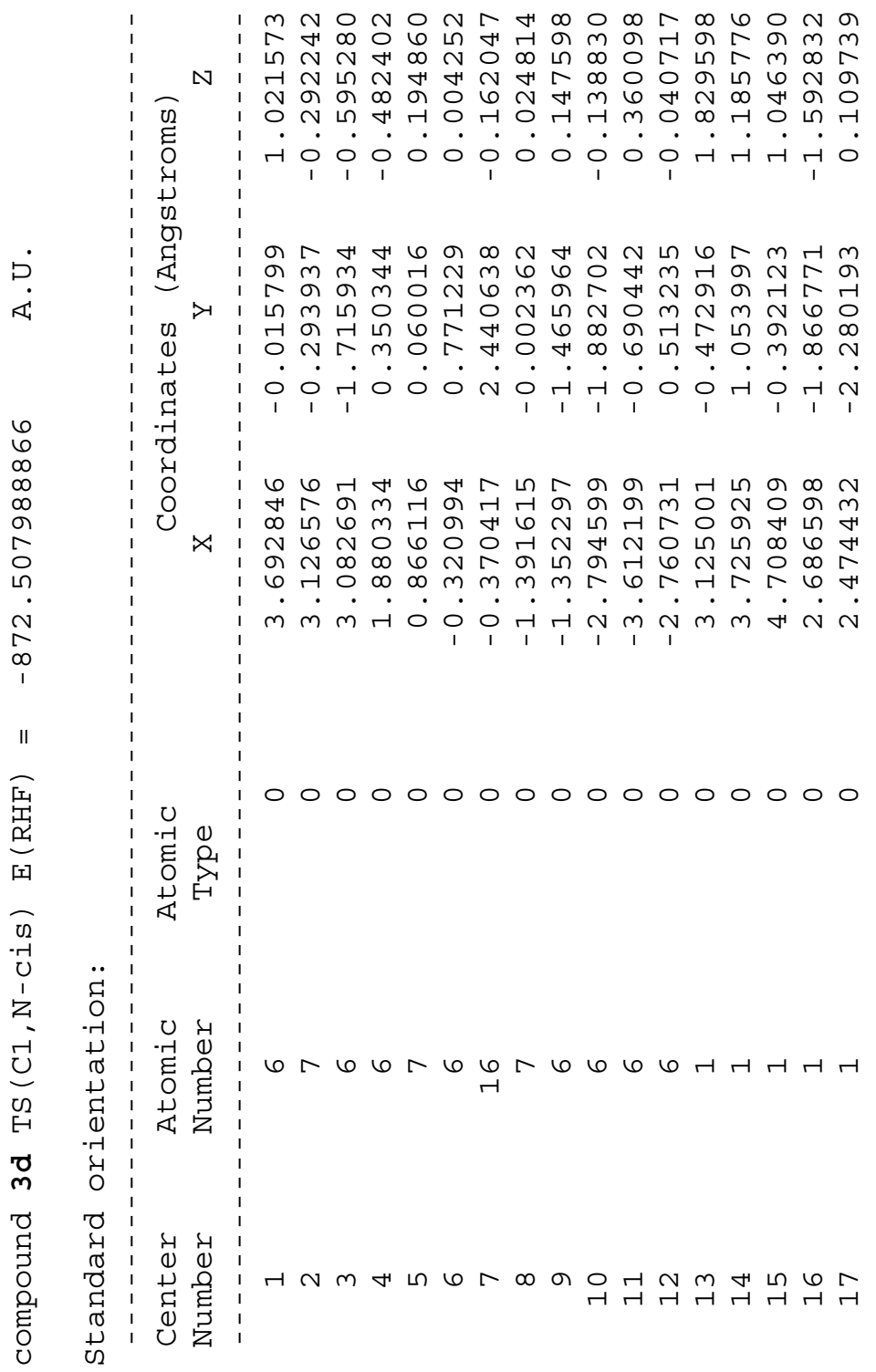

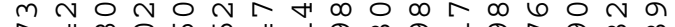
i

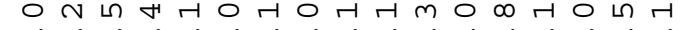
$\dot{0} \dot{0} \dot{0} \dot{0} \dot{0} \dot{0} \dot{0} \dot{0} \dot{0} \dot{0}$

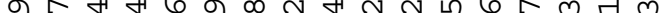

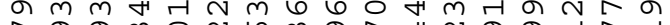
西

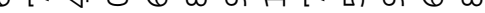

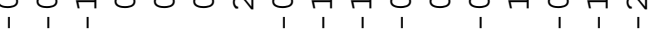

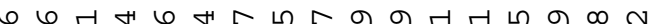
舟

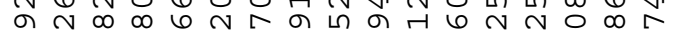
6 - $000 \mathrm{mmmm} 6$ m $m m m-000 H H N m \sim m m$ H N

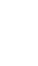




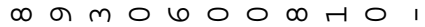

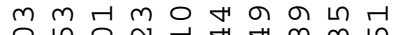
0 ก

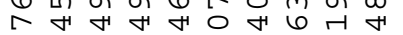
เ $N$ म $\mathrm{m} N$ + 060

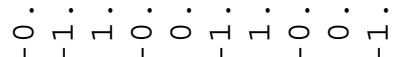

$m$ の નુ

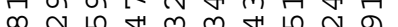
म 0 म

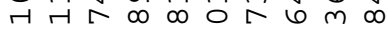

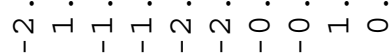

H T O M m $0 \mathrm{~N}$ H

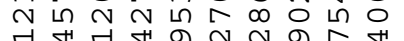
각न

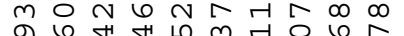

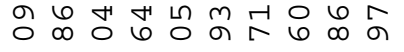
$\dot{\forall} \vec{r} \dot{0} \dot{m} \dot{N} \dot{m} \dot{N}$ 0000000000 $444-44-44$

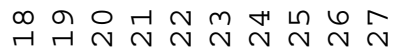

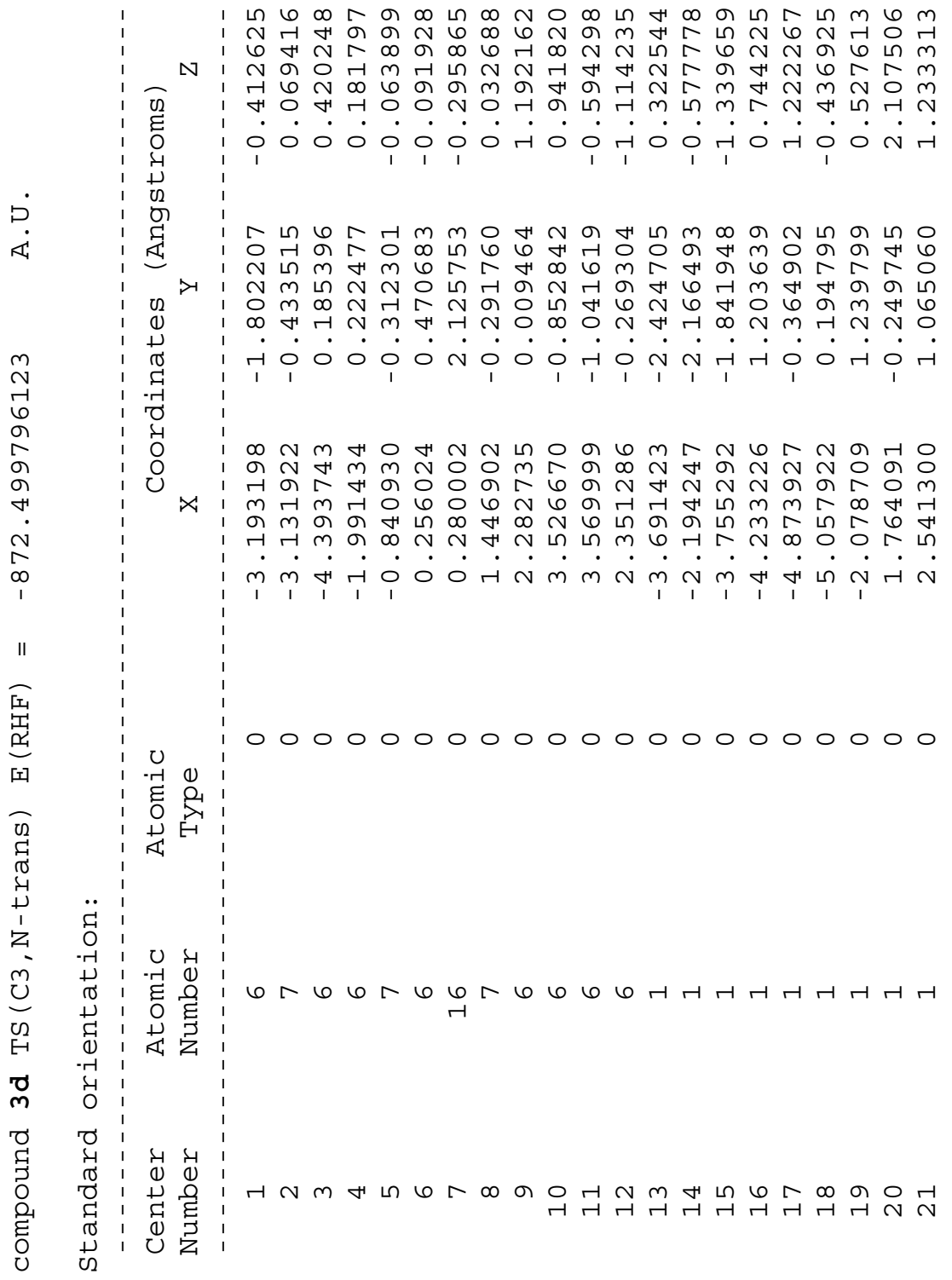

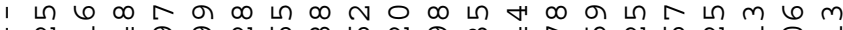
न

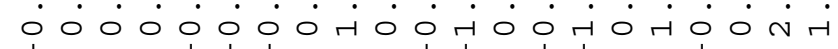

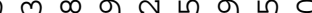

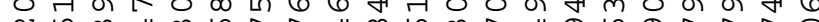

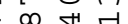
$m m m$

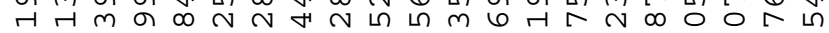

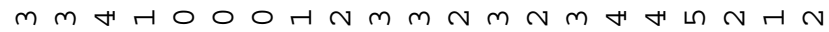
H 


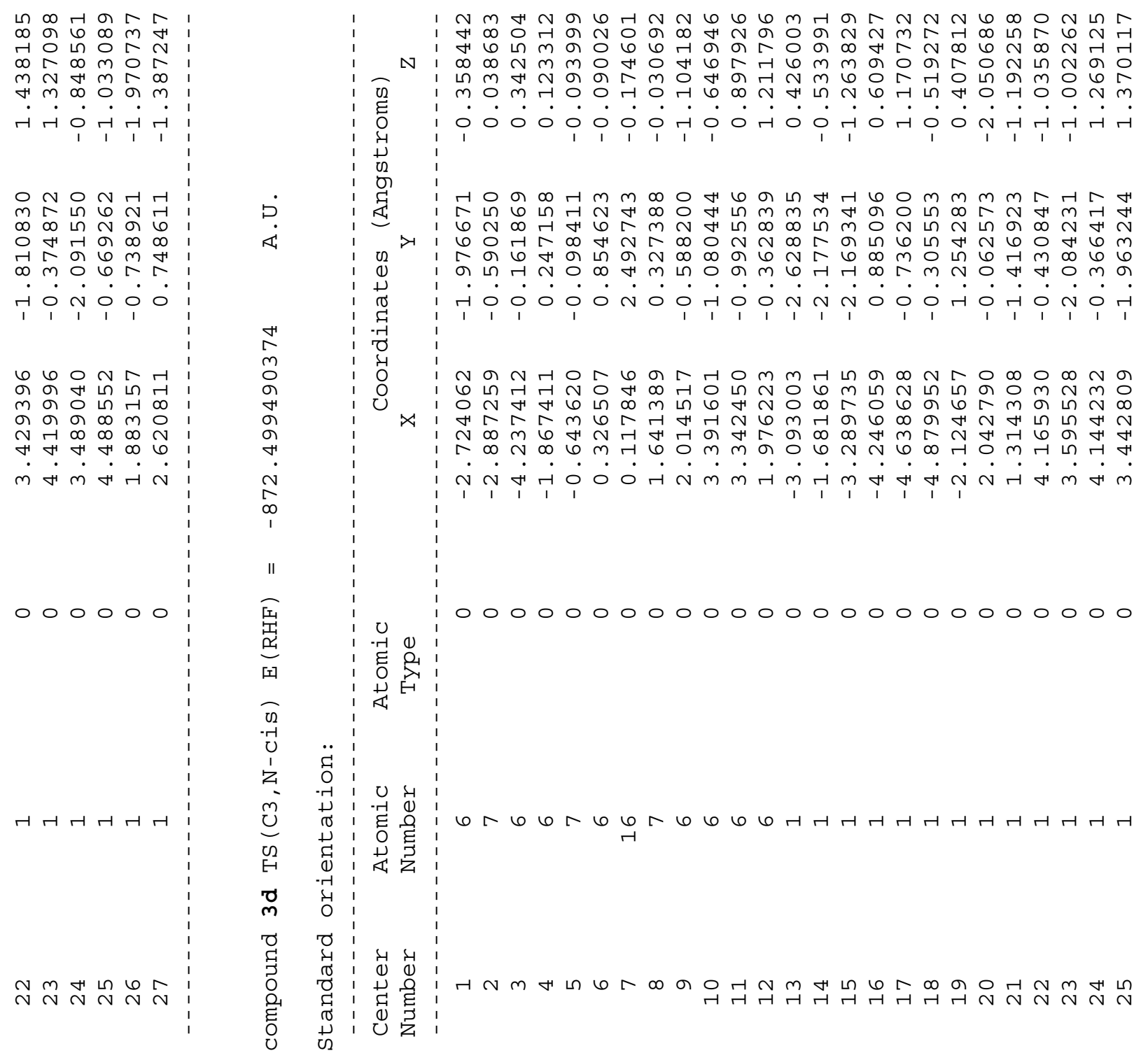




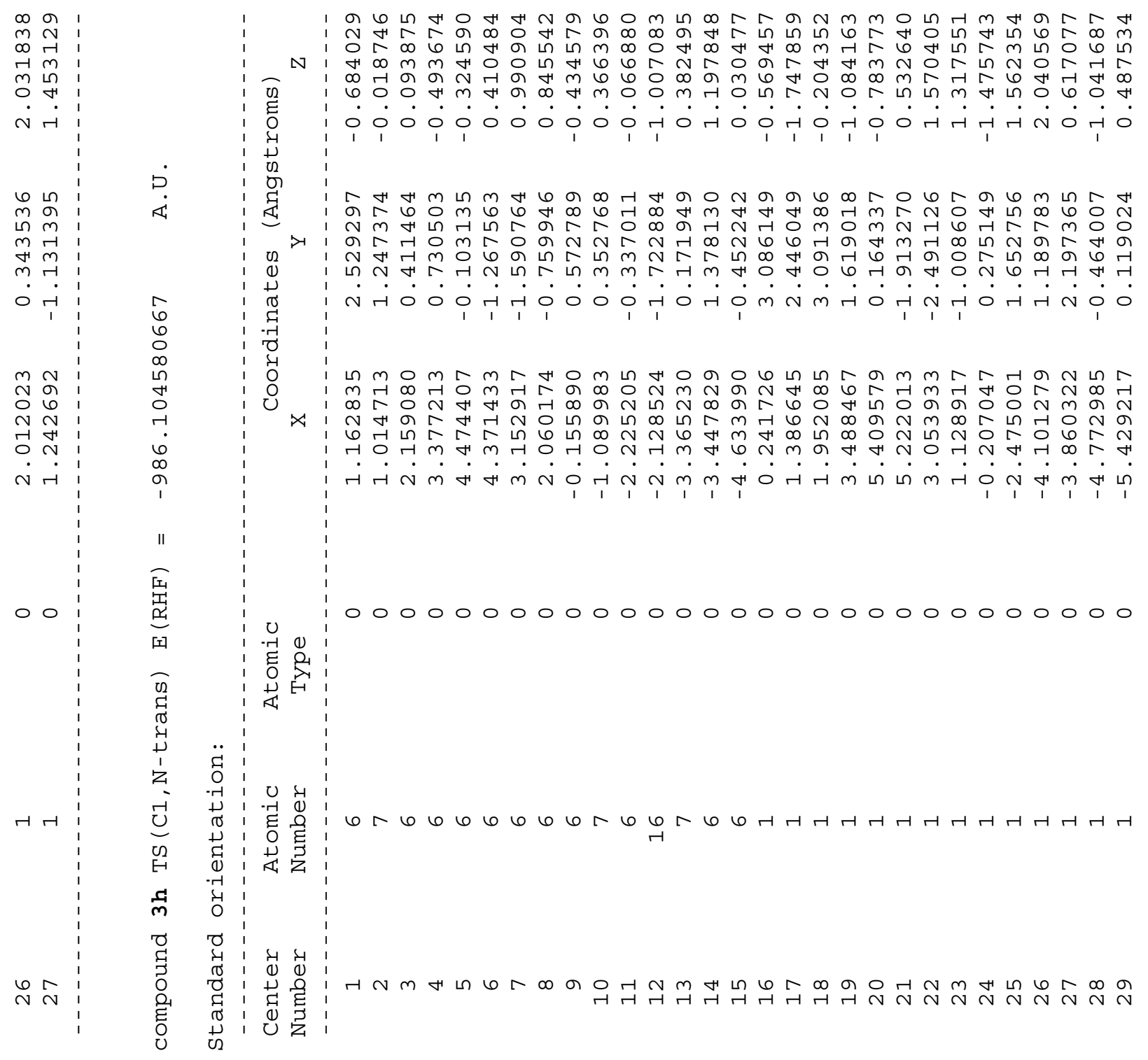




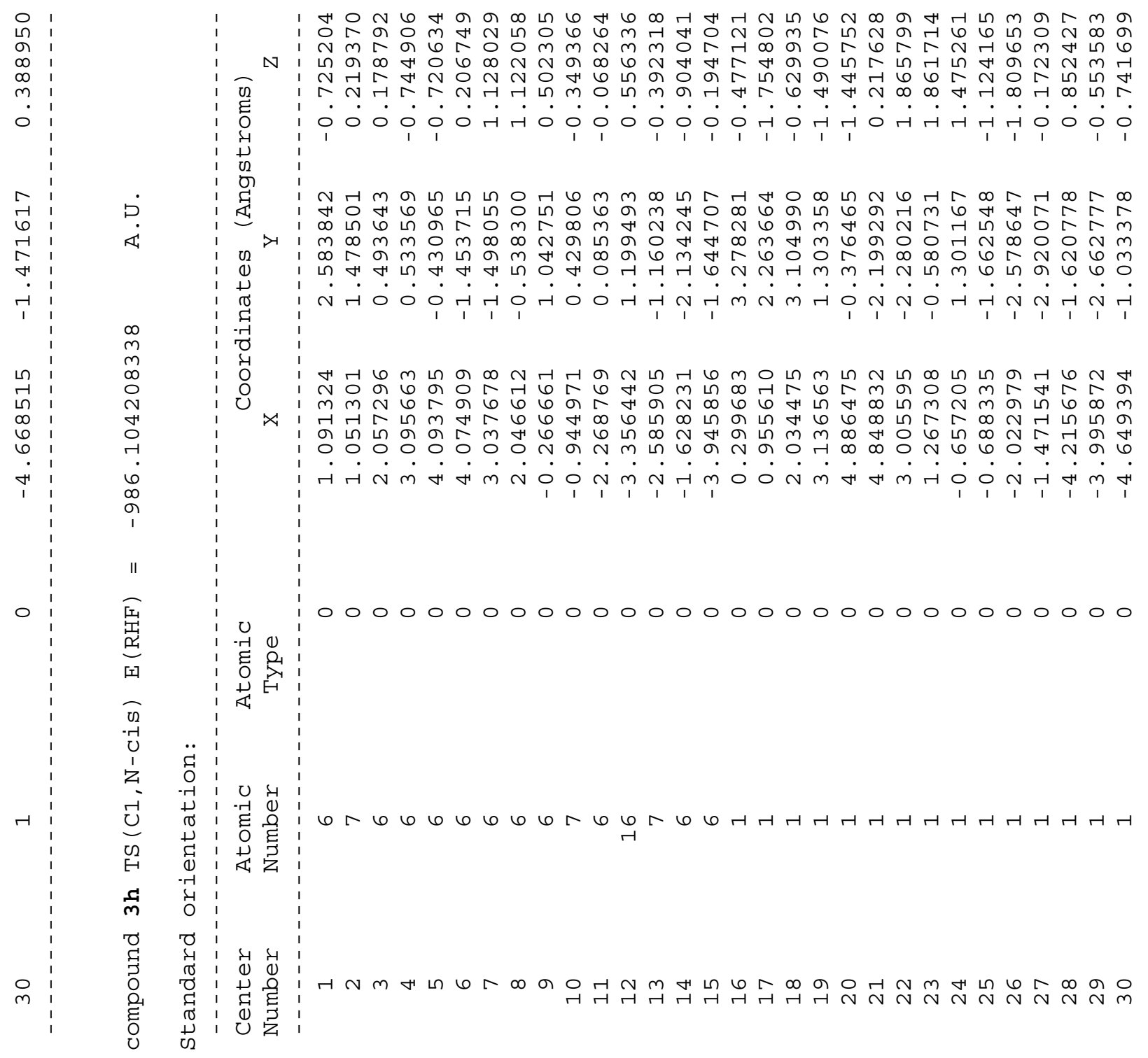




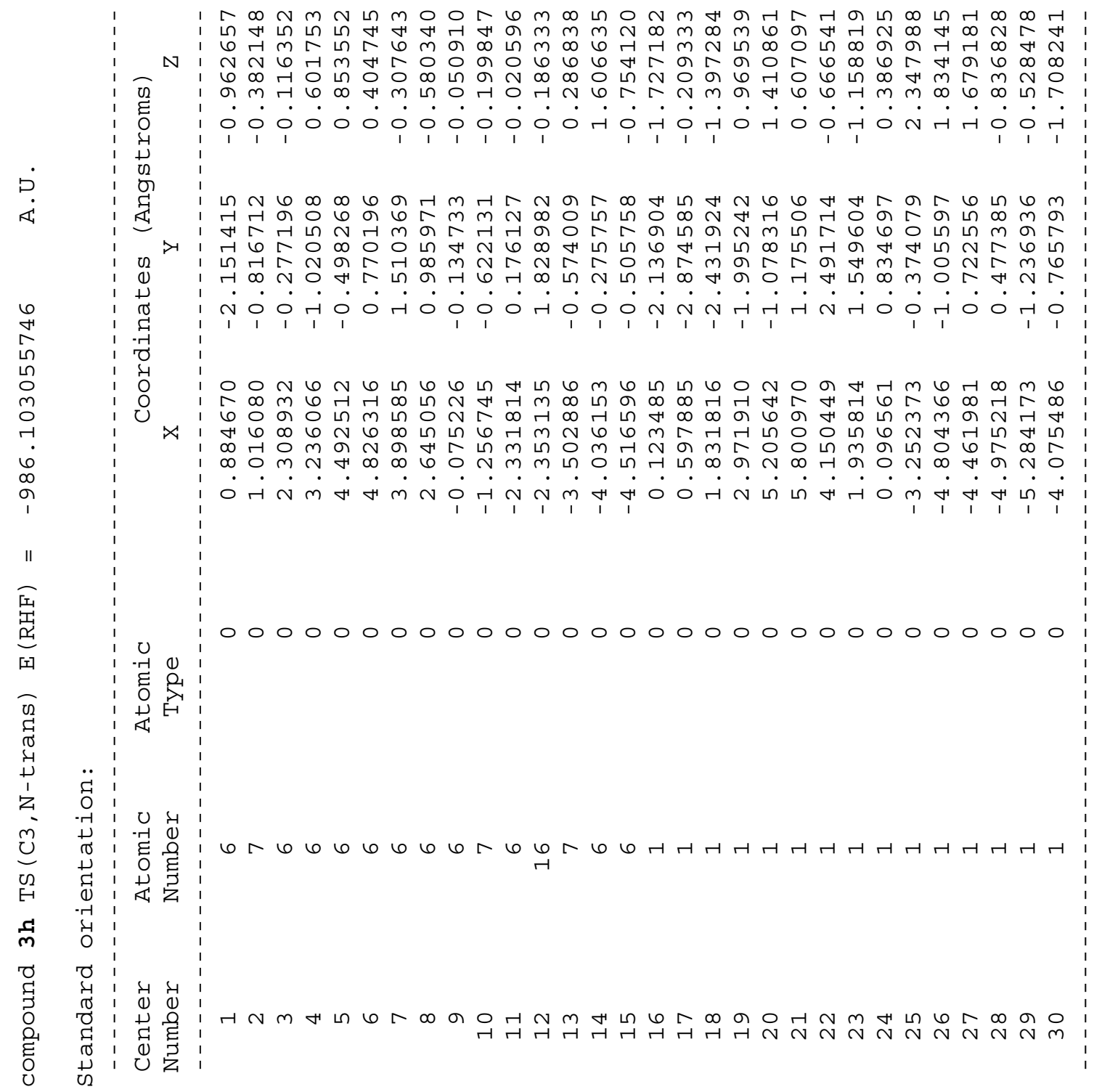




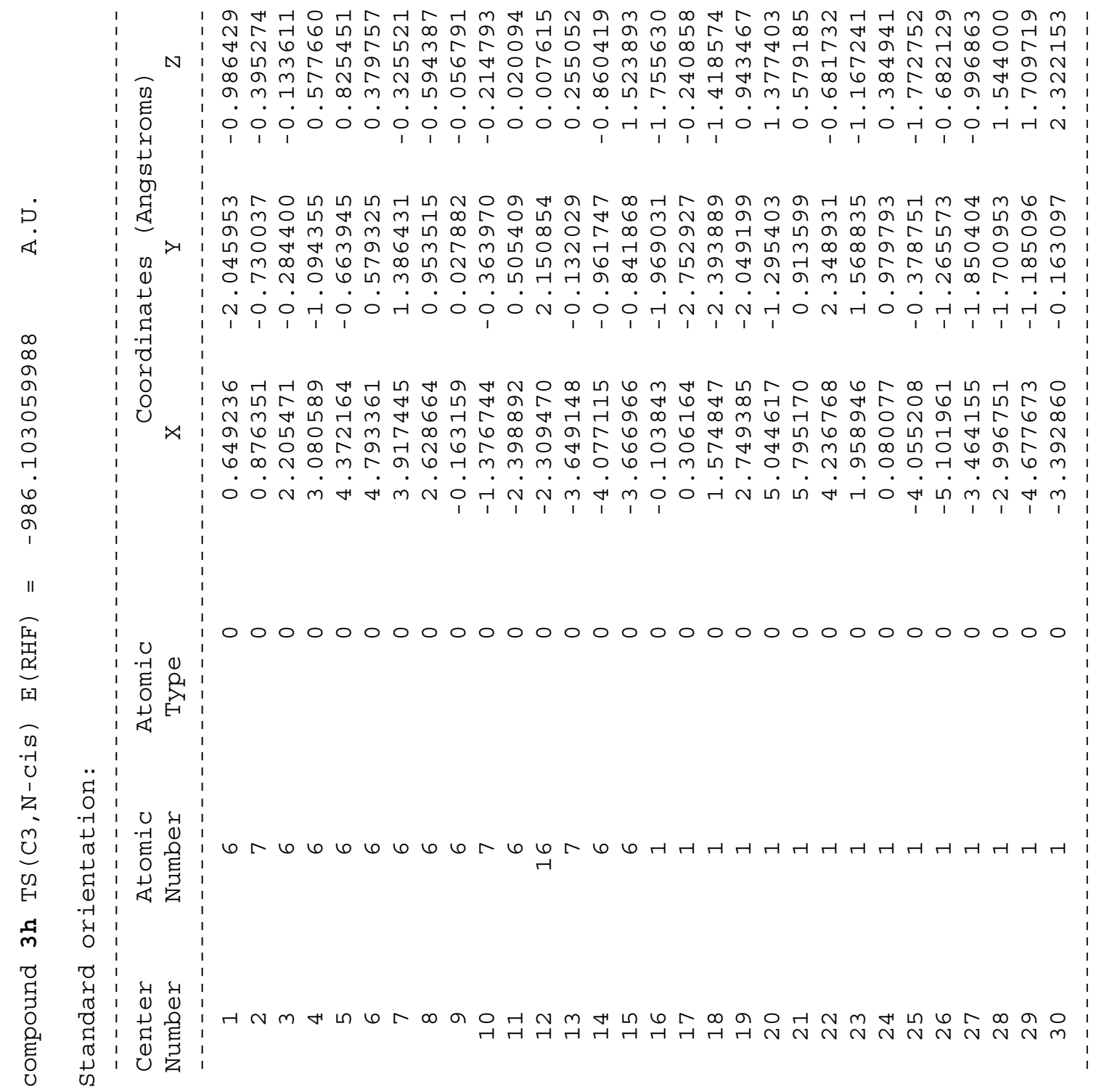




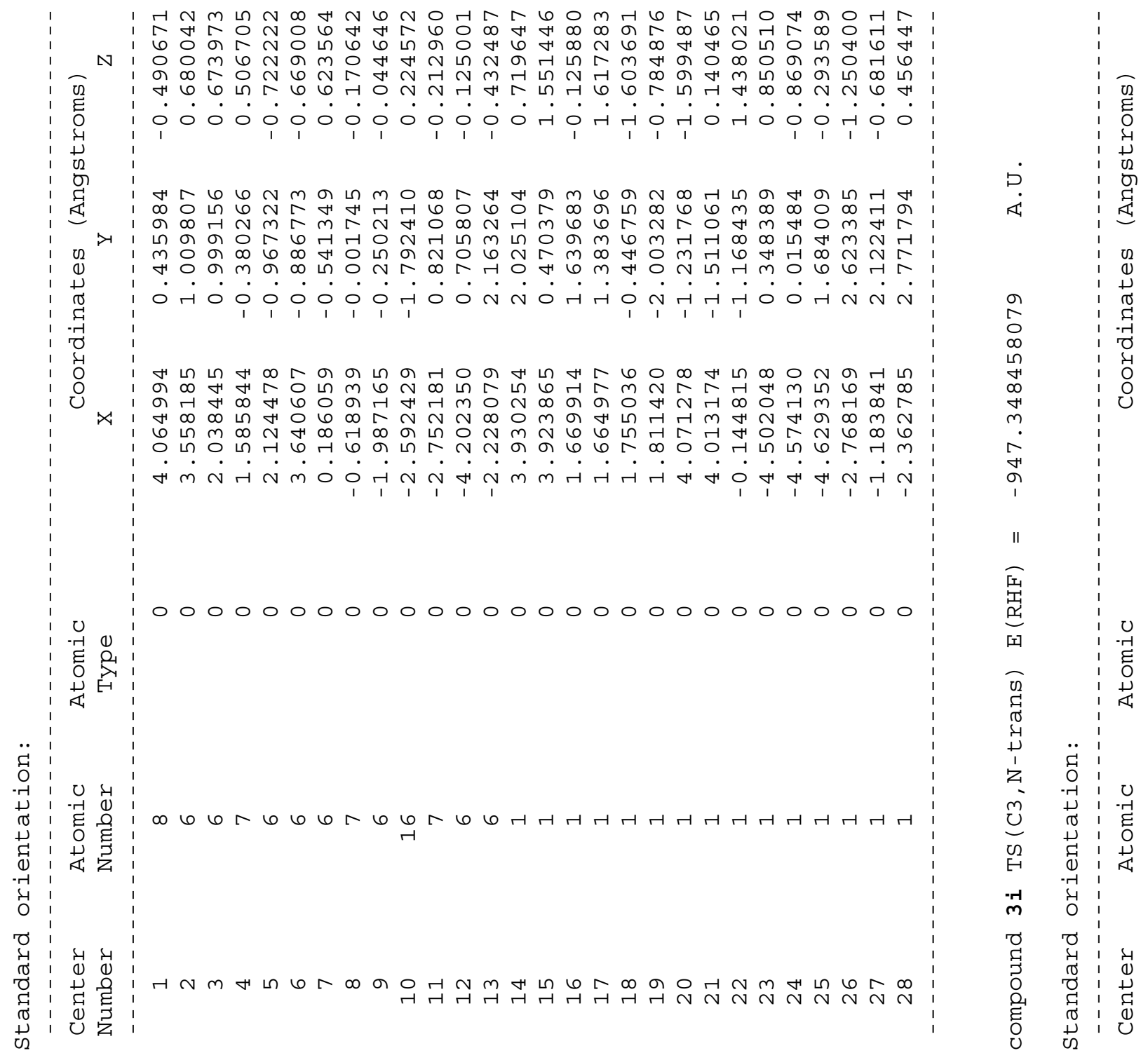


। $\infty \infty$ ம

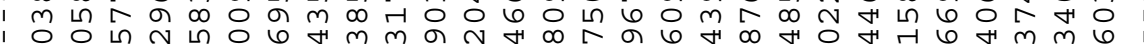

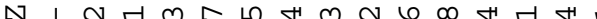

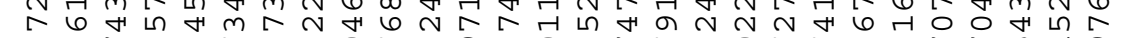

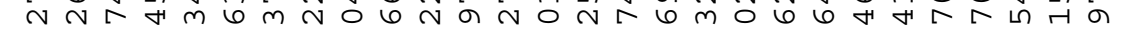

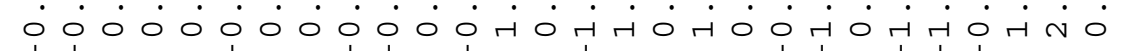

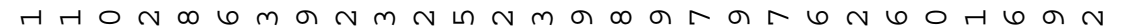
เ

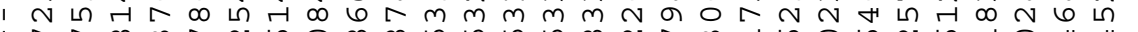

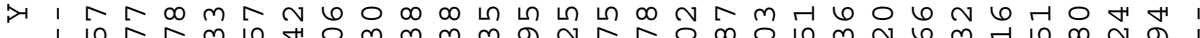

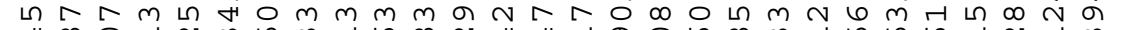

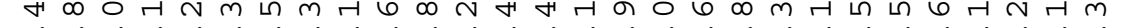

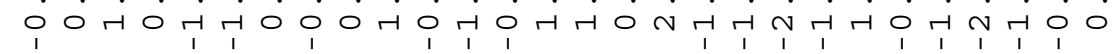

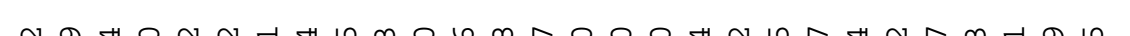
N G H $0 N N$ H ब 6 ब

$x$ r

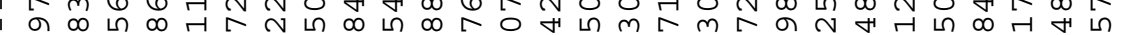

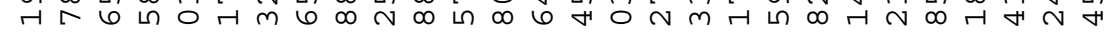

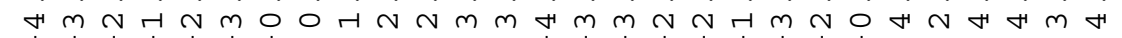<smiles>[Li][Ca][Ca]</smiles>

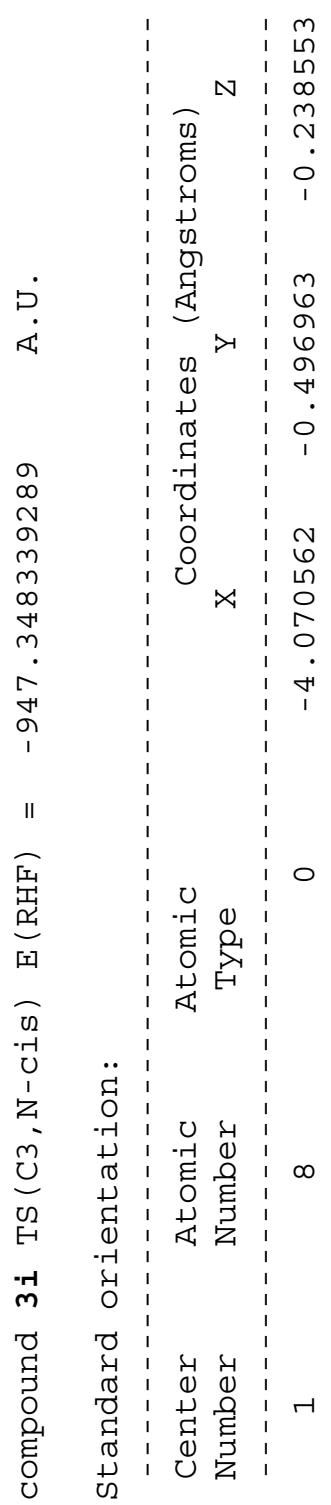




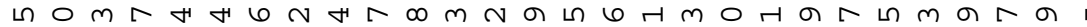

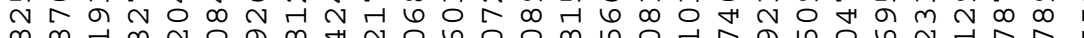

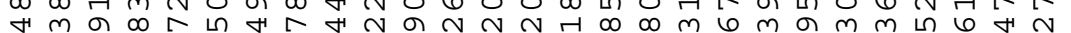

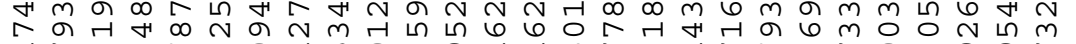

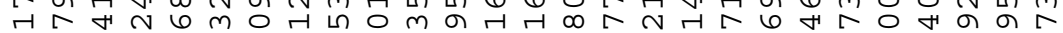

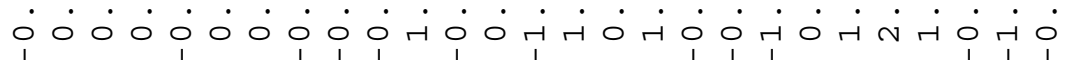

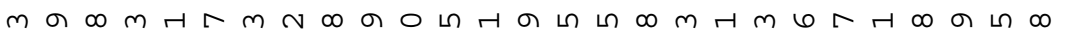
स $m$ म $m$ स

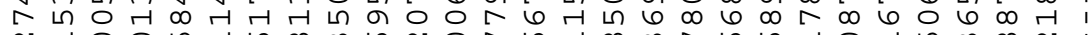
$N$ 다 0 에

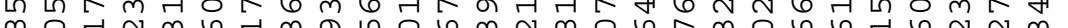

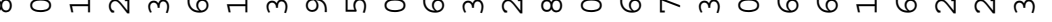

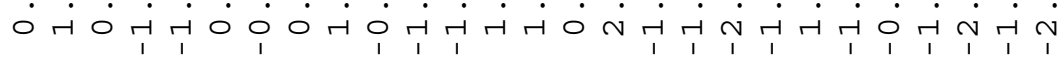

人 ○

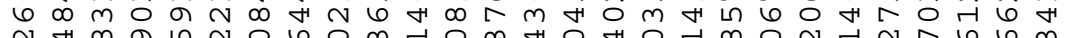
가

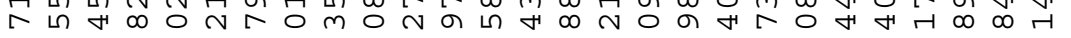

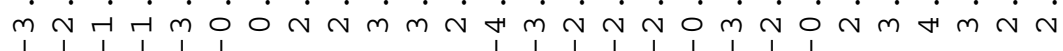

000000000000000000000000000

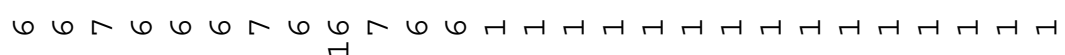

N m

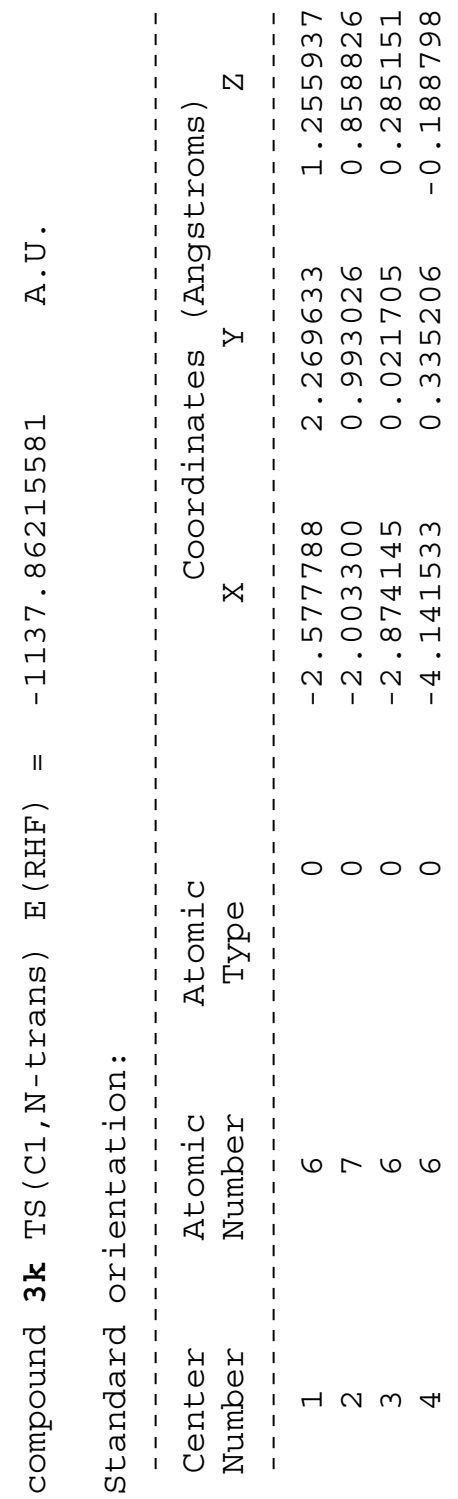


$m m \infty \wedge \circ N 6 m m 6 \infty$ เ

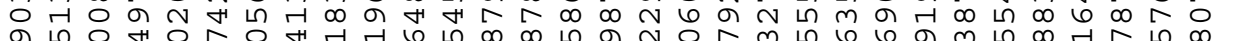

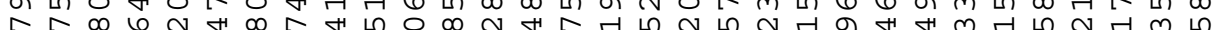

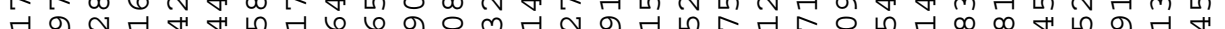

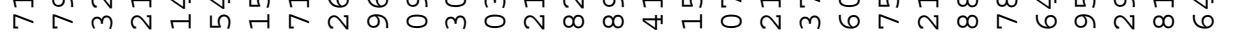

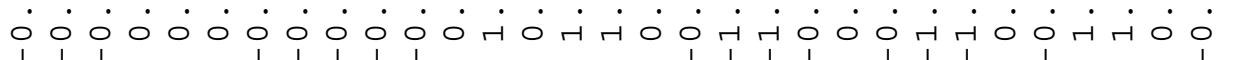
の $\ln 6$ H $\infty$ a mo

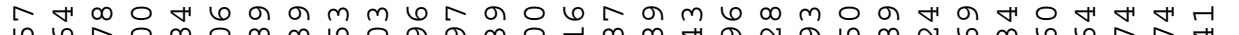

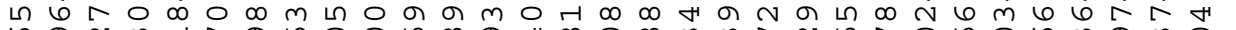
6 ब $\mathrm{m}$ -

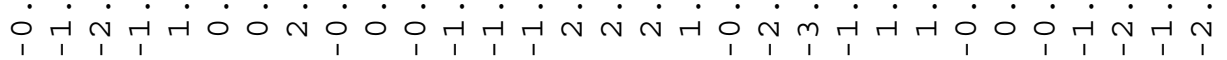

L

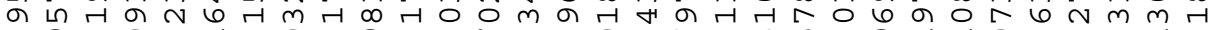

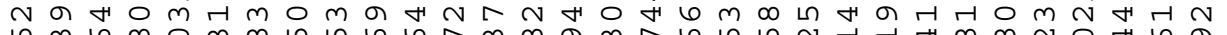

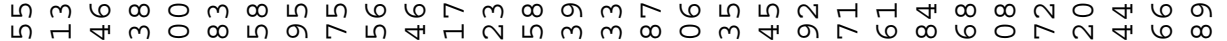

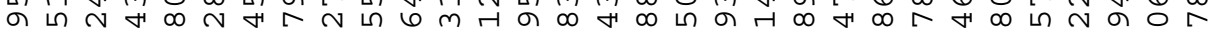

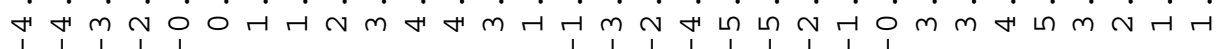
0000000000000000000000000000000 $66666 \wedge 66 \wedge 66066$ 6 6

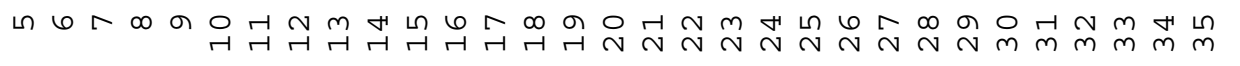

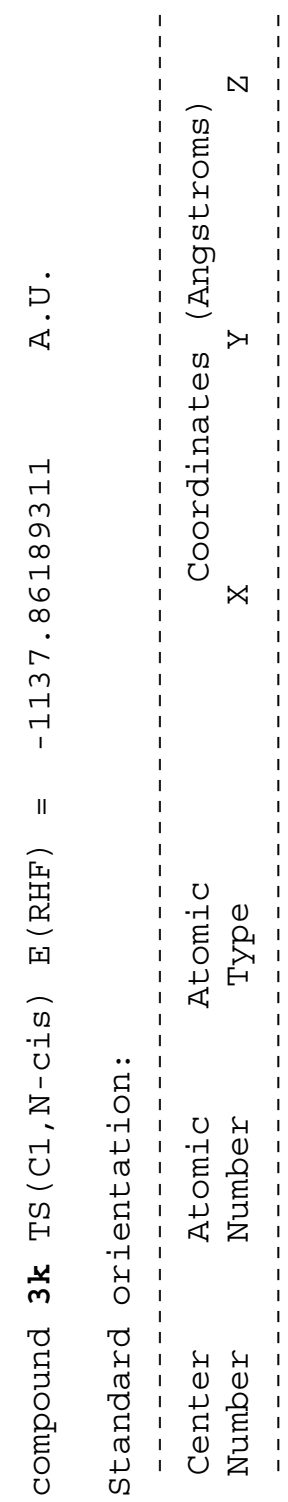




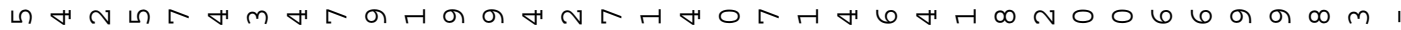

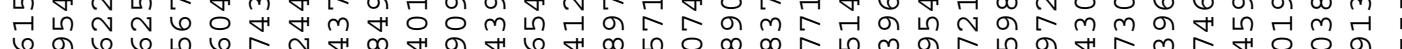
$6000 \%$

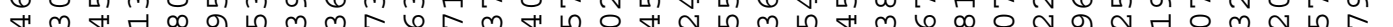

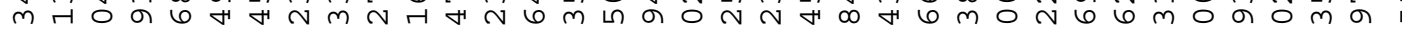

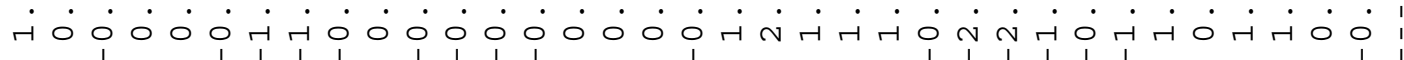

H人 $\infty$ H

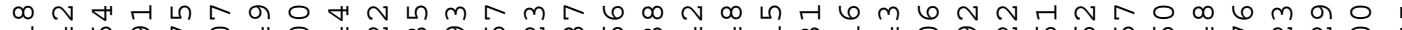
개

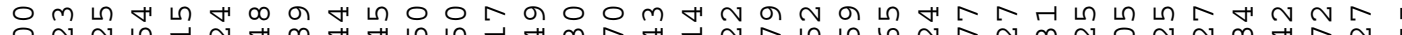

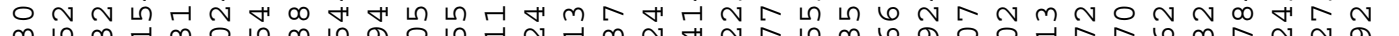

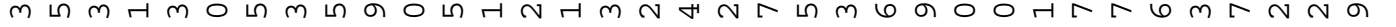

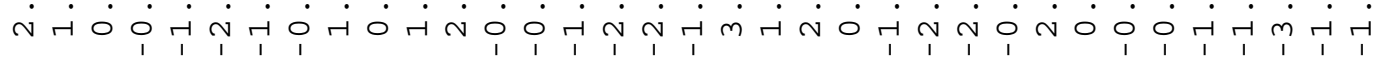

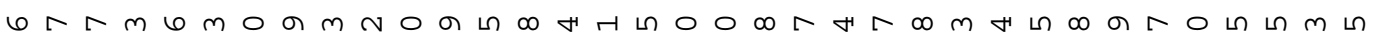
H

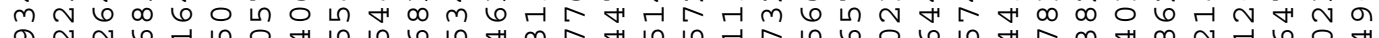

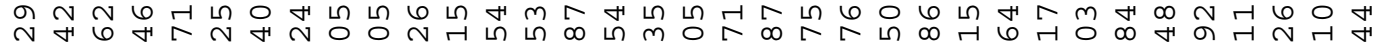

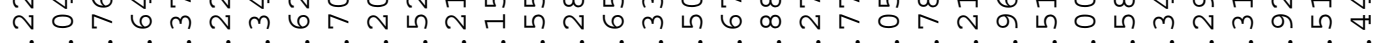

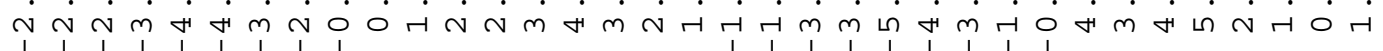

00000000000000000000000000000000000

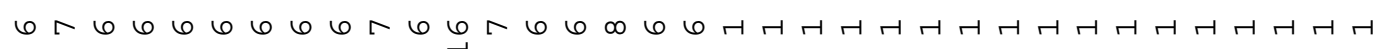
N 


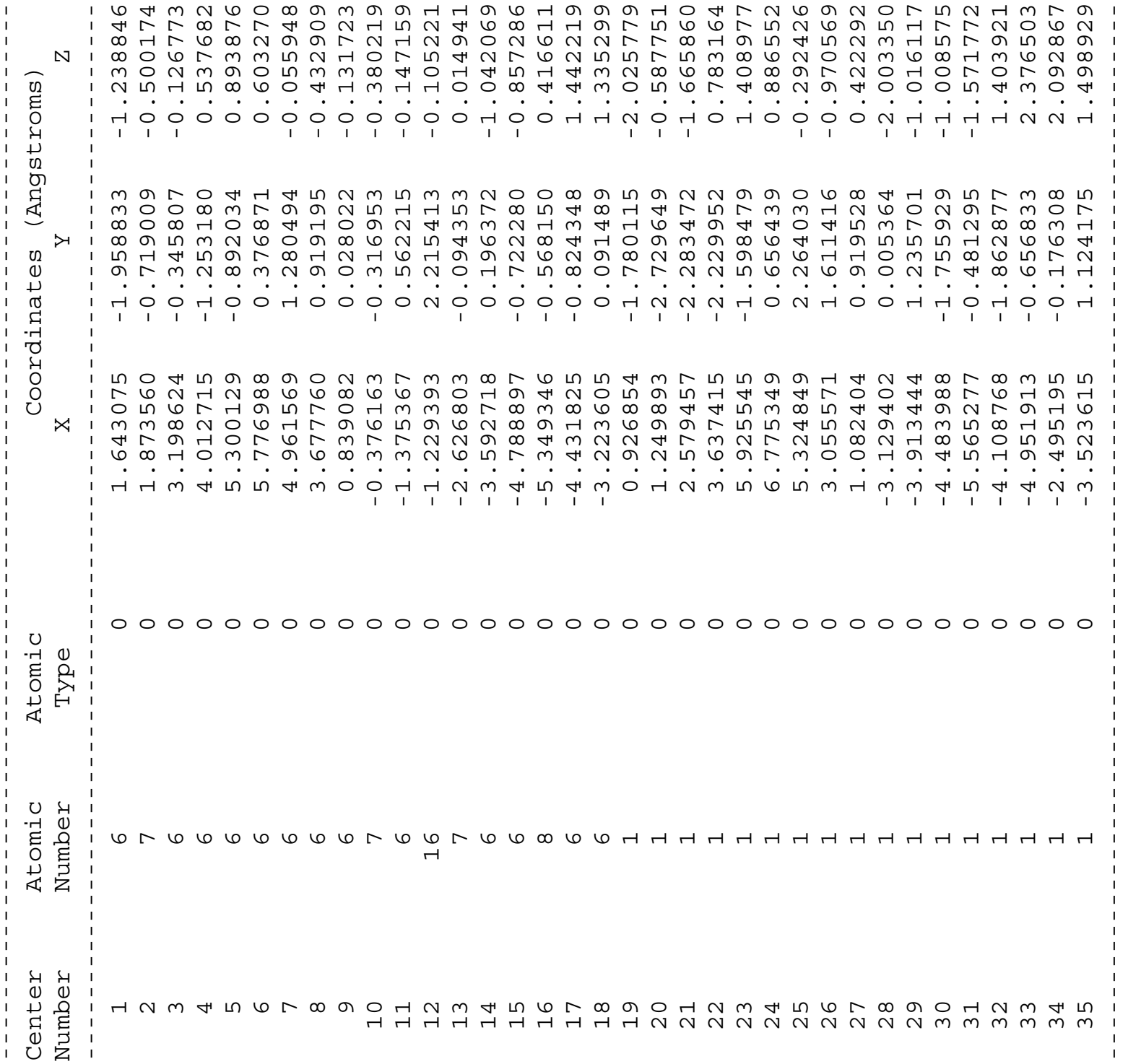




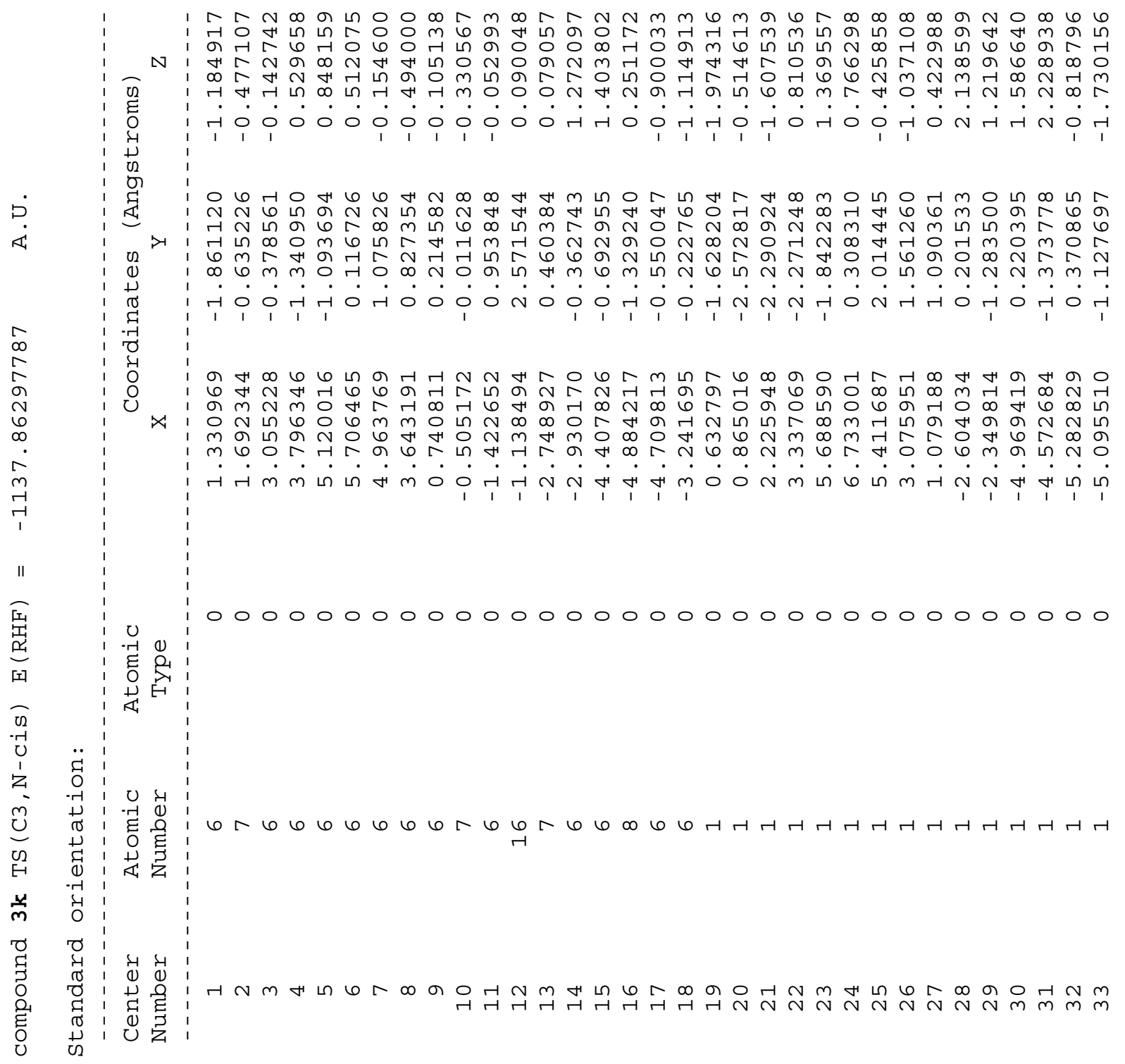




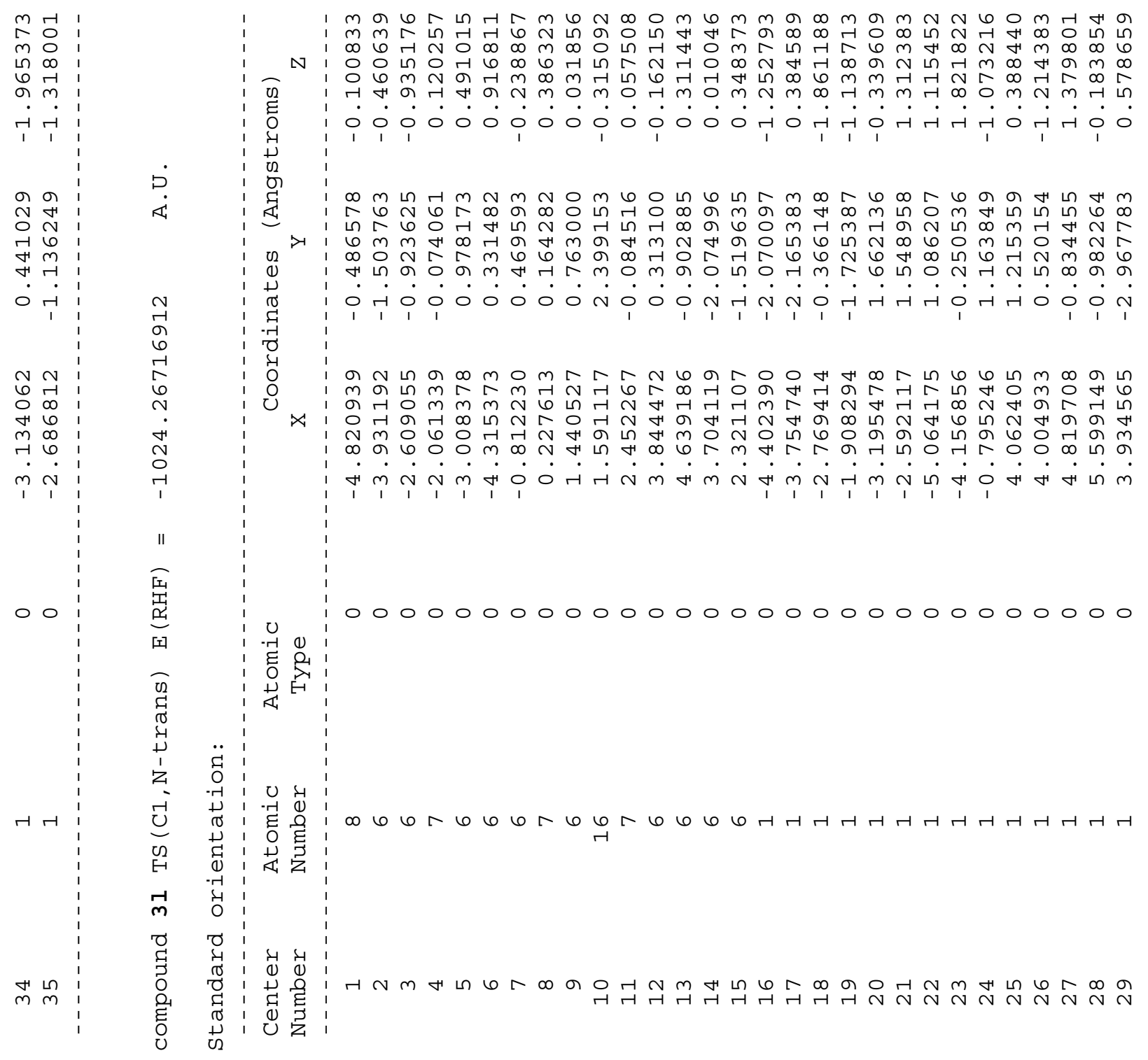




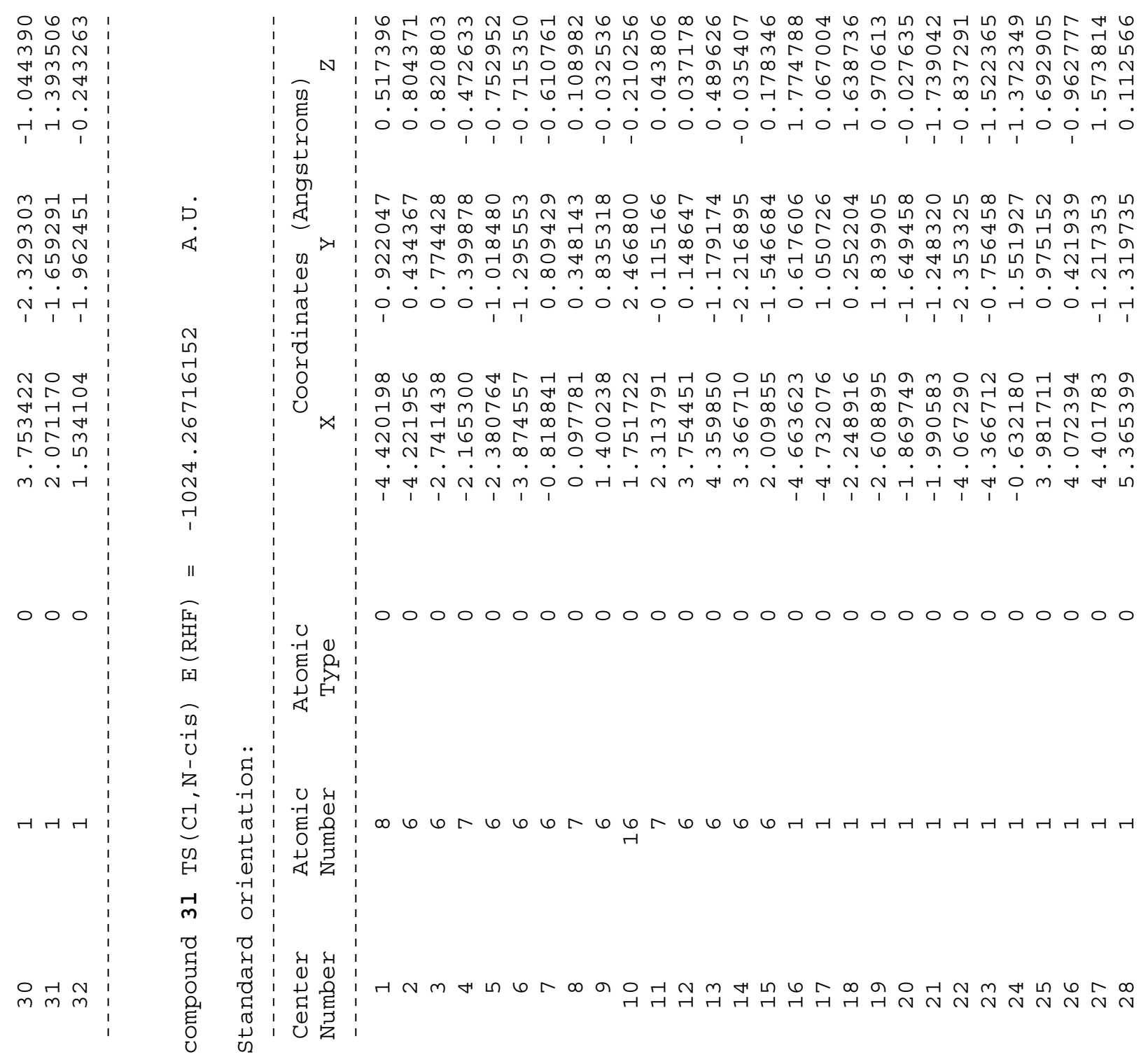




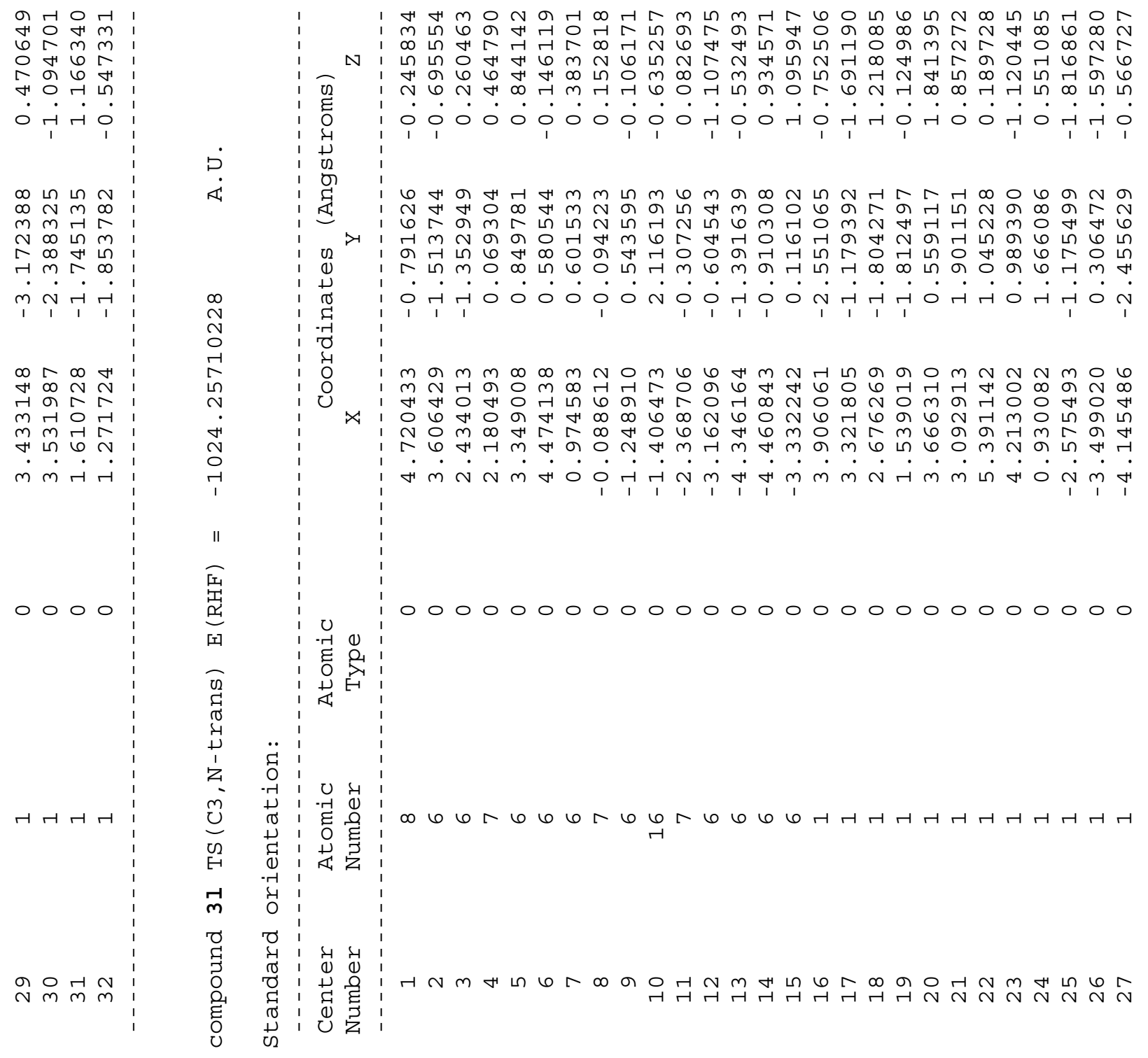




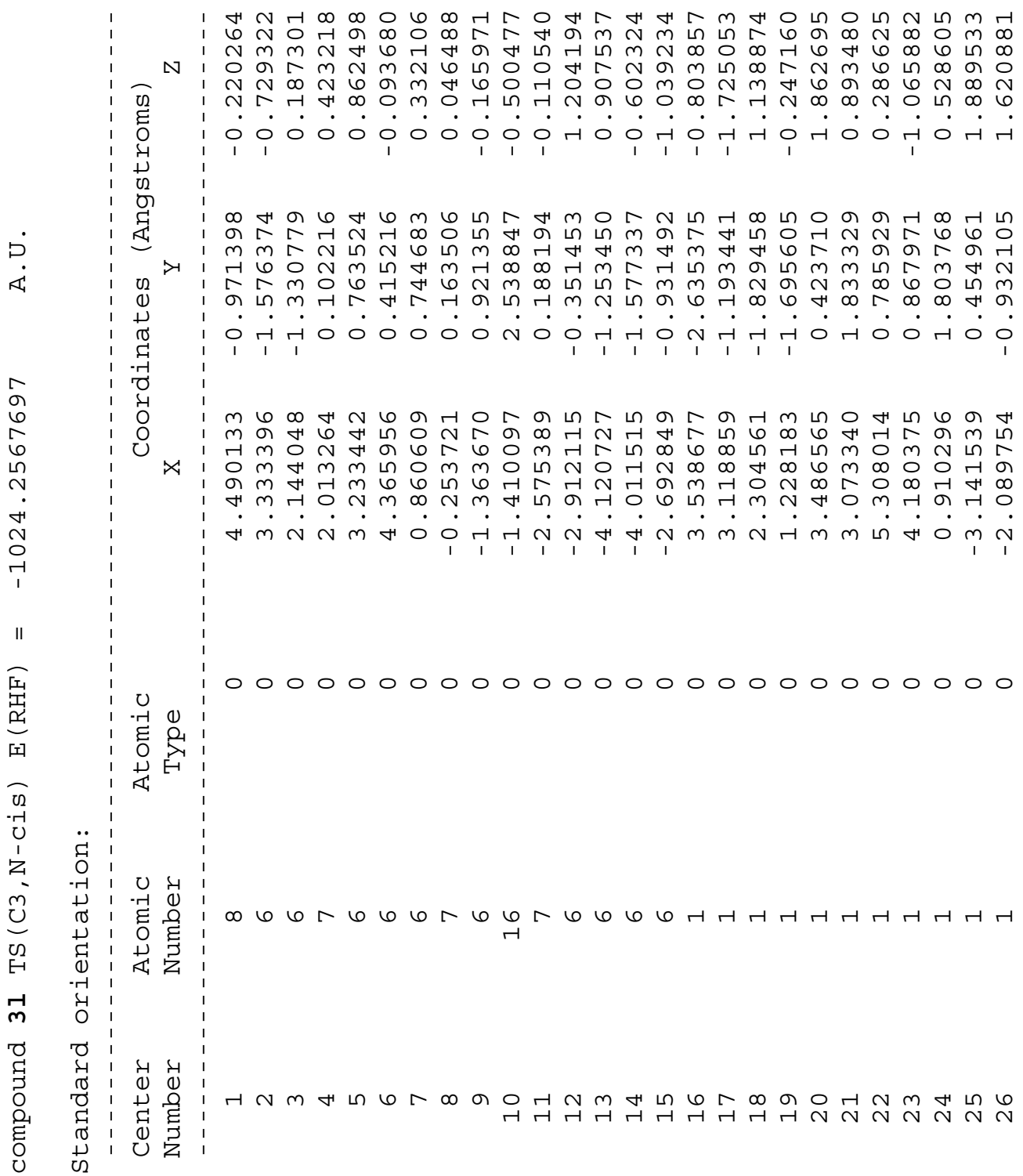




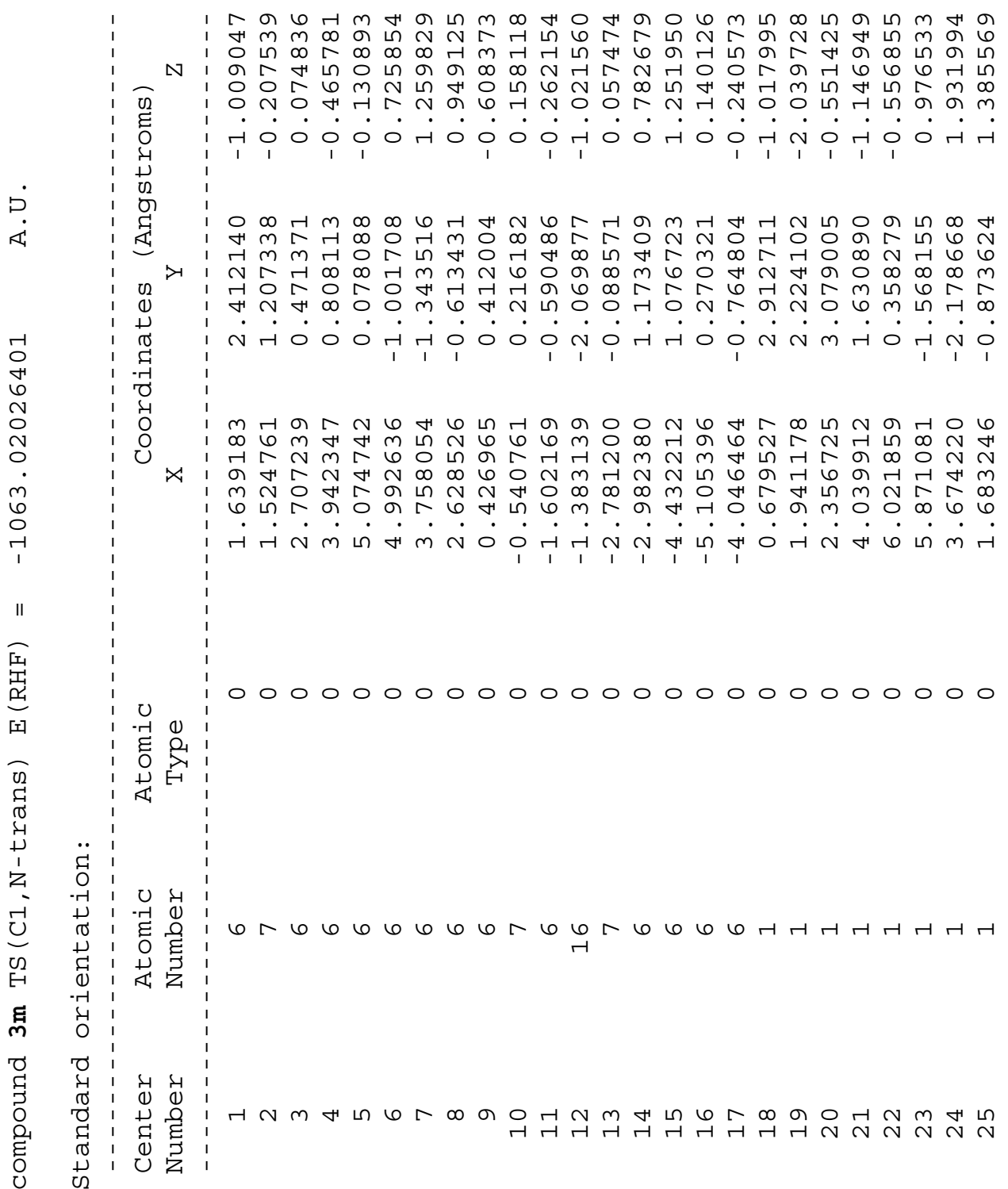




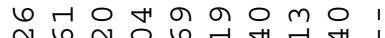
N ก तन 6 म

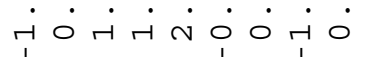

N $\mathrm{m}$ त เ $\infty 6$ เ เ $\circ+N$ H $M N$ H 0 ㄷ 6 เ $m$ नु 000 ,

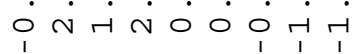

- $\circ \cdots m m$ の $m \infty$ の $6 \mathrm{~m} m$ の 다 하 $m$ ก0 म

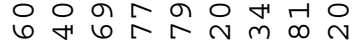

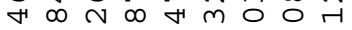

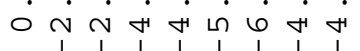

000000000

Н

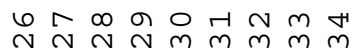

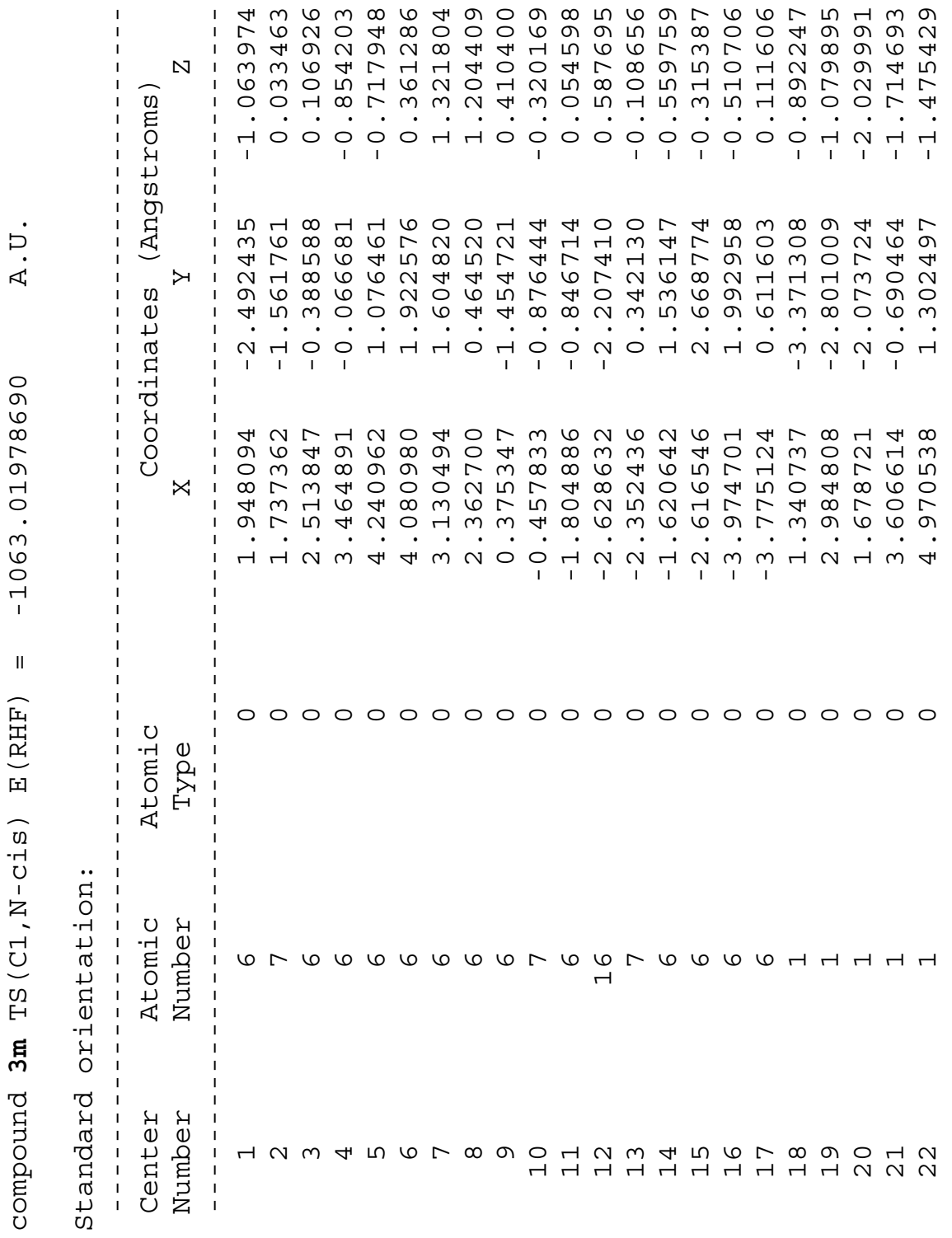

मm $6 \mathrm{~m} \infty 6$ म

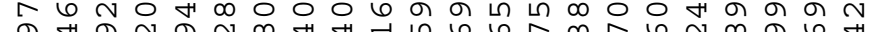
00 - $0 \wedge m m N$ H $m$ m

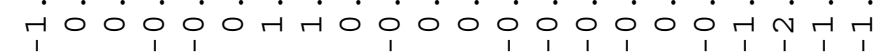

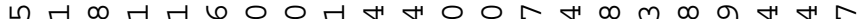

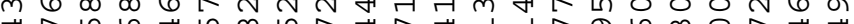

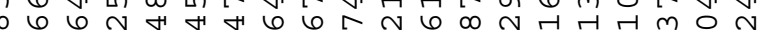
$m \infty 06 \mathrm{~m}$

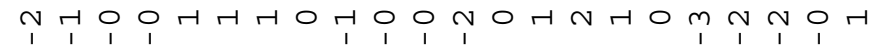
H $N$ h

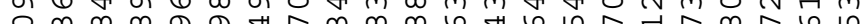

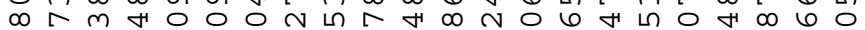

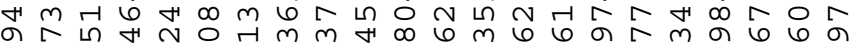

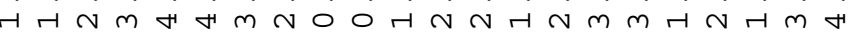

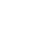


ก म 6 म ก ๓ 0 ก

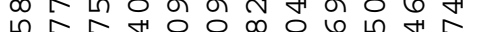

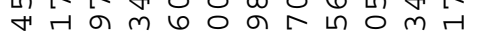

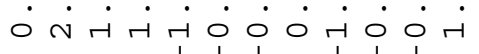

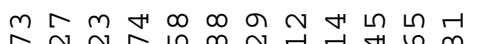

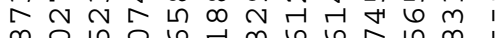

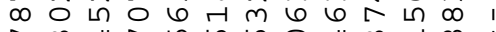
$\hat{0} \mathrm{~m}$ H $\infty$ ก ก म

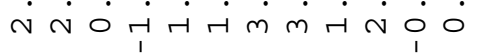

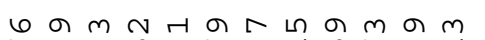

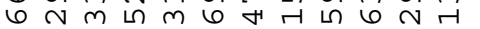
H 6 \& $\infty$ ने त ब ने म

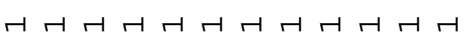

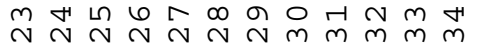
$\begin{array}{lll}1 & 1 \\ 0 & 1 & 1\end{array}$

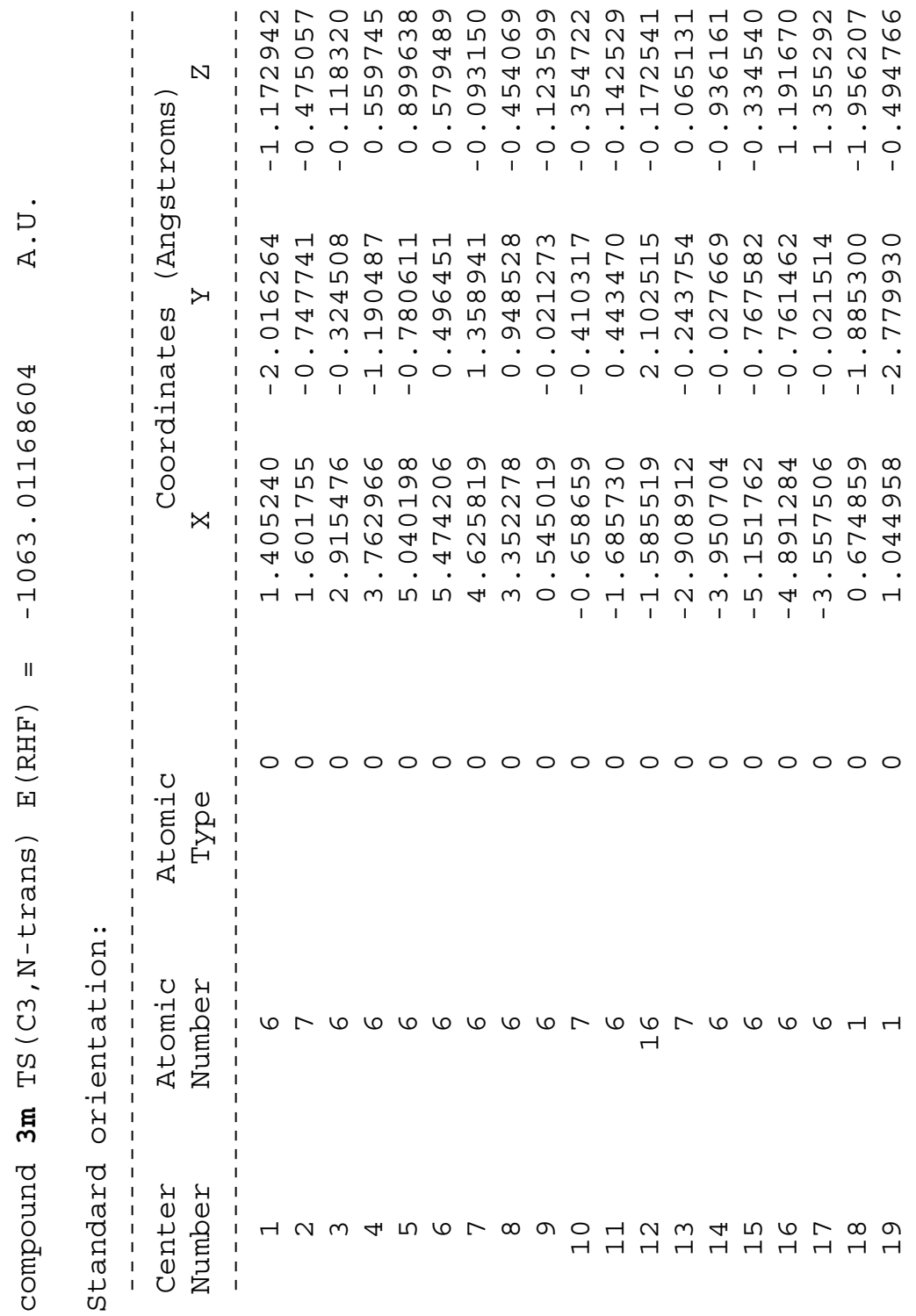

N 0 ए

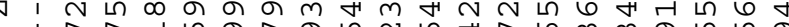
1

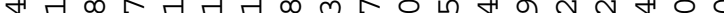

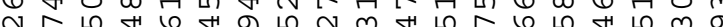
N

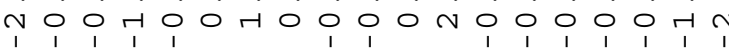

( स $2 \pi 6$

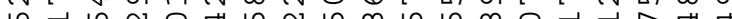

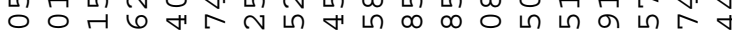

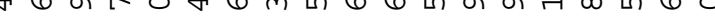
H 
$N$ 대 0 ( $\mathrm{N}$ の

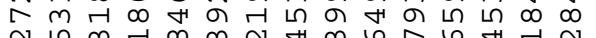

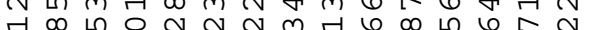

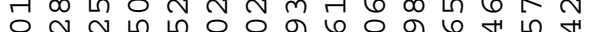

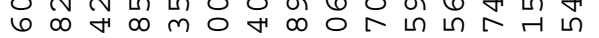

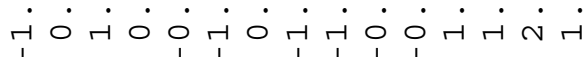

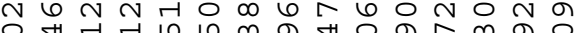
म

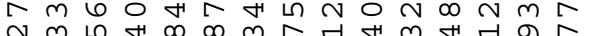
$n m$ n

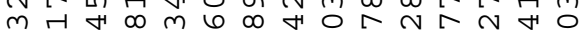

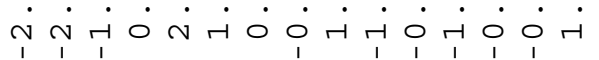

ก

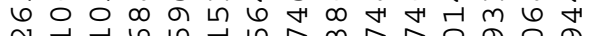

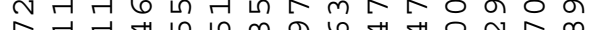
भ त

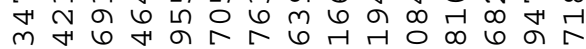

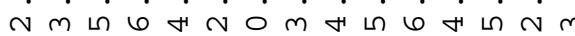
000000000000000

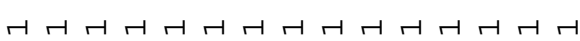

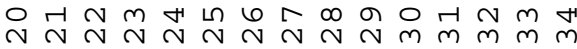

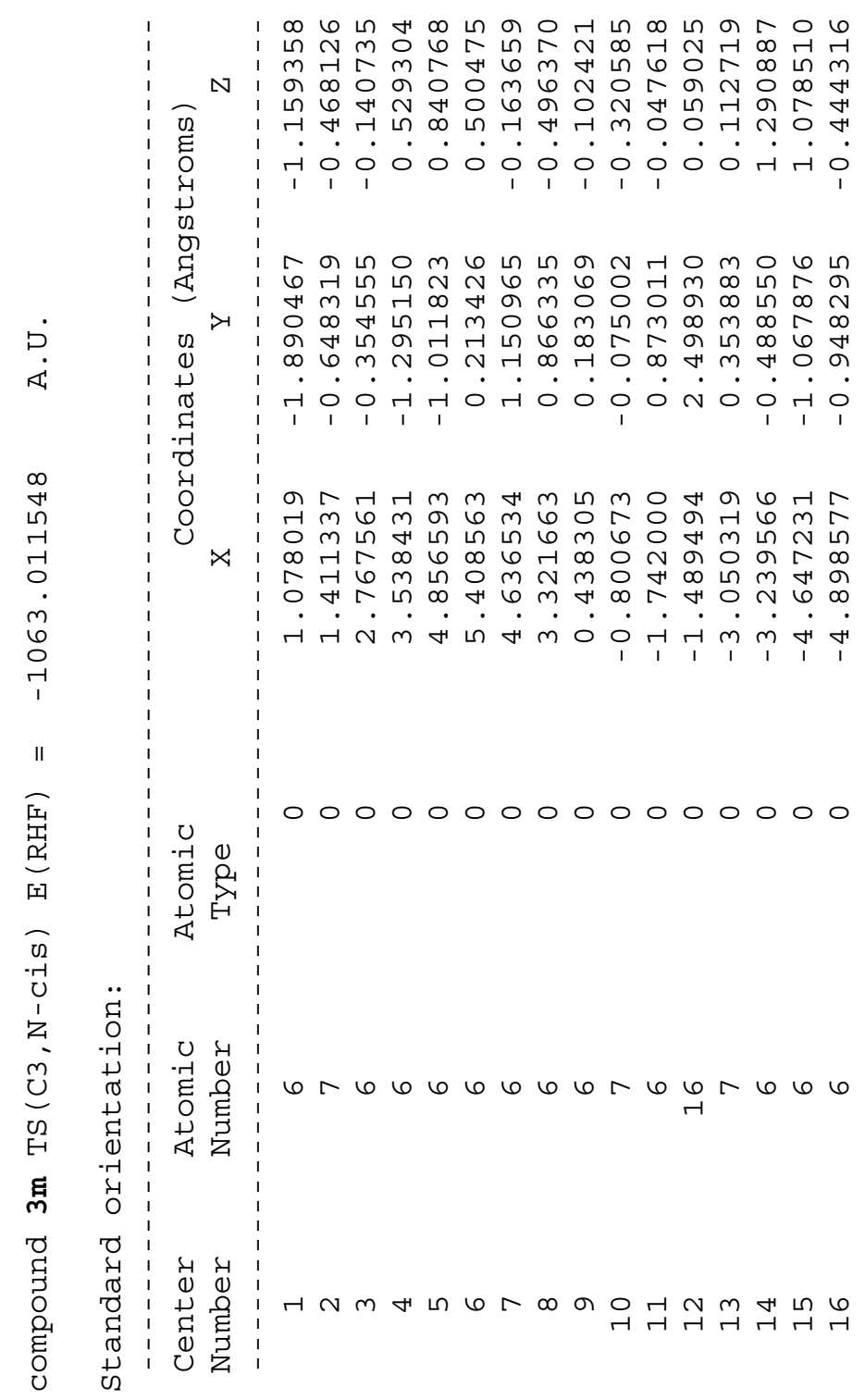




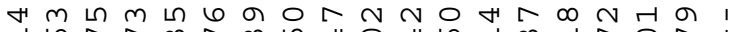

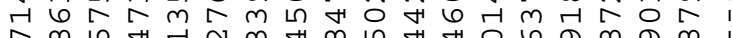

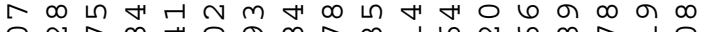

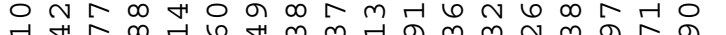
0 ब

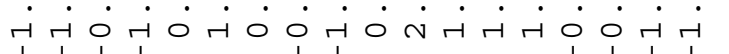

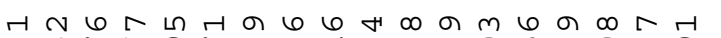

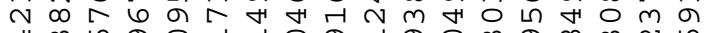

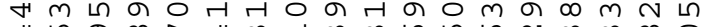

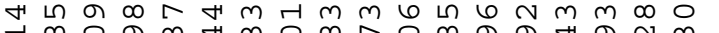

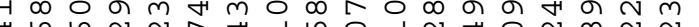
A $6, \pi N N$ N

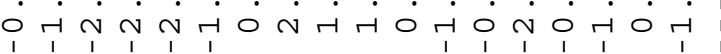

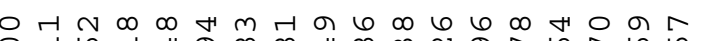
0 त 6 म

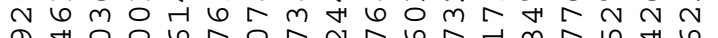
a

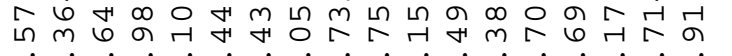

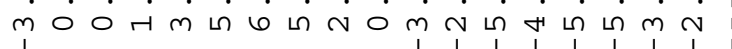

000000000000000000



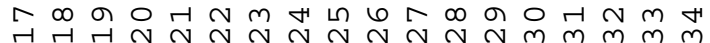

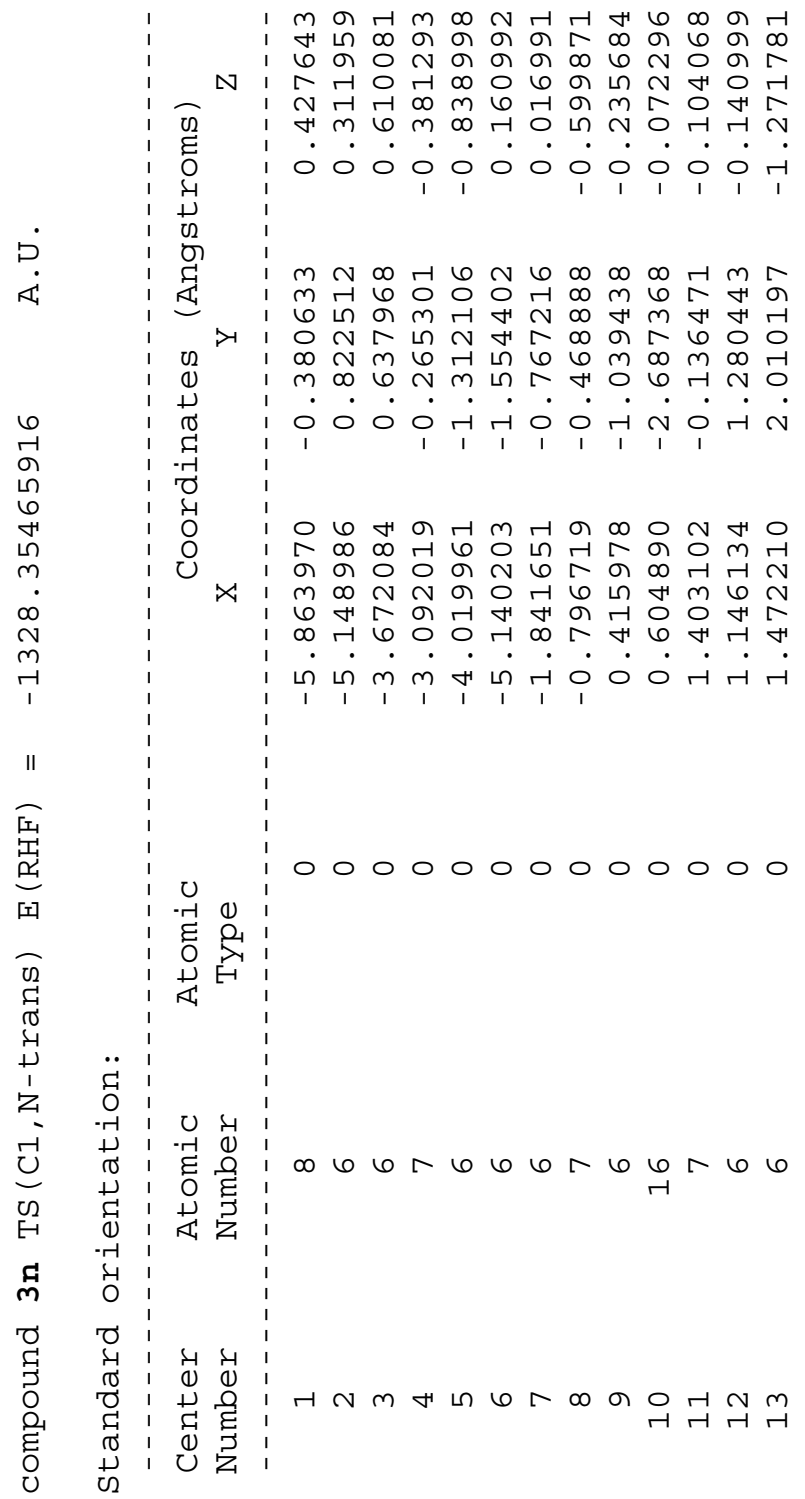


$N$ ட N

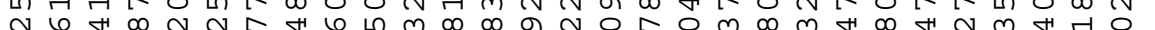
ब

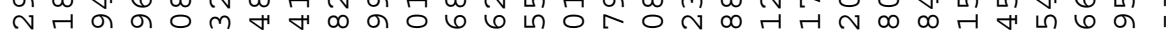

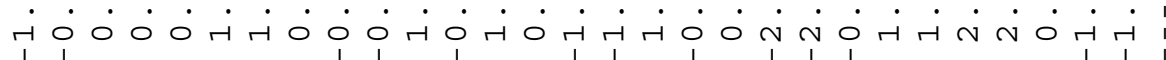

म 4 H

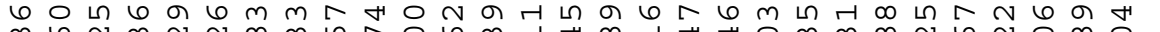

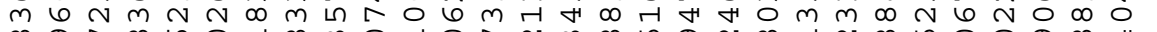

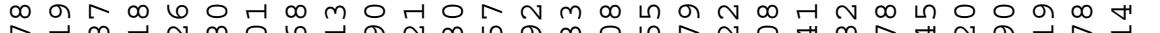
$m$ 구

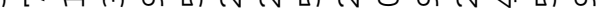

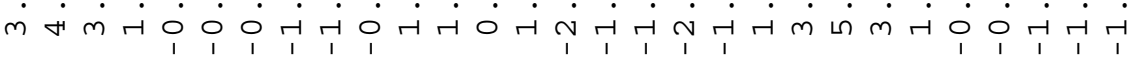

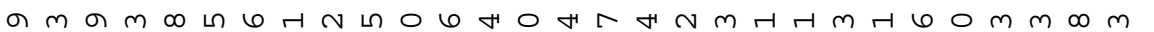

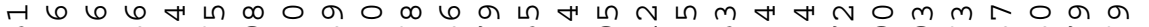

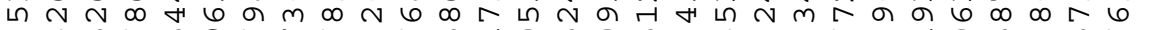

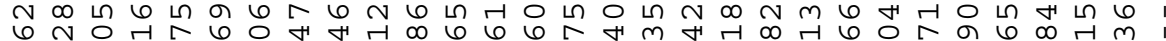

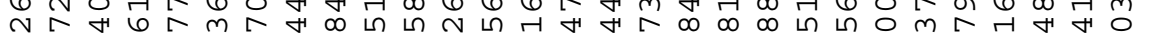

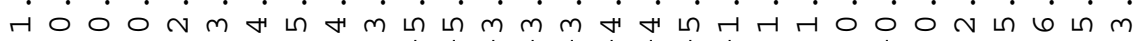
00000000000000000000000000000

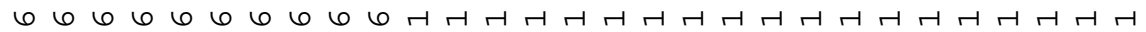

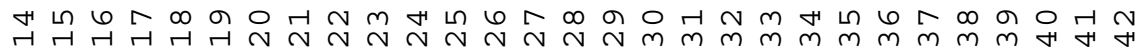

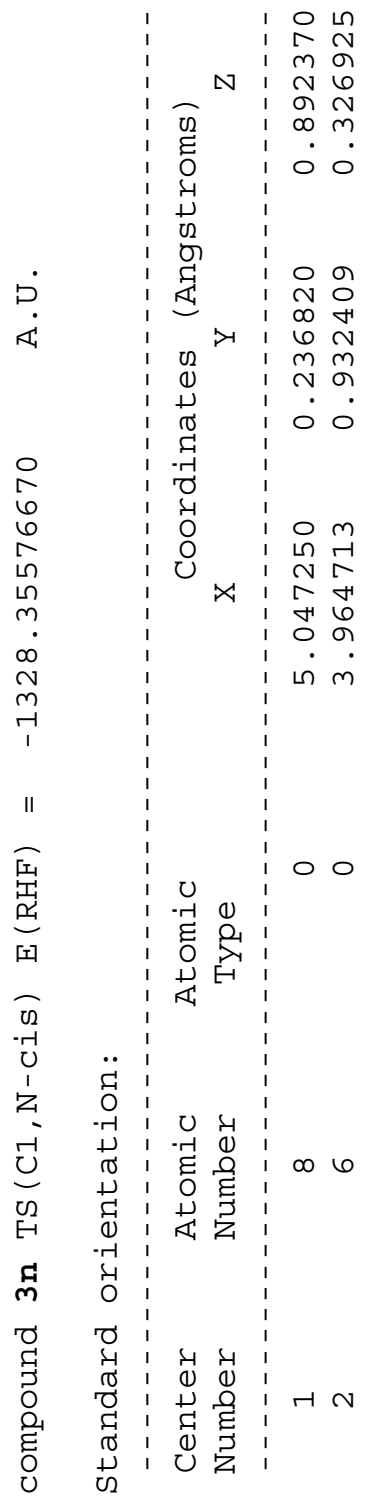


$m \in$ L N

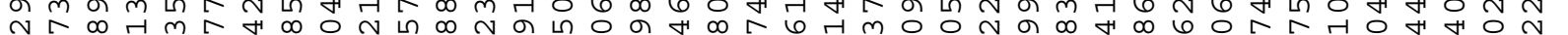

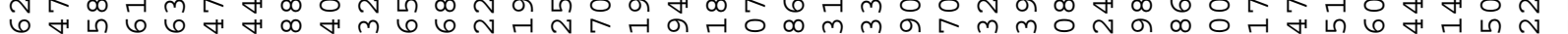
ब $N$ H

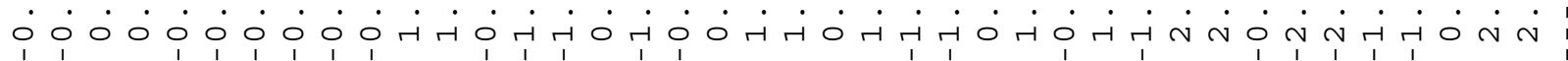

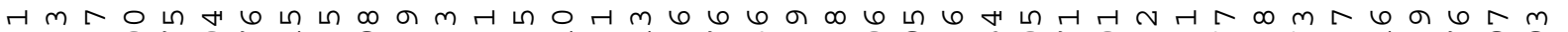

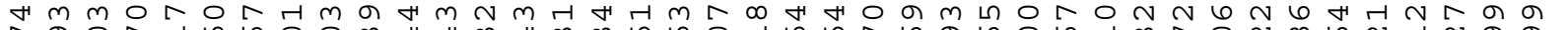

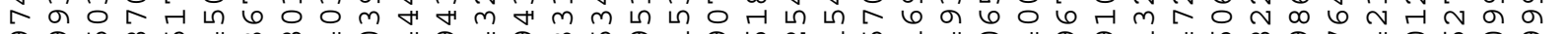

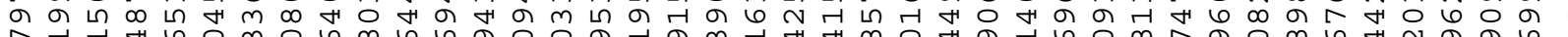

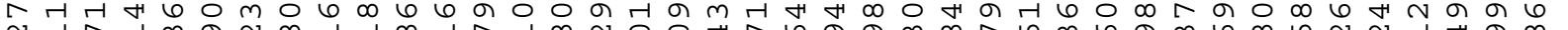
N 0

6 H L F హ゙ भ $\left.\begin{array}{l}0 \\ 0\end{array}\right)$ m m m 0000000000000000000000000000000000000000 6

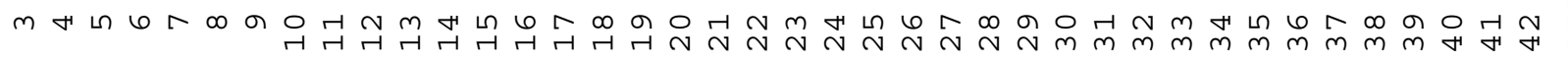




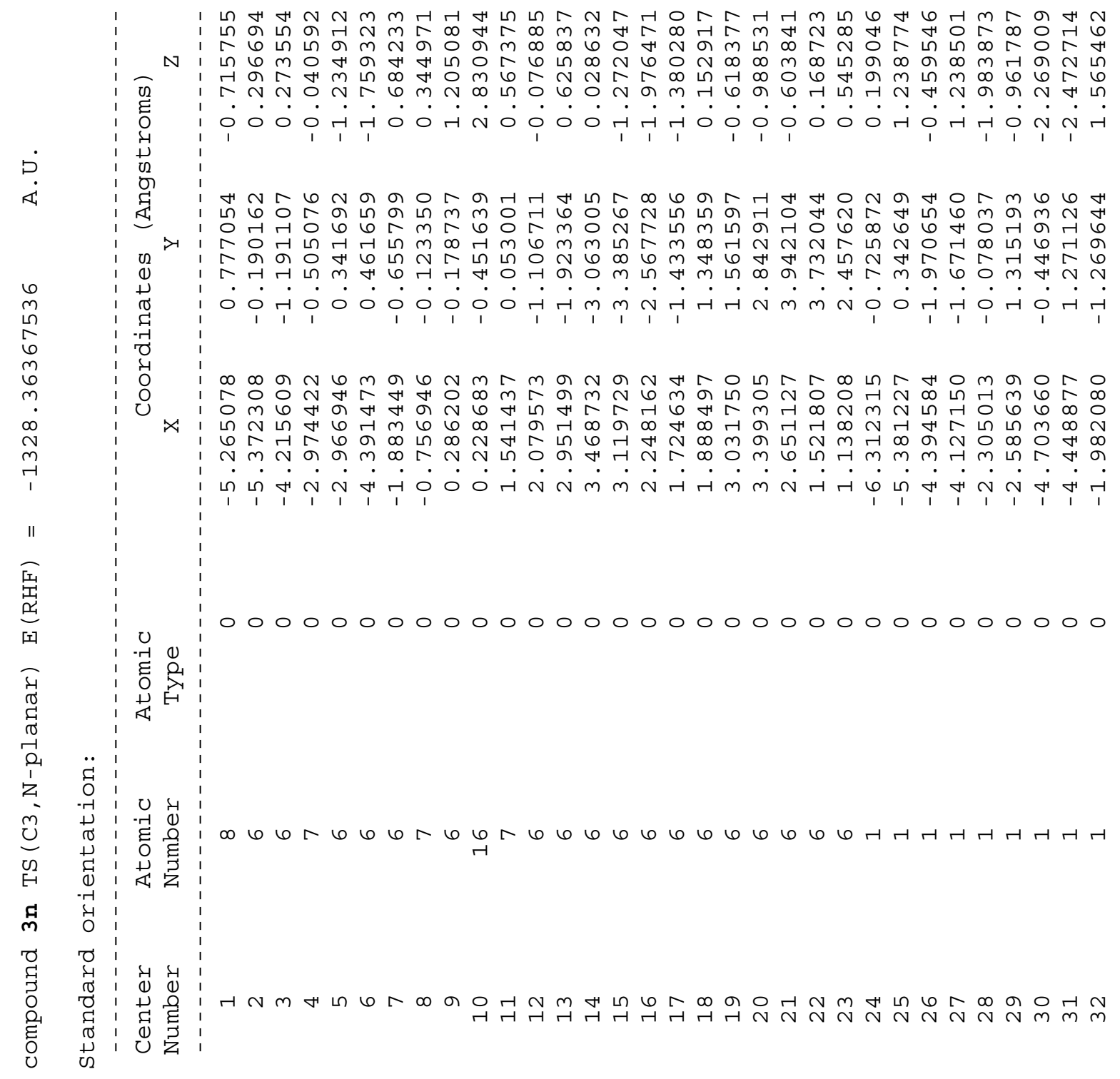


$\infty 6 \circ m$ の $\infty \infty \infty 60$ 드 $\sim N m \in N \sim$

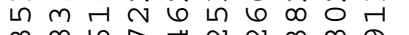

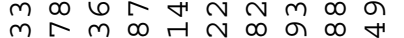
6 in $\sigma$ ब $\dot{0} \dot{0} \dot{0} \dot{0} \dot{0} \dot{0} \dot{0}$

几 $\infty \infty \sim$ ब

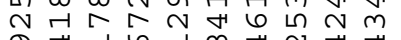
ब म

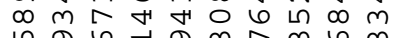

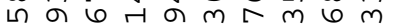
$66 \sim \infty$ ก मि

の

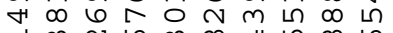

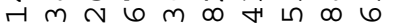
H त $\dot{m} \dot{m} \dot{r} \dot{r} \dot{m} \dot{\sim} \dot{0}$ 0000000000 4444444444

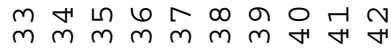

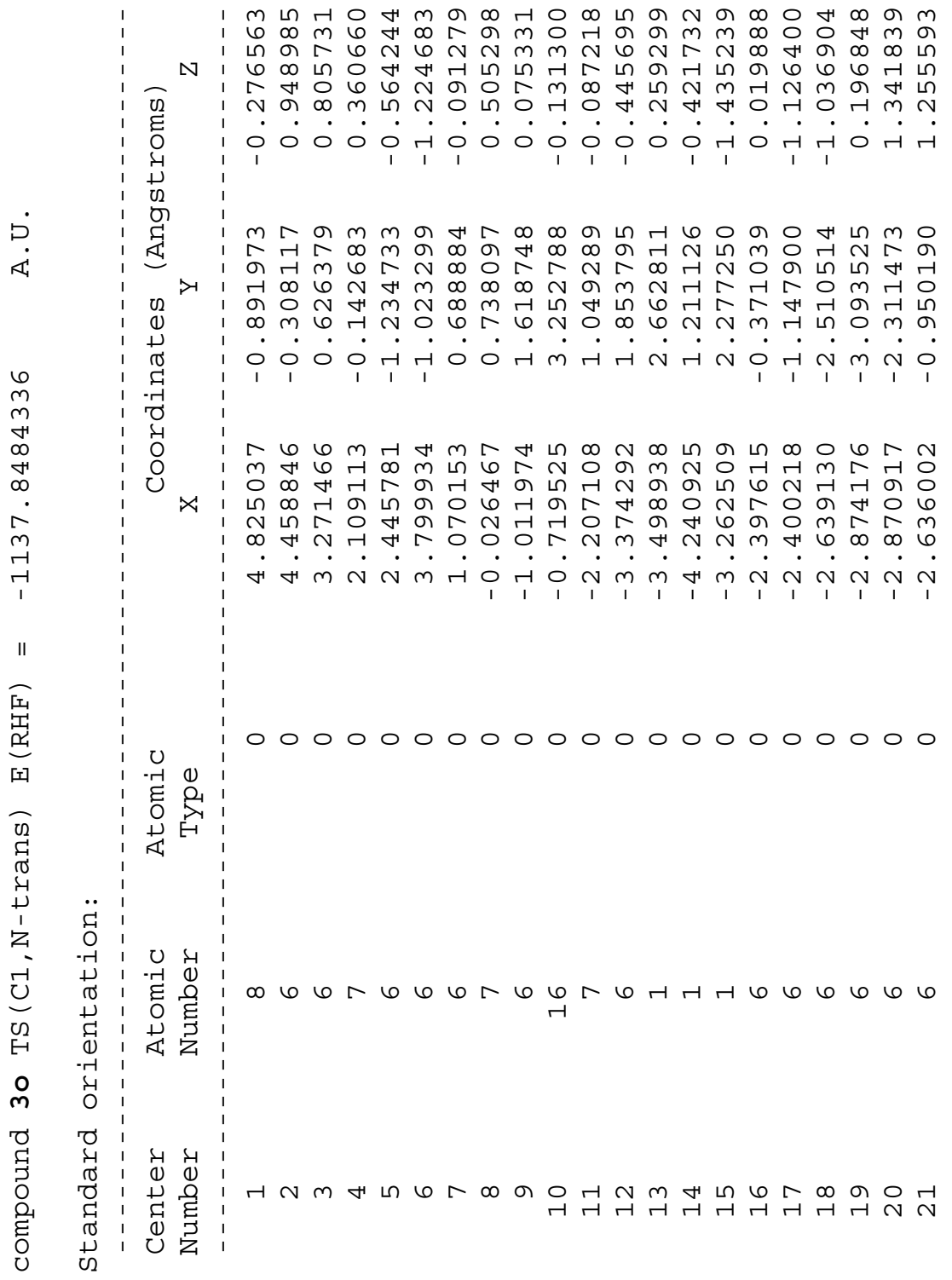


H $N \quad m \infty$ L $N m$ I

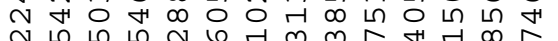
$N$ ก

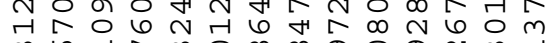

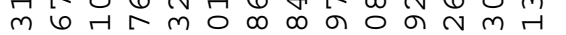

$\therefore$ 小

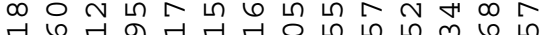
$\pi$ म

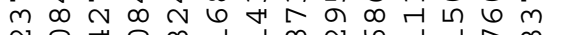

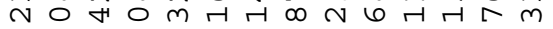
0

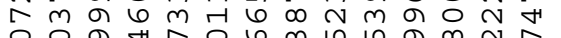

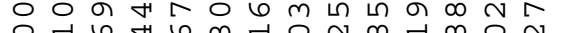

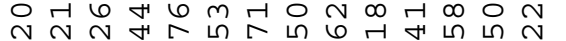
$m \sim$ ก 0 G $\dot{\sim} \dot{\dot{m}} \dot{m} \dot{\sim} \dot{\sim} \dot{m} \dot{H} \dot{N} \dot{\sim} \dot{m} \dot{N}$

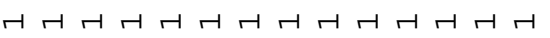

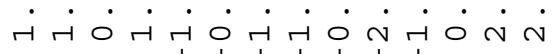

N

00000000000000

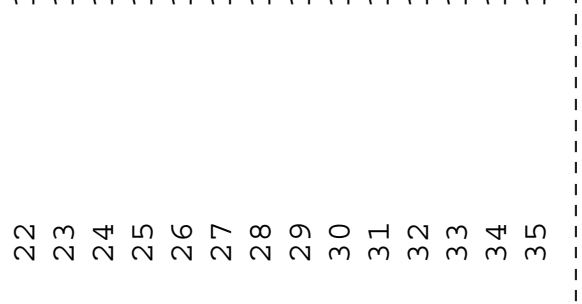

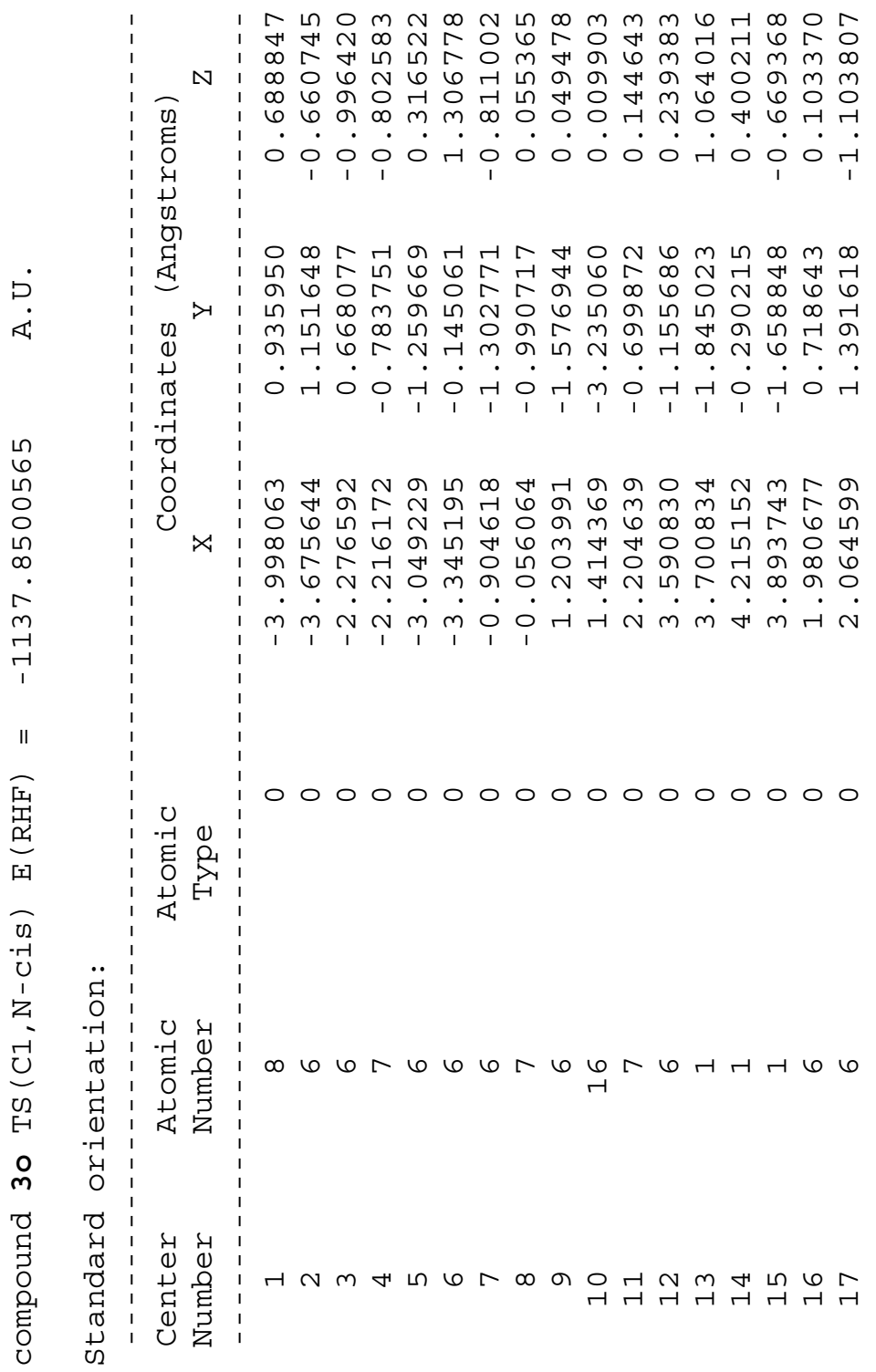

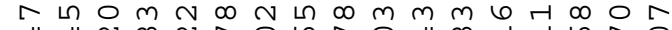

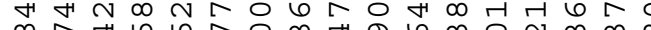

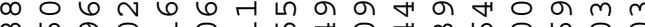

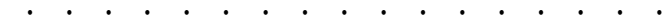

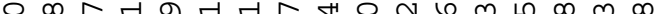
0
0

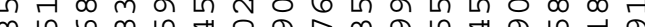
$\dot{0} \dot{0} \dot{0} \dot{0} \dot{0} \dot{0} \dot{0} \dot{0} \dot{0} \dot{0} \dot{0}$

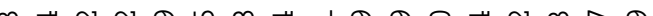
60

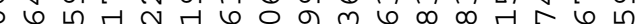

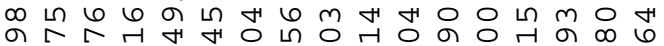

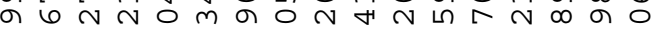
$m m N N m m 00 H H N m m+m H N$




○ $\circ \mathrm{mm}$.

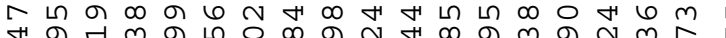

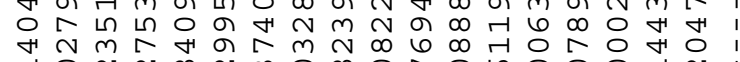

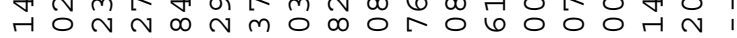

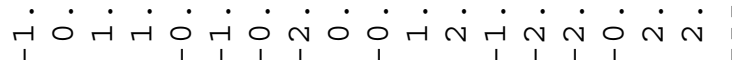

I

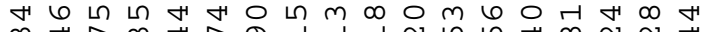

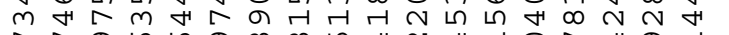

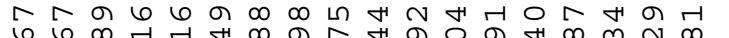

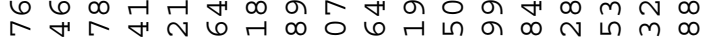

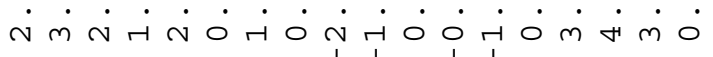

เ 6 の

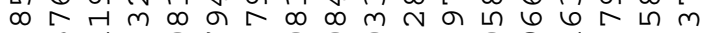
$m$ m

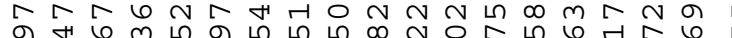
の

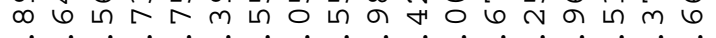

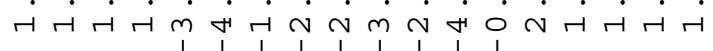

000000000000000000

66 6

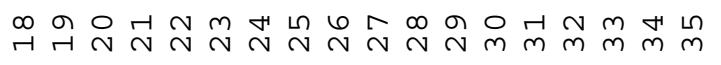

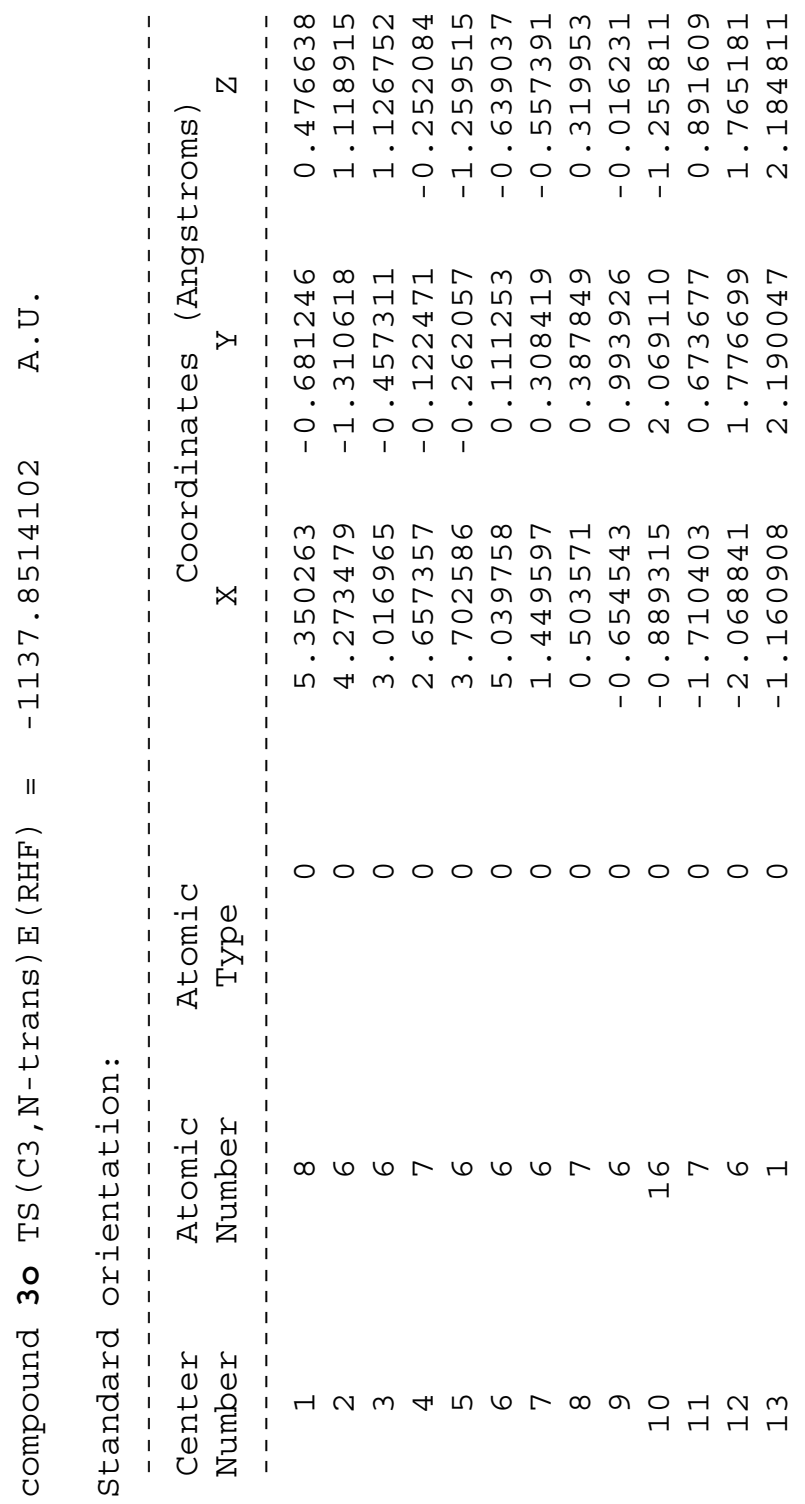


$\Lambda \infty N$ の

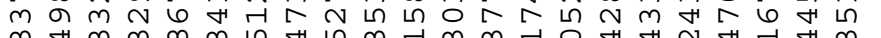

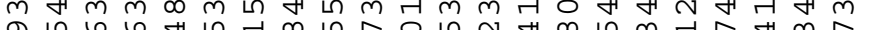

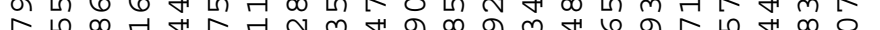

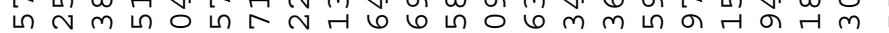

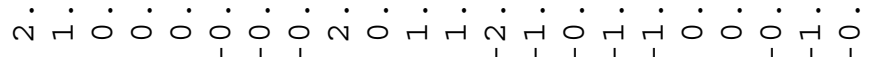

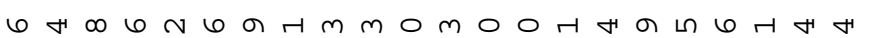

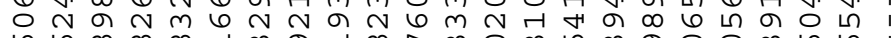

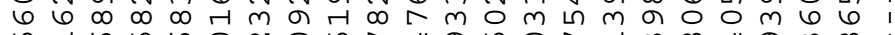
ก ब $\mathrm{n}$ เ $m$ म

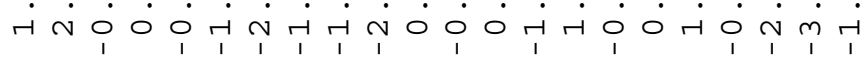

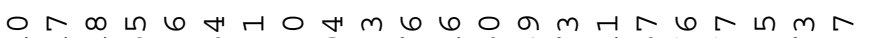

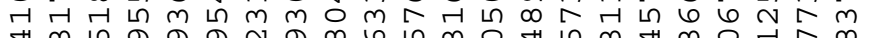

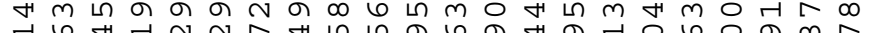
당

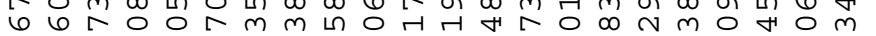

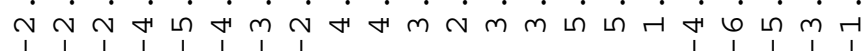

0000000000000000000000

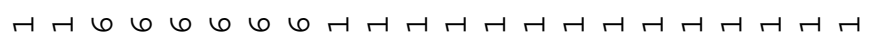

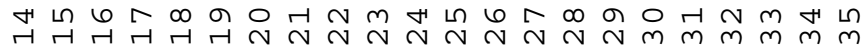

L in 100 in 1 เ $\mathrm{N}: \mathrm{r}$ 月 (n) m है (1) U ర్త 岩 $\rightarrow$ Un $>>1$ (1)

$1 \underset{1}{1}$

न

i

10

U

$\times 1$

a $\infty 6 \infty m m a r$ ก $N$ ब ถึ 人。 $6 \wedge m$ เ $\infty 6$ म $N$ $\dot{r} \dot{m} \dot{\sim} \dot{m} \dot{0} 0$

000000000

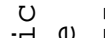
है 质 $\ddot{c}$ 0 $\begin{array}{llll}-4 & 1 & 0 & 0 \\ -4 & 1\end{array}$

\%

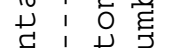
(1) 茛安 萜

o

(

告 1 मे 4 O : 
$m$ m

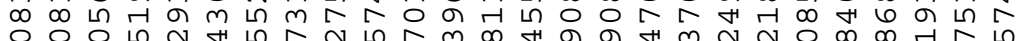

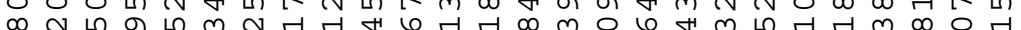

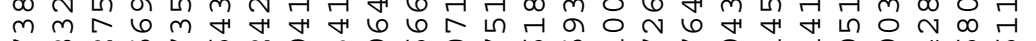

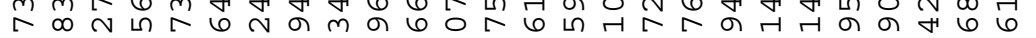

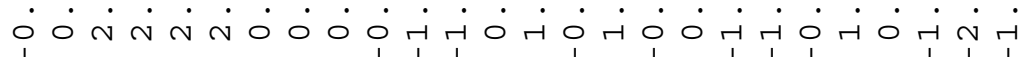

$\infty \wedge N \wedge \infty N 60$ เ

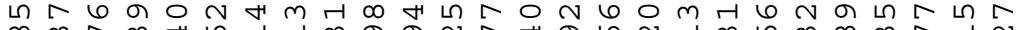

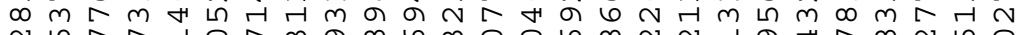
$N$ I m $\infty \circ 0$ の $\infty$ N $\mathrm{m} N 00$ の

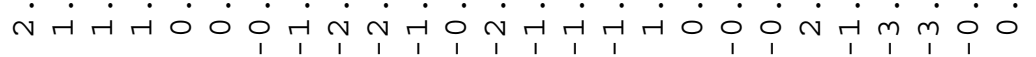

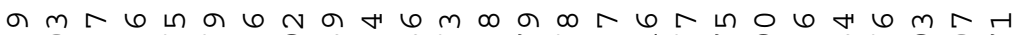

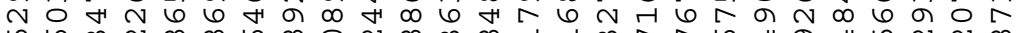

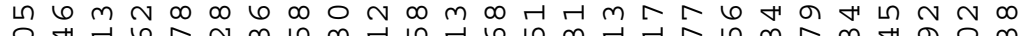
하

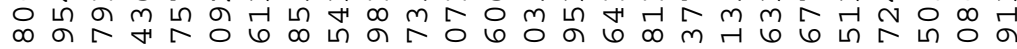

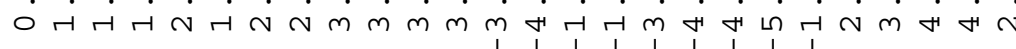
00000000000000000000000000 6

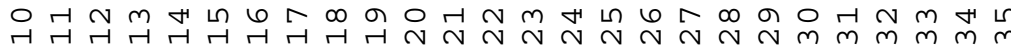

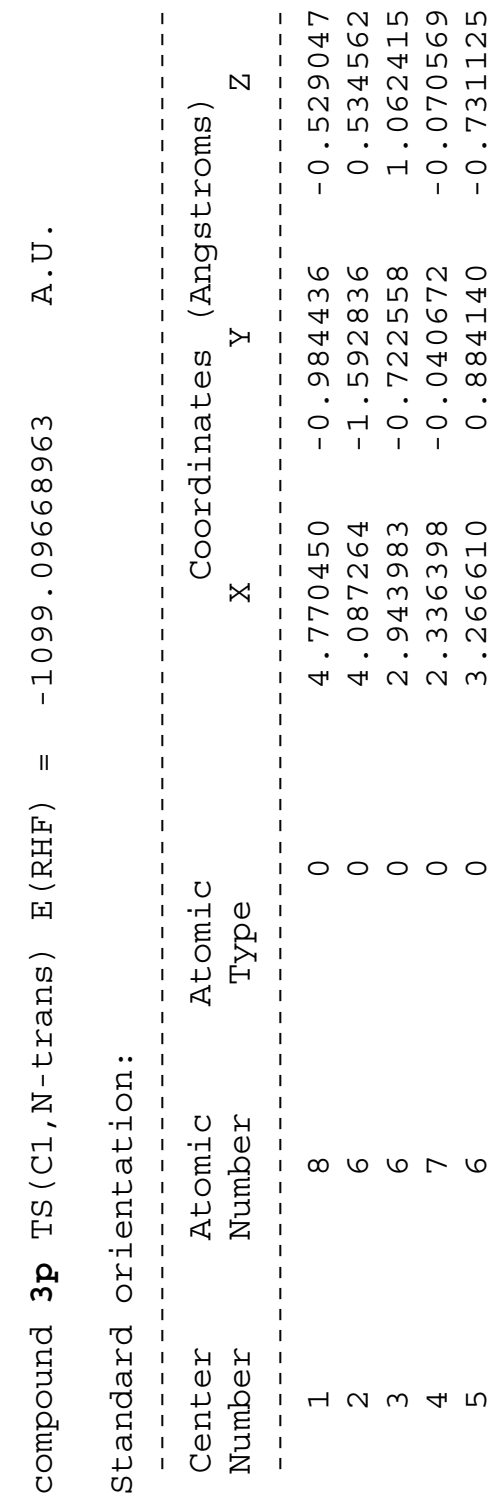




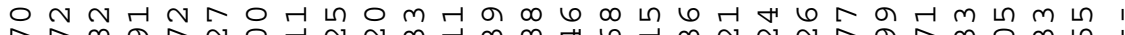
I $\infty$ ब $N$ N $m 6 m \Gamma N N$ H

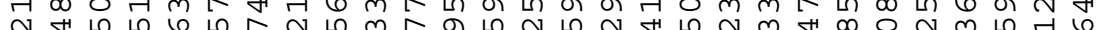
ก $N$ O

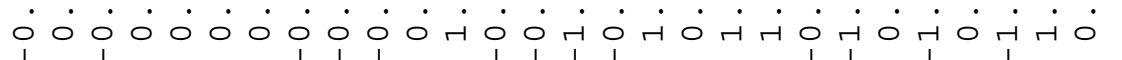

$m \infty m \sim$ H

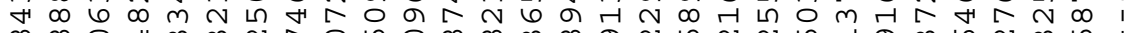

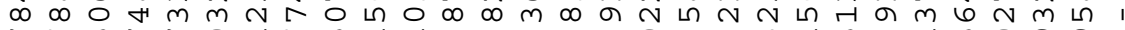

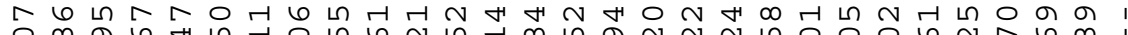

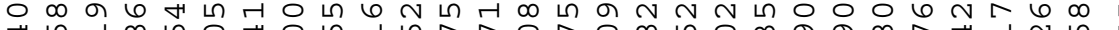
स

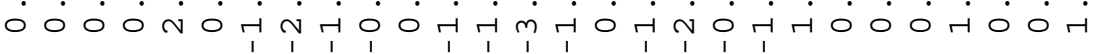

- $m$ a

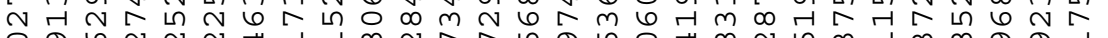

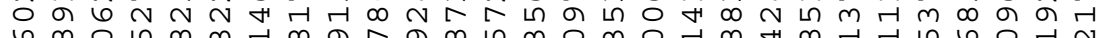

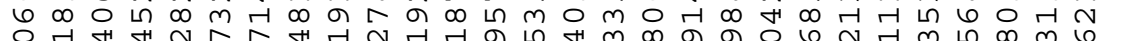

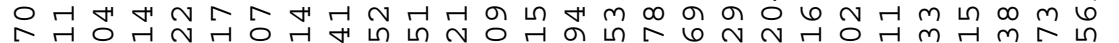
-

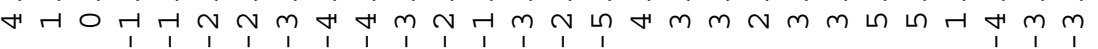
0000000000000000000000000000

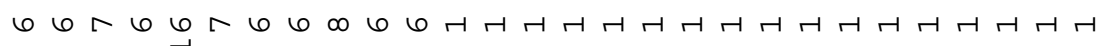
6 $\infty$ の

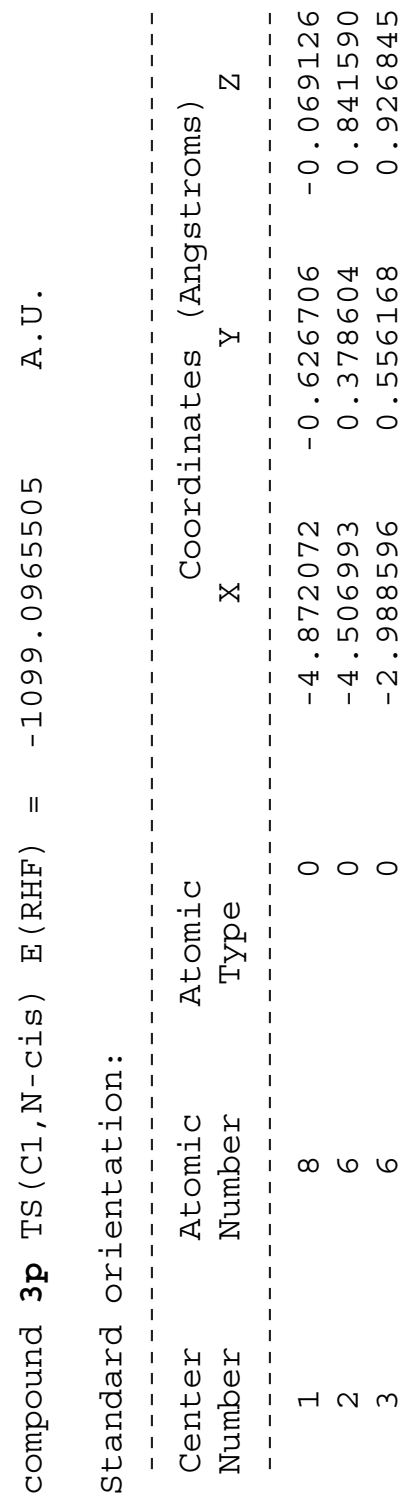


เก $N$ H

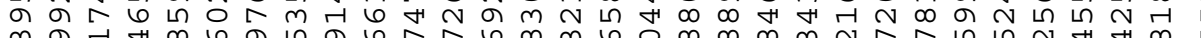
$m \sim 40$

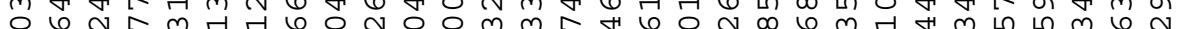

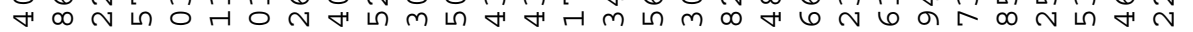

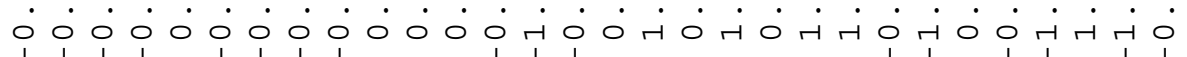

A

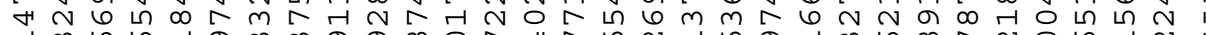

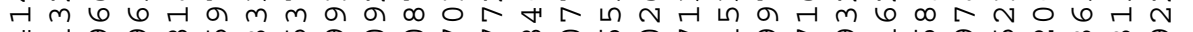

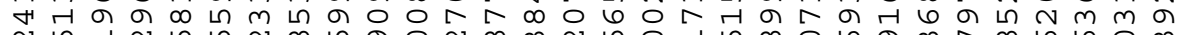

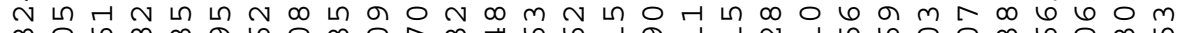

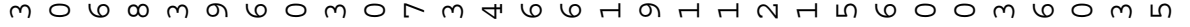

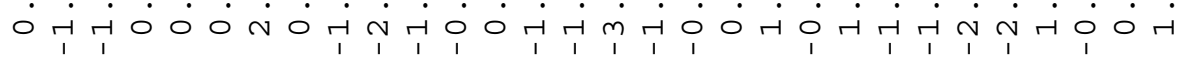

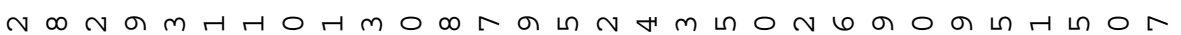

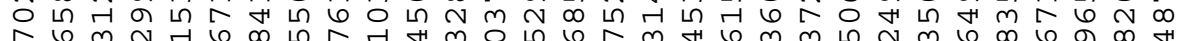

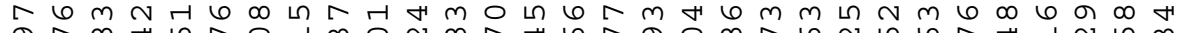
ติ अ 6 बनत $\mathrm{r}$ त

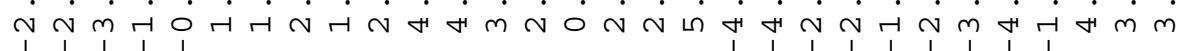


G 0 h

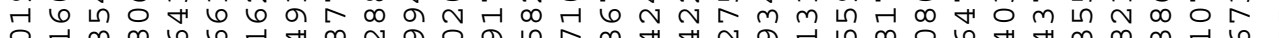

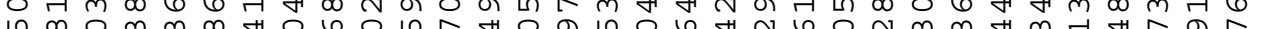

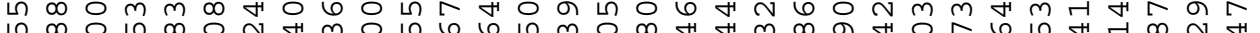

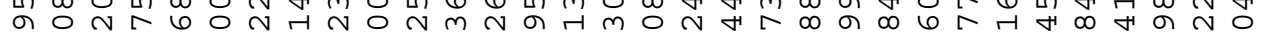

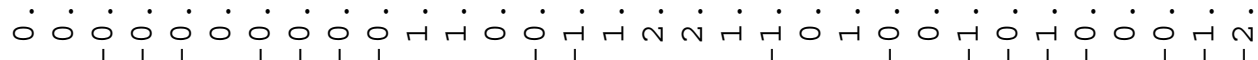

の $\infty$ H $0 \wedge \circ$ H 606 H०

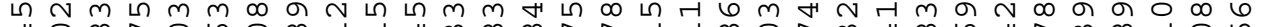

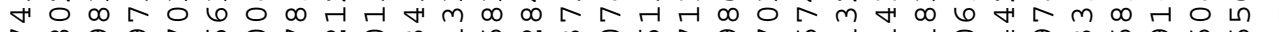

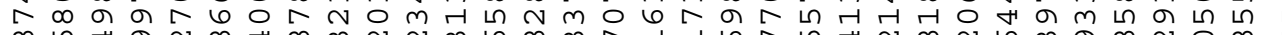

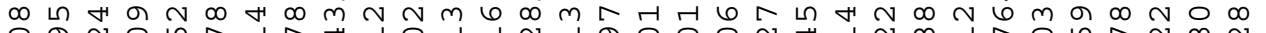

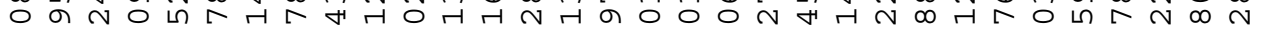

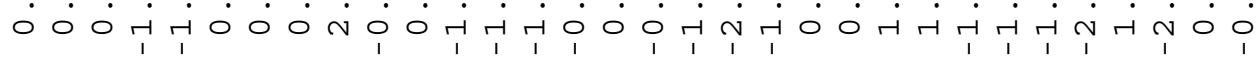

$\circ \circ$ เ

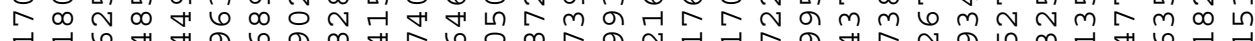
답 v

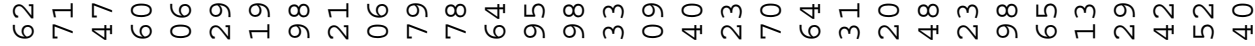

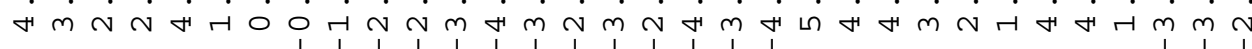




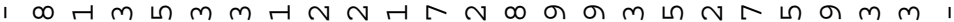

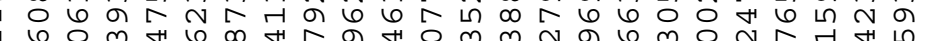
$N$, (1) बतरत 6 ॠ E

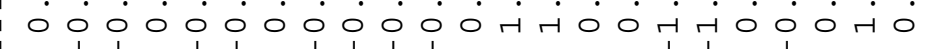

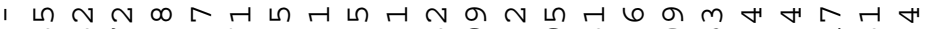

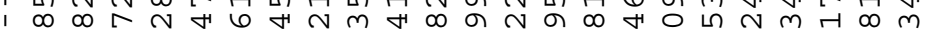
i $m$ H

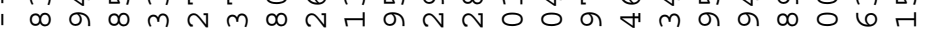

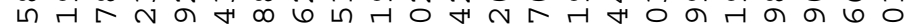

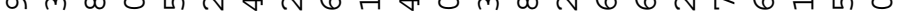

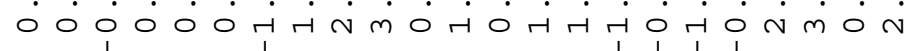

N $\infty$ H

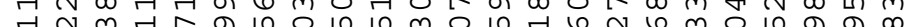
r

$x, 4 r$ in

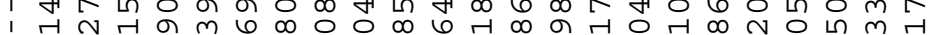
ก $\infty$ म

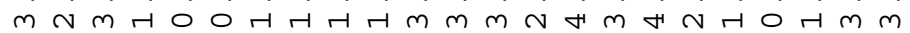

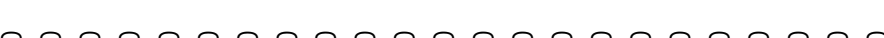

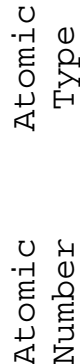
(4)

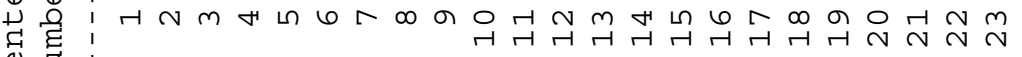

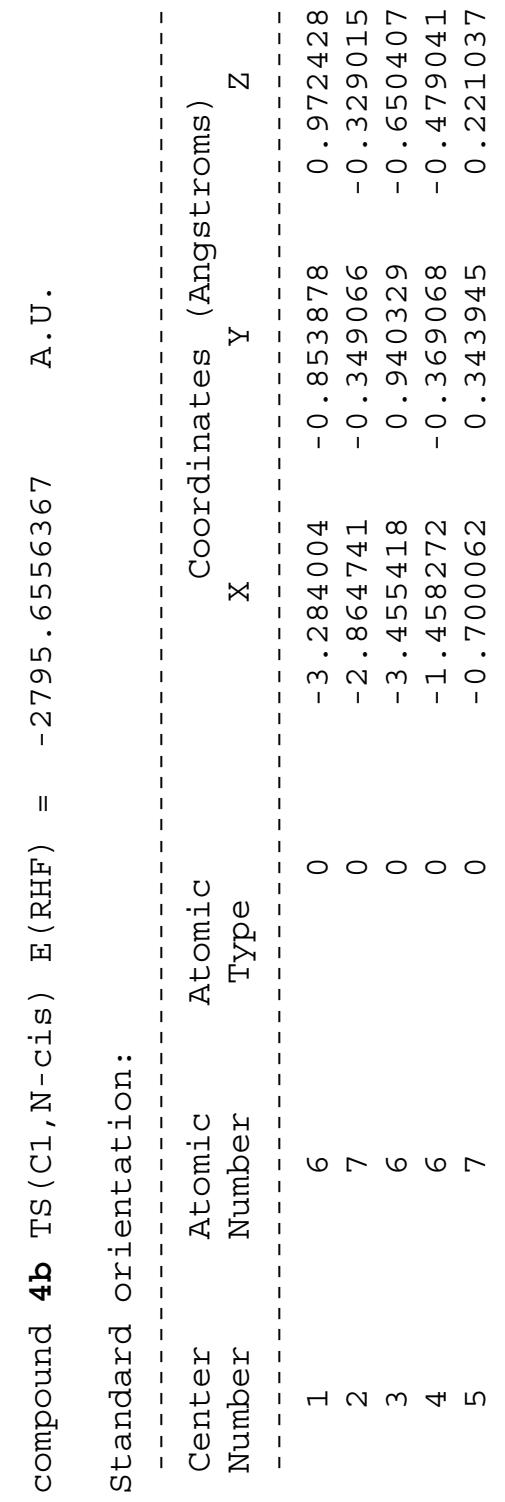


N O N $M N$ L अ

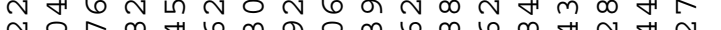

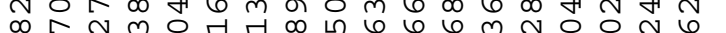

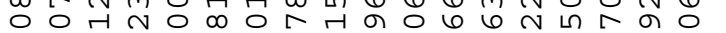

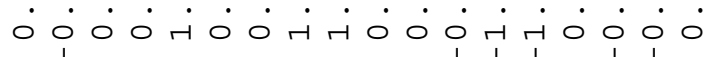

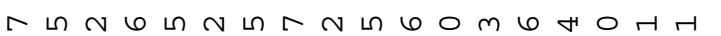

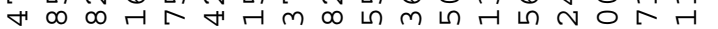
स H

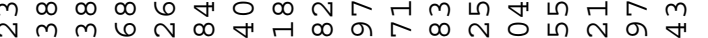

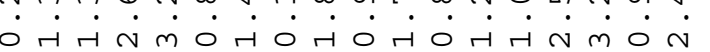

$m+m N$ H

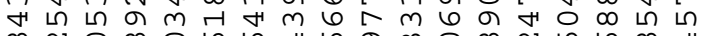

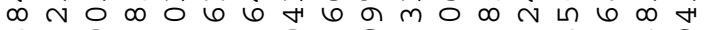

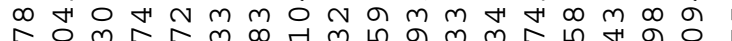

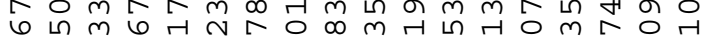

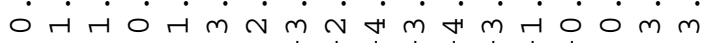
000000000000000000 4

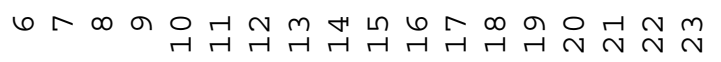

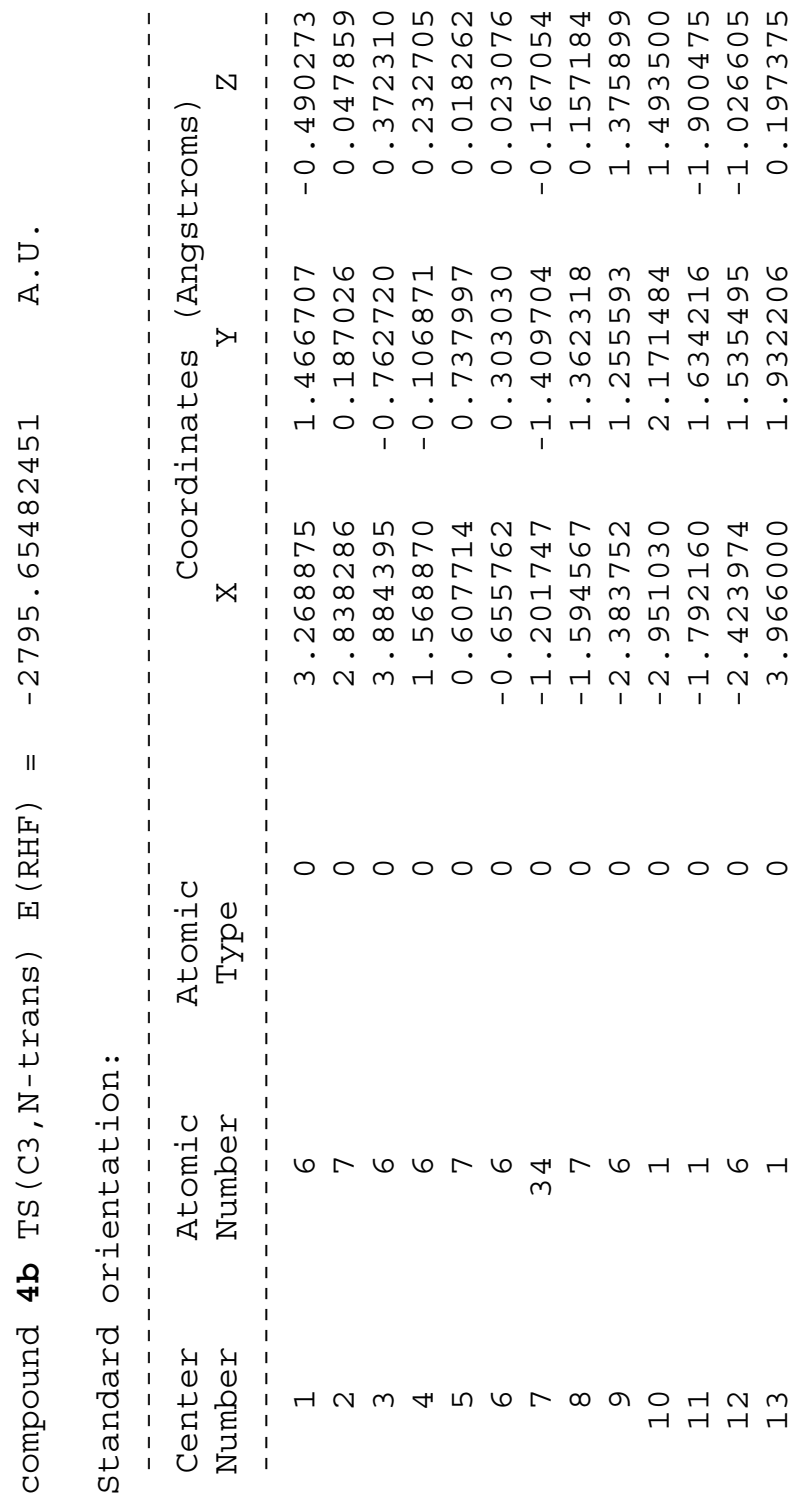


A 4 mo $m$ 대 $\infty$ 네 भ

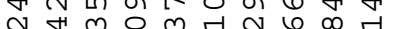
6 म $\dot{\circ} \overrightarrow{1} \dot{0} 0 \dot{0} 0 \dot{0} \vec{r} \dot{0}$

त 가 भ न ते ब

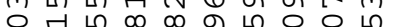
त $m m$ ब 60 म

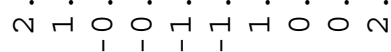

4๐大 $\circ m$ म $N$ क $N$ क $N$ N का मे त क

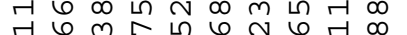
4र म ते $\dot{m} \dot{m} \dot{\vec{r}} \dot{m} \dot{m}$ 0000000000 444444444

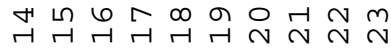

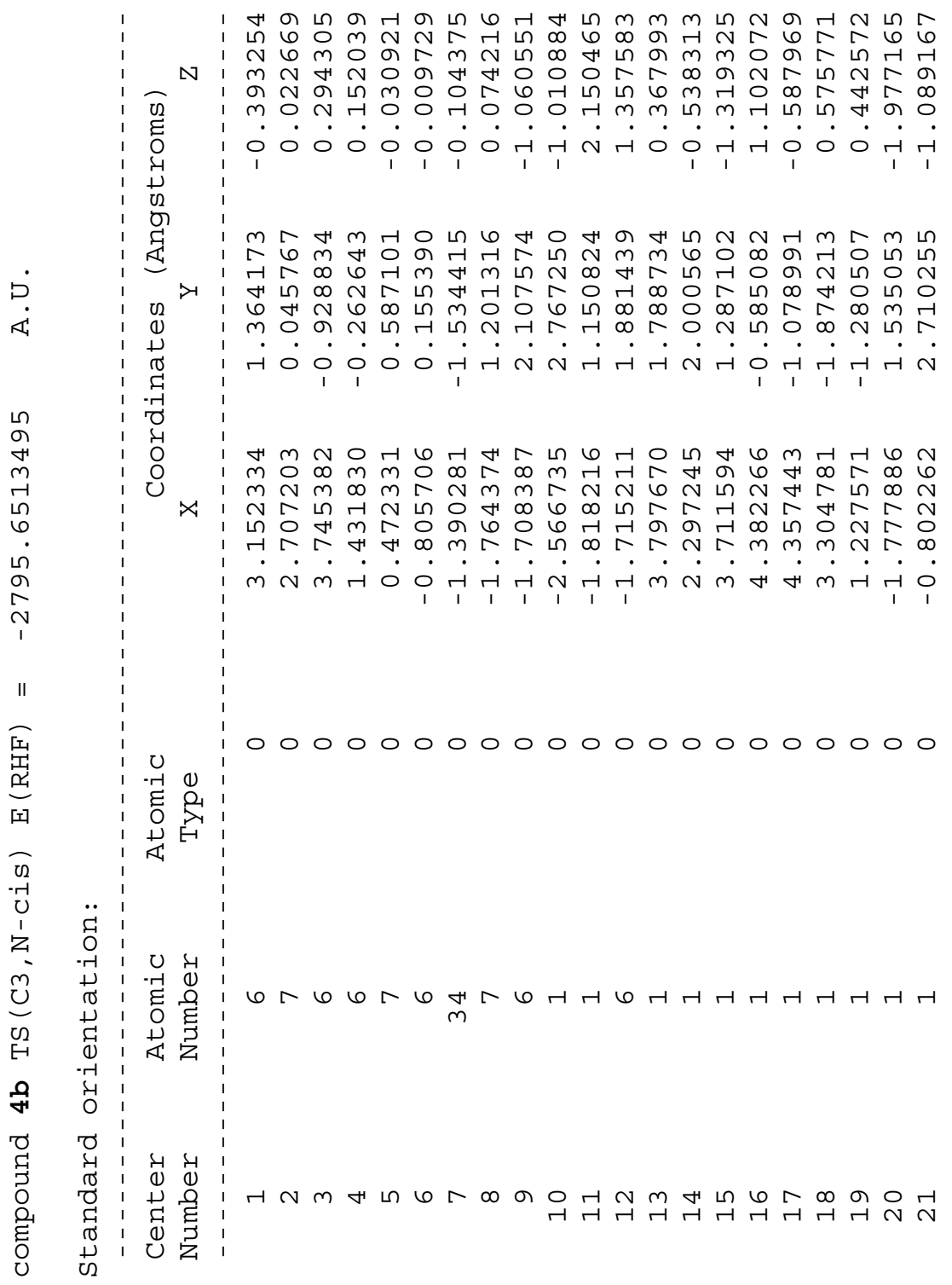




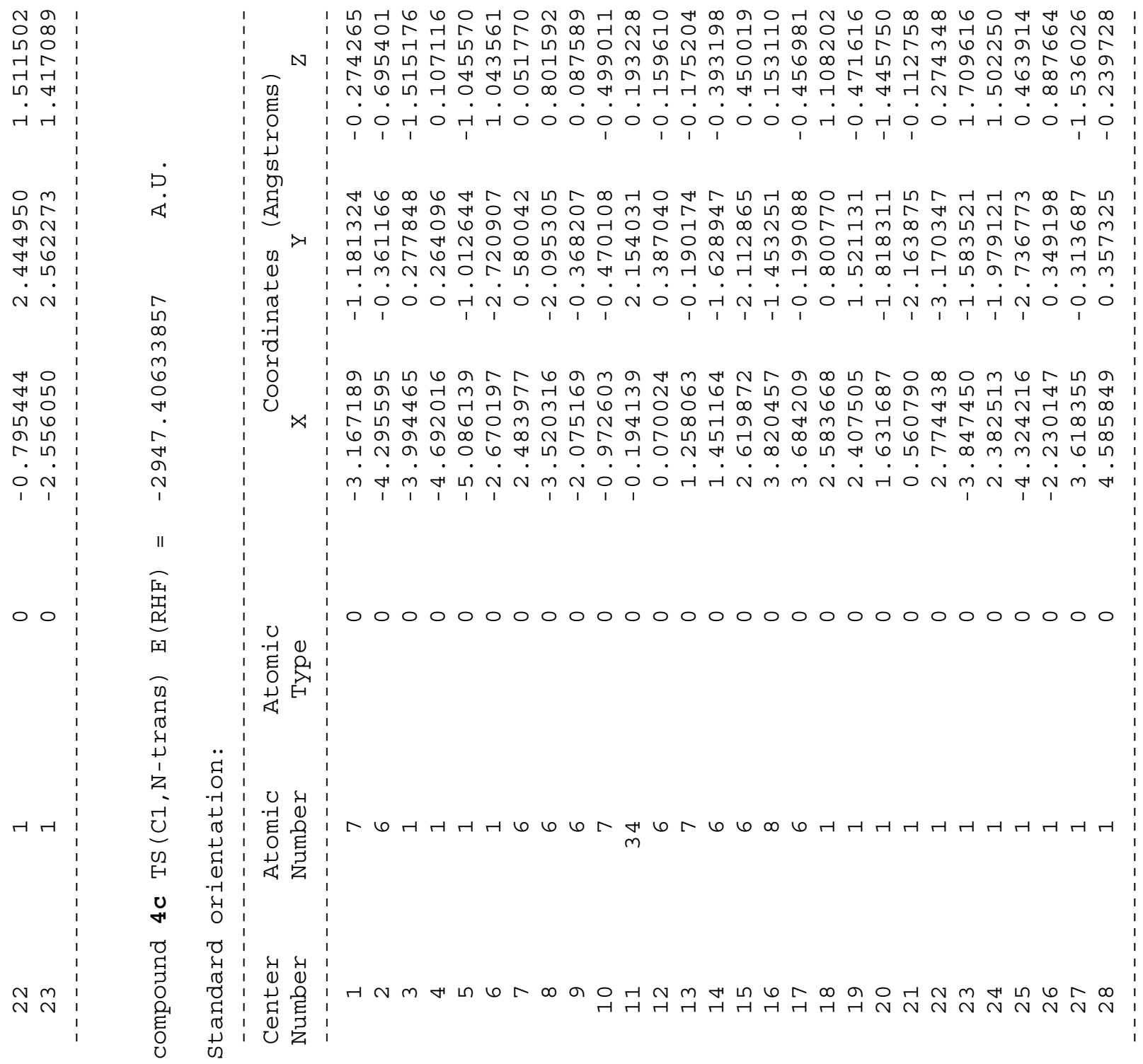




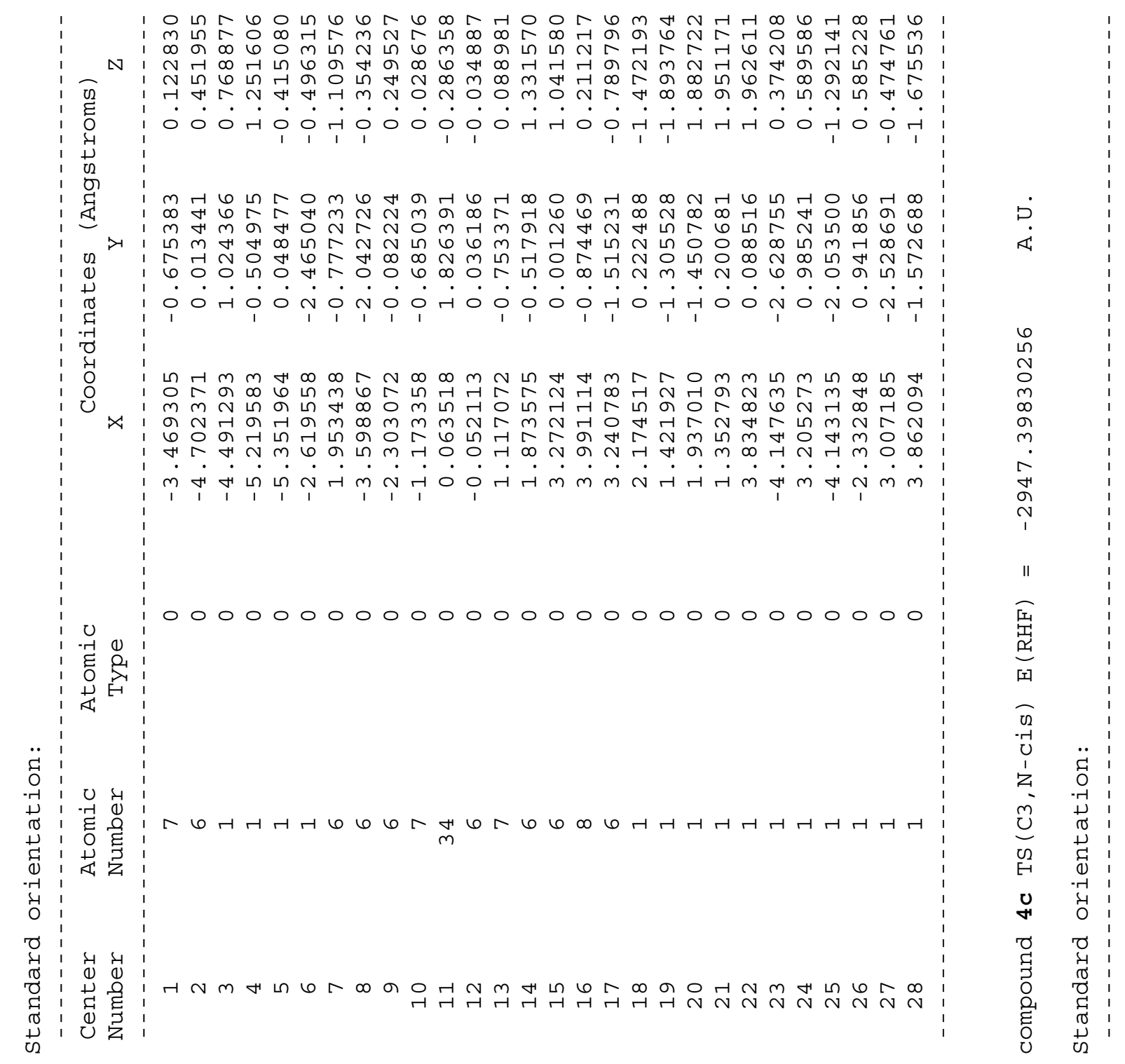


I अ

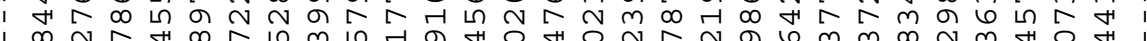

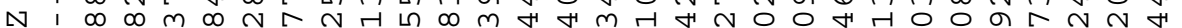
a $n$

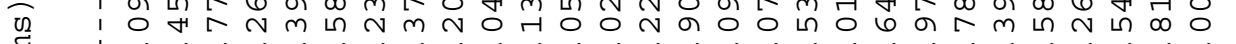

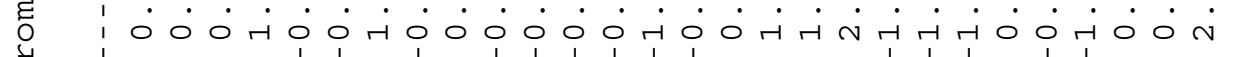

の 0 の

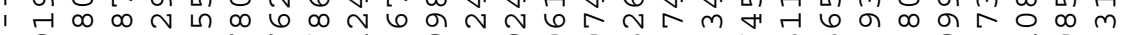

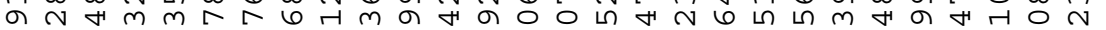

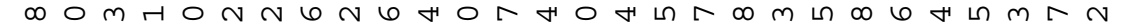

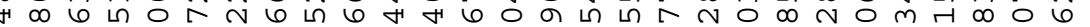

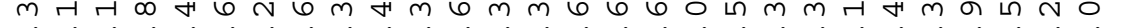

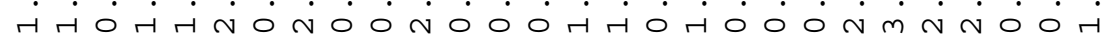

N

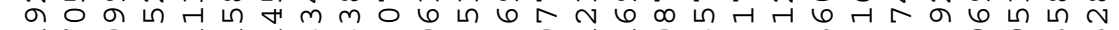
न $N$ m

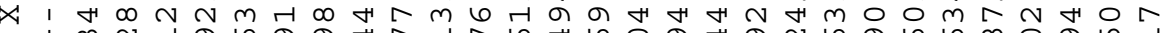
N

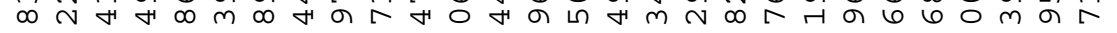
NAHAH 0000000000000000000000000000

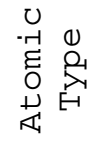

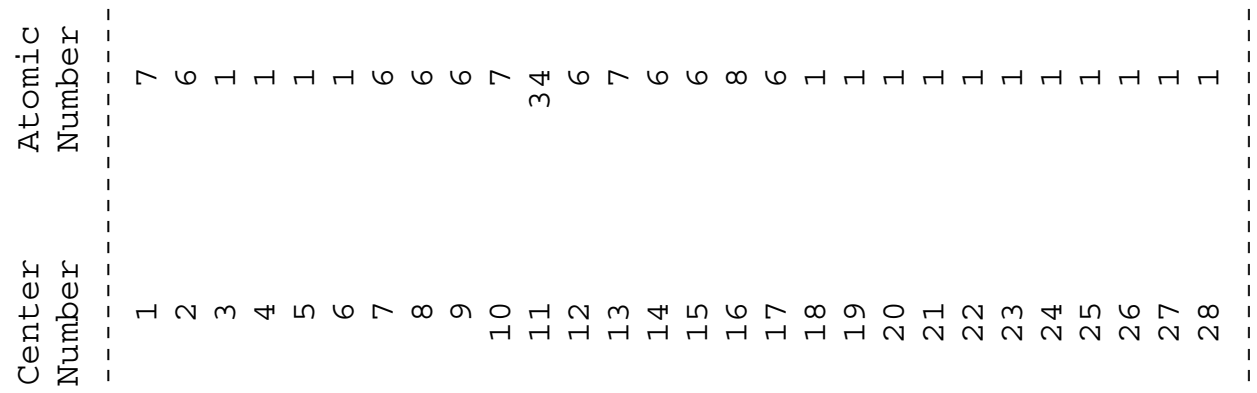

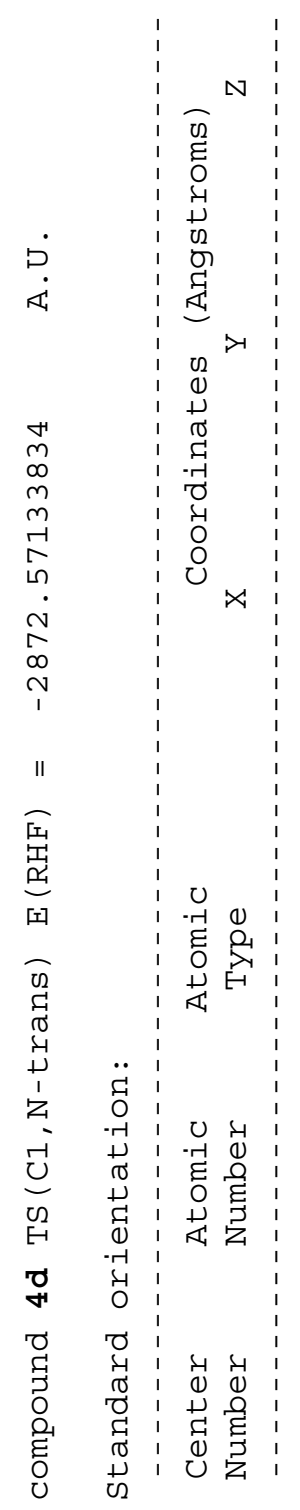




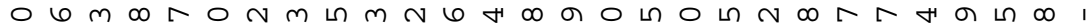

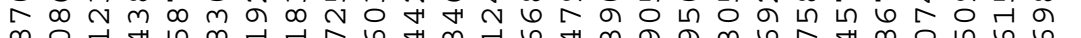
$\infty \quad 0{ }^{4}$

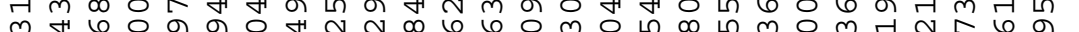

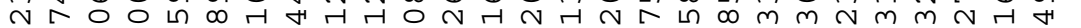

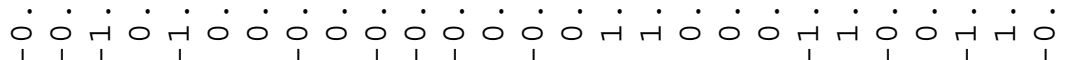

の

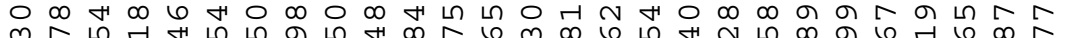

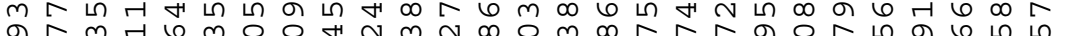
ब 0 ○ ब

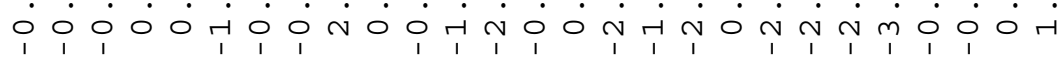

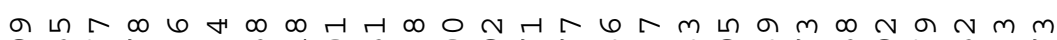

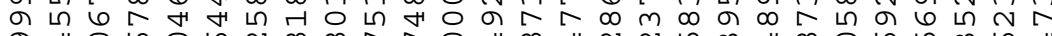
の

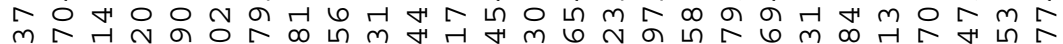

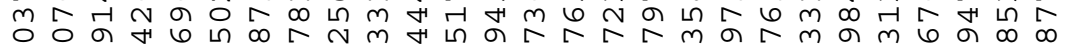

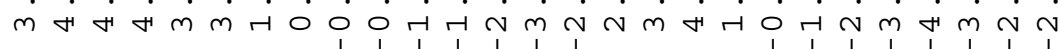

000000000000000000000000000

ヘ

ㅅ

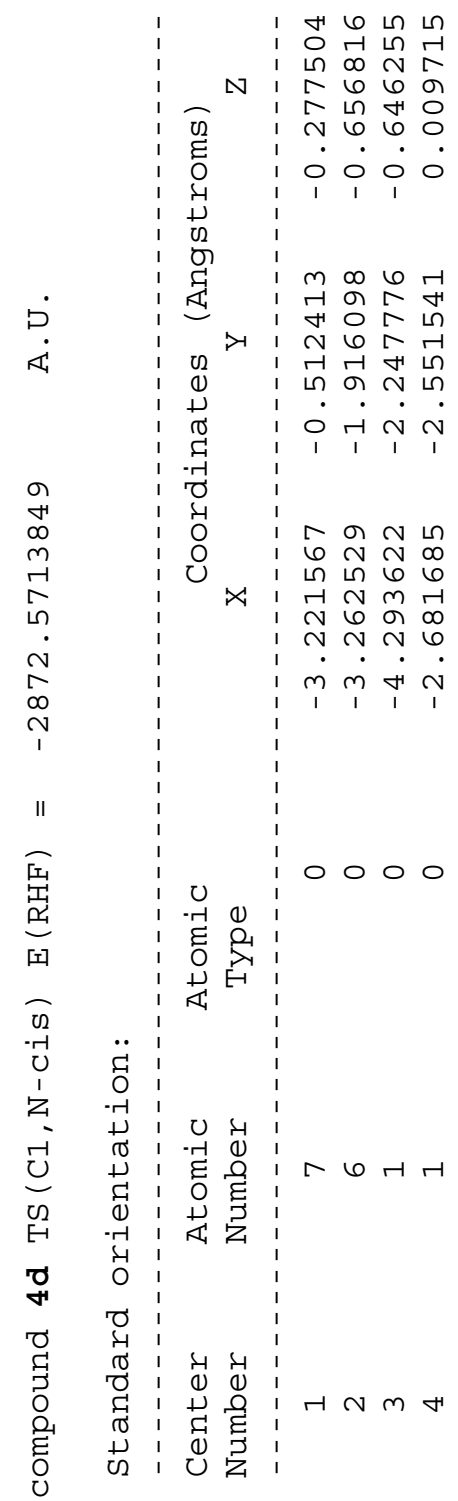




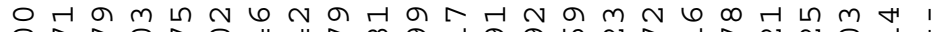
O

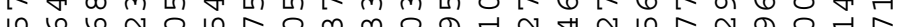
6 เ

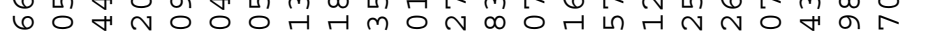

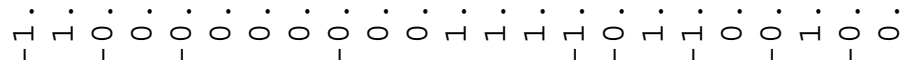

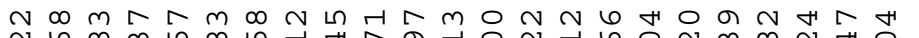

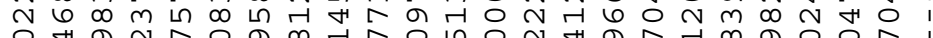

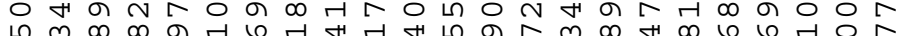

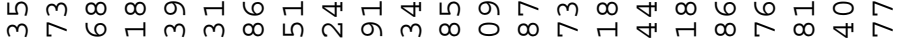
$0 \sim 0 \mathrm{~m} \sim \mathrm{m}$ ॠ

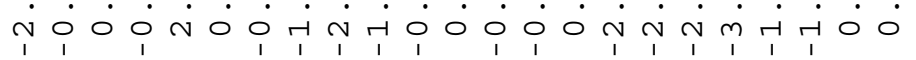

$\infty$ a 2 a

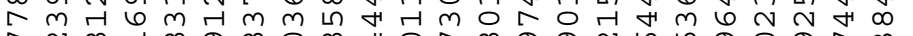

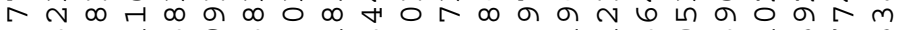

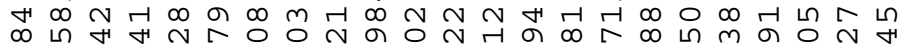

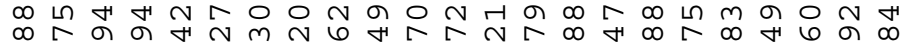

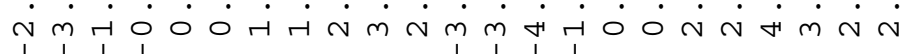

00000000000000000000000 4

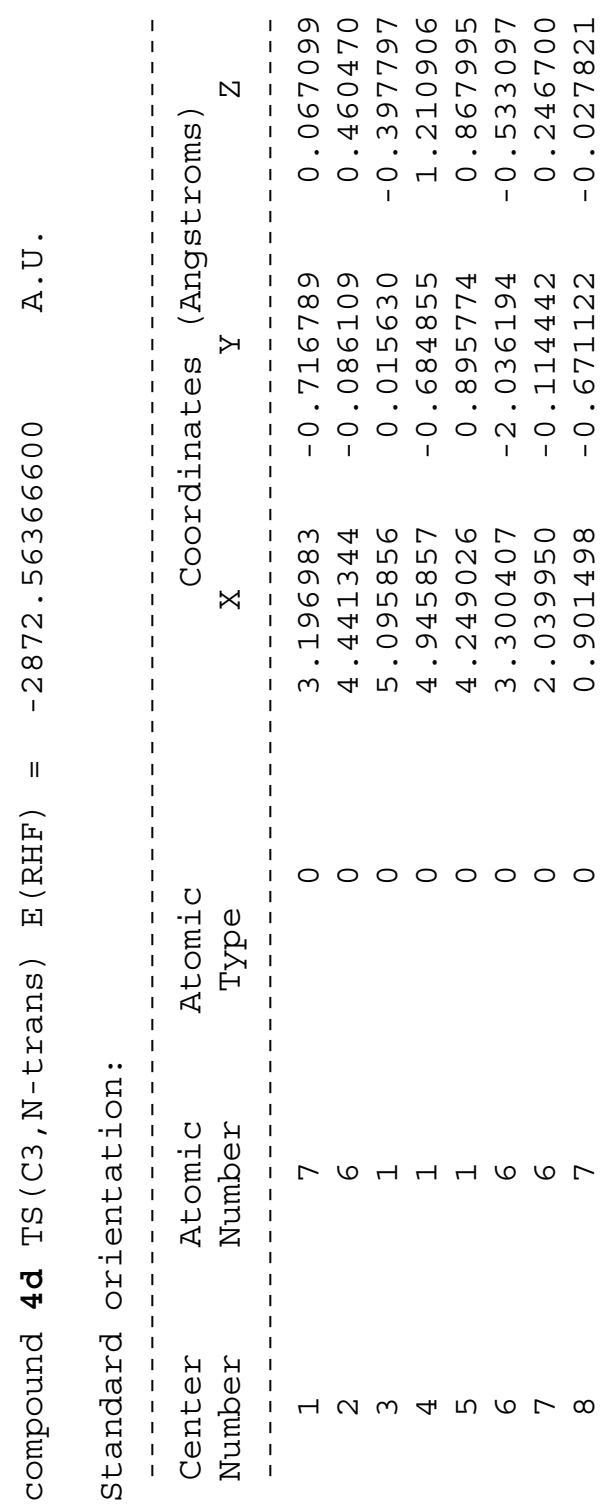




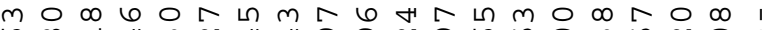

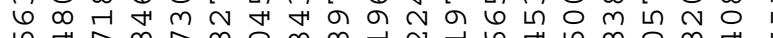

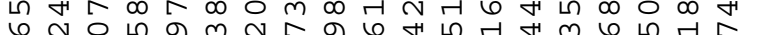

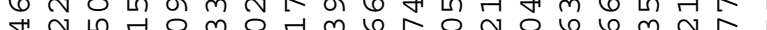

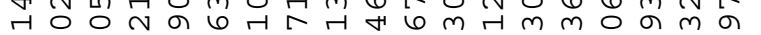

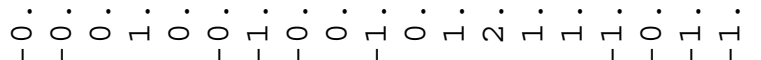

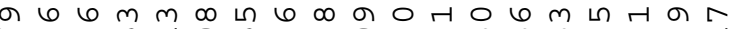
6 \&

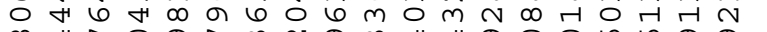

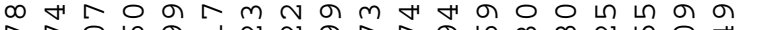
$\pi-06$ ब

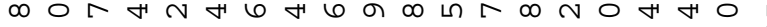

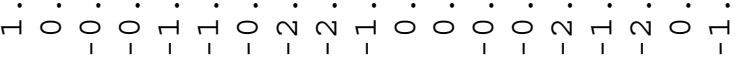

म 0 H

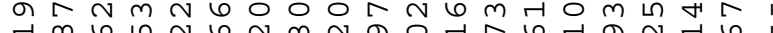

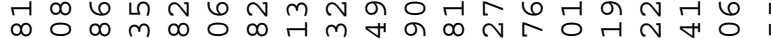

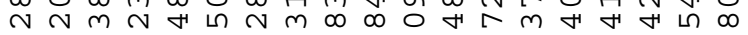

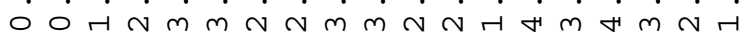
0000000000000000000 म

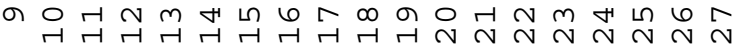

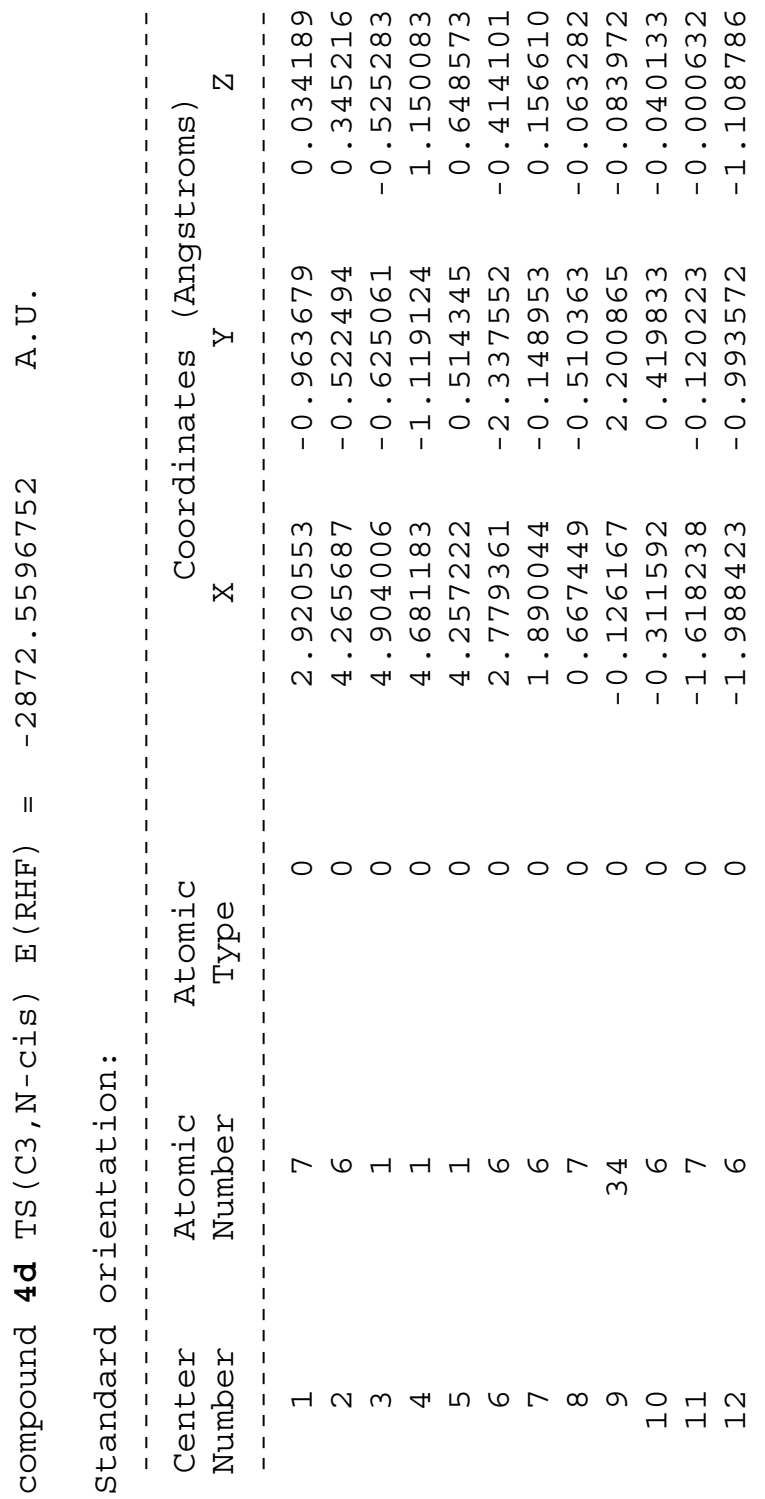


ด 6 6

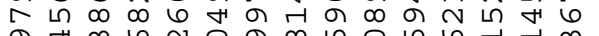

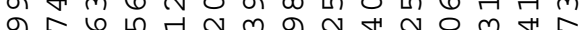

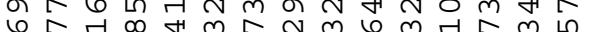

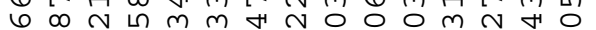

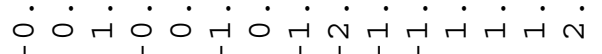

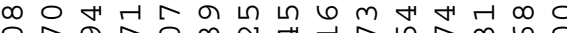

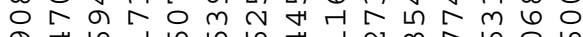

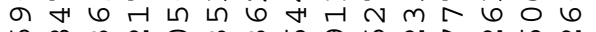

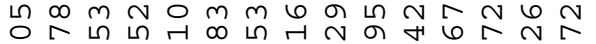
ए

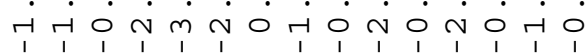

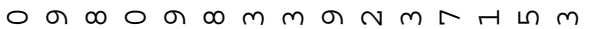
$\infty$ म 6 ○ 0 म O $\infty \mathrm{m}$ अ अ

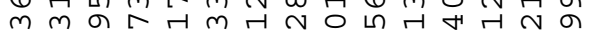
$\dot{m} \dot{m} \dot{r} \dot{m} \dot{m} \dot{r} \dot{N} \dot{m} \dot{m} \dot{\vec{r}} \vec{r}$ 000000000000000 6

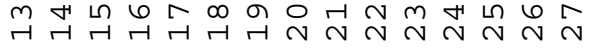

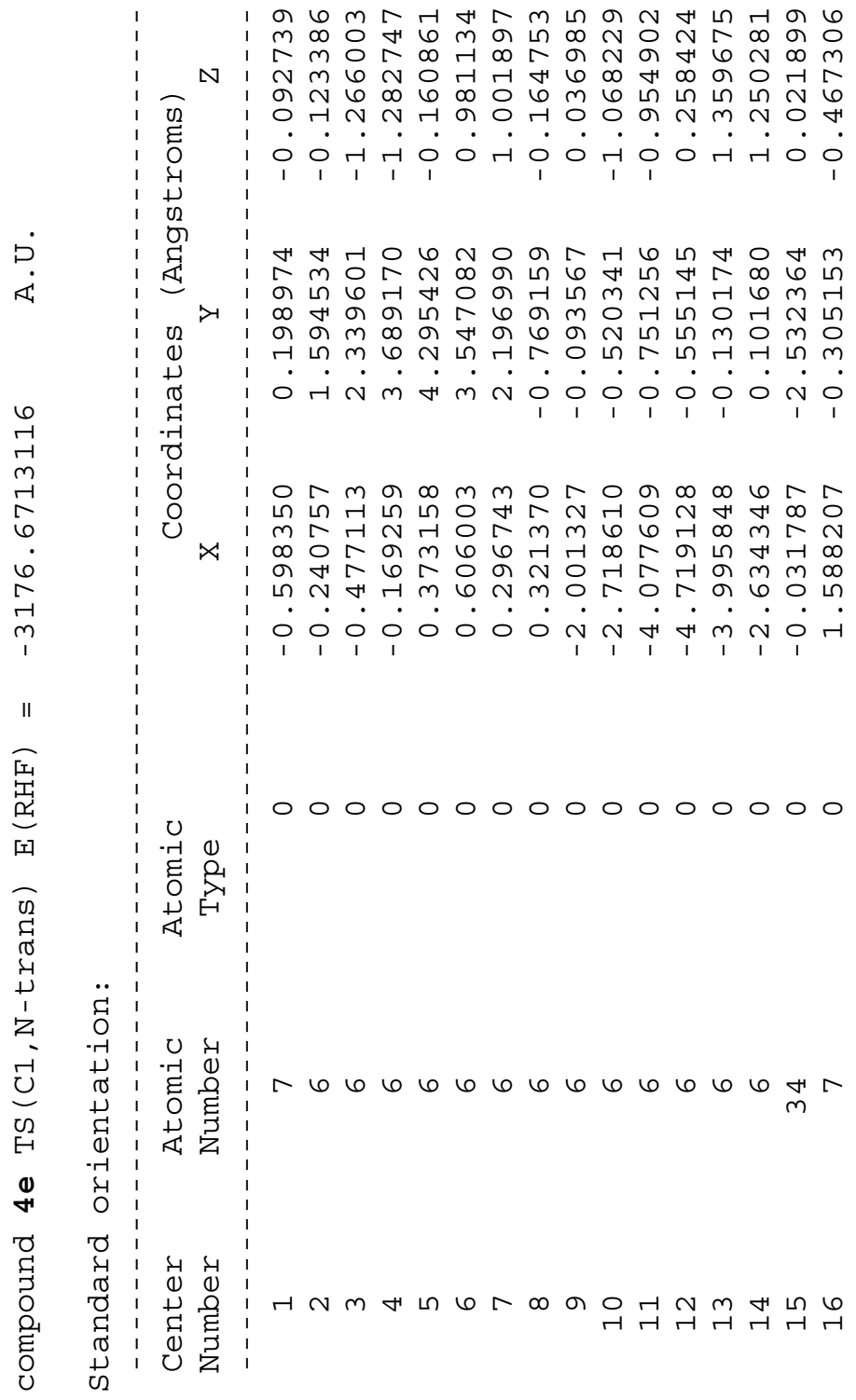


m

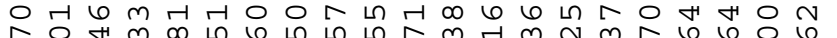

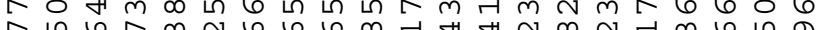

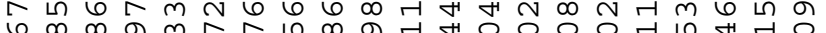
$-r \infty$ ए

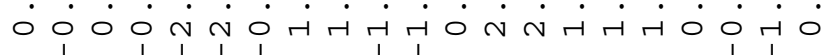

ヘ の の $4 N \infty 0$ ก 0 H

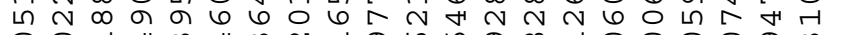

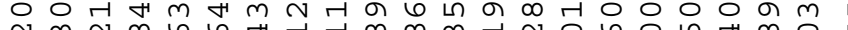

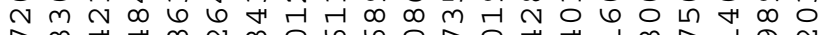

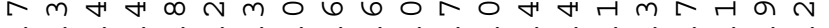

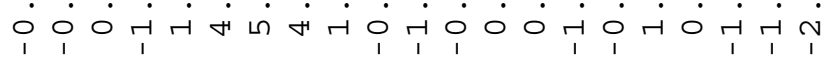

moG 0 H

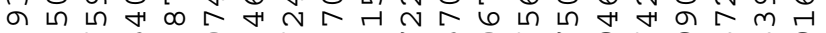

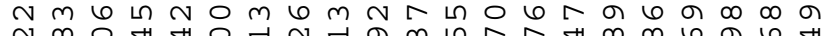

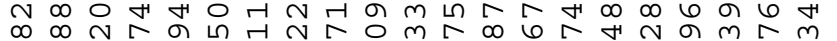

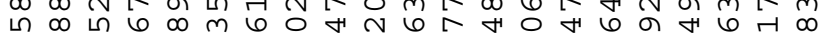
n m r 000000000000000000000

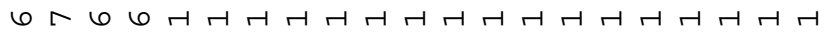

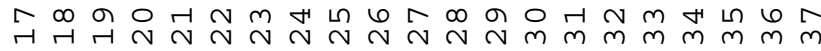

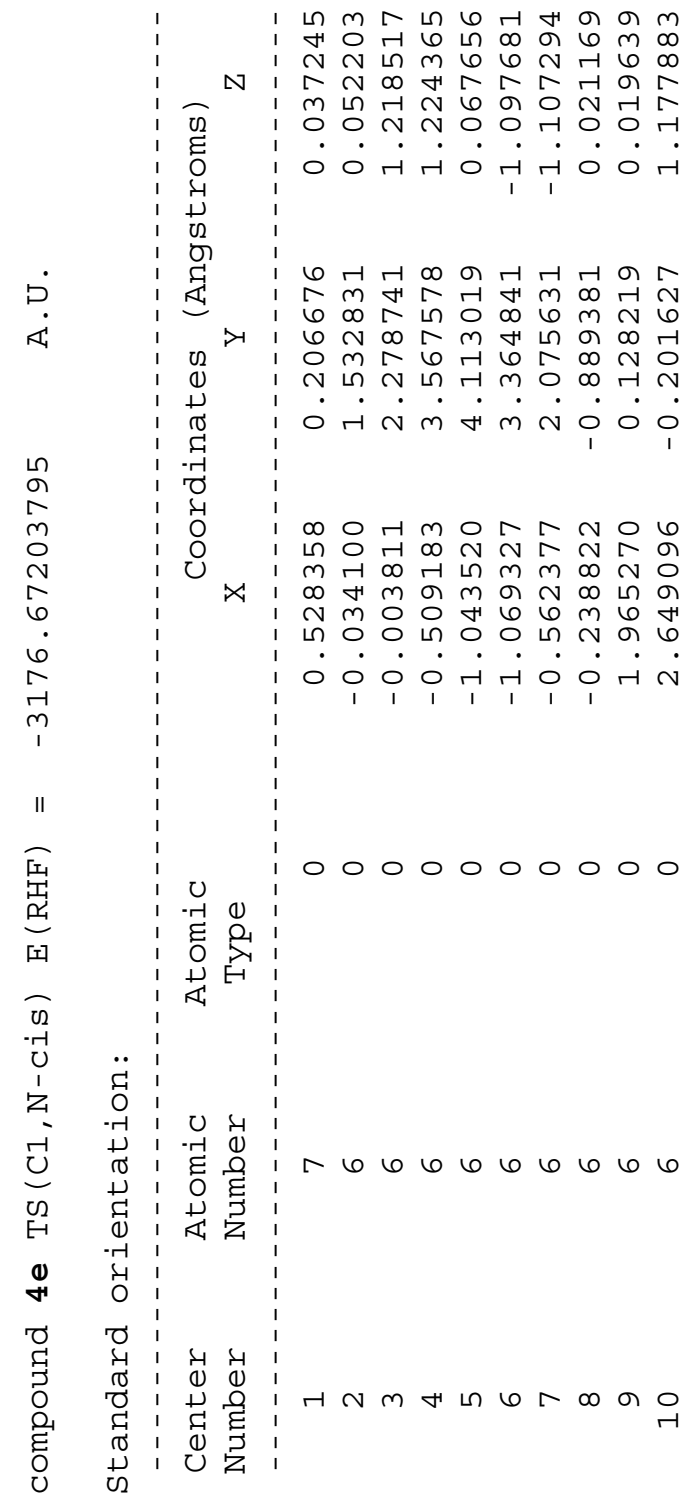


ब $N$ तु 6 다

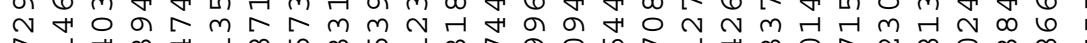
$N$ H

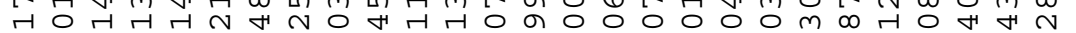

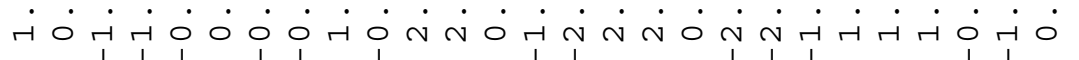

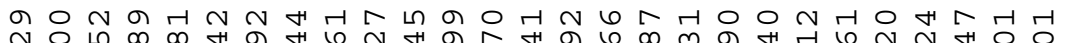

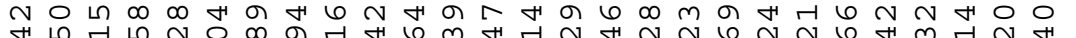

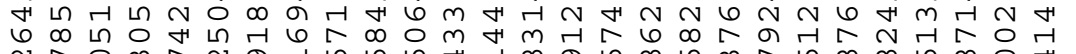
N 0 m $\mathrm{m} N$ ब N 0 H

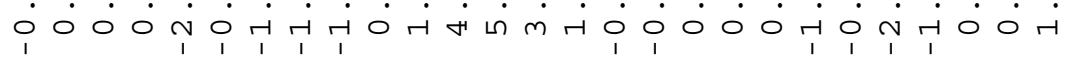

ம H $\infty m 6$ H $\circ \sim$ H $N$ H $N$
$m$

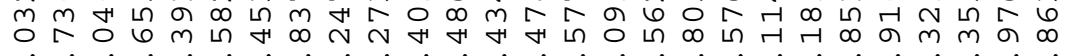

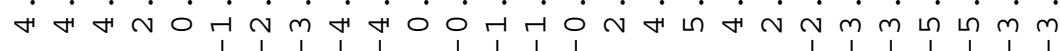

000000000000000000000000000

6 6 6

각다

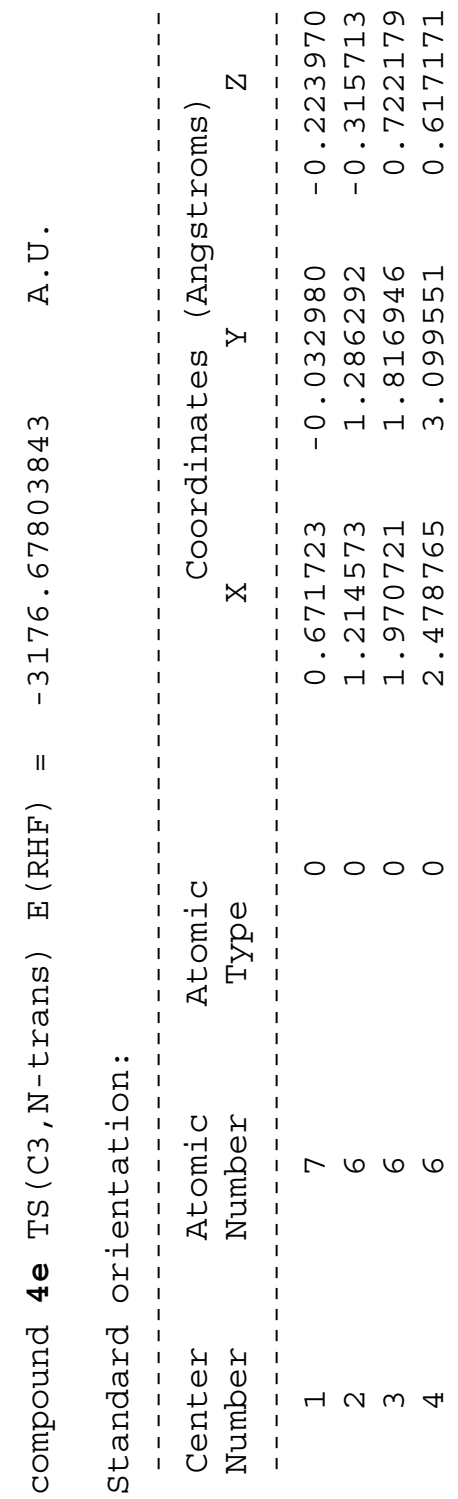


G 6 L $0 N 0$ N

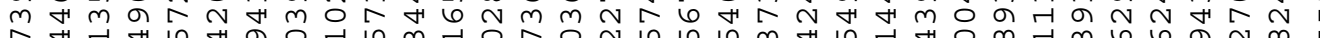

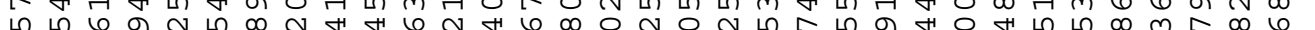

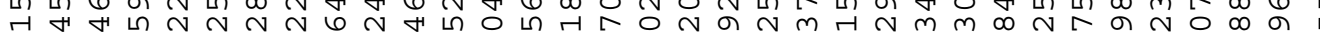
ही เ ○

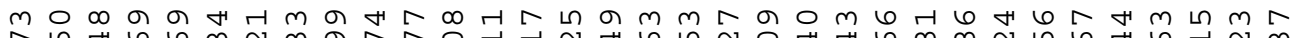
$\hat{N}$ म

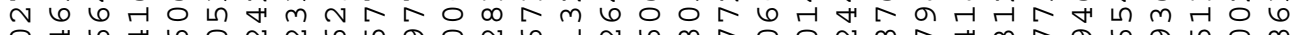

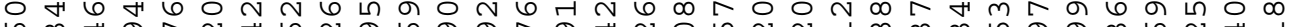

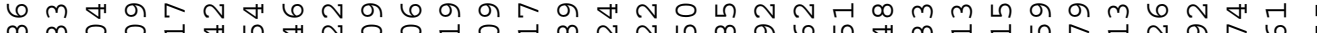

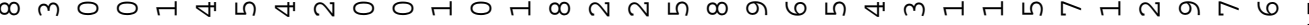

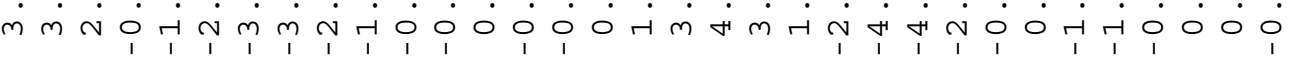

Ar

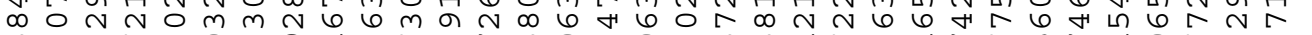

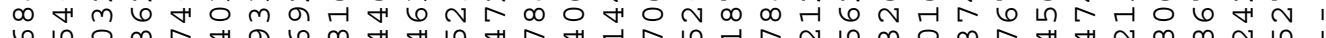
뉴

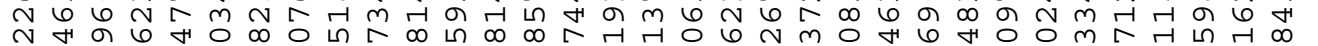

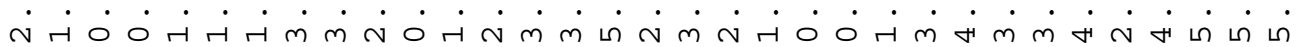
000000000000000000000000000000000

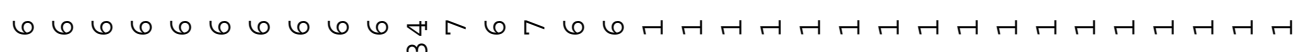
๓ $\wedge$ の 
I $m 6$ H

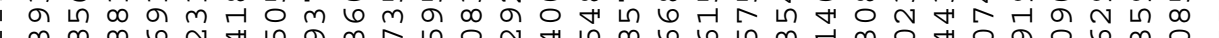

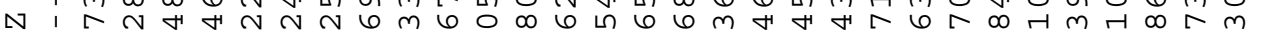
$N$ ก i $\infty 6$, l.

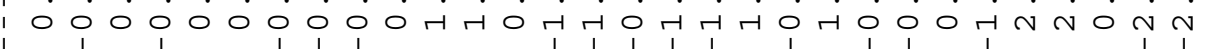

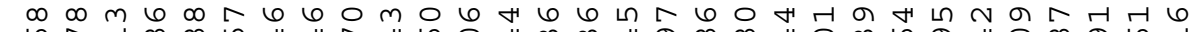
GN L H

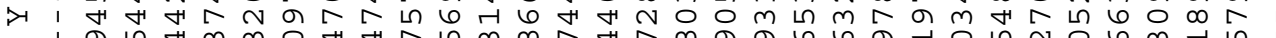
ब 16 เ

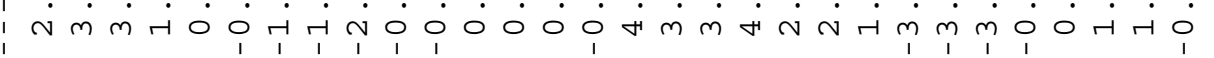

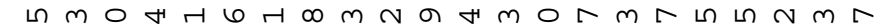

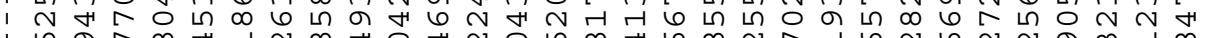
$x$, ○ स H ON

\section{है} ^

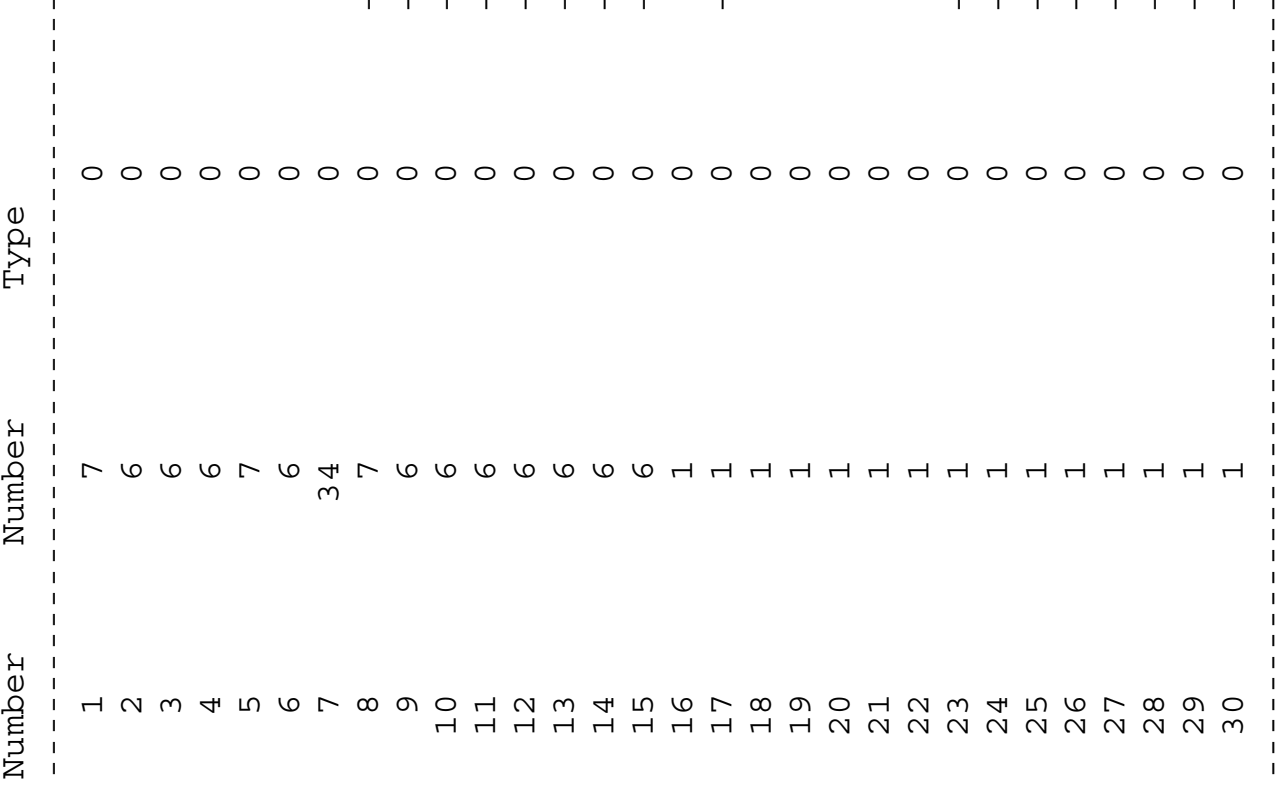




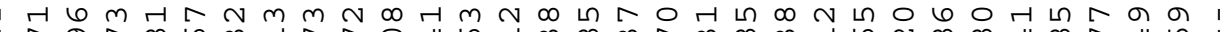

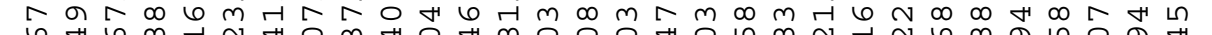

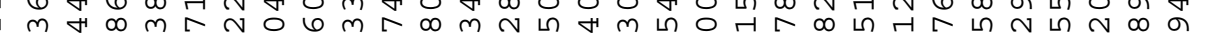
เ $m$ -

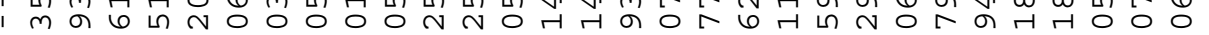

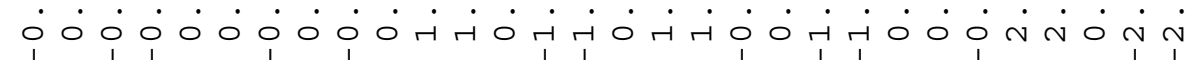

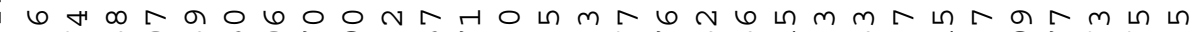

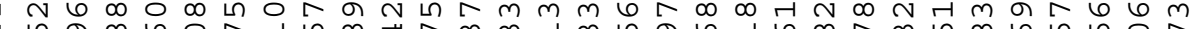
1 ம HN $N$ F

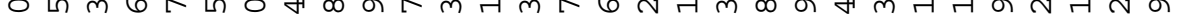

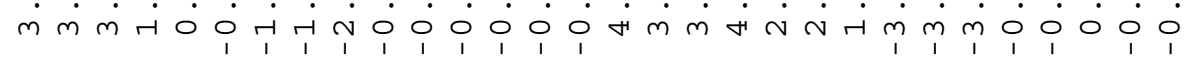

○ $m N$ H

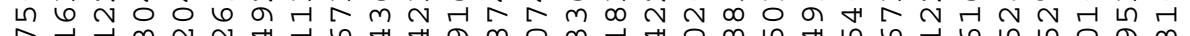

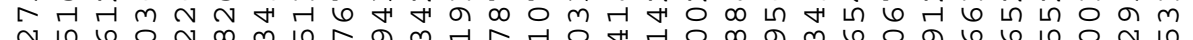

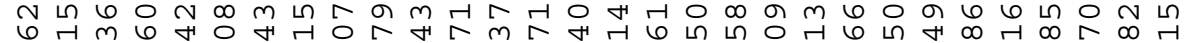

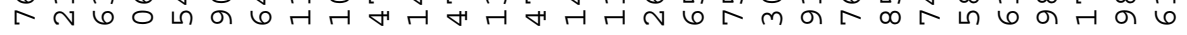

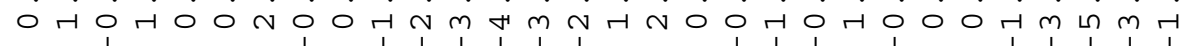
000000000000000000000000000000 ก m H

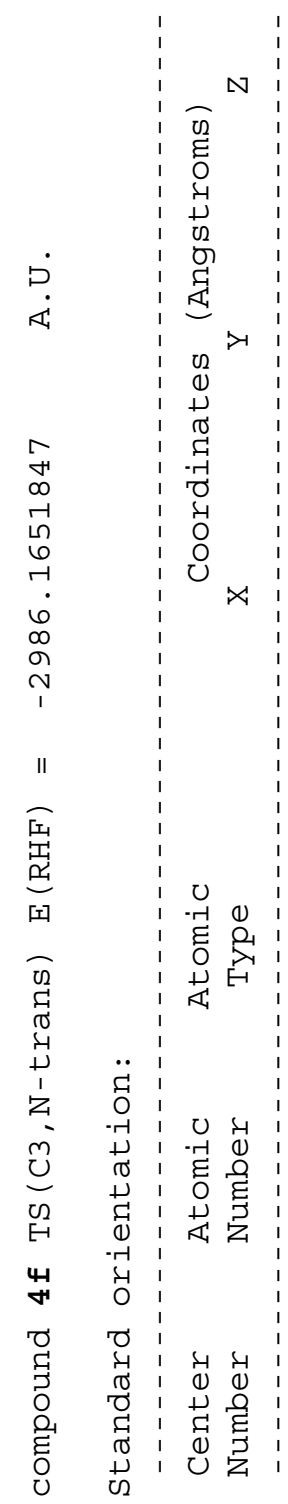


の

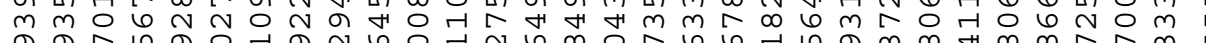

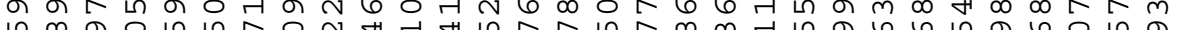
n 0000

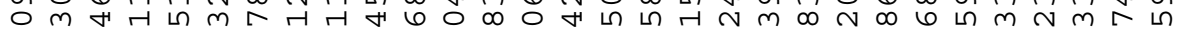

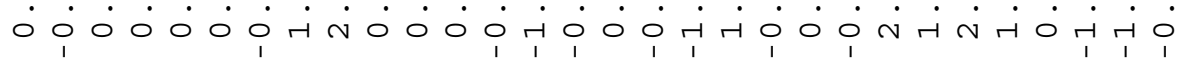

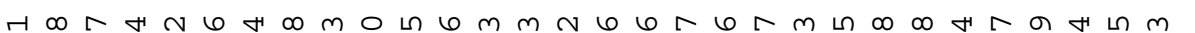

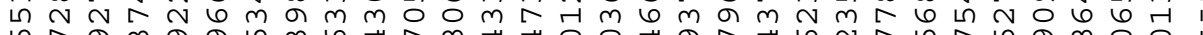
เ

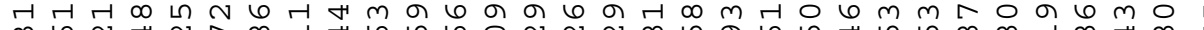

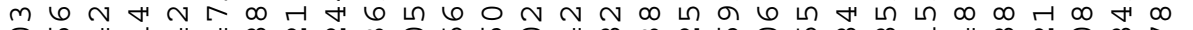

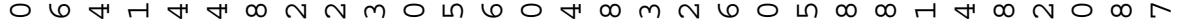

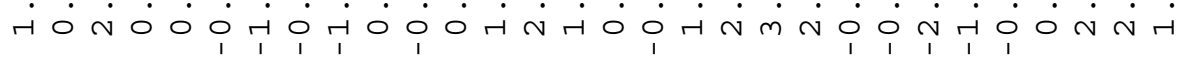

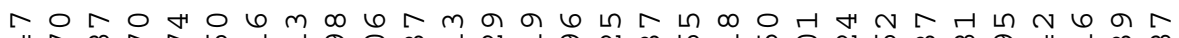
Н

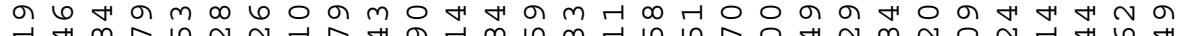
ने

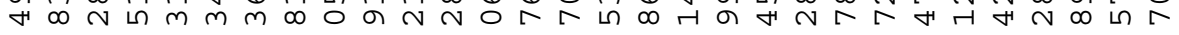

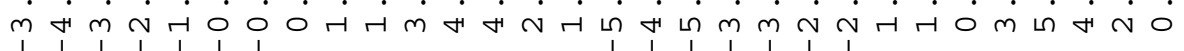
000000000000000000000000000000 ค H 2 य

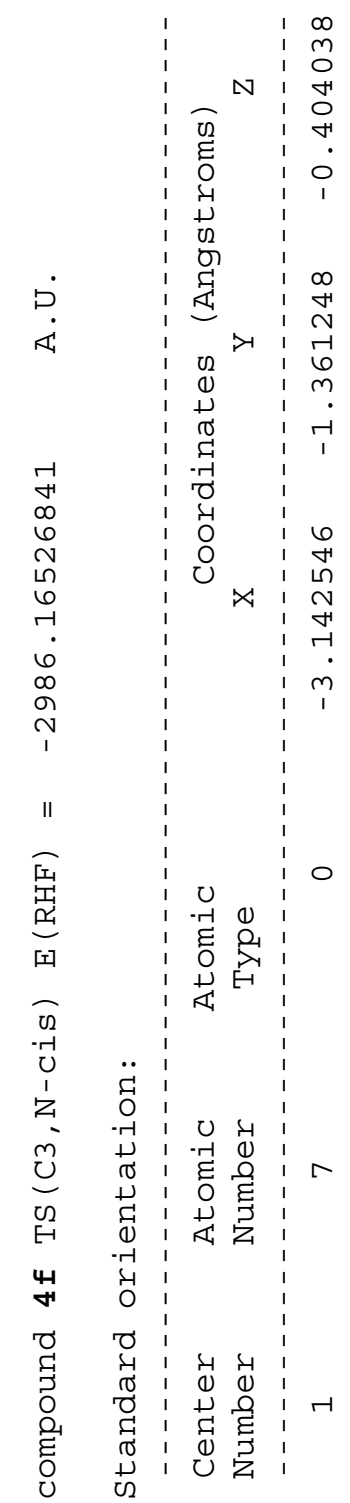




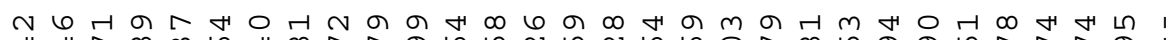
H

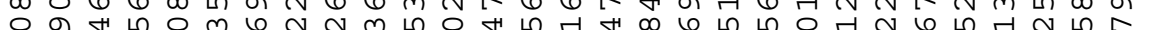
m $m+m$ म

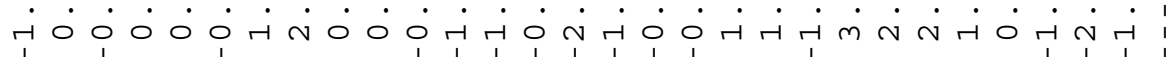

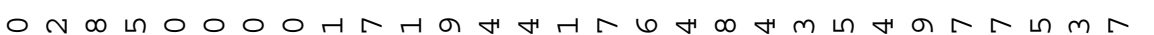

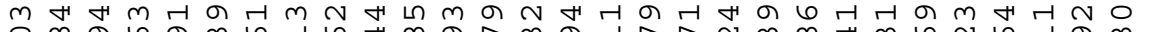

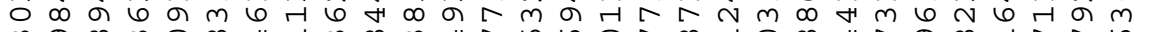

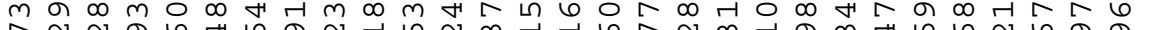
$\hat{N} N$ a 6 म

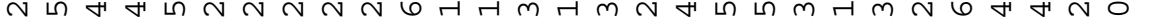

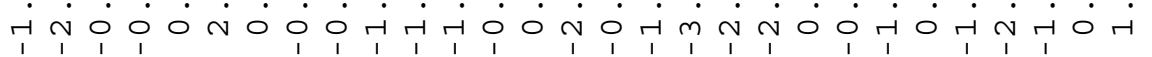

H 40 L

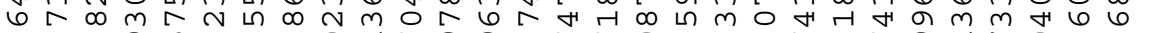

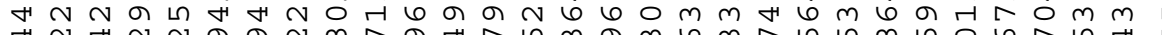

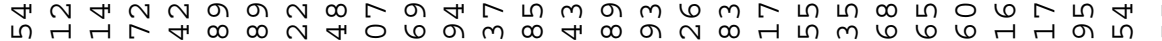
N

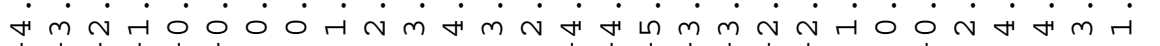

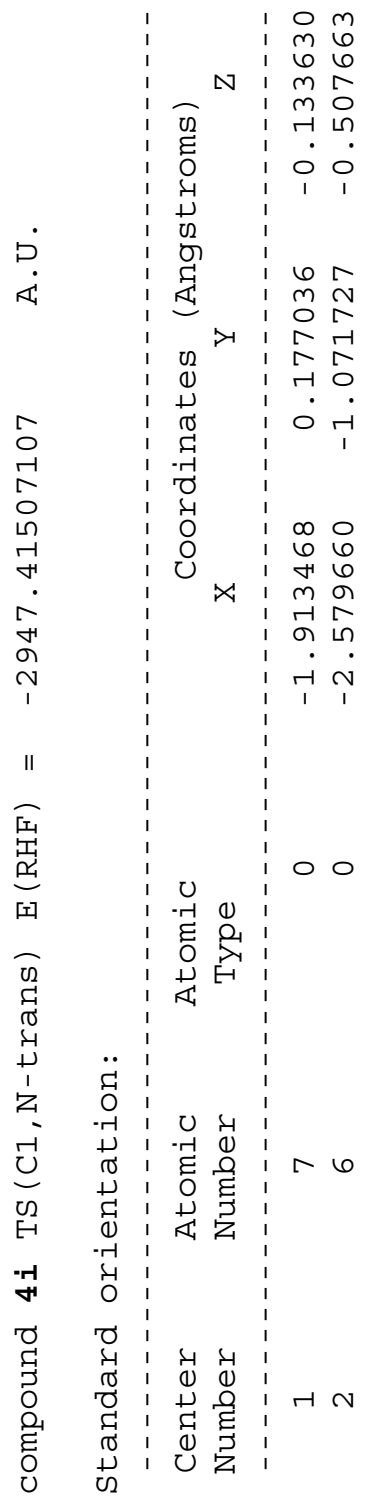


几 $m$ त

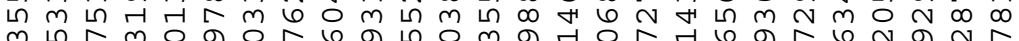
$-4000$ பn $N$ $m$ i $m$ त $\infty$ ब

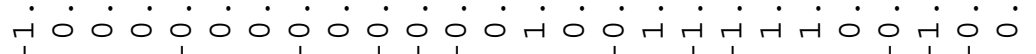

0 त 0 -

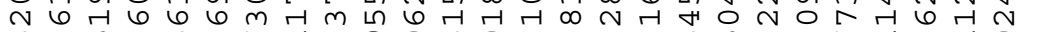
$\infty$ \&

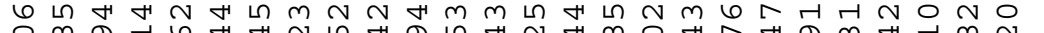
0 の

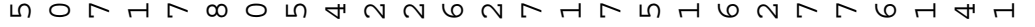
ت

N

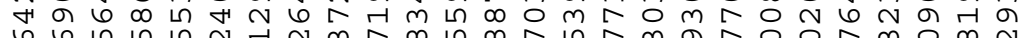

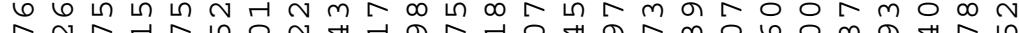
ก

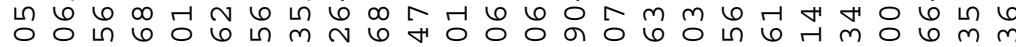
席 00000000000000000000000000 न m

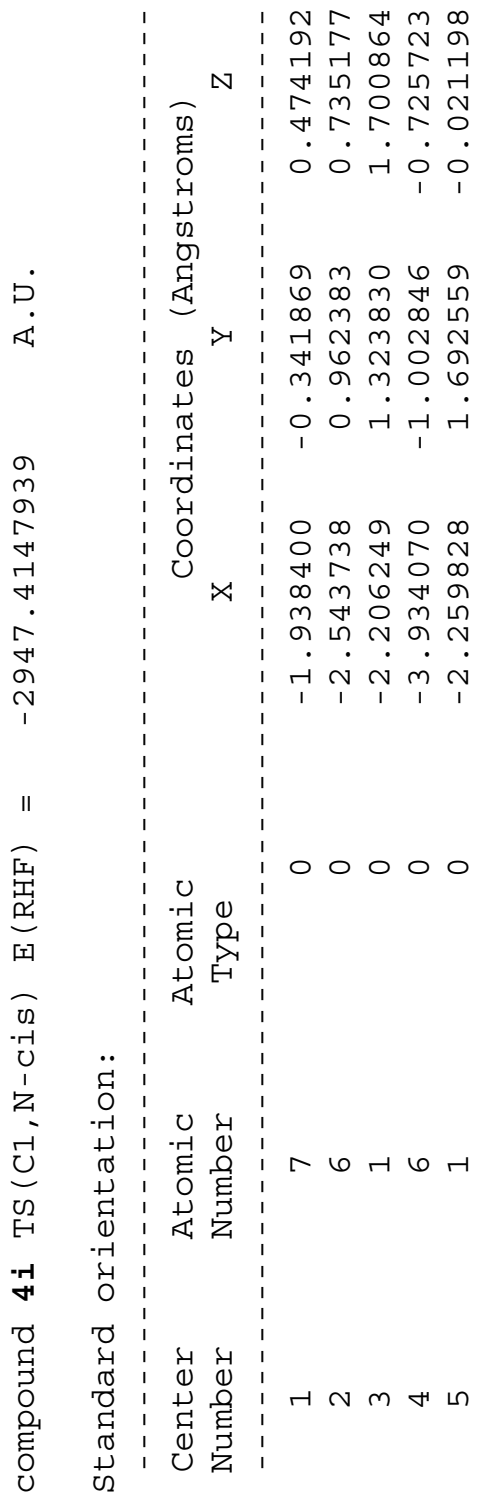




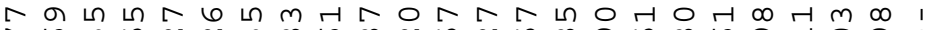

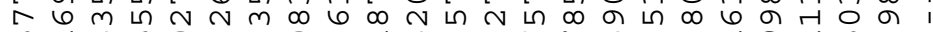

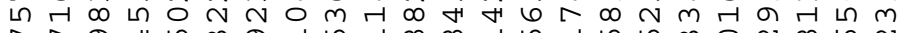

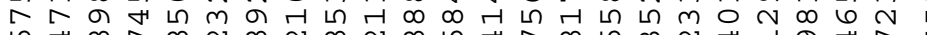
L

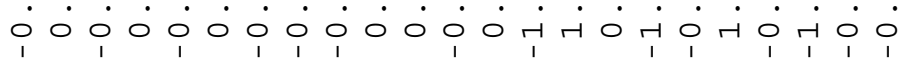

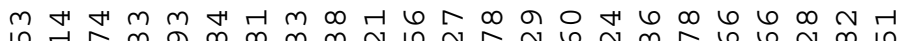
L

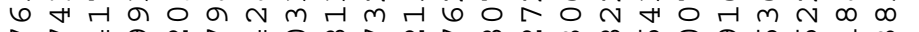

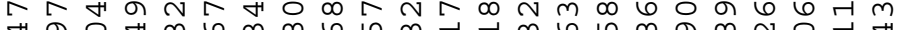
r

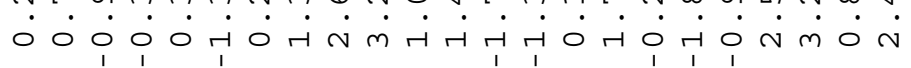

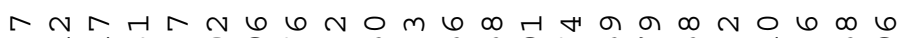

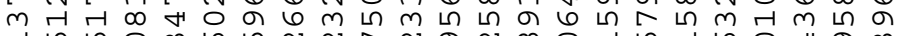

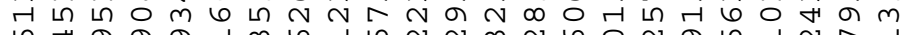

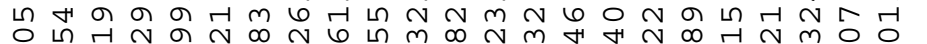

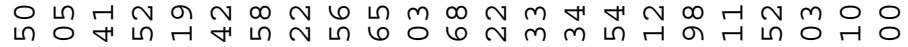

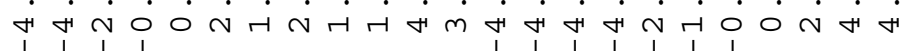
00000000000000000000000

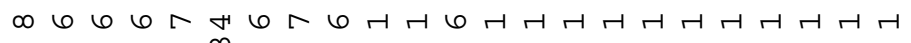

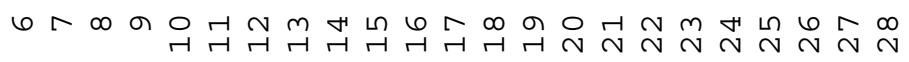

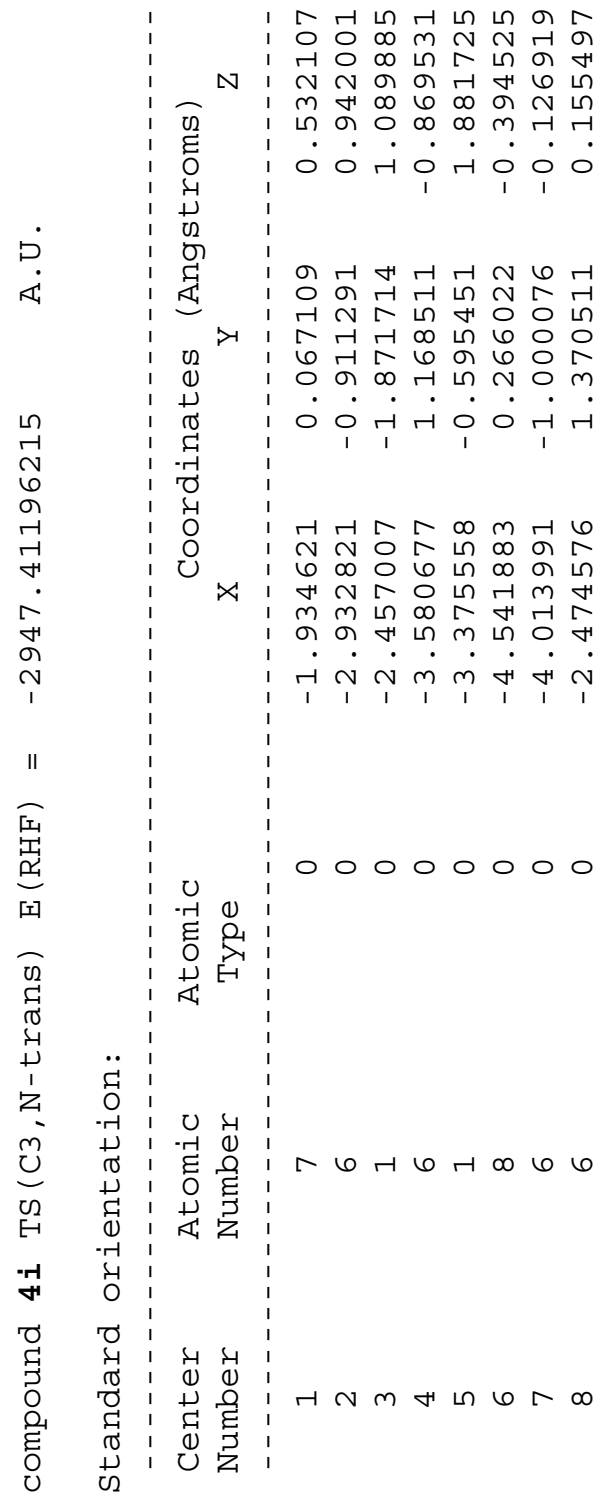


$\infty$ ॠ

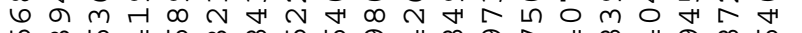

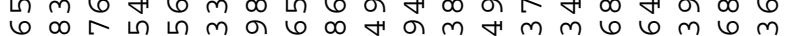

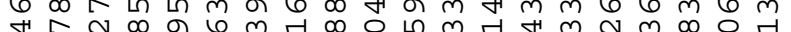
เ $N m 0$ r 0 ब

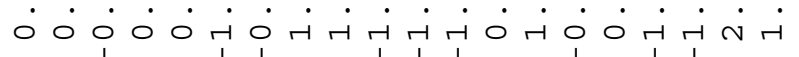

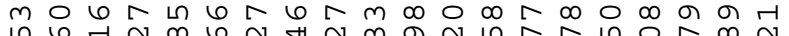

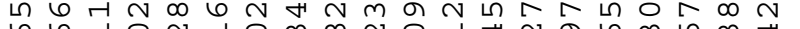

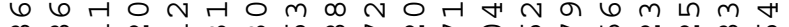

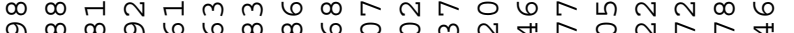
$-100$

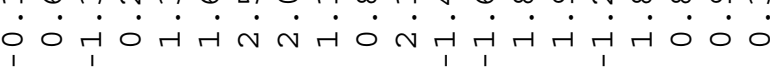

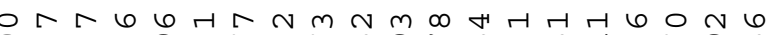

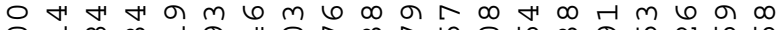
$\circ$ ㄷ $m$ 가

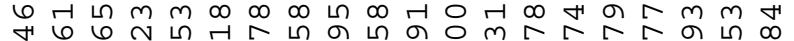

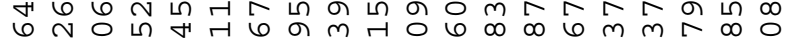
$\dot{0} \dot{0} \dot{\sim} \dot{\sim} \dot{m} \dot{m} \dot{m} \dot{m} \dot{m} \dot{m} \dot{\sim} \dot{\sim} \dot{0} \dot{\sim} \dot{m} \dot{N}$ 00000000000000000000 שก

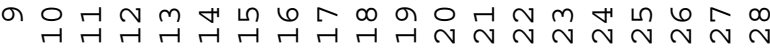

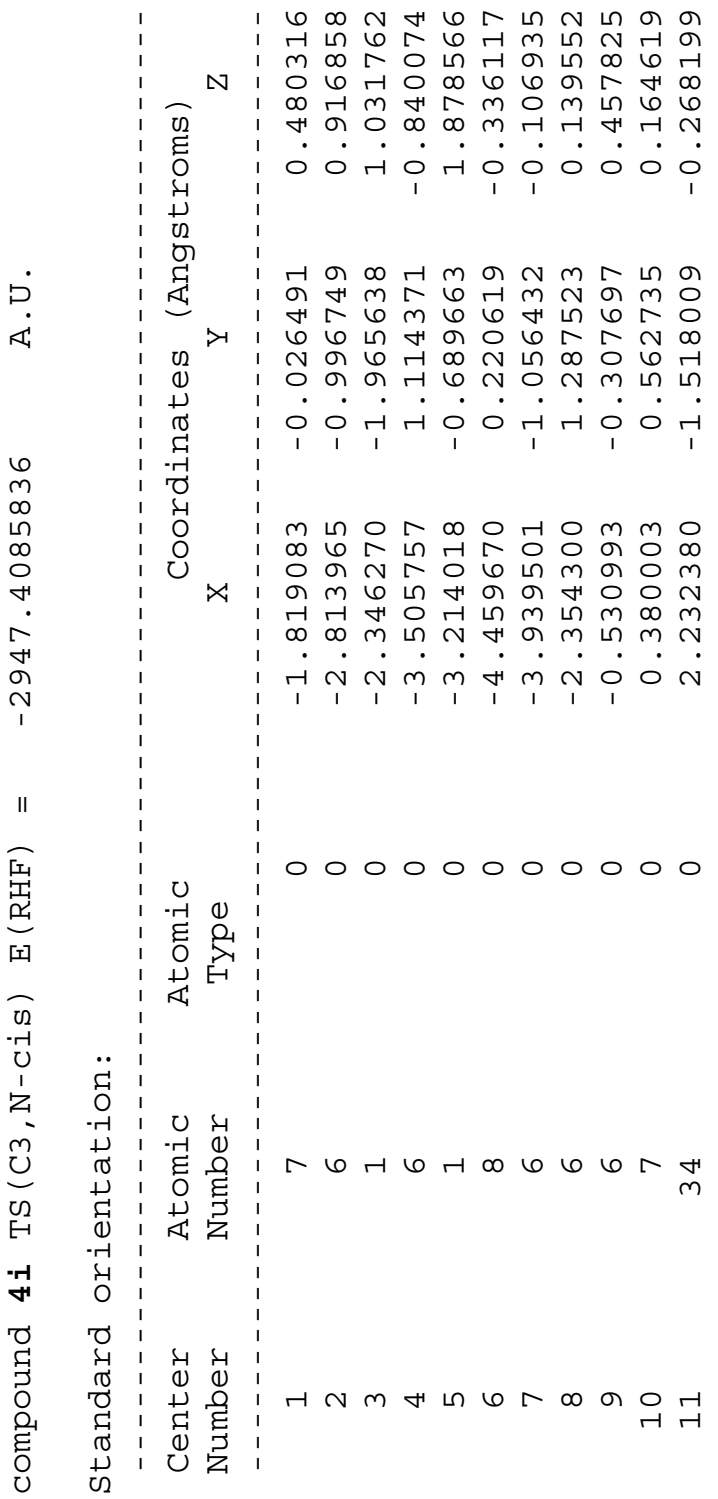


4m $N m \sim H m$ ब $N$ ब ○

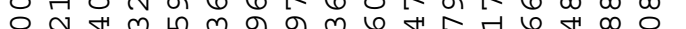

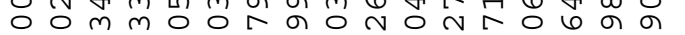

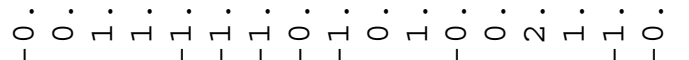

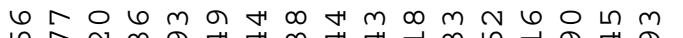
IN $N m$ ब 6 H 6 ம 6 त

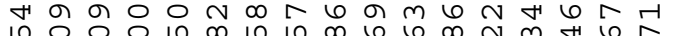

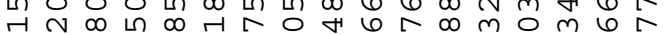

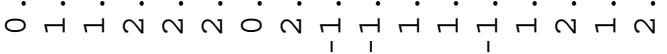

の $\infty m \infty 6$ H

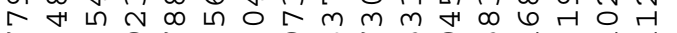

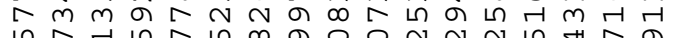

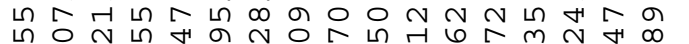

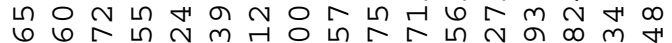

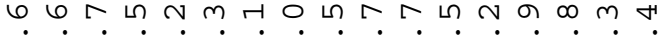

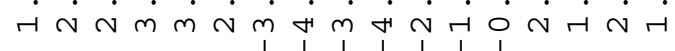
00000000000000000 $\curvearrowright$

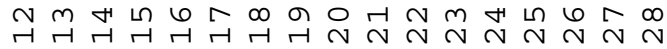

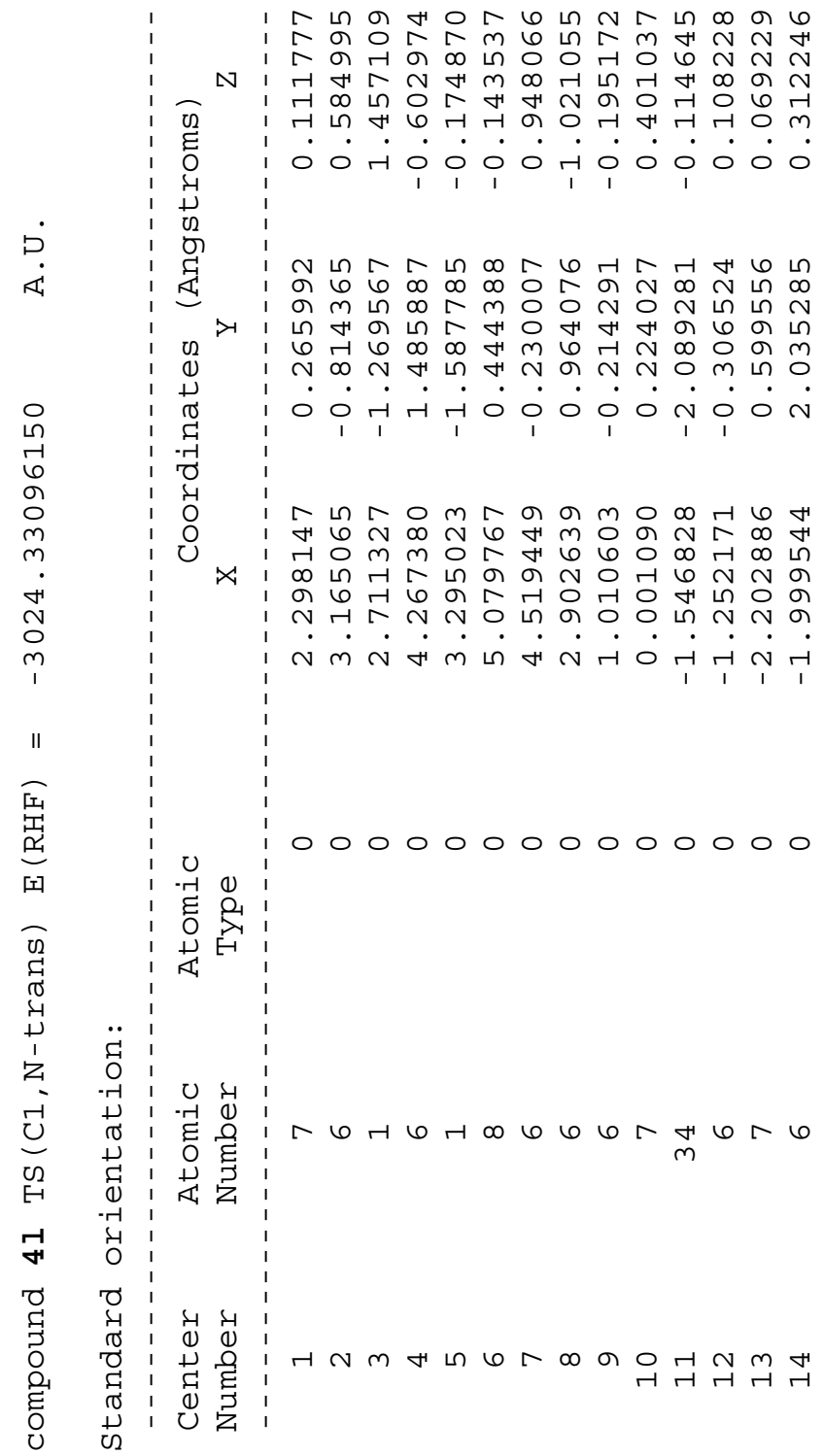

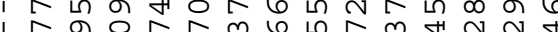
$1 \pi$ व

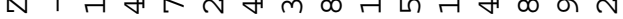

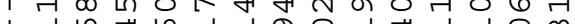
त म स

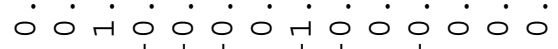

N 1 ก

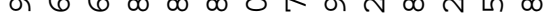

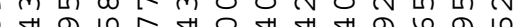

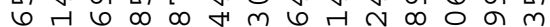

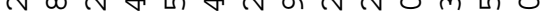

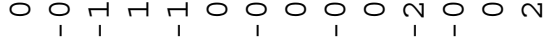

10 $\infty$ म N $\mathrm{N} N$ ก

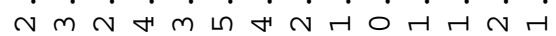


O $\mathrm{N}$ H 0 L $\mathrm{m} \omega \mathrm{H}$ H

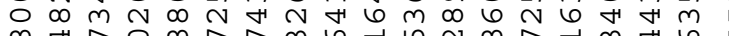
$\infty$ H

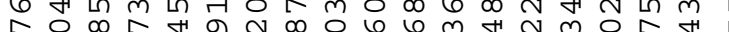

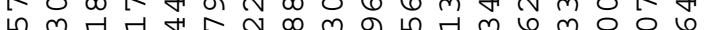

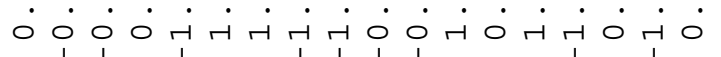

त

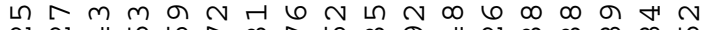

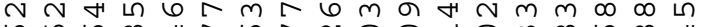

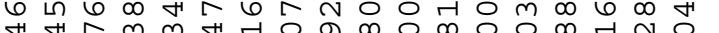

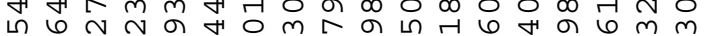

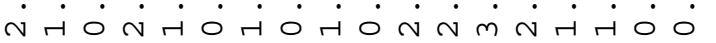

$m \sim N \sim m m N \infty \circ m \circ 6 N$ H

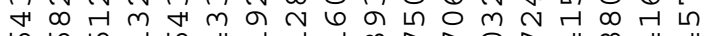
6
0 6 स

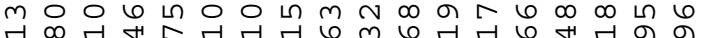

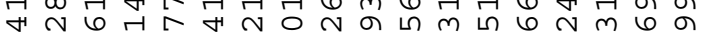

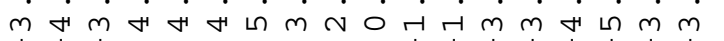

000000000000000000

6 6 भ

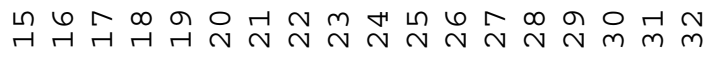

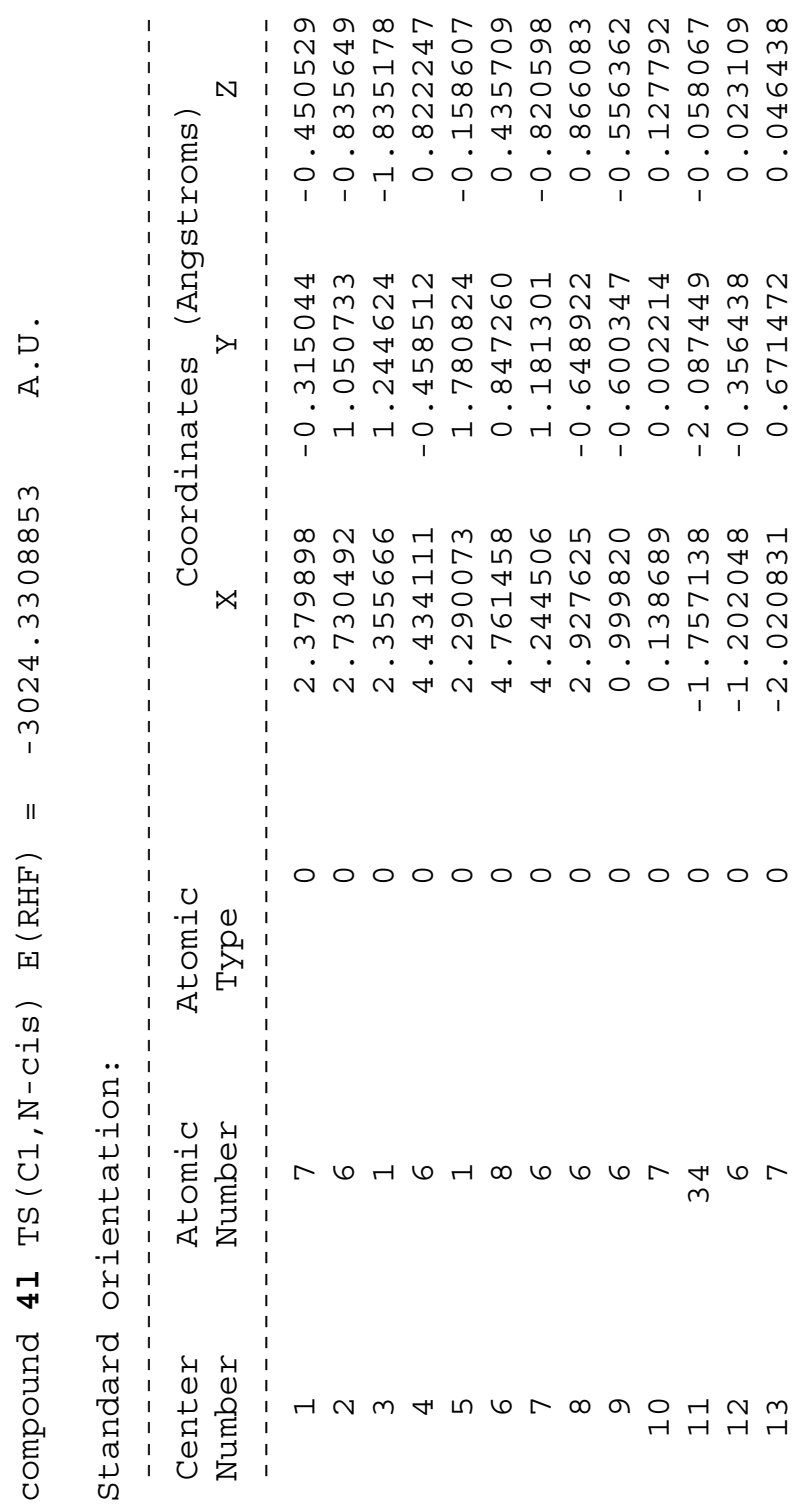


$m 0$ H N

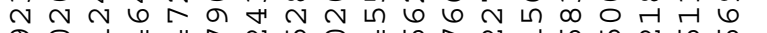

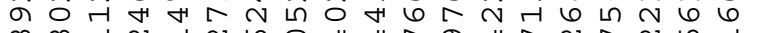
$\infty \quad \infty$ H $N$ H $N$ C 0 H

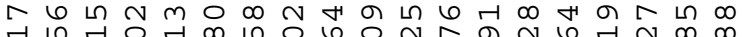

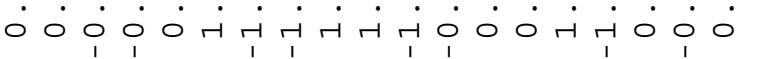

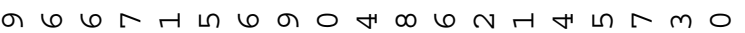

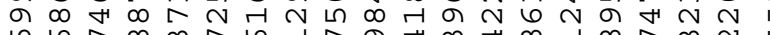

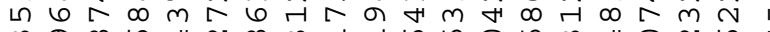

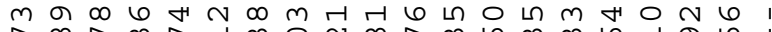

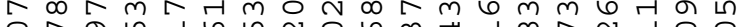

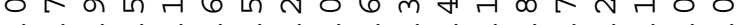

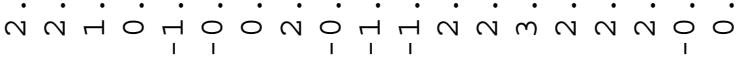

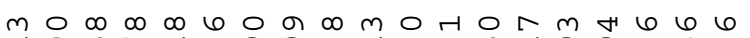

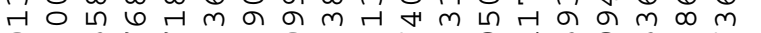

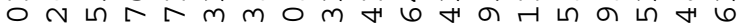
당 の

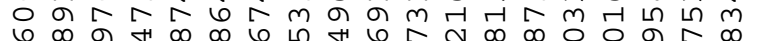
$6 \infty$ の $+\infty \infty 6$ म

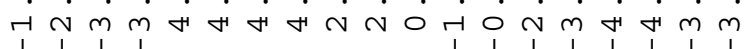

0000000000000000000

,

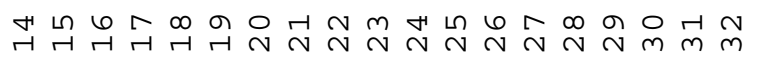

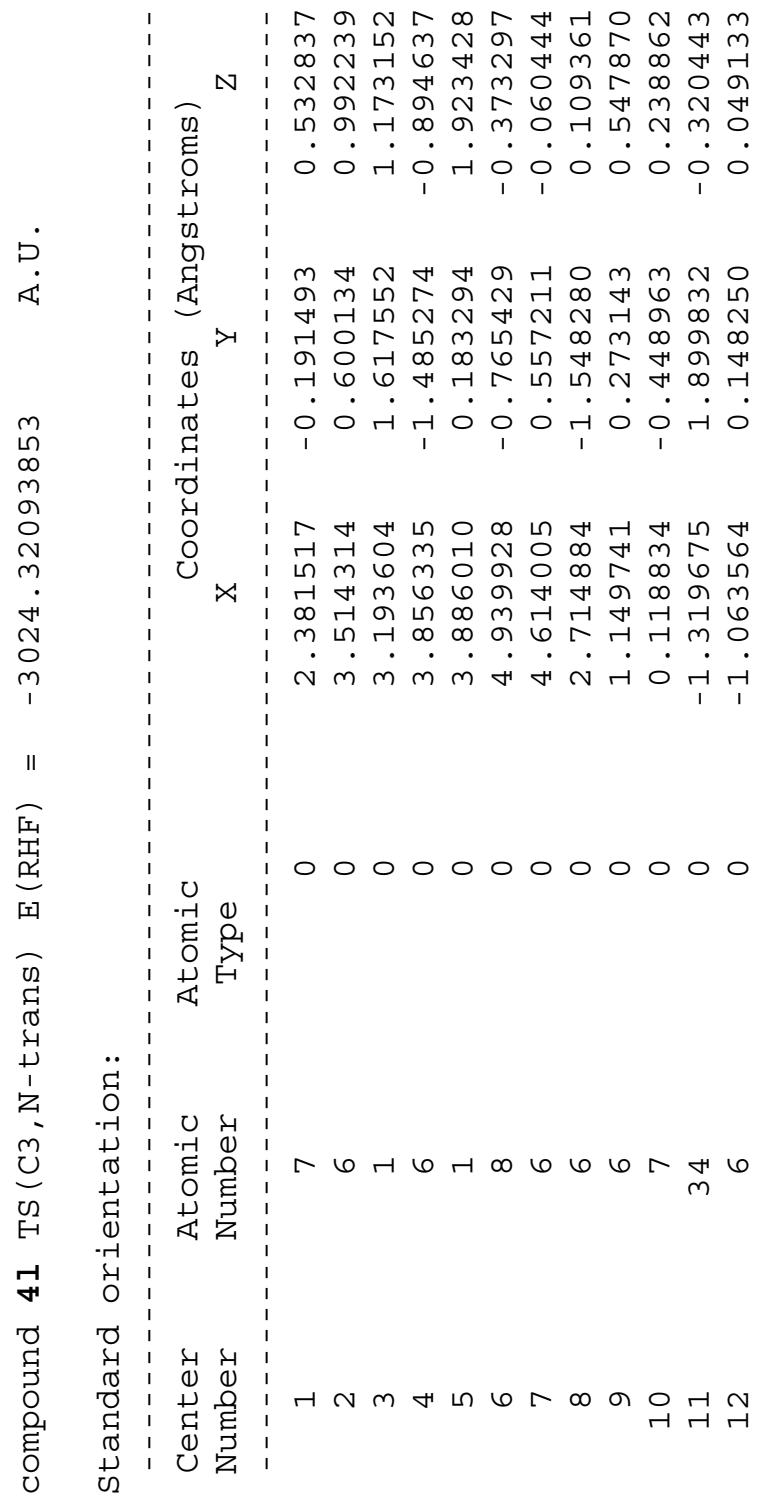

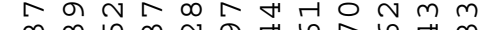
$\infty \sim m$ r $m$ a $\pi$ a $N 60 \%$ ० 0 में

$m+N$ H

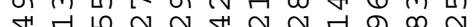
نं $\dot{0} \dot{0} \dot{0} \dot{0} \dot{0} \dot{0} \dot{0}$

(1)

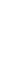

.




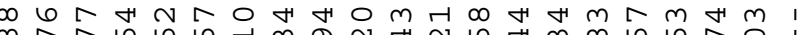

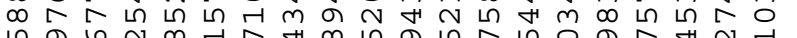

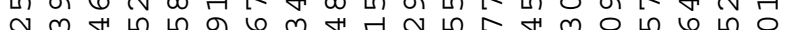

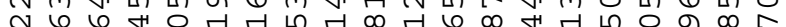
H

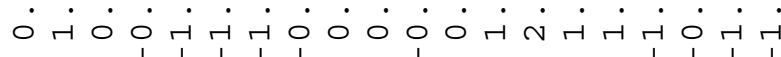

म $4 m m m \in$ H ○ ब $N$ त

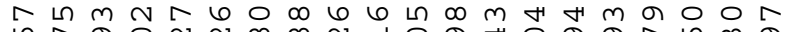

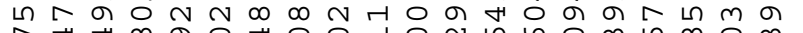
- 4 म

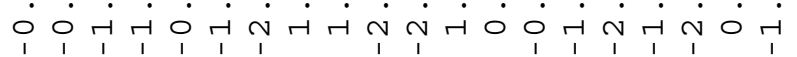

$m \infty$ の

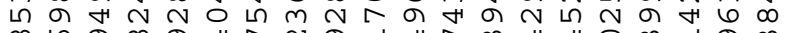
$\infty 6$ の $\infty$ ब

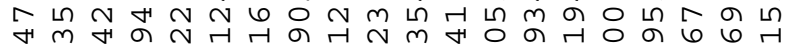

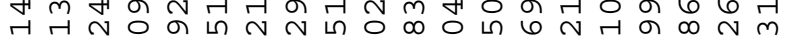

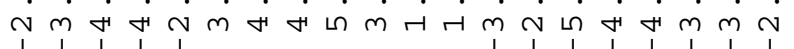
00000000000000000000 ऽ

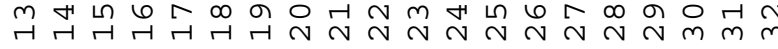

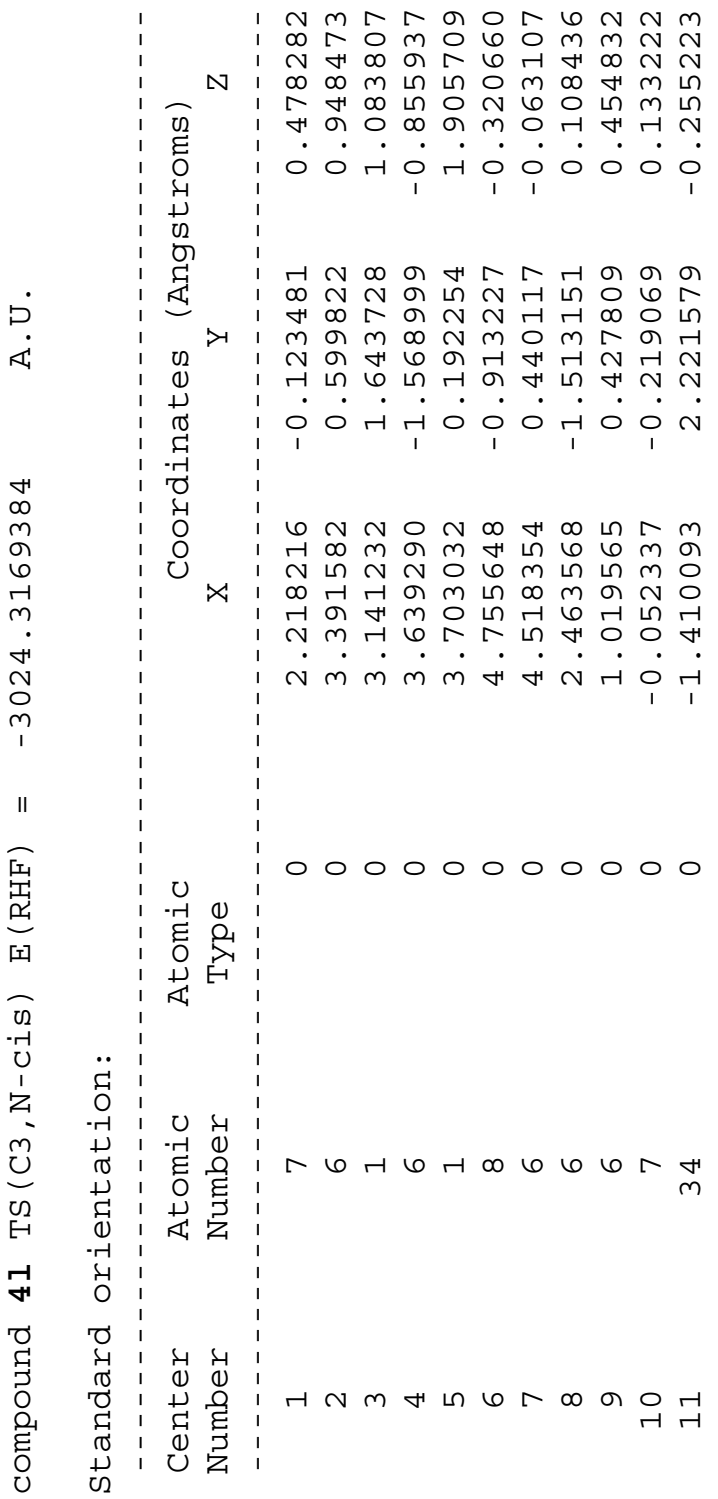


H $\infty$ H $\infty \mathrm{m} m \infty$ H m $m$ б

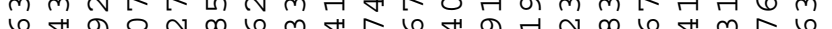

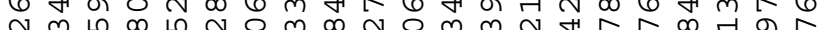

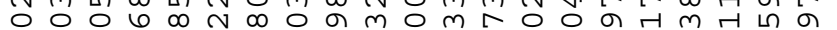

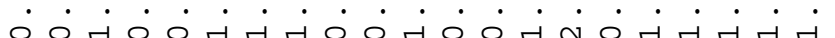
$\begin{array}{llllll}1 & 1 & 1 & 1 & 1 & 1\end{array}$

a 40 4

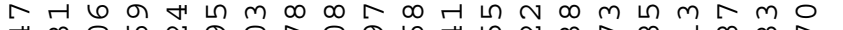

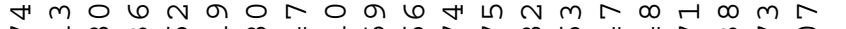
다 $\infty m 6$ 대

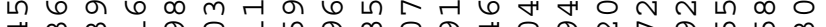

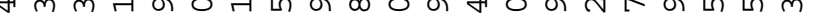

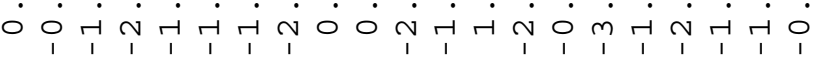

$m \circ 6$ H

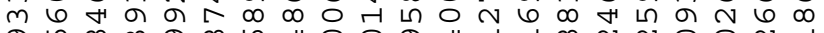

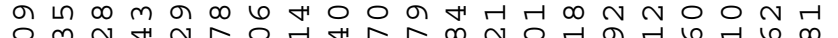

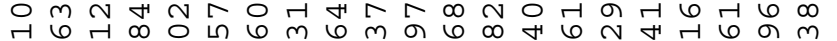

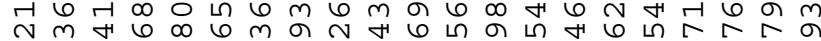

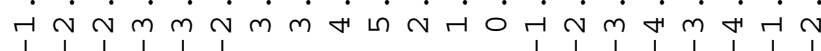

000000000000000000000

e

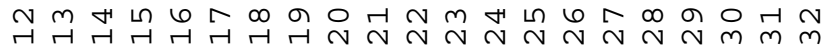

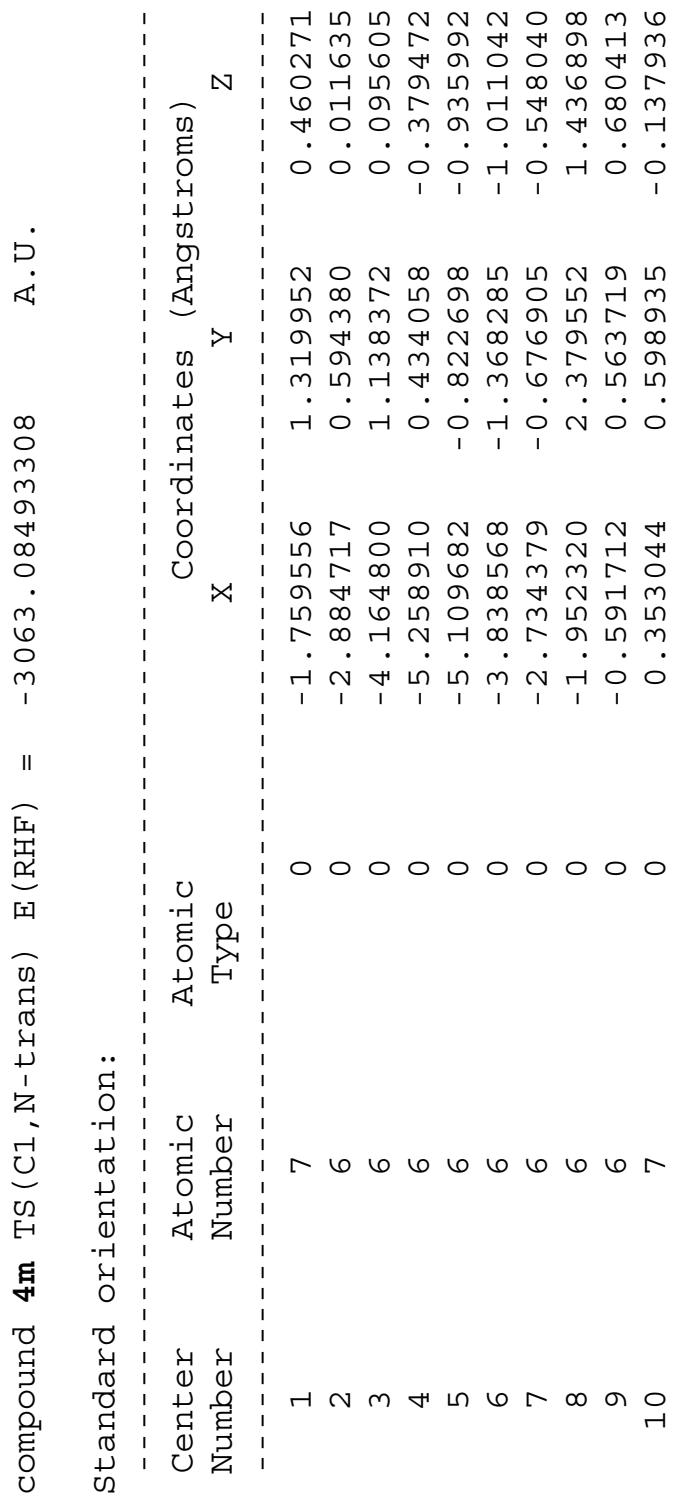




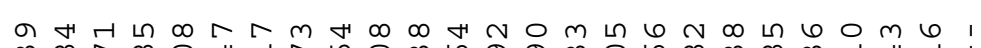

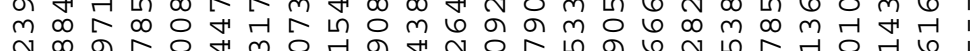

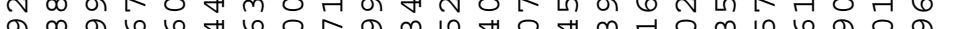

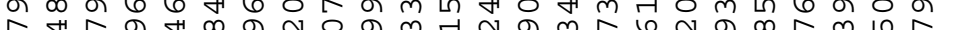

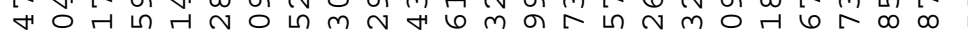

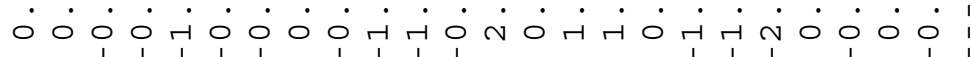

Nล स 1 न

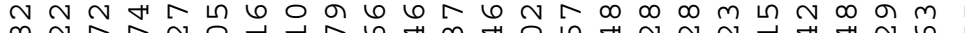

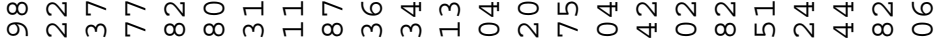

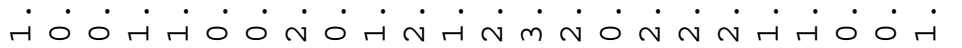

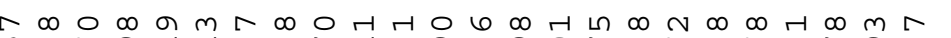

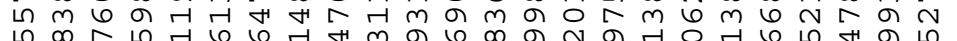

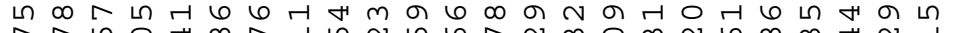
๙ v

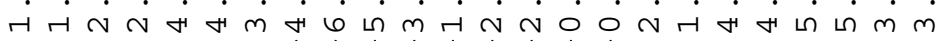
000000000000000000000000 4.

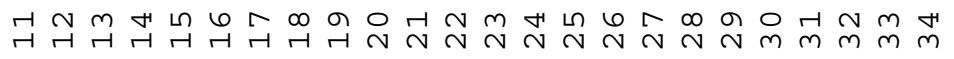

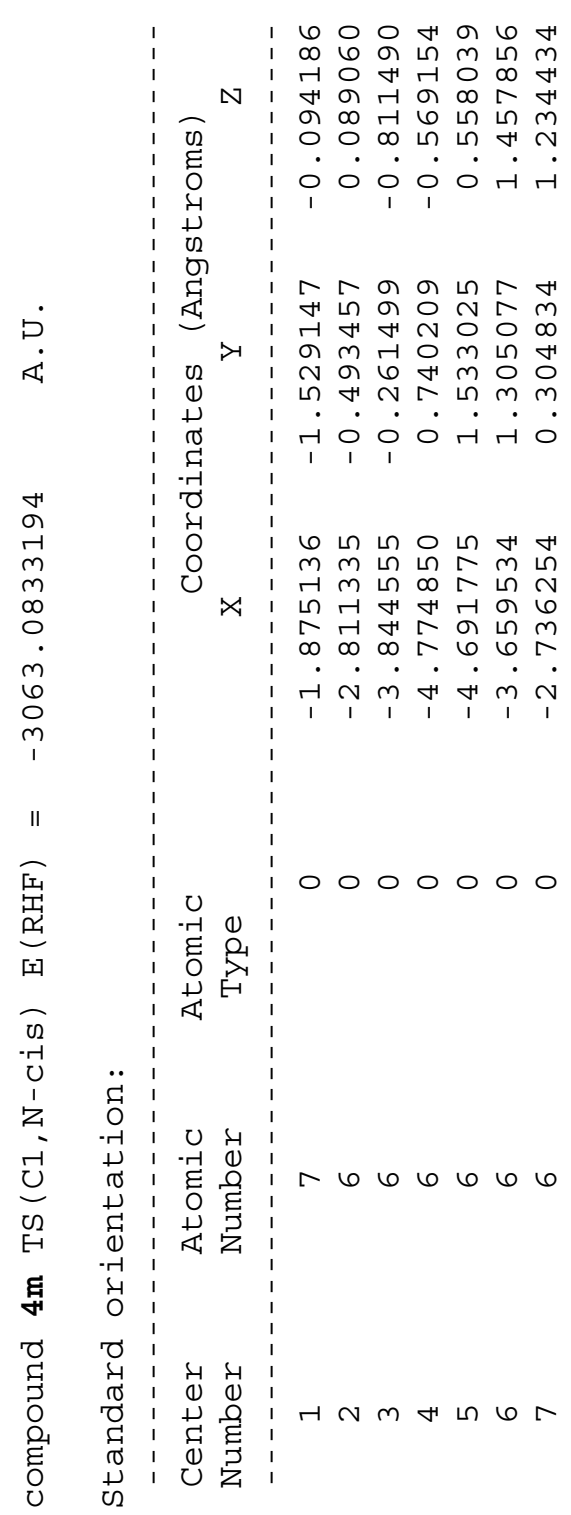




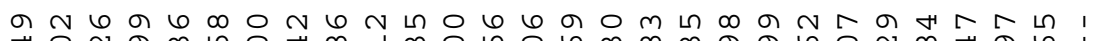

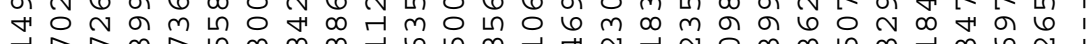

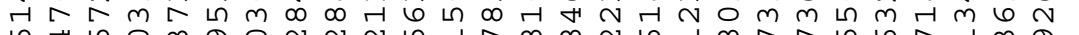
당

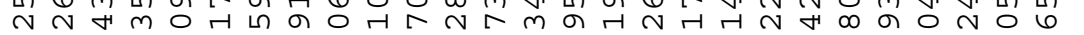

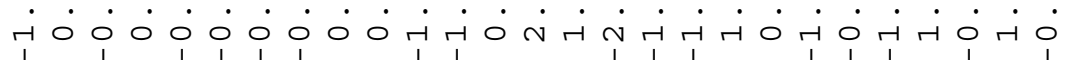

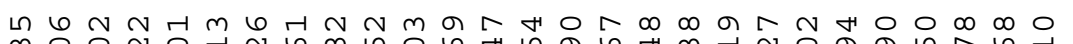

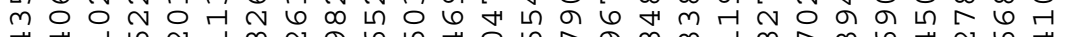
अ

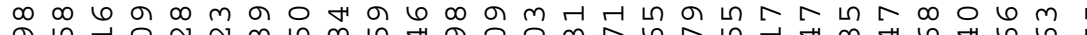
ब 1 म $m \sim$ ก $\mathrm{m}$ の

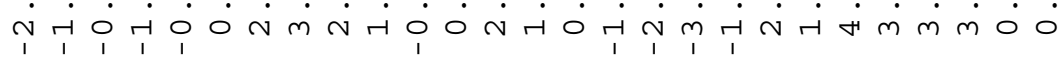

$\infty \Lambda$ の

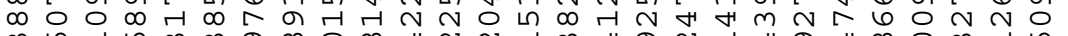
$m$ ம

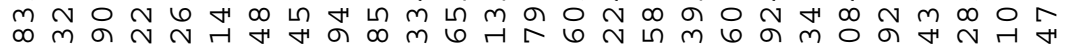

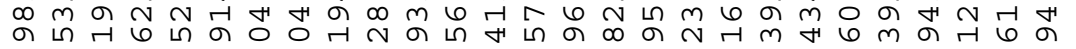

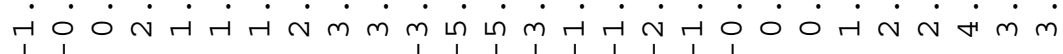

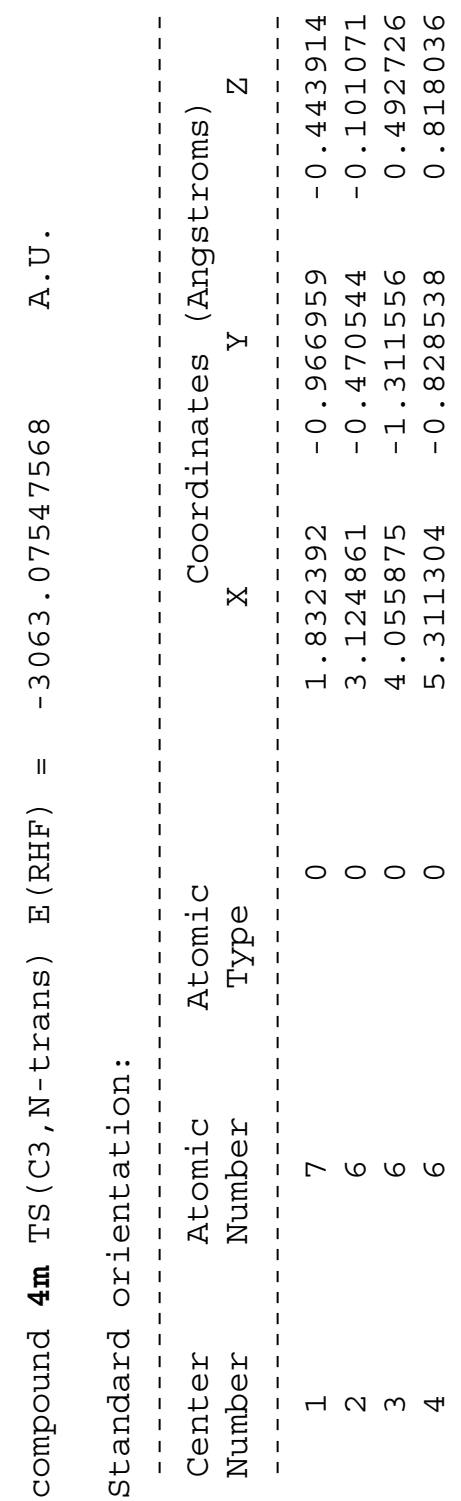




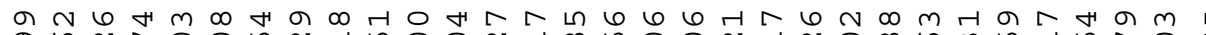

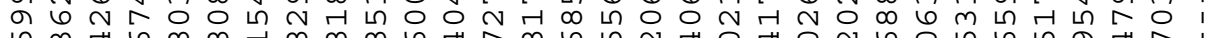

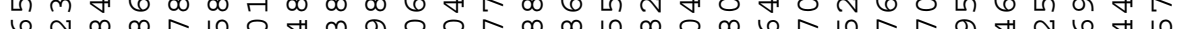

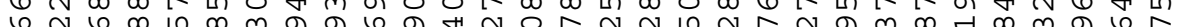

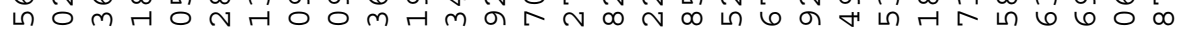

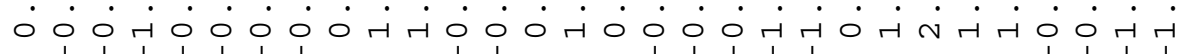

H

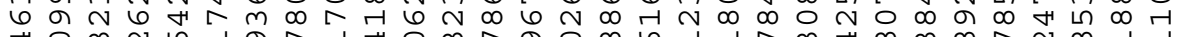
म० $m N$ L

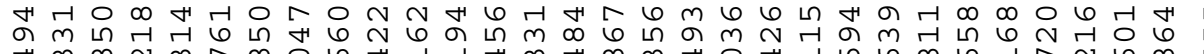

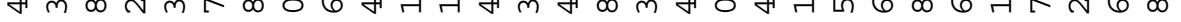

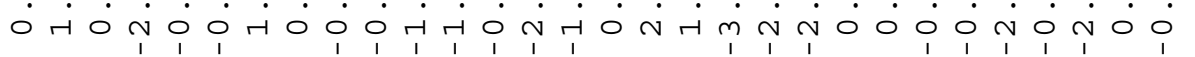

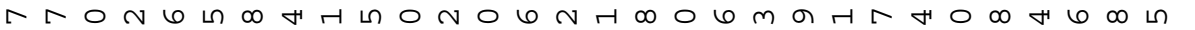
$\kappa$ เ

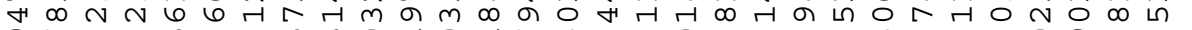

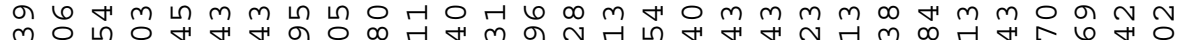
⿹유맘

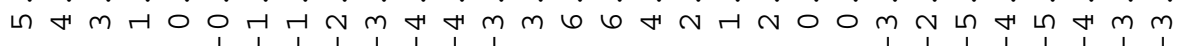

000000000000000000000000000000

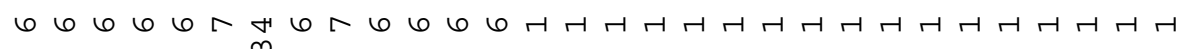

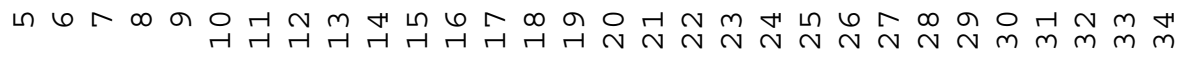




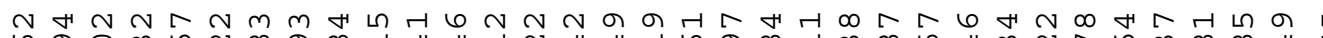

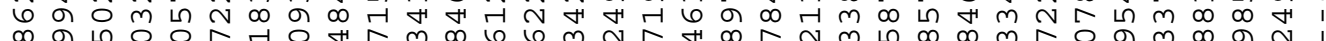
$\sigma$, ๙ बे H

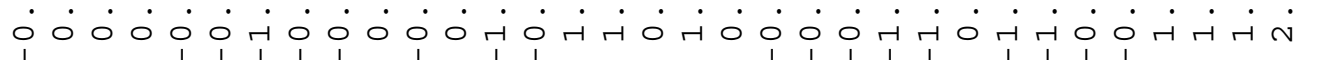

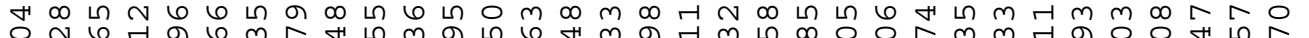

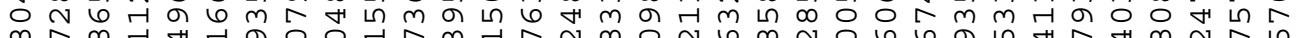

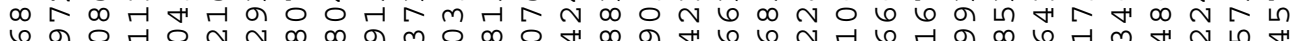

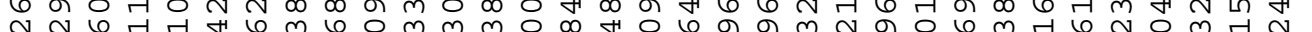
ग म 0 H

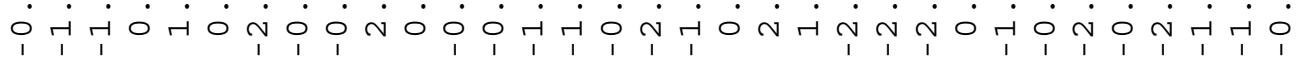

$6 m 0$ ก

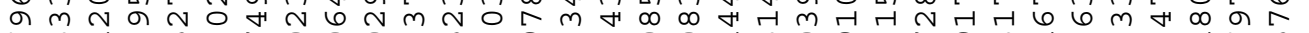

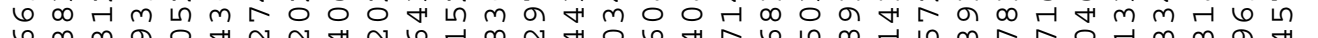

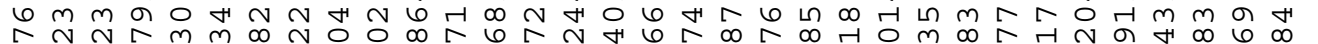

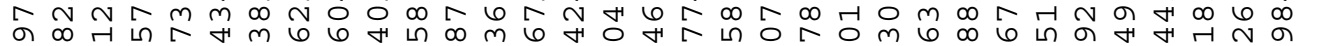

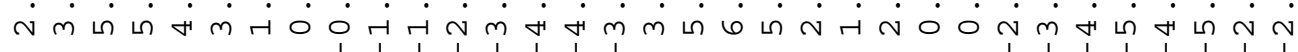

000000000000000000000000000000000

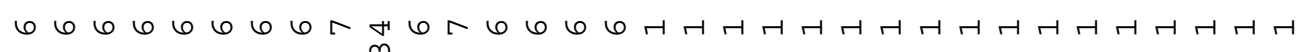

$\sim m$ म

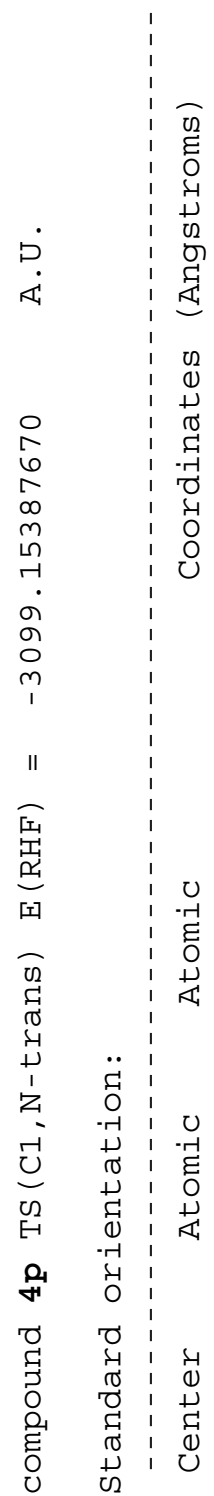




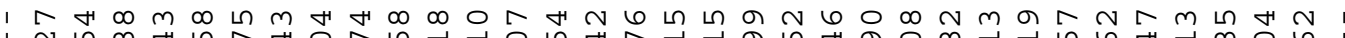
i n $\begin{aligned} & 1 \\ & 1\end{aligned}$

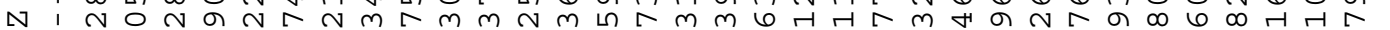

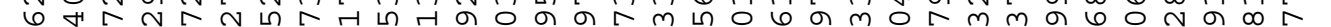

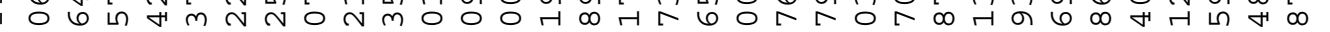

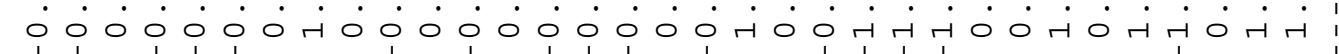

Hबの

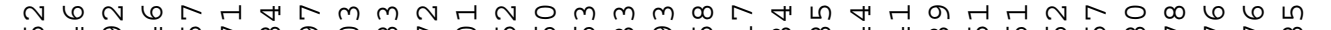
ก म

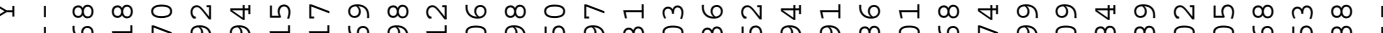
6 त N $A$ N N

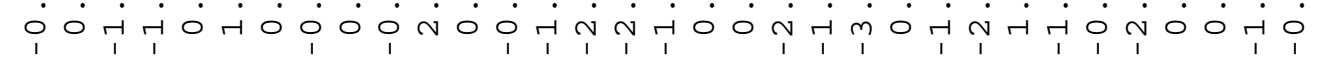

I 0 ○

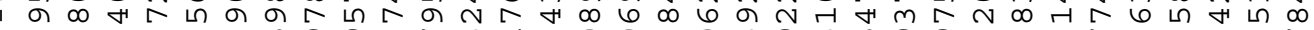

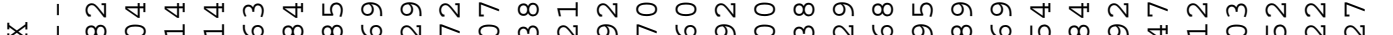

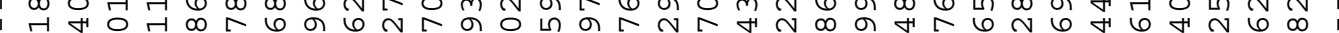

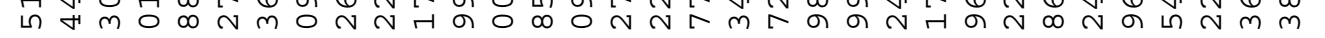

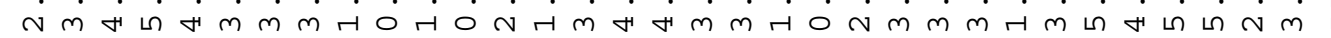

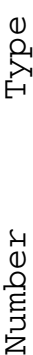




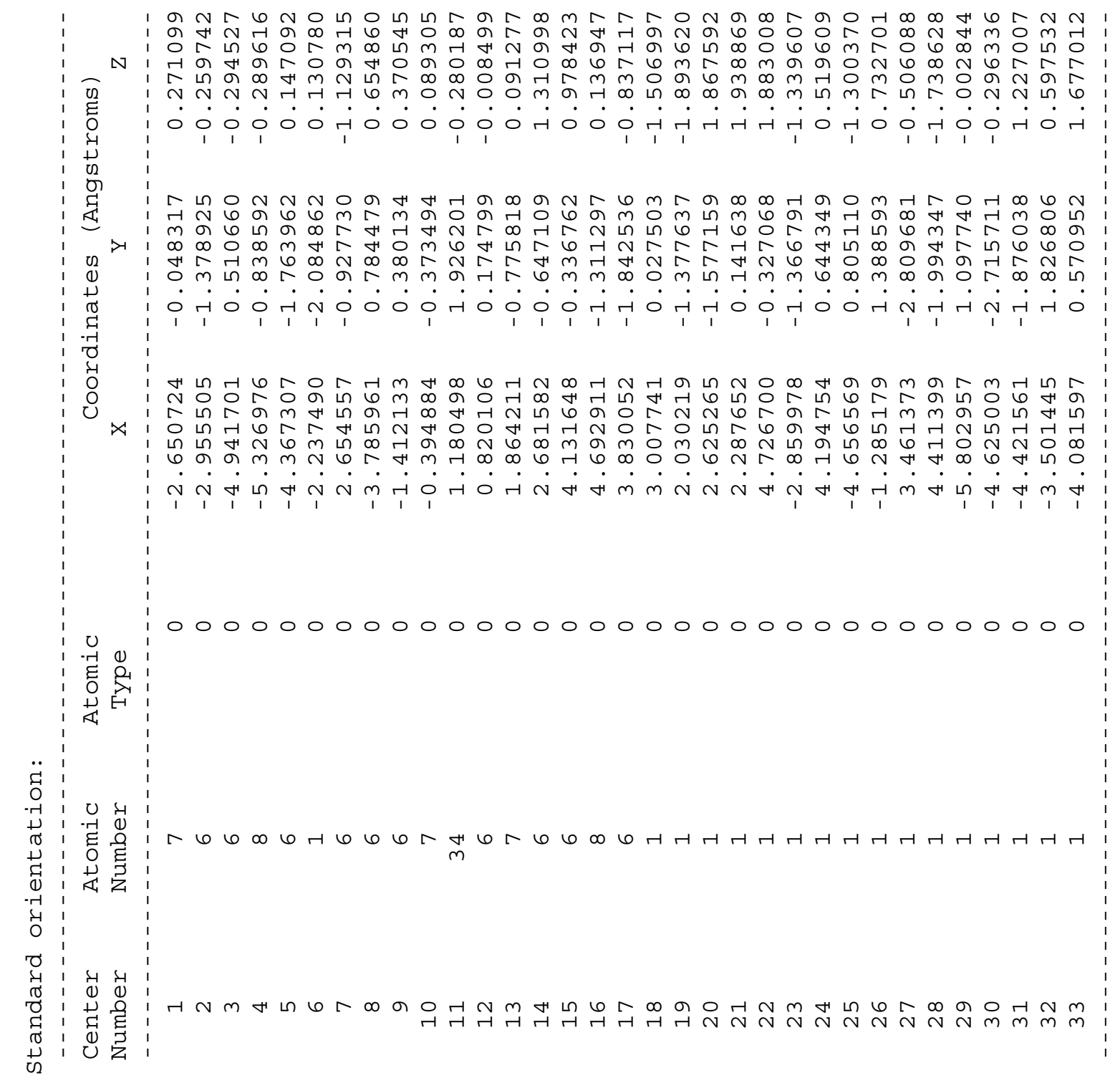




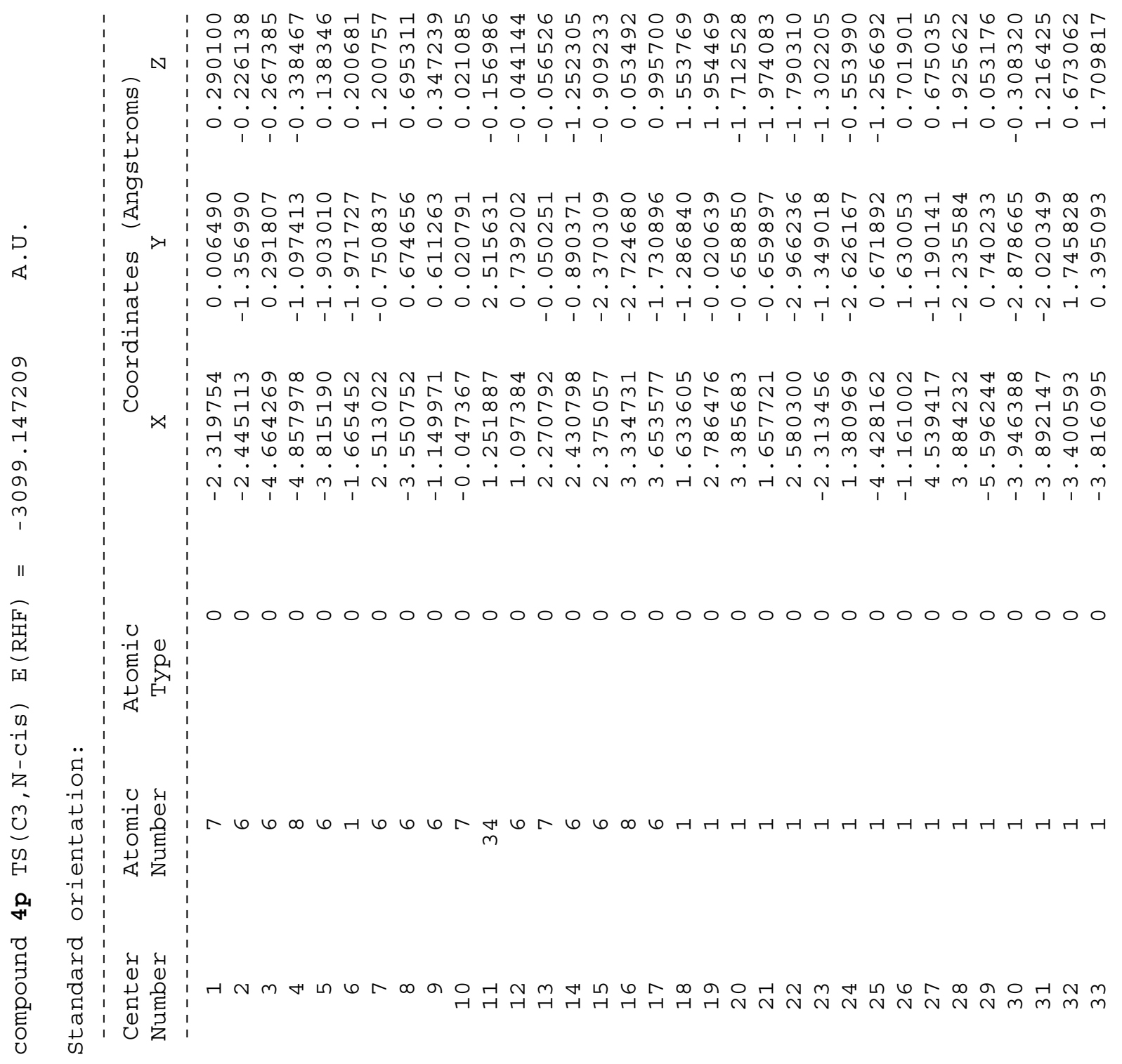


$\frac{n}{n}$ 